\title{
A circle of improvement in bleeding management : from laboratory to clinic and back
}

Citation for published version (APA):

Lance, M. D. (2013). A circle of improvement in bleeding management : from laboratory to clinic and back. [Doctoral Thesis, Maastricht University]. Maastricht University. https://doi.org/10.26481/dis.20130517ml

Document status and date:

Published: 01/01/2013

DOI:

$10.26481 /$ dis. $20130517 \mathrm{ml}$

Document Version:

Publisher's PDF, also known as Version of record

\section{Please check the document version of this publication:}

- A submitted manuscript is the version of the article upon submission and before peer-review. There can be important differences between the submitted version and the official published version of record.

People interested in the research are advised to contact the author for the final version of the publication, or visit the DOI to the publisher's website.

- The final author version and the galley proof are versions of the publication after peer review.

- The final published version features the final layout of the paper including the volume, issue and page numbers.

Link to publication

\footnotetext{
General rights rights.

- You may freely distribute the URL identifying the publication in the public portal. please follow below link for the End User Agreement:

www.umlib.nl/taverne-license

Take down policy

If you believe that this document breaches copyright please contact us at:

repository@maastrichtuniversity.nl

providing details and we will investigate your claim.
}

Copyright and moral rights for the publications made accessible in the public portal are retained by the authors and/or other copyright owners and it is a condition of accessing publications that users recognise and abide by the legal requirements associated with these

- Users may download and print one copy of any publication from the public portal for the purpose of private study or research.

- You may not further distribute the material or use it for any profit-making activity or commercial gain

If the publication is distributed under the terms of Article $25 \mathrm{fa}$ of the Dutch Copyright Act, indicated by the "Taverne" license above, 
A circle of improvement in bleeding management:

from laboratory to clinic and back.

Marcus D. Lancé 


\title{
A circle of improvement in bleeding management: from laboratory to clinic and back.
}

\author{
Proefschrift
}

ter verkrijging van de graad van doctor aan de Universiteit Maastricht op gezag van de Rector Magnificus, Prof. dr. L.L.G. Soete volgens het besluit van het College van Decanen, in het openbaar te verdedigen op

vrijdag 17 mei 2013 om 14.00 uur

door

Marcus D. Lancé

Geboren op 17 mei 1970 te Rott (Roetgen), Duitsland 


\section{Promotor}

Prof. Dr. M.A.E. Marcus

Prof. Dr. J.W.M. Heemskerk

\section{Copromotor}

Dr. Y.M.C. Henskens

\section{Beoordelingscommissie}

Prof. Dr. B. Mochtar, voorzitter

Prof. Dr. H. Crijns

Prof. Dr. Kruitwagen

Dr. B. De Laat

Prof. Dr. R. Rossaint (RWTH-Aken, Duitsland)

This thesis was kindly supported by CSL-Behring and TEM international Additional support for the publication of this thesis by Goodlife, Boehringer-Ingelheim, Orion-Pharma and Vifor Pharma 
"Everything is okay in the end, if it's not okay, then it's not the end"

Oscar Wilde

To my grandparents Karin \& Daniel 


\section{Contents}

Chapter 1 General introduction

Historical review over coagulation and measurement techniques

\section{Chapter 2}

a. Mean platelet volume as a diagnostic marker for cardiovascular disease: drawbacks of pre-analytical conditions and measuring techniques

b. Do we need time adjusted mean platelet volume measurements?

\section{Chapter 3}

Do blood collection methods influence whole-blood platelet function analysis?

\section{Chapter 4}

Influence of pneumatic systems:

a. The effects of pneumatic tube system transport on ROTEM analysis and contact activation assessed by thrombin generation test

b. Platelet concentrate transport in pneumatic tube systems - does it work?

\section{Chapter 5}

a. Obstetric hemorrhage and coagulation: an update

b. Platelet monitoring follow-up in a pregnant patient 109 with HELLP syndrome.

\section{Chapter 6}

a. Impaired thrombin generation and fibrin clot formation in patients with dilutional coagulopathy during major surgery.

b. Perioperative dilutional coagulopathy treated with fresh frozen plasma and fibrinogen concentrate: a prospective randomized intervention trial.

\section{Chapter 7}

$\begin{array}{ll}\text { General discussion } & 157\end{array}$

Summary 175

Samenvatting 181

Dankwoord 187

Curriculum Vitae 193

$\begin{array}{ll}\text { Publications } & 195\end{array}$ 
Chapter 1

General introduction 


\section{General introduction}

Historical review over coagulation and measurement techniques

Blood is the only fluid organ. However, it can clot to a solid mass under certain physiological (haemostasis) or pathological (thrombosis) conditions. Already the Greek Aristoteles (322 A.D.) reasoned that the clotting process is initiated by the outflow of blood from the body. An early idea was that the high body temperature is needed to keep blood in the fluid state, and this perception persisted until the $18^{\text {th }}$ century. At that time, also another concept on blood coagulation became popular, namely that blood was fluid only in a living individual but started to clot once outside the body in a "dead" environment [1]. However, with the $19^{\text {th }}$ and $20^{\text {th }}$ century knowledge of biochemical reactions [2], the process of blood coagulation is understood as an enzymatic cascade of proteolytic events, in which thrombin and platelets plays a central role in achieving fibrin formation and polymerization into a macroscopic clot.

In current clinical practice, it is important to dispose of laboratory tests that support rapid decision-making in diagnosis or treatment of bleeding, especially in the acute perioperative clinical setting. Adequate tests are needed for the coagulation process itself, for platelet function as well as for other important hemostatic components such as von Willebrand factor. For optimal diagnostic performance of these laboratory tests, not only the pre-analytical, analytical and post-analytical variables of blood samples need to be well controlled, but also the tests need to be carried out with sufficiently high sensitivity and specificity. Furthermore, especially for the point-of-care situation, test results need to be available as rapidly as possible with minimal blood sample preparation. Hence, there is great interest in whole-blood screening tests. A good screening test can be described as giving minimal discomfort to the patient, inexpensive, easy to perform, reliable with low (pre)analytical variability and discriminative.

In the present thesis we have used three different types of hemostatic screening tests (with plasma or whole blood) to predict hemostatic insufficiencies and to monitor the risk of acquired bleeding: 
1. Screening tests for plasmatic coagulation kinetics (prothrombin time, activated partial thromboplastin time, thrombin generation);

2. Screening tests for platelet function in plasma or blood (platelet function analyzer, multiple electrode impedance aggregometry);

3. Screening tests for coagulation capacity of whole-blood (thrombelastography, thromboelastometry).

Below, we briefly introduce these tests from a historical perspective.

\section{Screening for defects in plasma coagulation kinetics}

The first laboratory tests to measure blood coagulation parameters were developed in the $19^{\text {th }}$ century. An early method to assess the clotting time of blood was that of Vierordt [3], who used a white horse hair to draw through a glass tube containing freshly isolated blood, and determined the time that the hair became reddish (adherent red cells) or covered with coagulum (fibrin). The first description of $\mathrm{Ca}^{2+}$ scavenging reagents like oxalate to suppress the natural clotting of the blood was in the same period [4]. The use of anticoagulated blood samples allowed development of the first kinetic-based coagulation tests by the measuring the time interval from recalcification to initial fibrin clot formation. Citrate-anticoagulated plasma was used in the clotting time tests of Howell and Lee-White [5-7]. A major disadvantage of these was that they were global and did not differentiate between different types of coagulation abnormalities. Biochemical insight into the process of blood coagulation developed in the early $20^{\text {th }}$ century, when Morawitz presented a scheme in which prothrombin by $\mathrm{CaCl}_{2}$ activation yielded thrombin that converted fibrinogen into fibrin $[8,9]$. At present, assessment of fibrin formation using citrate-anticoagulated plasma and recalcification with $\mathrm{CaCl}_{2}$ is still the common procedure. Also the insight that coagulation measurement needs a kinetic approach - e.g. determining the time and rate of fibrin clot formation - has remained unchanged.

Prothrombin time (PT). In 1935, Quick developed the first prothrombin time (PT) assay for measurement of fibrin clot formation in platelet-poor plasma (PRP). In the literature, this is still referred to as the Quick time. This method was modified by Owren [10-12]. Later in 1954 brain thromboplastin (a preparation containing human tissue factor and phospholipids) was added to stabilize the test results. This modified 
PT test rapidly became the standard one-stage coagulation test in clinical laboratories [13]. Since plasma is triggered here with a source of tissue factor, phospholipids and $\mathrm{CaCl}_{2}$, the PT monitors the so-called extrinsic pathway of the coagulation process.

The discovery of vitamin $\mathrm{K}$ as a key nutritional component controlling coagulation came from studies to the origin of sweet clover disease in cow and sheep, which presented in these animals as a bleeding tendency after intake of large amounts of sweet clover [14]. The cause of bleeding appeared to be the substance dicoumarol, which acts as a vitamin $\mathrm{K}$ antagonist, blocking the formation of active vitamin K-dependent coagulation factors [15]. Already in 1942, dicoumarol was clinically introduced as vitamin $\mathrm{K}$ antagonist in the treatment of thromboembolism due to atrial fibrillation, while the Quick time assay was used to assess the treatment efficacy [16, 17]. It soon appeared that monitoring of the PT in seconds was imprecise, because of the variability between thromboplastin preparations. Commercial thromboplastins can be derived from either human or animal tissues, or be reconstituted from purified components (recombinant tissue factor and synthetic phospholipids). To reduce inter-assay variability and come to a standardization of the PT test, the international normalised ratio (INR) was introduced in 1983 [18]. This is the ratio of the patient's plasma prothrombin time and the prothrombin time of a standardized normal pool plasma, which is measured by the use of a WHO reference thromboplastin sample.

Currently, the standardized INR is used worldwide to check the anticoagulant state of patients on vitamin $\mathrm{K}$ antagonists. The PT is still a valid screening test for congenital (hemophilia) or acquired (liver disease, disseminated intravascular coagulation) abnormalities, although its sensitivity and specificity are dependent on the activating reagent and the analytical equipment.

Activated partial thromboplastin time (aPTT). The inability to diagnose haemophilias with the Quick test stimulated Brinkhous and colleagues to develop a more feasible test, the partial thromboplastin time [19]. The name partial thromboplastin time was chosen, because coagulation in plasma was only triggered with $\mathrm{CaCl}_{2}$ and phospholipids, leaving out tissue factor. Because the method was laborious and unstable, Rapaport and colleagues reported in 1961 a stabilised version by adding kaolin (a clay mineral), which acts as activator of the intrinsic 
pathway of coagulation [20,21]. The result was the activated partial thromboplastin time (aPTT) test. This test is widely used in clinical practice, even though the commercially available tests can differ in type of activating reagent (kaolin, ellagic acid, silica) and in general assay performance [22, 23].

Because of its global nature, the aPTT is of limited sensitivity to coagulation factor deficiencies or anticoagulant therapies. Individual coagulation factor deficiencies in plasma need to be $>70 \%$ to give a prolonged test result. Multiple factor deficiencies may cause prolongation when being less severe. A prolonged test outcome is also obtained with substances interfering in the coagulation process, fibrin degradation products, and anticoagulants like heparin. The APTT is less sensitive for vitamin $\mathrm{K}$ deficiency and oral vitamin $\mathrm{K}$ antagonists compared to the PT. Unfortunately, it is also sensitive to sample collection artefacts, which may result in artificial prolongation.

In daily practice, the aPTT is used to screen for intrinsic factor deficiencies and to monitor for heparin levels [24]. National and international guidelines on the management of massive bleeding in surgery and trauma still recommend the aPTT as screening test to guide hemotherapy [25-27]. However, they also mention shortcomings of the test, such as a high turn-around time and information on only the initial stage of the coagulation process. Guidelines to reduce the pre-analytical and analytical variables in the measurement of PT, INR and aPTT have been published for the Netherlands [28]. Yet, there is a need for better tests to monitor the whole coagulation process.

Thrombin generation test. There is revived interest in the measurement of thrombin generation, which mostly occurs as a global assay determining the integrated 'working power' or capacity of thrombin in plasma, which is triggered with specific activators (e.g., tissue factor or kaolin). This contrasts to the PT and aPTT, which monitor the initial formation of a fibrin clot, at a time point when only $4 \%$ of the total amount of thrombin is generated. Hence, the thrombin generation test may give more information on the later stages of the coagulation process.

The first thrombin generation assays, published in 1953, were laborious and technically difficult to perform, as they required sub-sampling from a plasma mixture at regular intervals into series of tubes containing fibrinogen, in which then clotting times were determined $[29,30]$. The latter were used to estimate the thrombin 
concentration at the time of sampling, by calibration with a dose range of thrombin standard. In 1986, the Hemker group replaced the fibrinogen tubes by vials with a thrombin-cleavable chromogenic substrate, in order to obtain time-plots of the generation and subsequent inactivation of thrombin in plasma, which allowed calculation of the area-under-the-thrombin curve [31]. The endogenous thrombin potential was introduced as a parameter based on the area-under-the-curve, and thus reflecting the total activity of thrombin formed during the course of the test. However, it soon appeared that the curves needed a correction. In plasma thrombin is rapidly inactivated by both antithrombin and $\alpha 2$-macroglobulin. Whilst the thrombin bound to antithrombin is unable to cleave chromogenic substrate, the thrombin bound to a2-macroglobulin retains its chromogenic activity, but it is no longer active in the coagulation process. Hence, an algorithm was employed to correct for the (non-physiological) contribution of thrombin bound to a2-macroglobulin to the substrate cleavage. One limitation was that the early versions of the thrombin generation test required prior defibrination of the plasma to prevent quenching of the chromophore by fibrin clots.

Later, replacement of the chromogenic substrate with a low-affinity fluorogenic substrate enabled not only the testing of plasma (or PRP) with fibrinogen, but also the continuous measurement of fluorescence development in one sample without the need of sub-sampling. Another modification was the simultaneous measurement of a calibration curve, consisting of the fluorescence development of a constant amount of thrombin in the same plasma. This led to the so-called calibrated automated thrombogram (CAT) assay [31]. Alternative methods have been developed as well. Current thrombin generation assays have been used to detect coagulation hypo- and hyper-function as well as the role of platelets in coagulation [32-36]. However, the method of triggering and other test circumstances can change from study to study. This requires caution when comparing data from different studies and when applying the results to therapeutic strategies [36]. Furthermore, variation in the pre-analytical conditions of sample preparation can influence the test outcome [37]. Another limitation is the need to prepare (platelet-rich) plasma from blood samples, which makes it not suitable as a bedside test. This has recently stimulated the Hemker laboratory to develop a whole-blood thrombin generation test [38], which is currently validated and standardized. 


\section{Screening for platelet function in plasma or whole blood}

The first platelet function test was published in 1910, when Duke described a method to measure the bleeding time in healthy volunteers. With a small lancet a wound was made in the forearm, and the time was measured until stopping of the bleeding [39]. Modified versions of this test, used in current clinical practice, are still in use and can inform on the process of primary hemostasis in vivo, particularly reflecting the prohemostatic activities of platelets and von Willebrand factor. A drawback of bleeding time measurements is that these are cumbersome and difficult to standardize [40].

The first test to check for platelet function in vitro, described in 1941, monitored the adhesion of platelets to a glass surface, which reflected the platelet count in blood [41]. Later in 1962, Born described the meanwhile classical method of platelet aggregation measurement by light transmission aggregometry (LTA). In the original assay, platelets in plasma were stimulated with ADP and the increase in light transmission of the initially turbid PRP sample was determined [42]. Currently, LTA is routinely applied with many other platelet-activating substances at a range of concentrations [e.g., ADP, epinephrine, arachidonic acid, thrombin receptoractivating peptide (TRAP), collagen, thromboxane analogue]. The LTA is considered as golden standard of platelet function testing. Thrombin as a potent platelet agonist was firstly used by Zucker and colleagues [43, 44]. Limitations of platelet function analysis by LTA are that the test is laborious, since it requires the preparation of $\mathrm{PRP}$, that it is subjected to inter-laboratory variation and requires skilled personnel $[45,46]$. Current guidelines and references for LTA still differ in pre-analytical and analytical variables [47-49]. Furthermore, tests based on LTA do not take into account the interactions between platelets and other blood cells.

Multiple electrode impedance aggregometry in whole blood. In 1980 the technique of multiple electrode impedance aggregometry (MEIA) was introduced, which allows platelet aggregation studies in whole blood to measure agonist-induced platelet aggregation in a way sensitive to indomethacin or aspirin [50]. The impedance aggregometry method detects the changes in electrical conductance due to adhesion of platelet aggregates to an electrode placed in a stirred whole blood or a PRP sample. The initial electrodes consisted of platinum and were not disposable, 
meaning that time was needed for their cleaning. An important move forward was the commercial availability of whole-blood Chronolog impedance aggregometer, which dispose of non-disposable palladium electrodes and have been used for several studies [51, 52]. A next generation machine, also using whole-blood, is provided by the MEIA test. In a multi-tube-based assay, a range of electrodes is placed in disposable cups, which are stirred with a small magnet [53]. The electrodes record platelet deposition and aggregation in each cup, and hence give immediate information on platelet function. This system is attractive for point-of-care use. The commercially available device is commonly used to test for up to five platelet agonists (ADP, arachidonic acid, TRAP, collagen or ristocetin). Current standardisation issues in MEA concern the way of blood drawing, the type of anticoagulant in the test tube, and the minimally required platelet count and hematocrit [54].

Platelet function analyzer. The Platelet function analyzer-100 (PFA-100) was introduced in 1995 as a method to assess platelet function in citrated whole blood under shear stress $[55,56]$. In this assay, anticoagulated whole blood is injected under constant flow into a capillary tube, containing a grid covered with collagen and epinephrine or ADP. The time needed to occlude the grid by aggregated platelets (closure time) is measured as an indicator of platelet function. The capillary is incorporated in a disposable cartridge, which makes the device robust and easy to use. Although the first studies suggested that the closure time measured with the PFA-100 can be used as a proxy for the in vivo bleeding time, later more thorough investigations showed that its sensitivity is limited, and main confined to the detection of von Willebrand disease, severe platelet disorders and, in most cases, the efficacy of patient treatment with aspirin [57]. Moderate or mild thrombocytopathies however are not detected with the PFA-100 [58].

\section{Screening for coagulation capacity in whole blood}

There is increasing interest in the use of tests to determine the overall coagulation potential or capacity in whole blood samples. The newly developed method of wholeblood thrombin generation (see above) also falls in this category. 
Thrombelastography was introduced in 1948 as a method to follow the kinetics of the complete coagulation process in whole blood [59]. In the original method fresh whole blood in a cup is mixed with a coagulation activator like kaolin. A pin, connected to a torsion wire, is brought into the mixing cup and the cup is continuously turned to an angle of 4.45'. The gradual formation of a fibrin clot increases the torsion strength and damps the movement of the pin until, at complete fibrin formation, the pin becomes almost fixed. Registration of the movement of the pin in time gives characteristic graphs, from which the start of fibrin formation, and the time and maximal formation of elastic fibrin clots can be read. The method also detects gradual resolution of the clot due to fibrinolysis [59]. Soon after its description, the thromboelastography method was criticized, because its high sensitivity to artificial vibrations and the lack of detecting single factor deficiencies [5, $60]$. However, it was tested with success to test prophylactic anticoagulation as well as heparin or anti-fibrinolytic therapy [61-63]. In the mid 1980ties, it was picked up as a possible bedside point-of-care test for monitoring hemostasis after liver transplantation [64] or major cardiac surgery [65].

In the last decade, after introducing small modifications and automation of the technique, thrombelastography has become a method with a wider range of applications [66]. Currently, two (semi)automated commercial devices are on the market. The thromboelastometry (TEM) apparatus uses a fixed cup with a pin that is rotating [67]. The other thrombelastography (TEG) system makes use of the classical method with rotating cup [68]. While being designed as kinetic tests, both methods effectively measure the capacity of the coagulation process in terms of maximal fibrin clot formation [68]. During the last 5 years, both commercial systems have been shown to be suitable for detecting and treating coagulopathy during massive perioperative bleeding, especially in patients were fibrinogen levels become low [6971]. It is expected that thromboelastography will become an important method in guiding haemostatic therapy at the bedside, particularly in the operation theatre. This point of care device may also predict bleeding during obstetric surgery, at the emergency department, and at the intensive care unit [72, 73]. 


\section{Which are the best tests to use perioperatively?}

Conventional tests of clotting times, such as PT, INR and aPTT, are insufficiently sensitive to detect bleeding risks in patients before, during and after surgery. Potentially more useful are the newer capacitative coagulation tests measuring thrombin generation (CAT) and elastic fibrin clot formation (thromboelastography). However, these tests still require further standardization with regard to pre-analytical variables and cutoff values for specific clinical settings. The clot formation tests (TEM, TEG) are already being used as bedside point-of-care tests, thus linking the hospital laboratory directly into the clinic (operation theatre, intensive care unit, heart catheter laboratory). Current whole-blood platelet function tests (MEIA, PFA-100) are also being brought to the bedside.

It is foreseen that, collectively, these whole-blood assays offer a more comprehensive insight into the hemostatic process in acquired, perioperative bleeding or trauma than the PT or aPTT. The advantages are obvious. Test results are available within minutes, and they provide immediate information on various parts of the hemostatic system, coagulation initiation and capacity and platelet count and function. Ideally, these tests can guide the prohemostatic therapy according to the actual needs of each patient during and after the intervention, thus avoiding any over- or under-transfusion. The beneficial effects of whole-blood bedside tests have indeed been discussed in the literature with respect to transfusion treatment of bleeding patients, or drug treatment of thrombosis patients who are resistant to platelet inhibitors $[68,71,74,75]$ in trauma care, the bedside monitoring of the hemostatic process can guide transfusion of patients with blood products (reds cells, fresh-frozen plasma, platelet concentrates), also to reduce further hemodilution that may aggravate the bleeding [76, 77]. On the other hand, overtreatment with prohemostatic interventions may give rise to thrombotic complications [78]. An important discussion point is whether the point-of-care tests should be performed in the operating room or in the central hospital laboratory with dedicated and trained technicians under the supervision of a laboratory specialist [79-82].

The overall aim of this thesis is to come to a better performance of the circle of quality improvement in bleeding management (Figure 1). This quality improvement relies on close collaboration between care givers and the hospital laboratory in order to continuously ameliorate the treatment of hemorrhagic patients. Therefore, 
investigations were carried out to determine how laboratory tests can improve diagnostics in hemostatic control of a given patient, and can help to develop a patient-tailored therapy plan stopping bleeding earlier.

\section{Outline of this thesis}

Following chapter 1, the present introduction, chapter 2 illustrates the importance of precise pre-analytical conditions for the measurement of mean platelet volume, which may be considered as a surrogate parameter for platelet activity. A review describes the potential drawbacks in using and implementing this haemostasis parameter. In addition, a study is presented to perform standardized measurements of mean platelet volume with reference ranges. Described in chapter $\mathbf{3}$ are key pre-analytical factors influencing platelet function analysis using PFA-100 and whole-blood aggregometry. Chapter 4 focuses on a practical clinical question, how to transport blood samples and products in the hospital, particularly for the point-of-care testing of patients undergoing surgery. In chapter $\mathbf{4 a}$, the reliability is examined of sending blood samples via a pneumatic system between the operation theatre and the laboratory for thromboelastometric analysis. Subsequently, in chapter 4b the feasibility of transport of platelet concentrates through the pneumatic system is investigated.

Chapter 5a gives a comprehensive description of current treatment options for obstetric patients suffering from peripartal bleeding. The review focuses on the potential improvement of treatment directed by point-of-care devices measuring coagulant activity. Chapter 5b describes how modern diagnostic tools can help in predicting the bleeding risk in an obstetric patient. In chapter $\mathbf{6 a}$ the effects of haemorrhage and hemodilution on capacitative coagulation parameters (thrombin generation and fibrin clot formation) are investigated in three groups of patients. In the ensuing chapter $\mathbf{6 b}$, a new treatment strategy (infusion of fibrinogen concentrate) was prospectively studied in patients suffering from massive haemorrhage due to major elective surgery. The results from the various chapters are put together in chapter 7, where a 'representative' patient is presented, who may benefit from improvements of point-of-care diagnostics by close collaboration of the clinicians and laboratory specialists, with also attention to future developments. 


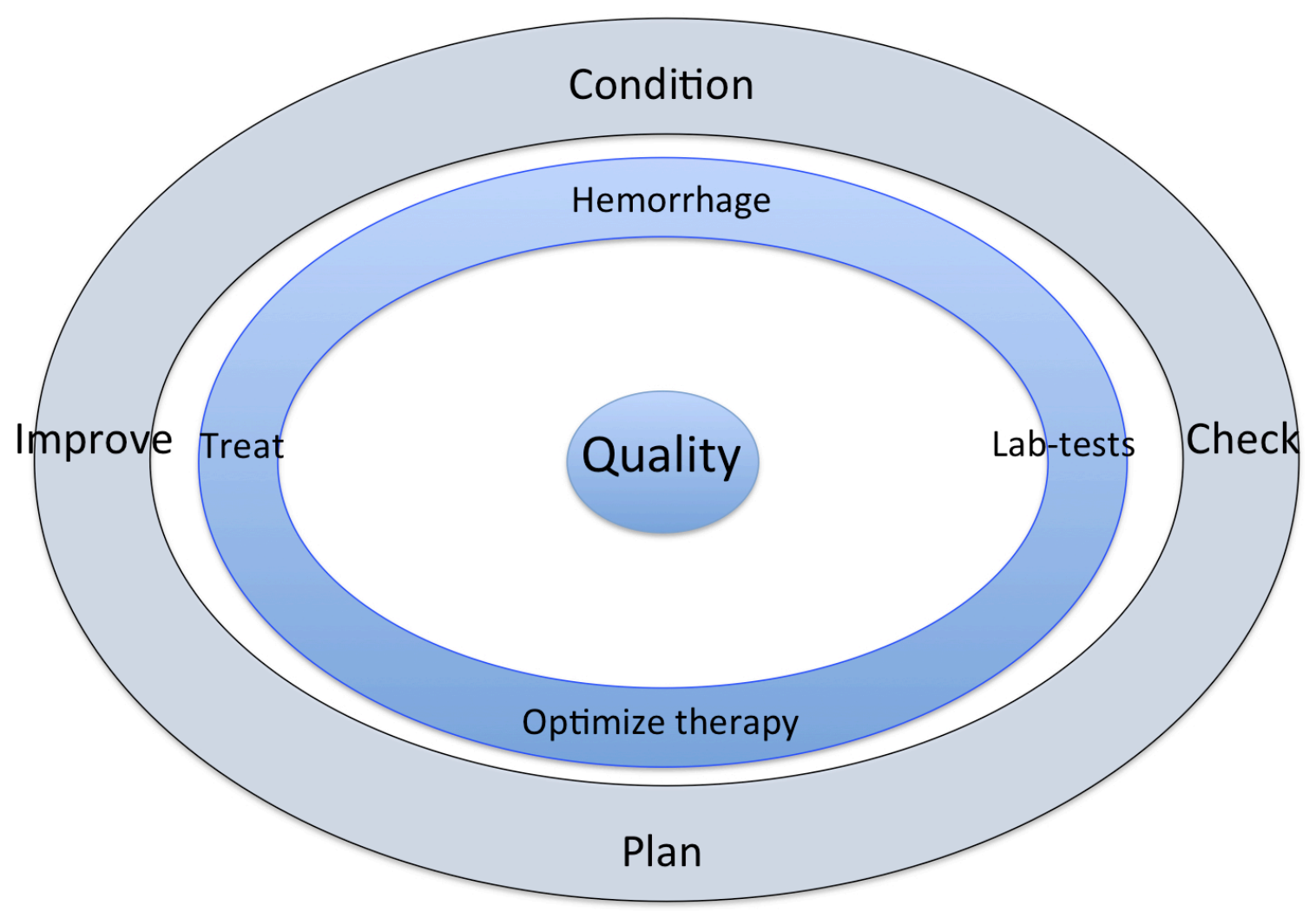

Fig. 1: A quality circle of improvement in bleeding management. Starting with hemorrhage in a given patient, laboratory tests are needed to improve diagnostics and come to an optimized therapy plan. The chosen treatment of the patient should stop the bleeding. However, in trauma and surgery the circle needs to be repeated at the next bleeding situation, where the process of quality improvement starts again. 


\section{References}

$1 \quad$ Bauer AW, Mall K: Historische Konzepte zur Physiologie und Pathologie der Blutgerinnung. Haemostaseologie. 1995;15: 92-9.

2 Hemker CW: De ontdekking van het prothrombine. Amsterdam: Meesters, Amsterdam; 1981.

3 Vierordt $\mathrm{H}$ : Die Gerinnungszeit des Blutes in gesunden und kranken Zuständen. Arch $f$ (physiologische) Heilkunde. 1878;19: 193-9.

4 Arthus NM, Pagès C: Nouvelle théorie chimique de la coagulation du sang. Archives de physiologie normale et pathologique. 1890;5: 739-46.

Strassner H: Die Entwicklung der Thromboelastographie ans Messverfahren; Med Diss der technischen Universität Muenchen. München, 2002. Howell WH: Hemophilia: the Wesley M. Carpenter lecture. Bull NY Acad Med.; 1939. Hougie C: The Lee and White test for coagulation time. Am J Clin Path. 1961;36: 537-8.

Morawitz P: Die Chemie der Blutgerinnung. Ergebnisse der Physiologie. 1905;4: 307-422.

Boulton F: A hundred years of cascading started by Paul Morawitz (1879-1936), a pioneer of haemostasis and of transfusion. Transfus Med. 2006;16: 1-10.

10 Quick AJ: The prothrombin time in hemophilia and in obstructive jaundice. J Biol Chem. 1935;109: 73-4.

11 Owren PA: The coagulation of blood. Investigations on a new clotting factor. Acta Med Scand. 1947;194: 521-49.

12 Owren PA: The activator complex of prothrombin and its clinical importance. Revue d'Hematologie. 1951;6: 135-47.

13 Bell WN, Alton HG: A brain extract as a substitute for platelet suspensions in the thromboplastin generation test. Nature. 1954;174: 880-1.

14 Dam H: The antihaemorrhagic vitamin of the chick. Biochem J. 1935;29: 1273-85.

15 Campbell HA, Link KP: Nutrition classics from The Journal of Biological Chemistry 138:21-33, 1941. Studies on the hemorrhagic sweet clover disease. IV. The isolation and crystallization of the hemorrhagic agent by Harold A. Campbell and Karl Paul Link. Nutr Rev. 1974;32: 244-6. Shapiro S, Redish MH, Campbell HA: Prothrombin studies. III. Effect of vitamin K upon hypopothrombinemia induced by dicumarol in man. Proc Soc Exper Biol \& Med. 1943;52: 125.

Quick AJ: Experimentally induced changes in the prothrombin level of the blood; quantitative studies in dogs given dicumarol; the effect of methylxanthines on prothrombin per se and when administered with dicumarol. J Biol Chem. 1945;161: 33-44.

Poller L: International normalized ratios (INR): the first 20 years. J Thromb Haemost. 2004;2: 849-60.

Wagner RH, Roberts HR, Webster WP, Shanbrom E, Brinkhous KM: Glycine-precipitated antihemophilic factor concentrates and their clinical use. Thromb Diathes Haemorrh Suppl. 1968;35: 41-8.

defects. J Clin Pathol. 1958;11: 406-9.

Margolis $\mathrm{J}$ : Activation of a permeability factor in plasma by contact with glass. Nature. 1958;181: 635-6.

22 Rapaport SI, Vermylen J, Hoylaerts M, Saito H, Hirsh J, Bates S, Dahlback B, Poller L: The multiple faces of the partial thromboplastin time aPTT. J Thromb Haemost. 2004;2: 2250-9. White GC, 2nd: The partial thromboplastin time: defining an era in coagulation. J Thromb Haemost. 2003;1: 2267-70.

24 McCraw A, Hillarp A, Echenagucia M: Considerations in the laboratory assessment of haemostasis. Haemophilia. 2010;16 Suppl 5: 74-8.
Centraal
Begeleidings
Orgaan
$(\mathrm{CBO}),:$
Richtlijn
bloedtransfusie. http://wwwcbonl/thema/Richtlijnen/Overzicht-richtlijnen/?p=519. 2011.

26 National Blood Authorities Australia (NBA): Patient blood management guidelines.. http://wwwnbagovau/guidelines/module1/cbmtpdf. 2011.

27 Rossaint R, Bouillon B, Cerny V, Coats TJ, Duranteau J, Fernandez-Mondejar E, Hunt BJ, Komadina R, Nardi G, Neugebauer E, Ozier Y, Riddez L, Schultz A, Stahel PF, Vincent JL, Spahn DR: Management of bleeding following major trauma: an updated European guideline. Crit Care. 2010;14: R52. Stichting Kwaliteitsbewaking Medische Laboratoriumdiagnostiek (SKML): Preanalytische voorschriften voor stollingsbepalingen. 2012 www.nvkc.nl/.../documents/Pre_Analytische_voorschriften_2012.pdf. 
Macfarlane RG, Biggs R: A thrombin generation test; the application in haemophilia and thrombocytopenia. J Clin Pathol. 1953;6: 3-8.

30 Pitney WR, Dacie JV: A simple method of studying the generation of thrombin in recalcified plasma; application in the investigation of haemophilia. J Clin Pathol. 1953;6: 9-14.

31 Hemker HC, Al Dieri R, De Smedt E, Beguin S: Thrombin generation, a function test of the haemostatic-thrombotic system. Thromb Haemost. 2006;96: 553-61.

Ten Cate-Hoek AJ, Dielis AW, Spronk HM, van Oerle R, Hamulyak K, Prins MH, ten Cate H: Thrombin generation in patients after acute deep-vein thrombosis. Thromb Haemost. 2008;100: 240-5.

33 Siegemund T, Petros S, Siegemund A, Scholz U, Engelmann L: Thrombin generation in severe haemophilia $A$ and $B$ : the endogenous thrombin potential in platelet-rich plasma. Thromb Haemost. 2003;90: 781-6.

34 Giroud M, Dutrillaux F, Lemesle M, Volot F, Lorenzini JL, Becker F, Dumas R: Coagulation abnormalities in lacunar and cortical ischemic stroke are quite different. Neurol Res. 1998;20: 15-8.

35 Haider AW, Andreotti F, Thompson GR, Kluft C, Maseri A, Davies GJ: Serum lipoprotein(a) level is related to thrombin generation and spontaneous intermittent coronary occlusion in patients with acute myocardial infarction. Circulation. 1996;94: 2072-6.

Wolberg AS: Thrombin generation assays: understanding how the method influences the results. Thromb Res. 2007;119: 663-5.

Loeffen R, Kleinegris MC, Loubele ST, Pluijmen PH, Fens D, van Oerle R, Ten Cate H, Spronk HM: Pre-analytical variables of thrombin generation: towards a standard procedure and validation of the method. J Thromb Haemost. 2012, in press.

Ninivaggi M, Apitz-Castro R, Dargaud Y, de Laat B, Hemker HC, Lindhout T: Whole-blood thrombin generation monitored with a calibrated automated thrombogram-based assay. Clin Chem. 2012;58: 1252-9.

Duke WW: The relation of blood platelets to hemorrhagic disease: description of a method for determining the bleeding time and coagulation time and report of three cases of hemorrhagic disease relieved by transfusion. J Am Med Assoc. 1910: 1185-92.

40 Harrison P: Assessment of platelet function in the laboratory. Hamostaseologie. 2009;29: 2531.

41 Payling-Wright H: Adhesiveness of blood platelets. J Path Bact. 1941;53: 255-58.

42 Born GV: Aggregation of blood platelets by adenosine diphosphate and its reversal. Nature. 1962;194: 927-9.

43 Zucker MB, Borrelli J: Relationship of some blood clotting factors to serotonin release from washed platelets. J Appl Physiol. 1955;7: 432-42.

44 Packham MA, Rand ML: Historical perspective on ADP-induced platelet activation. Purinergic Signal. 2011;7: 283-92.

45 Cattaneo M, Hayward CP, Moffat KA, Pugliano MT, Liu Y, Michelson AD: Results of a worldwide survey on the assessment of platelet function by light transmission aggregometry: a report from the platelet physiology subcommittee of the SSC of the ISTH. J Thromb Haemost. 2009; 7 : 1029-30.

46 Breddin HK: Can platelet aggregometry be standardized? Platelets. 2005;16: 151-8.

47 CaLSI: Platelet function testing by aggregometry; approved guideline. CLSI document H58-A. 2008.

48 Harrison P, Mackie I, Mumford A, Briggs C, Liesner R, Winter M, Machin S: Guidelines for the laboratory investigation of heritable disorders of platelet function. $\mathrm{Br} \mathrm{J}$ Haematol. 2011;155: 30-44.

49 Hayward CP, Pai M, Liu Y, Moffat KA, Seecharan J, Webert KE, Cook RJ, Heddle NM: Diagnostic utility of light transmission platelet aggregometry: results from a prospective study of individuals referred for bleeding disorder assessments. J Thromb Haemost. 2009;7: 676-84.

50 Cardinal DC, Flower RJ: The electronic aggregometer: a novel device for assessing platelet behavior in blood. J Pharmacol Methods. 1980;3: 135-58.

51 Abbate R, Favilla S, Boddi M, Costanzo G, Prisco D: Factors influencing platelet aggregation in whole blood. Am J Clin Pathol. 1986;86: 91-6.

52 Sibbing D, Byrne RA, Bernlochner I, Kastrati A: High platelet reactivity and clinical outcome fact and fiction. Thromb Haemost. 2011;106: 191-202.

53 Toth O, Calatzis A, Penz S, Losonczy H, Siess W: Multiple electrode aggregometry: a new device to measure platelet aggregation in whole blood. Thromb Haemost. 2006;96: 781-8. 
Pittens CA, Bouman HJ, van Werkum JW, ten Berg JM, Hackeng CM: Comparison between hirudin and citrate in monitoring the inhibitory effects of $\mathrm{P}_{2} \mathrm{Y}_{12}$ receptor antagonists with different platelet function tests. J Thromb Haemost. 2009;7: 1929-32.

Kundu SK, Heilmann EJ, Sio R, Garcia C, Davidson RM, Ostgaard RA: Description of an in vitro platelet function analyzer PFA-100. Sem Thromb Hemost. 1995;21 Suppl 2: 106-12. Haubelt $\mathrm{H}$, Anders C, Vogt A, Hoerdt P, Seyfert UT, Hellstern P: Variables influencing platelet function analyzer-100 closure times in healthy individuals. Br J Haematol. 2005;130: 759-67. Harrison P, Robinson MS, Mackie IJ, Joseph J, McDonald SJ, Liesner R, Savidge GF, Pasi J, Machin SJ: Performance of the platelet function analyser PFA-100 in testing abnormalities of primary haemostasis. Blood Coagul Fibrinoly. 1999;10: 25-31. Hayward CP, Harrison P, Cattaneo M, Ortel TL, Rao AK: Platelet function analyzer (PFA)-100 closure time in the evaluation of platelet disorders and platelet function. $\mathrm{J}$ Thromb Haemost. 2006;4: 312-9.

59 Hartert $\mathrm{H}$ : Blutgerinnungsstudien mit der Thromboelastographie. Einem neuen Untersuchungsverfahren. Klin Wochenschr. 1948;26: 577-83.

60 Holzknecht F: Die Auswertungen des Thromboelastogramms. Basel-New York: Karger, S.; 1962. 12-35

61 Escudero J, Mc DE, Wright IS: Dicumarol, coumadin, marcumar and tromexan; comparative study of their action on the clot as registered by the thrombelastogram. Circulation. 1959;20: 405-12.

62 Beller FK: The method for separation of heparin and coumarin effect. Arztliche Wochenschrift. 1954;9: 1159-63.

63 Deutsch E, Fischer M: The effect of intravenously administered streptokinase on fibrinolysis and blood coagulation. Thromb Diathesis Haemorrh. 1960;4: 482-506.

64 Kang YG, Martin DJ, Marquez J, Lewis JH, Bontempo FA, Shaw BW, Starzl TE, Winter PM: Intraoperative changes in blood coagulation and thrombelastographic monitoring in liver transplantation. Anesth Analg. 1985;64: 888-96.

65 Tuman KJ, Spiess BD, McCarthy RJ, Ivankovich AD: Effects of progressive blood loss on coagulation as measured by thrombelastography. Anesth Analg. 1987;66: 856-63.

66 Raviv G, Cramer DB, Epstein M, Zuckerman L, Caprini JA: Electronic readout for threechannel thrombelastograph. J Med. 1978;9: 17-31.

67 Mallett SV, Cox DJ: Thrombelastography. Br J Anaesth. 1992;69: 307-13.

68 Schols SE, Heemskerk JW, van Pampus EC: Correction of coagulation in dilutional coagulopathy: use of kinetic and capacitive coagulation assays to improve hemostasis. Transfu Med Rev. 2010;24: 44-52.

69 Afshari A, Wikkelso A, Brok J, Moller AM, Wetterslev J: Thrombelastography (TEG) or thromboelastometry (ROTEM) to monitor haemotherapy versus usual care in patients with massive transfusion. Cochrane database of systematic reviews. 2011: CD007871. Johansson PI, Stensballe J: Effect of haemostatic control resuscitation on mortality in massively bleeding patients: a before and after study. Vox Sang. 2009;96: 111-8.

71 Schochl H, Nienaber U, Hofer G, Voelckel W, Jambor C, Scharbert G, Kozek-Langenecker S, Solomon C: Goal-directed coagulation management of major trauma patients using thromboelastometry (ROTEM)-guided administration of fibrinogen concentrate and prothrombin complex concentrate. Crit Care. 2010;14: R55.

72 Perry DJ, Fitzmaurice DA, Kitchen S, Mackie IJ, Mallett S: Point-of-care testing in haemostasis. Br J Haematol. 2010;150: 501-14.

73 Ganter MT, Hofer CK: Coagulation monitoring: current techniques and clinical use of viscoelastic point-of-care coagulation devices. Anesth Analg. 2008;106: 1366-75.

74 Holcomb JB, Minei KM, Scerbo ML, Radwan ZA, Wade CE, Kozar RA, Gill BS, Albarado R, McNutt MK, Khan S, Adams PR, McCarthy JJ, Cotton BA: Admission rapid thrombelastography can replace conventional coagulation tests in the emergency department: experience with 1974 consecutive trauma patients. Ann Surg. 2012;256: 476-86.

75 Siller-Matula JM, Christ G, Lang IM, Delle-Karth G, Huber K, Jilma B: Multiple electrode aggregometry predicts stent thrombosis better than the vasodilator-stimulated phosphoprotein phosphorylation assay. J Thromb Haemost . 2010;8: 351-9.

76 Ho AM, Dion PW, Yeung JH, Holcomb JB, Critchley LA, Ng CS, Karmakar MK, Cheung CW, Rainer TH: Prevalence of survivor bias in observational studies on fresh frozen plasma:erythrocyte ratios in trauma requiring massive transfusion. Anesthesiol. 2012;116: 716-28. 
77 Stinger HK, Spinella PC, Perkins JG, Grathwohl KW, Salinas J, Martini WZ, Hess JR, Dubick MA, Simon CD, Beekley AC, Wolf SE, Wade CE, Holcomb JB: The ratio of fibrinogen to red cells transfused affects survival in casualties receiving massive transfusions at an army combat support hospital. J Trauma. 2008;64: S79-85.

78 Hsia CC, Chin-Yee IH, McAlister VC: Use of recombinant activated factor VII in patients without hemophilia: a meta-analysis of randomized control trials. Ann Surg. 2008;248: 61-8.

79 Briedigkeit L, Muller-Plathe O, Schlebusch H, Ziems J: Recommendations of the German Working Group on medical laboratory testing (AML) on the introduction and quality assurance of procedures for point-of-care testing in hospitals. Clin Chem Lab Med. 1999;37: 919-25.

80 Du Plessis M, Ubbink JB, Vermaak WJ: Analytical quality of near-patient blood cholesterol and glucose determinations. Clin Chem. 2000;46: 1085-90.

81 Kitchen DP, Kitchen S, Jennings I, Woods T, Walker I: Quality assurance and quality control of thrombelastography and rotational Thromboelastometry: the UK NEQAS for blood coagulation experience. Sem Thromb Hemost. 2010;36: 757-63.

82 Colucci G, Giabbani E, Barizzi G, Urwyler N, Alberio L: Laboratory-based ROTEM(R)) analysis: implementing pneumatic tube transport and real-time graphic transmission. Int $\mathrm{J} \mathrm{Lab}$ Hematol. 2011;33: 441-6. 


\title{
Chapter 2a
}

Mean platelet volume as a diagnostic marker for cardiovascular disease: drawbacks of pre-analytical conditions and measuring techniques

\author{
Marcus D. Lancé, Matthijs Sloep, \\ Yvonne M.C. Henskens, Marco A.E. Marcus \\ Based on: Journal of Clinical and Applied Thrombosis/Hemostasis \\ 2012 Nov; 18(6):561-8
}




\section{Abstract}

The impedance method has developed to an automated assay integrating mean platelet volume (MPV) measurement as a routine parameter with blood cell counts. Papers have described the MPV as a marker of platelet reactivity and independent risk factor for cardiovascular diseases. This implies knowledge of the pre-analytical variability of this parameter, and the need to have pre-analytical standards. This review shows the high variability in the literature describing MPV as a marker of platlet function in cardiovascular disease. This review further provides an in-depth survey of the methods for MPV measurement and their drawbacks. A possible approach to standardization is proposed. 


\section{Background}

More than one hundred years ago, platelets were called the dust of blood. They were thought to be disintegrated parts from leukocytes or fibrin. In 1882, Bizzozero who was the first to acknowledge the importance of platelets used the term piastrine (small plates). He showed that platelets play a key role in blood coagulation [1, 2]. Since that time, the multiple properties of platelets have been investigated intensively, and a myriad of platelet receptors has been found. In the 1970s, Karpatkin and colleagues were among the first associating platelet size with platelet function [3]. Martin and colleagues showed in the 1980s that larger platelets are increased in reactivity and shorten the bleeding time [4]. Larger platelets were found to express higher levels of P-selectin and glycoprotein IIb/IIla (GPIIb/IIla) [5, 6]. IT was considered that platelet volume may be a risk marker for cardiovascular disease [7]. In about 1000 publications throughout the past decade, mean platelet volume (MPV) has been investigated as a potential marker of platelet reactivity and a surrogate parameter for a broad variety of diseases. Several diseases are strikingly associated with MPV abnormalities. For example, a pathognomonic sign for the Bernard-Soulier syndrome is increased platelet size (also called giant platelets). Also increased platelet turnover is associated with changes in platelet size and function [8]. Large platelets have been associated with cardiovascular disease and prothrombotic states, whereas small platelets are detected in chronic inflammatory diseases, e.g. lupus erythematodes, inflammatory bowel disease and rheumatoid arthritis [9]. This might be explained by the influence of immune mediators and growth hormones on platelet production [10,11].

Accurate measurements of platelet count and size are important for diagnostic, therapeutic and research purposes. Once hematological tests were imprived by the invention of the Coulter principle in the 1950s, size- and countrelated parameters of platelets became easier to determine. In the late 1970s, MPV was added as a standard parameter to the standard set of blood count measurements in automated procedures. However, interpretion of the MPV value thus, using it as a marker in clinical settings - is not straightforward due to drawbacks of the laboratory analysis and the interpretation. Currently, there is no pre-analytical standard for MPV measurements [12].

In the present article, we demonstrate the high technical diversity of measurement types of MPV. After a brief recapitulation of platelet production and 
physiology, we describe the factors contributing to the value of the MPV in more detail. Finally, we give a recommendation to standardize the measurement of MPV.

\section{Literature overview on MPV and cardiovascular disease}

Initially, we conducted a PubMed literature search for papers on MPV. This PubMed search was performed for studies using human subjects, with abstracts in Dutch, German or English, using the following search terms: mean platelet volume (all subheadings) OR MPV (all subheadings) OR platelet sizing (all subheadings) AND cardiovascular diseases $(\mathrm{MeSH})$ OR atherosclerosis $(\mathrm{MeSH})$ OR coronary disease (MeSH) OR hypertension (MeSH) OR myocardial infarction (MeSH). Due to the vast amount of resulting publications, the search criteria were then limited to MPV publications related to cardiovascular disease between 2006 and 2011. Cardiovascular disease was selected as interest area, because many studies discuss this in relation with MPV [13]. For instance, reports describe MPV as a risk factor for myocardial infarction, coronary artery disease and stroke [14, 15].

The limited search resulted in 126 articles, 63 of which were not directly about the MPV measurement method, and were excluded. Full texts of the remaining 63 articles were analyzed, and methodological design of the studies was assessed in three ways: type of anticoagulant used for blood drawing tubes; differences in time delay between blood drawing and MPV measurement with ethylenediamine tetraacetic acid (EDTA) anticoagulant after 120 minutes and with citrate after 60 minutes [16, 17]; and cell count device or method used.

Two articles were excluded: one was a meta-analysis and one a review. The remaining 61 articles mentioned at least one of the methodological items. More than half of the authors $(62 \%, n=39 / 61)$ were aware of technical drawbacks of their methods, and they described all three analyzed variables (anticoagulant type, time interval of measurement, and counting method) (Figure 1). The majority of the authors did not present solutions for standardizing these variables. More specifically, $82.5 \%(n=52 / 61)$ of the studies reported the type of anticoagulant which used but not the concentration (for heparin, EDTA and citrate: 1, 41 and 10 studies, respectively). In $65 \%(n=41 / 61)$ of the studies, the time interval between blood sampling and measurement was given, but only $3.2 \%(n=2 / 61)$ followed the general recommendation $[16,17]$ to measure after 120 minutes in EDTA anticoagulant, and 
Matched search string

\section{6}

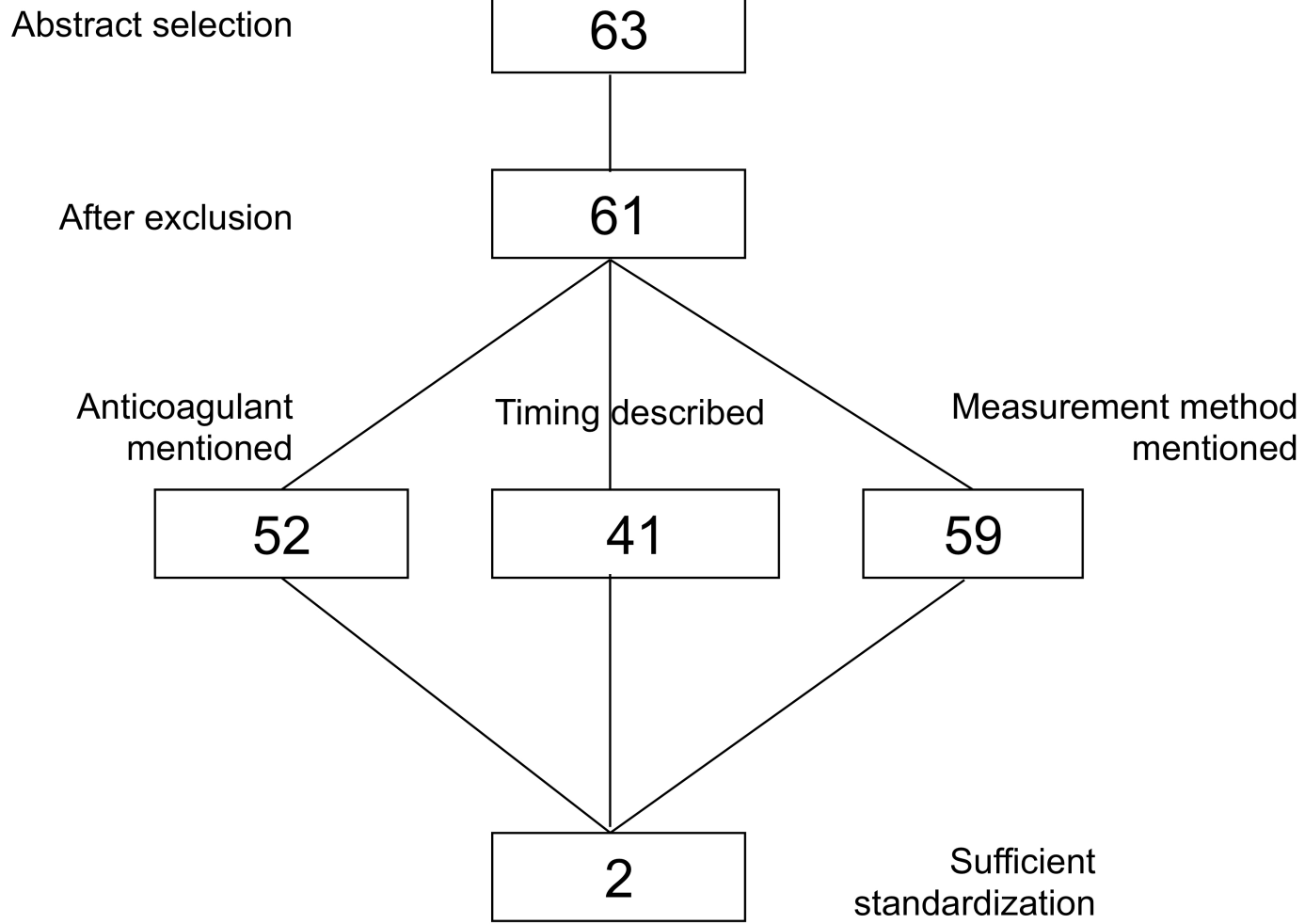

Figure 1: Search strategy: 126 articles met the initial search strategy criteria, 63 dealt with MPV measurement methods, 2 papers were excluded (a review and meta-analysis). Furthermore, 52 papers described the anticoagulant used, 41 included the time interval to measurement, and 59 reported on the detection method. Only 2 articles informed on all standardization criteria (anticoagulant, time interval for measurement, and detection method).

after 60 minutes in citrate medium. More than ten different descriptions of the time to measurement were present, ranging from immediately after blood sampling to within 24 hours after sampling, and almost any interval in between. Finally, 93.7\% ( $\mathrm{n}=$ 59/61) mentioned the device used for measurement. All kinds of techniques were used for this measurement. This high variability causes confusion and makes comparisons difficult. 


\section{Platelet production}

The intra-individual platelet count is normally maintained within a narrow range during a person's life, whereas the average platelet count between individuals can differ up to three-fold (150 to $450 \times 10^{9} /$ liter) [18]. Nevertheless, the total platelet mass, which is the product of platelet count and MPV, is normally fairly constant [19]. In general the platelet count is inversely related to the MPV. Hence, in patients with low platelet count the MPV is larger and vice versa [16, 20,21]. The normal range of platelet count can be defined as the mean \pm 2 SD of the count of a group of healthy individuals [22].

An adult generates approximately $10^{11}$ new platelets per day that have a lifespan of about eight to ten days. The production may increase up to tenfold in the event of higher demands for platelets (e.g. at blood loss) [11]. Under these conditions, the MPV of freshly released platelets is increased within eight hours after onset of thrombocytopenia [23]. However, the relation between platelet size and platelet age is still being debated. Some authors argue that platelets decrease in size due to aging, whereas others do not support this theory. It is known that platelet size decreases as a result of chemotherapy and radiation. The mechanism of this is unclear. It may be due to the therapy (alteration of thrombopoiesis) or to an effect of selective consumption of larger platelets. The latter explanation is valid for surgical and trauma patients [16, 24].

The spleen plays a central role in platelet homeostasis. It removes old platelets from the circulation. Moreover, the spleen stores one third of the total platelet number, while its release depends on adrenergic stimuli [25]. The amount of storage may increase up to 85 to $90 \%$ of the total platelets in case of splenomegaly $[26,27]$. On the other hand, platelet size decreases in inflammatory diseases such as systemic lupus erythemadodes, inflammatory bowel disease and rheumatoid arthritis [9]. This might be a result of the delicate interaction of thrombopoietin and immune mediators on platelet production. It is known that tumor necrosis factor alpha, interleukins 1 and 6, and granulocyte-macrophage colony-stimulating factor affect megakaryocyte proliferation and platelet count [9-11]. 


\section{Platelet physiology}

Besides their well-known function in hemostasis, platelets also play a role in inflammatory and immune-modulatory processes. Their surface holds a variety of glycoprotein receptors. The presence of glycoprotein Ilb/llla (GPIIb/llla) is unique to platelets, and its absence is indicative for Glanzmann's thrombasthenia. Important for hemostasis is also the GPIb-V-IX complex, the absence of which is a pathognomonic sign for Bernard-Soulier syndrome. In addition, the platelet membrane contains receptors for thrombin, ADP, collagen, thromboxane and many other plateletactivating substances.

Platelets contain secretory granules, which are release during coagulation in hemostasis. The most numerous are alpha granules, in which fibrinogen, von Willebrand factor (VWF), thrombospondin and fibronectin are stored. Their number depends on the size of the platelets, but is about ten times higher than the number of dense granules [28]. Disease-specific changes in granular number are the giant alpha granules, associated Paris-Trousseau/Jacobsen syndrome and the hypogranular, White platelet syndrome. Degranulation of alpha granules is an early sign of storage injury together with surface exposure of P-selectin [29].

Normally, three to eight dense granules can be found per platelet. They contain ADP, ATP, GTP, serotonin and 60 to $70 \%$ of whole platelet calcium [30]. Further, platelets contain lysosomes, mitochondria, endoplasmatic reticulum and a microtubular system. There may be a link between platelet size and overall platelet content. Larger platelets are supposed to contain more granules and hereby become more reactive. However, there is no consensus about the relationship between platelet size and platelet age, but large platelets are considered to have more hemostatic capacity [19, 31, 32].

The role of platelets in thrombus formation can be briefly described as a sequence of several phases. In the resting phase, platelets have a discoid shape of 2 to $4 \mu \mathrm{m}$ in diameter. Platelets then become activated by interaction of their receptors with specific ligands. Interaction of GPIb-V-IX with immobilized VWF leads to adhesion and shape change of flowing platelets. The occupation of GPIb-V-IX also stimulates GPIIb/llla to bind fibrinogen. Fibrinogen-bound GPIIb/llla facilitates the bridging of platelets to form an aggregate and supoorts the stabilization of a clot [33, 34]. Platelet interaction with collagen via GPVI greatly supports platelet activation in the early phase of thrombus formation [34]. Consequence of the signaling pathways 
induced by GPIb-V-IX and GPVI is secretion of the dense granules and alpha granules. The secreted products boost further platelet activation and promote a role of platelets in the coagulation system [35].

\section{Platelet count and MPV measurement}

The literature describes four different principles for platelet counting and MPV measurement: manual counting by phase contrast microscopy, impedance counting, optical light scatter counting, and immunological flow cytometry techniques.

Manual counting by phase-contrast microscopy of platelets was considered the gold standard until very recently [36]. Phase-contrast microscopy uses microchambers in which the platelets are counted manually and their size is estimated with a reference grid. For a long time, this work-intensive technique did not allow for precise measurement of the MPV.

Although the implementation of automated counters in the 1970s increased precision of measurements, problems remained in differentiating between platelets and other small particles, such as fragmented red cells and immune complexes, especially in patients with low platelet count. As a result, manual microscopy continued to be the international reference method, and manual counting was used for calibration of the automated systems. Nevertheless, there are concerns about the imprecision of manual counting, with an inter-observer variability of 10 to $25 \%$ [37].

In the 1950s, Coulter developed and presented the Coulter principle using electronic impedance, which revolutionized the method of platelet counting [38-40]. The impedance method is based on the conduction properties of cells. A suspension of blood diluted in isotonic saline is passed through a small aperture. A current flows through this aperture and as a given cell passes through, the resistance increases. The poor conductive property of a cell thus increases the impedance, which is measured by an increase in voltage or pulse. In theory, the number of pulses corresponds with the number of non-conductive particles that pass through, while the amplitude of the pulses corresponds with the volume of the particles.

Two problems that occur are the measurement of two simultaneously passing particles and the erroneous measurement of (fragmented or small) red blood cells. Upon impedance counting, particles ranging from 2 to $20 \mathrm{fL}$ in volume are considered to be platelets. This range is applicable in most cases, since there are no more than few small red blood cells, giant platelets and cell fragments. From all the platelets 
that are counted, a platelet size histogram is created and extrapolated to a volume of $70 \mathrm{fL}$, after which log-normal curves are used to fit this histogram. The output data are calculated from this superimposed curve, in which the MPV corresponds with the geometric mean. Fitting of the curves is needed to exclude microcytic red cells, and it allows the inclusion of giant platelets.

Because of the above-mentioned problems with impedance measurements, another automated technique was considered to be superior. Optical cell counting was introduced in the 1970s. In this technique, cells are focused in a flow cell and its light scatter is measured after illumination with a laser. In order to obtain information on count and size of the cells, the light scatter is measured at two angles and converted to electrical pulses. Similar to the impedance technology, the number of pulses corresponds with the cell count, and the amplitude is proportional to the cell volume. A platelet histogram is also produced from these measurements, but no lognormal curve is generated. The MPV is calculated from the geometrical mode instead of the geometrical mean, which means that it is derived from the most frequent volume in the distribution curve. Optical fluorescence platelet counting is a variation of this method. Here, reticulated cells and platelet membranes are first stained with a fluorescent label. The intensity of fluorescence allows platelets, including giant platelets, to be selected. As with the impedance measurement, MPV is measured from the mode, instead of the calculated mean [41]. The differences between optical and impedance measurement techniques may result in different MPV values for the same sample. Differences of up to $40 \%$ have been reported [42-44].

In 2000, Harrison et al. presented a method for immuno platelet counting. The high precision of this method prompted the authors to propose this as new reference technique [37]. Briefly, immunological measurement uses monoclonal antibodies against platelet surface antigens (mainly anti-CD41 and anti-CD61 mAbs). These are covalently labeled with fluorescent probes, such as fluorescein isothiocyanate. The antigens are detected from their fluorescence when passing the detection cell of a flow cytometer. Platelet counts are determined by adding in addition a fixed number of fluorescent-labeled calibration beads, and larger platelets are detected from the higher fluorescence. Due to the high costs and the inaccessibility of the flow cytometer technique for some laboratories, this method is not to be considered a standard or routine assay. Newer fully automated immunological methods have come on the market, but their use is not yet widespread. The main advantage of the 
immuno platelet counting method is high accuracy, especially in samples with low platelet counts. However, there are also some concerns. Harrison and colleagues mentioned that their method might overestimate values in patients who have high levels of anti-platelet antibodies or who are treated with anti-platelet glycoprotein agents (e.g. anti-GPIIb/IIla antibodies) [37]. An underestimation of the actual platelet count could be obtained in absence of platelet receptors for the target antigen, for example, in Bernard-Soulier syndrome. Sandhaus and colleagues observed a mild underestimation of the platelet count due to the presence of large platelets or platelet clumps [45]. In spite of this, the method is considered to be highly accurate, particularly when patients are thrombocytopenic; making it a possible new gold standard and recommending its use for this population [46, 47].

Next to the MPV, another parameter - the mean platelet component (MPC) was introduced by Macey et al. as a surrogate parameter for platelet activation [48]. This parameter is obtained in automated flow cytometric measurements using the ADVIA system. The authors reported an inverse relation between CD62 expression as a marker of platelet activation and the MPC. However, in samples with EDTA as anticoagulant, the MPC results were not stable. In contrast to MPV, the MPC slightly increased during the first hour and then decreased up to 24 hours [49]. In a recent article, Cooke and co-workers did not detect a positive correlation between MPC and the severity of coronary artery disease [50]. However, Diaz-Ricart reported on additives to EDTA to make the results for MPC more stable [49]. In this light, MPC as a parameter for platelet activation seems interesting, but currently not recommendable as standard parameter.

\section{Factors influencing the value of MPV}

Apart from methodological aspects affecting the results, some pre-analytical variables are also important to mention. Notably, the method of venipuncture and the degree of accuracy of filling and gently mixing the blood sampling tubes may affect platelet activation and result in aggregate formation. The choice of anticoagulant, time interval of the measurement and temperature, at which MPV is analyzed, are also relevant. In general, for all hematological laboratory tests, and hence for blood cell counting, the blood sample needs to be anticoagulated to prevent formation of a fibrin clot. Commonly, this is achieved with either EDTA or sodium citrate as standard anticoagulants. Both bind $\mathrm{Ca}^{2+}$ ions, thereby preventing coagulation in an irreversible 
(EDTA) or reversible (sodium citrate) manner. Some other substances are not used standardly, such as theophylline or wortmannin (Table 1). Regardless of the anticoagulant medium, platelets will swell for some time. The mechanism of swelling is not completely known, but it is probably a consequence of chelation of membranebound $\mathrm{Ca}^{2+}$ on platelets [51]. In particular, EDTA causes morphological changes of platelets. The canalicular system opens, the platelet volume increases and the GPIIb/IIla molecules dissociate irreversibly [52-54].

Unfortunately, the platelet reaction in anticoagulants is not uniform. For example, Na-EDTA as an anticoagulant results in less pronounced swelling than KEDTA [55]. Between laboratories even the concentration of anticoagulant may vary, which for instance results in more distinct swelling in "low concentration" citrate $(0.12$ $\mathrm{M}$; ratio blood to citrate 9:1) and almost no swelling in "high concentration" citrate (0.12 M, ratio blood to citrate 4:1) [16]. Thompson and Trowbridge were the first, who directly compared the effects of different anticoagulants on platelet swelling $[42,55]$. Both proposed to use standard anticoagulant media and fixed time points after sampling for the most reproducible measurement of MPV. Since that time, many other authors reported effects with different anticoagulants (Table 1). In 1993, the International Council for Standardization in Hematology published a recommendation on this. It was proposed to use K-EDTA $(1.5-2.2 \mathrm{mg} / \mathrm{mL}$ blood) as a reference method. However, many other anticoagulant media are still in use in clinical and investigational settings [56].

Another important issue is the time interval of the measurement. As already mentioned, Thompson and Bath reported on platelet swelling [16, 55]. Effects of the time interval were negligible at a high concentration Na-citrate (citrate to blood, 1:4), whereas in low concentration Na-citrate (citrate to blood, 1:9) and K-EDTA media, most stable results were seen after 60 and 90 minutes, respectively. Others recommend to measure within 1 hour regardless of the type of anticoagulant [21]. Finally, some authors suggest using additives for accurate measurement $[49,57]$.

In a recent study, our group recommended a reference interval for time-adjusted MPV measurements for two common standard media, K-EDTA and Na-citrate, using the Coulter LH 750. The aim was to obtain a simple standardization of the MPV measurement with commonly used anticoagulants [17]. It was concluded that an optimal stability of the MPV measurement with K-EDTA medium was reached after 120 minutes and in Na-citrate medium after 60 minutes. 


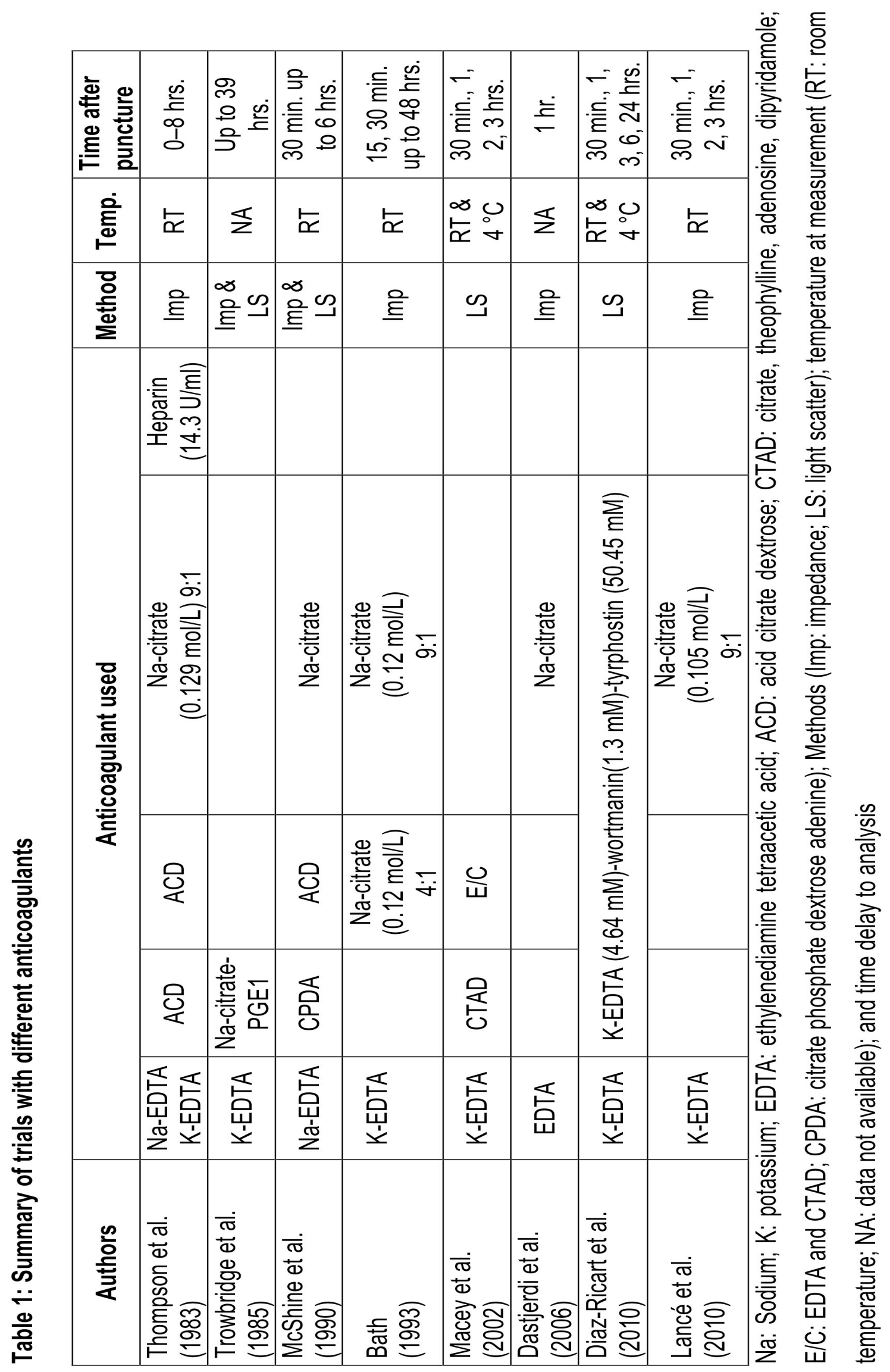


This resulted in a reference range of 7.2-10.8 $\mathrm{fL}$ with K-EDTA and 6.1-9.5 fL with Na-citrate. Recently, we also determined MPV reference ranges for the Sysmex XE-5000 apparatus. In K-EDTA medium after 120 minutes, this reference range was different from that with the Coulter LH 750, namely 9.2-12.7 $\mathrm{fL}(\mathrm{n}=120)$ (unpublished data). This indicates that MPV results are not only dependent on time but also on the method and device.

Another pre-analytical condition, the storage temperature of sample tubes, has been the subject of only two publications. Both studies showed that cooling down citrate-anticoagulated blood samples from $37^{\circ} \mathrm{C}$ to $4{ }^{\circ} \mathrm{C}$ increases MPV values by $18 \%[58,59]$. Even though this temperature effect is mentioned more frequently, it is not common knowledge [8]. In daily practice, MPV measurements are performed at room temperature.

A limitation of this review is that there is not much literature on pre-analytical differences in blood-drawing techniques nor on their influence on MPV values. Furthermore, in spite of the relevance of the blood sampling method for standard laboratory tests, their impact on platelet function analysis was recently reported to be minor [60,61]. There are no reports on the influence of specimen transport on MPV. However, such influence likely is minimal, as there is only a small change due to pneumatic tube transport on standard blood parameters in the laboratory [62, 63]. Finally, the literature search was limited to PubMed and cardiovascular disease in association with MPV. Yet, due to the large number of papers found at this restriction, the current analysis likely is a good reflection of the overall literature.

\section{Conclusions}

It is difficult to compare MPV results obtained by different measurement methods, unless correction coefficients have been determined. Because the use of MPV as a diagnostic marker is increasingly popular, it is important that a standard method is agreed upon. This is important to be able to compare measurements from different studies. A standard method should include definitions of the temperature, the (commonly used) anticoagulant, the time delay between blood sampling and measurement, and the technique and equipment used. For hospital laboratories, this should facilitate the measurement of MPV as a routine parameter. As long as this standard method is not operative, each laboratory should determine reference values, considering the aforementioned recommendations. 


\section{References}

1 Mazzarello P, Calligaro AL, Calligaro A: Giulio Bizzozero: a pioneer of cell biology. Nat Rev Mol Cell Biol. 2001;2: 776-81.

2 Brewer DB: Max Schultze (1865), G. Bizzozero (1882) and the discovery of the platelet. $\mathrm{Br} \mathrm{J}$ Haematol. 2006;133: 251-8.

3 Karpatkin S, Freedman ML: Hypersplenic thrombocytopenia differentiated from increased peripheral destruction by platelet volume. Ann Intern Med. 1978;89: 200-3.

4 Martin JF, Trowbridge EA, Salmon G, Plumb J: The biological significance of platelet volume: its relationship to bleeding time, platelet thromboxane $\beta_{2}$ production and megakaryocyte nuclear DNA concentration. Thromb Res. 1983;32: 443-60.

5 Karpatkin S, Khan Q, Freedman M: Heterogeneity of platelet function. Correlation with platelet volume. Am J Med. 1978;64: 542-6.

6 Breimo ES, Osterud B: Studies of biological functions in blood cells from individuals with large platelets. Platelets. 2003;14: 413-9.

7 Tsiara S, Elisaf M, Jagroop IA, Mikhailidis DP: Platelets as predictors of vascular risk: is there a practical index of platelet activity? Clin Appl Thromb Hemost. 2003;9: 177-90.

8 Jackson SR, Carter JM: Platelet volume: laboratory measurement and clinical application. Blood Rev. 1993;7: 104-13.

9 Gasparyan AY, Ayvazyan L, Mikhailidis DP, Kitas GD: Mean platelet volume: a link between thrombosis and inflammation? Current Pharma Des. 2011;17: 47-58. Kaushansky K: The molecular mechanisms that control thrombopoiesis. J Clin Invest. 2005;115: 3339-47.

11 Kaushansky $\mathrm{K}$ : Determinants of platelet number and regulation of thrombopoiesis. Hematology : the Education Program of the American Society of Hematology American Society of Hematology Education Program. 2009: 147-52.

12 Buttarello M, Plebani M: Automated blood cell counts: state of the art. Am J Clin Pathol. 2008;130: 104-16.

13 Cameron HA, Phillips R, Ibbotson RM, Carson $\mathrm{PH}$ : Platelet size in myocardial infarction. $\mathrm{Br}$ Med J. (Clin Res Ed). 1983;287: 449-51.

14 Chu SG, Becker RC, Berger PB, Bhatt DL, Eikelboom JW, Konkle B, Mohler ER, Reilly MP, Berger JS: Mean platelet volume as a predictor of cardiovascular risk: a systematic review and meta-analysis. J Thromb Haemost. 2009;8: 148-56.

Vizioli L, Muscari S, Muscari A: The relationship of mean platelet volume with the risk and prognosis of cardiovascular diseases. Int J Clin Pract. 2009;63: 1509-15.

16 Bath PM: The routine measurement of platelet size using sodium citrate alone as the anticoagulant. Thromb Haemost. 1993;70: 687-90.

Lancé MD, van Oerle R, Henskens YM, Marcus MA: Do we need time adjusted mean platelet volume measurements? Lab Hematol. 2010;16: 28-31.

18 Giles C: The platelet count and mean platelet volume. Br J Haematol. 1981;48: 31-7.

19 Thompson CB, Jakubowski JA: The pathophysiology and clinical relevance of platelet heterogeneity. Blood. 1988;72: 1-8.

20 O'Brien JR: Letter: A relationship between platelet volume and platelet number. Thromb Diath Haemorrh. 1974;31: 363-5.

21 Dastjerdi MS, Emami T, Najafian A, Amini M: Mean platelet volume measurement, EDTA or citrate? Hematol. 2006;11: 317-9.

22 Szalai G, LaRue AC, Watson DK: Molecular mechanisms of megakaryopoiesis. Cell Mol Life Sci. 2006;63: 2460-76.

23 Corash L, Chen HY, Levin J, Baker G, Lu H, Mok Y: Regulation of thrombopoiesis: effects of the degree of thrombocytopenia on megakaryocyte ploidy and platelet volume. Blood. 1987;70: 177-85.

24 Thompson CB: Selective consumption of large platelets during massive bleeding. Br Med J. (Clin Res Ed). 1985;291: 95-6.

25 Branehog I, Weinfeld A, Roos B: The exchangeable splenic platelet pool studied with epinephrine infusion in idiopathic thrombocytopenic purpura and in patients with splenomegaly. Br J Haematol. 1973;25: 239-48.

26 Aster RH: Platelet sequestration studies in man. Br J Haematol. 1972;22: 259-63.

27 Penny R, Rozenberg MC, Firkin BG: The splenic platelet pool. Blood. 1966;27: 1-16.

28 Blair P, Flaumenhaft R: Platelet alpha-granules: basic biology and clinical correlates. Blood Rev. 2009;23: 177-89. 
Keuren JF, Cauwenberghs S, Heeremans J, de Kort W, Heemskerk JW, Curvers J: Platelet ADP response deteriorates in synthetic storage media. Transfusion. 2006;46: 204-12.

McNicol A, Israels SJ: Platelet dense granules: structure, function and implications for haemostasis. Thromb Res. 1999;95: 1-18.

31 Schoene NW: Design criteria: tests used to assess platelet function. Am J Clin Nutr. 1997;65: 1665S-8S.

Thompson CB, Jakubowski JA, Quinn PG, Deykin D, Valeri CR: Platelet size and age determine platelet function independently. Blood. 1984;63: 1372-5.

George JN: Platelets. Lancet. 2000;355: 1531-9.

Andrews RK, Berndt MC: Platelet physiology and thrombosis. Thromb Res. 2004;114: 447-53. Kickler TS: Platelet biology: an overview. TATM. 2006;8: 79-85.

Brecher G, Schneiderman M, Cronkite EP: The reproducibility and constancy of the platelet count. Am J Clin Pathol. 1953;23: 15-26.

Harrison P, Horton A, Grant D, Briggs C, MacHin S: Immunoplatelet counting: a proposed new reference procedure. $\mathrm{Br}$ J Haematol. 2000;108: 228-35.

Briggs C, Harrison P, Machin SJ: Continuing developments with the automated platelet count. Int J Lab Hematol. 2007;29: 77-91.

Lehner J, Greve B, Cassens U: Automation in hematology. Transfusion Med Hemoth. 2007;34: 328-39.

Coulter WH: Apparatus for studying the
liquid. US Patent. 1955; No. 15166/51. Briggs C, Harrison P, Grant D, Staves J, MacHin SJ: New quantitative parameters on a recently introduced automated blood cell counter: the XE 2100. Clin Lab Haematol. 2000;22: 345-50.

Trowbridge EA, Reardon DM, Hutchinson D, Pickering C: The routine measurement of platelet volume: a comparison of light-scattering and aperture-impedance technologies. Clin Phys Physiol Meas. 1985;6: 221-38.

Vagdatli E, Gounari E, Lazaridou E, Katsibourlia E, Tsikopoulou F, Labrianou I: Platelet distribution width: a simple, practical and specific marker of activation of coagulation. Hippokratia. 2010;14: 28-32.

44 Latger-Cannard V, Hoarau M, Salignac S, Baumgart D, Nurden P, Lecompte T: Mean platelet volume: comparison of three analysers towards standardization of platelet morphological phenotype. Int J Lab Hematol. 2012;34: 300-10.

45 Sandhaus LM, Osei ES, Agrawal NN, Dillman CA, Meyerson HJ: Platelet counting by the coulter LH 750, sysmex XE 2100, and advia 120: a comparative analysis using the $\mathrm{RBC}$ /platelet ratio reference method. Am J Clin Pathol. 2002;118: 235-41.

46 Grimaldi E, Del Vecchio L, Scopacasa F, Lo Pardo C, Capone F, Pariante S, Scalia G, De Caterina M: Evaluation of the platelet counting by Abbott CELL-DYN SAPPHIRE haematology analyser compared with flow cytometry. Int J Lab Hematol. 2009;31: 151-60.

47 Platelet counting by the RBC/platelet ratio method. A reference method. Am J Clin Pathol. 2001;115: 460-4.

48 Macey MG, Carty E, Webb L, Chapman ES, Zelmanovic D, Okrongly D, Rampton DS, Newland AC: Use of mean platelet component to measure platelet activation on the ADVIA 120 haematology system. Cytometry. 1999;38: 250-5.

49 Diaz-Ricart M, Brunso L, Pino M, Navalon F, Jou JM, Heras M, White JG, Escolar G: Preanalytical treatment of EDTA-anticoagulated blood to ensure stabilization of the mean platelet volume and component measured with the ADVIA counters. Thromb Res.126: e30-5.

50 Cooke J, Murphy T, McFadden E, O'Reilly M, Cahill MR: Can mean platelet component be used as an index of platelet activity in stable coronary artery disease? Hematology. 2009;14: $111-4$.

51 White JG: Effects of ethylenediamine tetracetic acid (EDTA) on platelet structure. Scand J Haematol. 1968;5: 241-54.

52 Gachet C, Hanau D, Spehner D, Brisson C, Garaud JC, Schmitt DA, OhImann P, Cazenave JP: $\alpha_{\| 1 b} \beta_{3}$ integrin dissociation induced by EDTA results in morphological changes of the platelet surface-connected canalicular system with differential location of the two separate subunits. J Cell Biol. 1993;120: 1021-30.

53 White JG: EDTA-induced changes in platelet structure and function: clot retraction. Platelets. 2000;11: 49-55.

54 White JG, Escolar G: EDTA-induced changes in platelet structure and function: adhesion and spreading. Platelets. 2000;11: 56-61. 
Thompson CB, Diaz DD, Quinn PG, Lapins M, Kurtz SR, Valeri CR: The role of anticoagulation in the measurement of platelet volumes. Am J Clin Pathol. 1983;80: 327-32. Recommendations of the international council for standardization in haematology for ethylenediaminetetraacetic acid anticoagulation of blood for blood cell counting and sizing. International council for standardization in haematology: expert panel on cytometry. Am J Clin Pathol. 1993;100: 371-2.

57 Macey M, Azam U, McCarthy D, Webb L, Chapman ES, Okrongly D, Zelmanovic D, Newland A: Evaluation of the anticoagulants EDTA and citrate, theophylline, adenosine, and dipyridamole (CTAD) for assessing platelet activation on the ADVIA 120 hematology system. Clin Chem. 2002;48: 891-9.

58 Park Y, Schoene N, Harris W: Mean platelet volume as an indicator of platelet activation: methodological issues. Platelets. 2002;13: 301-6.

59 Threatte GA, Adrados C, Ebbe S, Brecher G: Mean platelet volume: the need for a reference method. Am J Clin Pathol. 1984;81: 769-72.

60 Lippi G, Banfi G, Buttarello M, Ceriotti F, Daves M, Dolci A, Caputo M, Giavarina D, Montagnana M, Miconi V, Milanesi B, Mosca A, Morandini M, Salvagno GL: Recommendations for detection and management of unsuitable samples in clinical laboratories. Clin Chem Lab Med. 2007;45: 728-36.

61 Lancé MD, Henskens YM, Nelemans P, Theunissen MH, van Oerle R, Spronk HM, Marcus MA: Do blood collection methods influence whole-blood platelet function analysis? Platelets. 2012.

62 Keshgegian AA, Bull GE: Evaluation of a soft-handling computerized pneumatic tube specimen delivery system. Effects on analytical results and turnaround time. Am J Clin Pathol. 1992;97: 535-40.

63 Lancé MD, Kuiper GJ, Sloep M, Spronk HM, van Oerle R, Ten Cate H, Marcus MA, Henskens YM: The effects of pneumatic tube system transport on ROTEM analysis and contact activation assessed by thrombin generation test. Thromb Res. 2012, Sep;130(3):e147-50. 


\section{Chapter 2b}

Do we need time-adjusted mean platelet volume measurements?

Marcus D. Lancé, René van Oerle,

Yvonne M.C. Henskens, Marco A.E. Marcus

Based on: Laboratory Hematology 2010, 16:28-31 


\begin{abstract}
Changed mean platelet volume (MPV) has been associated with various diseases. Several authors reported dependency of MPV values of anticoagulant type and timeto-measurement. Therefore, standardized laboratory methods for this blood parameter are essential. The aim of this study was to achieve such standardization. Blood from 20 healthy volunteers was collected in tubes containing potassium ethylenediaminotetra acid (EDTA) or sodium citrate. Platelet count and MPV were determined every half hour for 4 hours. The same parameters under optimal conditions were obtained with blood from a second group of 100 healthy donors to determine a reference range. Citrate-anticoagulated samples gave a significantly lower MPV $(7.0 \pm 0.69 \mathrm{fL}, \mathrm{SD})$ than EDTA-anticoagulated blood $(8.0 \pm 0.8 \mathrm{fL})$. The MPV value increased during 120 minutes in the presence of EDTA and during 60 minutes with citrate. Platelet count changed significantly during this time in the presence of citrate. In the second group, an inverse correlation between MPV and platelet count was not detected. Reference range calculated for EDTA- and citrateanticoagulated blood were 7.2-10.8 fL and 6.1-9.5 fL, respectively, thus pointing to lower MPV values of platelets in citrate medium.
\end{abstract}




\section{Introduction}

Platelets have key functions in arterial thrombus formation and hemostasis. Platelet activity is frequently measured because of the therapeutic impact of blocking this part of the hemostatic system. A simple determination of platelet function is also important for decision-making in hemorrhagic patients, e.g. with respect to choice of the anesthesia procedure. Mean platelet volume (MPV) has been put forward as an easily obtainable proxy parameter for platelet activity. Since the introduction of the automated measurement of MPV in the early 1980s [1], this parameter now sees a revival.

There are increasing indications that the measured value of the MPV differs with the choice of anticoagulant, concentration of anticoagulant, and time to measurement. Bath and Thompson have tried to standardize the method by determining the optimal medium for this measurement [2, 3]. They used ethylenediaminetetra acid (EDTA) and sodium citrate anticoagulants at different concentrations. Other authors have published effects caused by different time intervals between blood drawing and measurement [4]. Given that the preanalytical conditions of the MPV measurement in many papers are not described or even recorded, standardization of the measurement of this parameter is highly needed.

There are a number of indications that the MPV relates to activity of the platelets. A large MPV is associated with higher density of granules, higher thromboxane $A_{2}$ production, and increased expression of glycoprotein $\mathrm{Ib} / \mathrm{V} / \mathrm{IX}$ and glycoprotein Ilb/IIla [5-7]. In vitro studies revealed higher aggregability of platelets with bigger MPV values. This has led to the suggestion that an increased platelet volume leads to increased platelet reactivity [8]. In addition, there exists an inverse relation between the platelet count and the MPV $[2,3,9$, 10]. Several attempts have thus been made to correlate the MPV with the state of different diseases such as acute haemorrhage, coronary artery disease, arterial hypertension, pregnancyassociated diseases, metabolic disorders including diabetes mellitus, and many more [11-17]. A regular conclusion was that a large MPV correlates with high platelet activity. To substantiate this, it is highly needed to come to a uniform method for testing of this blood parameter.

In the present study we aimed to come to a standardization of the measurement of the MPV with regard to commonly used anticoagulants and usual 
times to measurement. We also made a standard reference range for the MPV parameter at appropriate conditions.

\section{Methods}

After given written informed consent, 20 healthy volunteers were included for defining the optimal measurement time interval of MPV measurement in blood collected in two different anticoagulant media (K-EDTA and Na-citrate). Another 100 volunteers gave informed consent for measurement of the MPV under specific conditions, in order to obtain a range of reference values for blood collected into K-EDTA and Na-citrate. None of the volunteers took any medication within the previous 12 days. Volunteers taking nonsteroidal analgesics such as acetylic acid were excluded from the study. Collection of blood was done by tourniquet use at standard conditions. The first $5 \mathrm{~mL}$ of blood were discarded.

For the first part of the study (20 donors), blood was collected into 2 vacuum containers (K-EDTA $7.2 \mathrm{mg} / 4 \mathrm{~mL}$, and Na-citrate $0.105 \mathrm{M}$ at 1:10 vol.; both $\mathrm{BD}$ Vacutainer, Plymouth, UK) and gently mixed. Platelet count and MPV were measured immediately after drawing, and after time intervals of 0.5 hour up to 4 hours, using the Coulter LH-750 Hematology Analyzer (Beckman Coulter, Brea, CA, USA). This resulted in 9 measurement points (Table 1). The measuring principle for MPV of this machine is based on changes in impedance. Cells are counted as numbers of pulses, while the cell volume is a function of the strength of those pulses.

In the second part, we measured MPV in blood from 100 healthy volunteers at 120 minutes (K-EDTA) and 90 minutes (Na-citrate) after venipuncture to determine reference ranges.

Statistical analyses were performed using SPSS version 17 (SPSS, Chicago, IL, USA). We used t-tests for comparing differences between samples, and one-way ANOVA with Bonferroni correction for different times.

\section{Results}

In the first part of the study, in blood from 20 healthy subjects, we initially measured a MPV of $8.0 \pm 0.8 \mathrm{fL}$ (mean \pm SD) in K-EDTA anticoagulant, and of $7.0 \pm 0.69 \mathrm{fL}$ in Na-citrate anticoagulant $(P=0.0001)$. During the period of 240 minutes the MPV 
Table 1: MPV (fL) and mean platelet count $\left(\times 10^{9} / \mathrm{L}\right)$ at indicated times after blood collection into KEDTA or Na-citrate. Data are means (SD), $n=20$.

\begin{tabular}{lcccc} 
& EDTA & Citrate & EDTA & Citrate \\
\cline { 2 - 5 } Time $(\min )$ & MPV $(\mathrm{fL})$ & MPV $(\mathrm{fL})$ & Platelet count & Platelet count \\
\hline 0 & $8.0(0.8)$ & $7.0(0.69)$ & $264(44.4)$ & $271(43.7)$ \\
30 & $8.6(0.76)$ & $7.7(0.85)$ & $262(44.5)$ & $269(44.6)$ \\
60 & $8.9(0.74)$ & $7.9(0.86)$ & $261(42.2)$ & $262(42.6)$ \\
90 & $9.0(0.78)$ & $8.0(0.81)$ & $261(43.3)$ & $257(39.9)$ \\
120 & $9.1(0.75)$ & $8.1(0.84)$ & $260(43.5)$ & $256(40.9)$ \\
150 & $9.1(0.76)$ & $8.0(0.84)$ & $260(43.2)$ & $250(42.7)$ \\
180 & $9.2(0.79)$ & $8.0(0.86)$ & $261(43.9)$ & $243(40.8)$ \\
210 & $9.2(0.84)$ & $8.0(0.83)$ & $260(43.4)$ & $242(43)$ \\
240 & $9.3(0.79)$ & $8.0(0.78)$ & $261(44.1)$ & $237(36.5)$ \\
\hline
\end{tabular}

increased to $9.3 \pm 0.79 \mathrm{fL}(P=0.0001)$ in EDTA and $8.0 \pm 0.78 \mathrm{fL}(P=0.0001)$ in citrate anticoagulant (Table 1).

Platelet swelling in Na-citrate continued up to 60 minutes, and then decreased to to about $0.1 \mathrm{fL}$ per hour (Figure 1). On the other hand but it continued for 120 minutes in K-EDTA anticoagulant and then stopped. Platelet count decreased significantly $(P=0.0001)$ in the presence of Na-citrate in the same period (Table 1), but not in the presence of EDTA (Figure 2).

In the second part of the study, we determined MPV and the platelet count at 90 minutes after blood collection into K-EGTA and after 120 minutes of blood collection into $\mathrm{Na}$-citrate for 100 healthy volunteers. Markedly, we did not find a correlation between MPV and platelet count for this group of 100 subjects (Figure 3a, b). Normal ranges of MPV were 7.2-10.8 fL (95\% confidence interval) in the presence of EDTA and 6.1-9.5 fL (95\% confidence interval) in the presence of citrate (Figure 4). Again, as expected, MPV was lower with Na-citrate than with K-EDTA anticoagulant. 


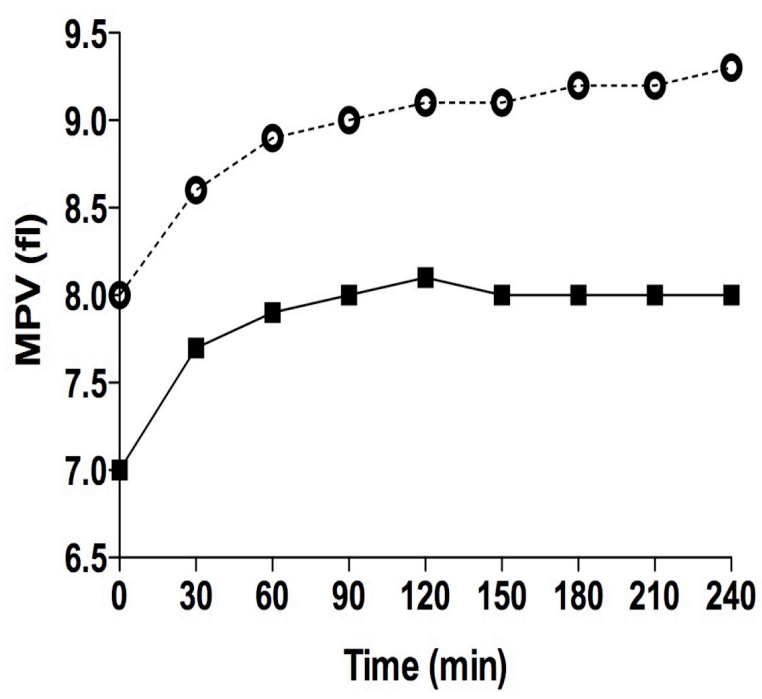

Figure 1: MPV measured in blood anticoagulated with K-EDTA (circles) or Na-citrate (squares) function of time after blood drawing. Mean $(n=20)$.

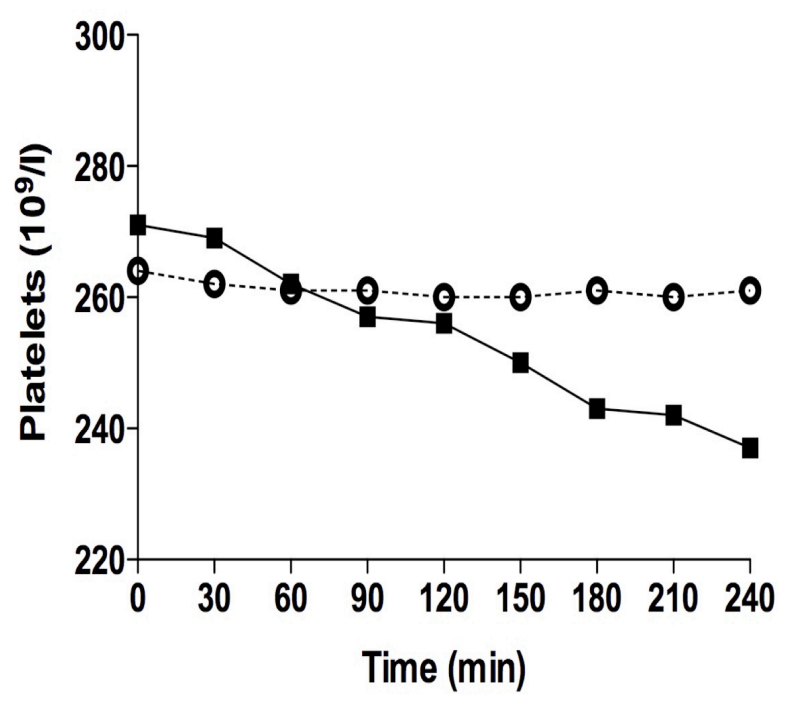

Figure 2: Platelet count $\left(10^{9} / \mathrm{L}\right)$ in blood in KEDTA (circles) or Na-citrate (squares) as a as a function of time after blood drawing. Mean $(n=20)$.

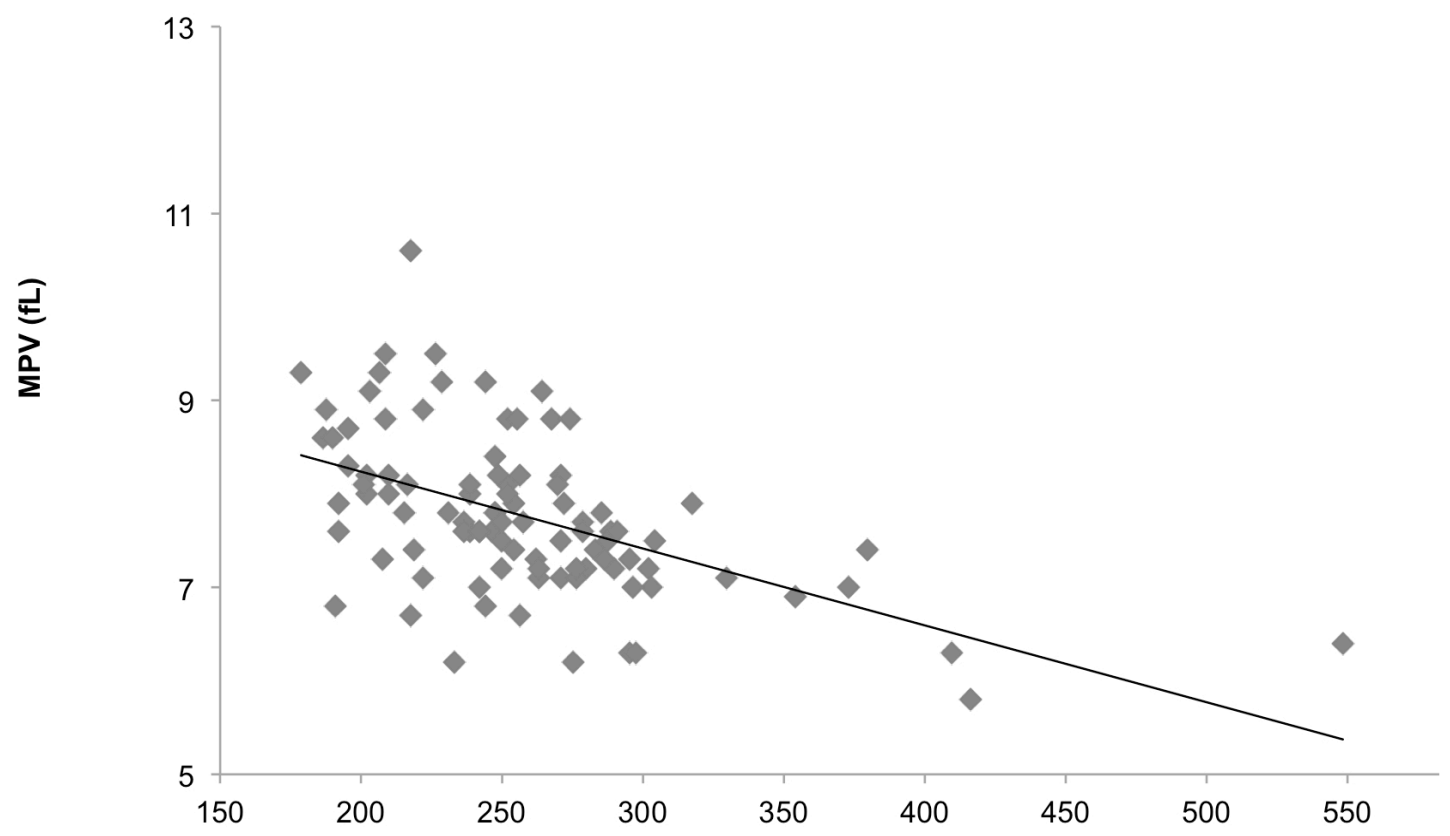

Platelet count $\left(\times 10^{9 / L}\right)$

Figure 3a: Correlation of MPV (fL) with platelet count $\left(x 10^{9} / \mathrm{L}\right)$ of blood samples collected into Nacitrate after 90 minutes $\left(n=100, R^{2}=0.28\right.$, Pearson correlation -0.53$)$. 


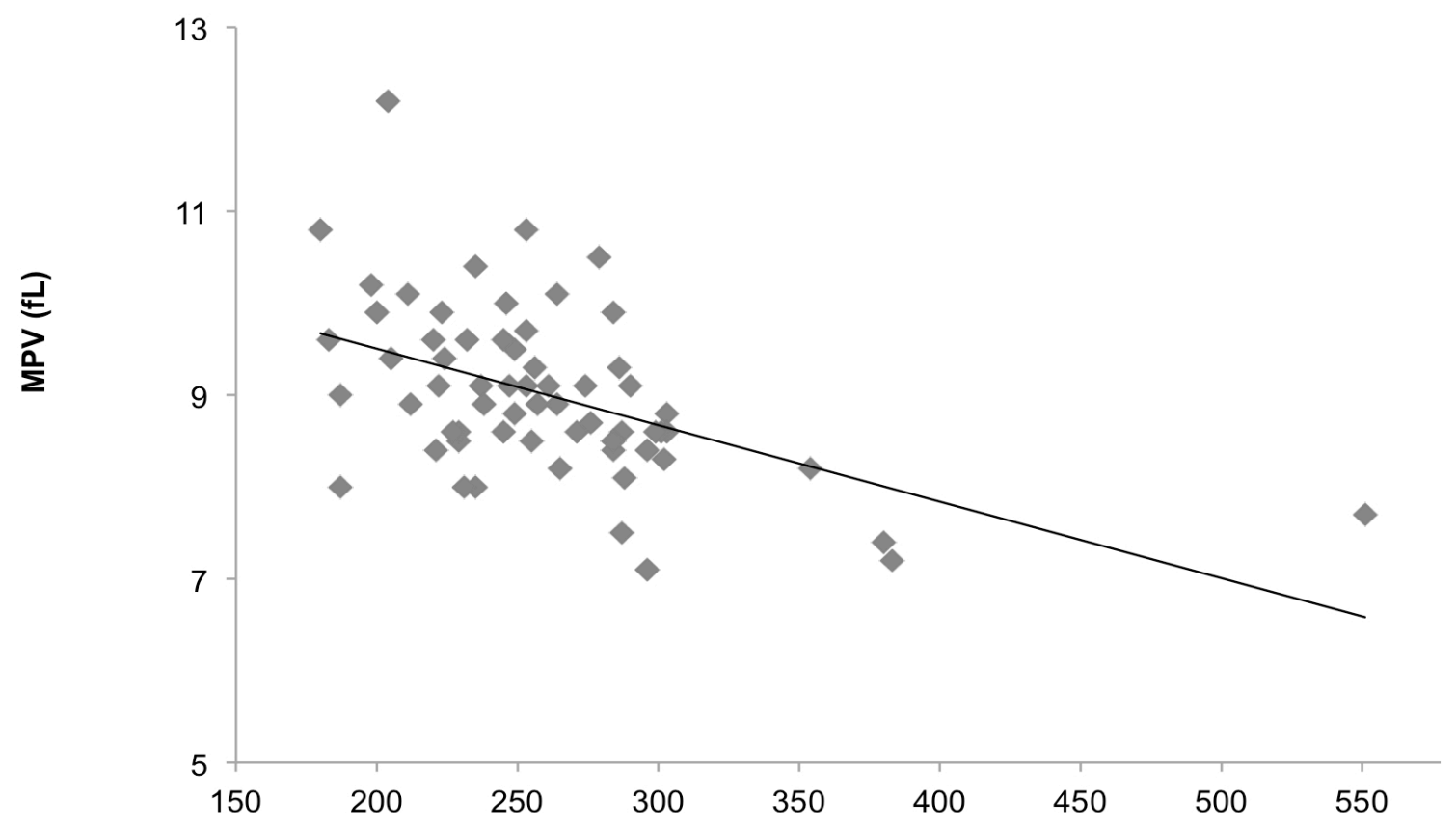

Platelet count (x109/L)

Figure 3b: Correlation of MPV (fL) with platelet count $\left(\times 10^{9} / \mathrm{L}\right)$ of blood samples collected into K-EDTA after 120 minutes $\left(n=100, R^{2}=0.22\right.$, Pearson correlation -0.47$)$.

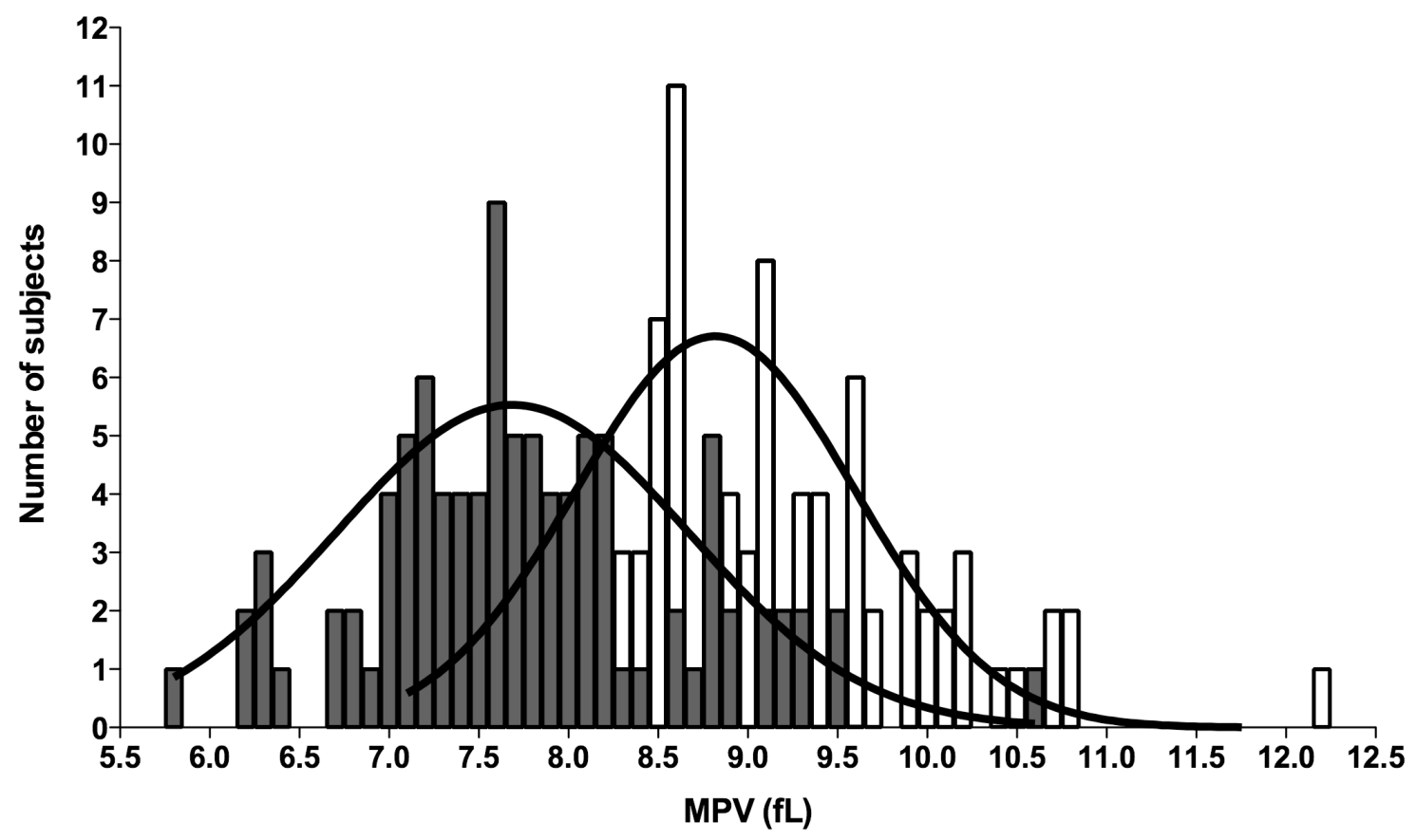

Figure 4: Normal ranges of MPV (fL) in blood anticoagulated with K-EDTA (white bars) or Na-citrate (grey bars), measured at 120 and 90 minutes after blood drawing, respectively $(n=100)$. 


\section{Discussion}

In this paper we determined reference values for the MPV using two different anticoagulants after standard time intervals of blood drawing using a Coulter LH-750 Hematology Analyzer. The time points after which maximal values of the MPV were reached was reached were after 120 minutes in K-EDTA anticoagulant and after 60 minutes in Na-citrate anticoagulant. To our knowledge this is a first report on such optimation of MPV measuruments.

Several authors have reported on variation of the MPV measurement with type of anticoagulation and time of measurement. Thompson et al. concluded that the MPV reaches a stable value after 2 hours of blood taking, independently of the anticoagulant, although most consistent results were obtained with acid-citratedextrose or Na-EDTA [3]. In our study, we found a plateau level for the MPV after 120 minutes in K-EDTA anticoagulant and after 60 minutes in Na-citrate. Bath and co-workers used EDTA and two concentrations of Na-citrate (low 9:1 or high 4:1), and reported that MPV at high Na-citrate minimally changed until 90 minutes [2]. In our study, MPV in Na-citrate samples reached a maximal level after 60 minutes. This may be an effect of the different citrate concentration: $3.2 \%$ in our case and $3.8 \%$ in case of Bath and co-workers. Recently, Dasterdi et al. found that the MPV in citrate was smaller than in EDTA media, but that these values yet correlated when blood samples from different subjects were compared. The authors concluded that both methods might be used clinically within 1 hour of blood taking, but did not consider time-dependency of the MPV measurement [10].

In another study from Macey et al., platelet count did not change significantly over time in EDTA anticoagulant [18]. Furthermore, there was no significant difference between EDTA, citrate and dipyridamole media, when samples were stored for a period of 180 minutes. The latter is in contrast to our results. We found that, in citrate, platelet count decreased significantly over a period of 240 minutes. Thompson and co-workers describe a stable platelet count over an interval of 8 hours for samples stored in EDTA anticoagulant, but they observed a relevant decrease from 102.4 to $94.1 \times 10^{9} / \mathrm{L}$, when samples were preserved in citrate. The authors argue that in citrate anticoagulant the $\mathrm{pH}$ may increase in time, which leads to platelet stimulation [3].

In our study, we measured in EDTA anticoagulant a stable mean count of 264 $\times 10^{9} / \mathrm{L}$ platelets $( \pm 44.4 \mathrm{SD}$ ), while in Na-citrate the mean platelet count decreased 
significantly from $271 \pm 43.7$ to $237 \pm 36.5 \times 10^{9} / \mathrm{L}$ platelets. This is in accordance with data from Dasterdi and co-workers, who found an about $36 \%$ lower platelet count in citrate than in EDTA anticoagulant medium [10].

In the second part of the study, where data from 100 healthy volunteers were analyzed, we did not find a reciprocal relationship between platelet count and MPV. This contrasts to the findings of other authors. One reason for this may be that, for instance, Bath and co-workers compared platelet count measured in EDTA with MPV measured in Na-citrate [2]. These authors have also measured MPV only at a single time point. Furthermore, Thompson and co-workers [3] analysed blood samples from only 52 volunteers, instead of the 100 samples measured in the present study. Also Dasterdi et al. measured samples from only 61 patients, who where referred to the hospital for hematologic diagnostics [10]. All these authors concluded that there is an inverse relation between MPV and platelet count [2, 3, 10]. In our view, the absence of a reciprocal relation between platelet count and their size is because we performed the MPV measurement at an optimized time, and unsed a larger population of healthy volunteers. The latter might be seen as a disadvantage, because in clinical practice we do not take blood samples in healthy subjects.

We conclude that the choice of anticoagulant (standard K-EDTA or Na-citrate) resulted in different MPV values using the Coulter Analyzer. For each anticoagulant medium, we recommend to wait until a stable value of MPV due to platelet swelling has been reached. This was after 120 minutes in K-EDTA and after 60 minutes in Na-citrate anticoagulant. Furthermore, we did not find an inverse relationship between MPV and platelet count. For further standardization, it is recommended to determine time-dependent reference values. Standardization of the MPV measurements will be necessary to elucidate a relation of the MPV and platelet activity. 


\section{References}

$1 \quad$ Giles C: The platelet count and mean platelet volume. Br J Haematol. 1981;48: 31-7.

2 Bath PM: The routine measurement of platelet size using sodium citrate alone as the anticoagulant. Thromb Haemost. 1993;70: 687-90.

3 Thompson CB, Diaz DD, Quinn PG, Lapins M, Kurtz SR, Valeri CR: The role of anticoagulation in the measurement of platelet volumes. Am J Clin Pathol. 1983;80: 327-32.

$4 \quad$ Trowbridge EA, Reardon DM, Hutchinson D, Pickering C: The routine measurement of platelet volume: a comparison of light-scattering and aperture-impedance technologies. Clin Phys Physiol Meas. 1985;6: 221-38.

5 Martin JF, Trowbridge EA, Salmon G, Plumb J: The biological significance of platelet volume: its relationship to bleeding time, platelet thromboxane B 2 production and megakaryocyte nuclear DNA concentration. Thromb Res. 1983;32: 443-60.

6 Jakubowski JA, Thompson CB, Vaillancourt R, Valeri CR, Deykin D: Arachidonic acid metabolism by platelets of differing size. $\mathrm{Br} \mathrm{J}$ Haematol. 1983;53: 503-11.

7 Giles H, Smith RE, Martin JF: Platelet glycoprotein Ilb-IIla and size are increased in acute myocardial infarction. Eur J Clin Invest. 1994;24: 69-72.

$8 \quad$ Smith NM, Pathansali R, Bath PM: Platelets and stroke. Vasc Med. 1999;4: 165-72.

9 Bessman JD, Williams LJ, Gilmer PR, Jr.: Mean platelet volume. The inverse relation of platelet size and count in normal subjects, and an artifact of other particles. Am J Clin Pathol. 1981;76: 289-93.

Dastjerdi MS, Emami T, Najafian A, Amini M: Mean platelet volume measurement, EDTA or citrate? Hematol. 2006;11: 317-9.

11 Thompson CB: Selective consumption of large platelets during massive bleeding. $\mathrm{Br}$ Med $\mathrm{J}$ (Clin Res Ed). 1985;291: 95-6.

12 Boldt J, Zickmann B, Benson M, Dapper F, Hempelmann G, Schindler E: Does platelet size correlate with function in patients undergoing cardiac surgery?. Intensive Care Med. 1993;19: 44-7.

13 Bozkurt N, Yilmaz E, Biri A, Taner Z, Himmetoglu O: The mean platelet volume in gestational diabetes. J Thromb Thrombolysis. 2006;22: 51-4.

14 Colkesen Y, Acil T, Abayli B, Yigit F, Katircibasi T, Kocum T, Demircan S, Sezgin A, Ozin B, Muderrisoglu $\mathrm{H}$ : Mean platelet volume is elevated during paroxysmal atrial fibrillation: a marker of increased platelet activation? Blood Coagul Fibrinolysis. 2008;19: 411-4.

Coban E, Ozdogan M, Yazicioglu G, Akcit F: The mean platelet volume in patients with obesity. Int J Clin Prac. 2005;59: 981-2.

16 D'Erasmo E, Aliberti G, Celi FS, Romagnoli E, Vecci E, Mazzuoli GF: Platelet count, mean platelet volume and their relation to prognosis in cerebral infarction. J Intern Med. 1990;227: $11-4$.

17 Demirtunc R, Duman D, Basar M, Bilgi M, Teomete M, Garip T: The relationship between glycemic control and platelet activity in type 2 diabetes mellitus. J Diabetes Complications. 2009;23: 89-94.

18 Macey M, Azam U, McCarthy D, Webb L, Chapman ES, Okrongly D, Zelmanovic D, Newland A: Evaluation of the anticoagulants EDTA and citrate, theophylline, adenosine, and dipyridamole (CTAD) for assessing platelet activation on the ADVIA 120 hematology system. Clin Chem. 2002;48: 891-9. 


\section{Chapter 3}

Do blood collection methods influence whole-blood platelet function analysis?

Marcus D. Lancé, Yvonne M.C. Henskens, Patty Nelemans, Maurice H.S. Theunissen, René van Oerle, Henri M. Spronk, Marco A.E. Marcus 


\section{Abstract}

Background: Pre-analytical variables of blood samples used for platelet function measurements can be influenced by residual coagulation. How these variables affect platelet function analysis is not completely known. In this paper, we compared how the site of blood collection and the type of blood puncture affect whole-blood platelet function, as determined by multiple electrode aggregometry (MEA) and the platelet function analyser 100 (PFA-100). In addition, we determined whether contact activation of the coagulation system affected the whole-blood measurements.

Methods: In Part 1, 11 patients scheduled for elective cardiac surgery gave blood from three lines (venous, arterial, central venous) and by venipuncture. MEA and PFA-100 were performed, blinded for the type of blood sample. In part 2, 20 patients gave blood from the arterial line. Samples were collected with a syringe or ditectly into vacuum tubes, containing citrate with or without corn trypsin inhibitor. Plasma was prepared to measure thrombin generation.

MEA was measured in response to ADP, arachidonic acid, collagen, ristocetin, or thrombin receptor-activating peptide. PFA-100 was used with cartriges containing collagen/epinephrine or collagen/ADP. Thrombin generation was assessed in platelet-poor plasma with $1 \mathrm{pM}$ tissue factor and $4 \mu \mathrm{M}$ phospholipids or without trigger. Data were analysed using a two-way mixed-effects model for intraclass correlations.

Results: In Part 1, the MEA and PFA-100 tests gave similar results for blood samples drawn from each of the four collection sites. In part 2, only the MEA test with arachidonic acid revealed significant differences between the two collection methods. Anticoagulation with corn trypsin inhibitor inhibited thrombin generation regardless of the blood collection method.

Conclusions: Blood sampling from all lines or venipuncture gave similar results for MEA and PFA-100 assays. Contact activation is always detectable, but does not appear to influence the outcome of platelet function tests. For platelet function analysis, blood can be collected from venous or arterial or by venipuncture. 


\section{Introduction}

Experimental differences in blood drawing, such as the vessel from which the blood sample is taken and the size of the needle used for blood collection, are considered to affect plasmatic coagulation. The effects of these pre-analytical variables on standard coagulation measurements, such as the activated partial thromboplastin time (aPTT) and the prothrombin time (PT) are however negligible or mild [1]. How such differences in blood drawing affect platelet function has not been examined. Variation here might explain part of the known overall variability in platelet function analysis [2]. Currently, some methods are available to study platelet function in whole blood, in particular multiple electrode aggregometry (MEA) and the Platelet Function Analyzer-100 (PFA-100). These tests have been shown to be useful in the monitoring of patient treatment with platelet aggregation inhibitors, e.g. in interventional therapy upon coronary artery disease [3, 4]. The same whole-blood platelet function tests can help to guide anti-platelet therapy in (surgical) patients, who have a bleeding risk. In these settings, they have been introduced in diagnostic algorithms [5-8].

In general, blood collection methods are not standardized for platelet function analysis. Most medical centres use vacuum systems for blood collection in the outpatient clinics, but operating theatres, emergency departments and intensive care departments may collect blood primarily from a line with a syringe and then transfer it to a vacuum tube. The diameter and the length of the puncture system may lead to contact activation of the coagulation system, while the shear stress in the line may influence platelet function [9-12]. Moreover, blood samples are often collected from various indwelling lines for patient convenience and ease of access [7, 13]. In this case, differences in oxygen content between venous and arterial blood samples may influence both the coagulation and platelet functions, e.g. due to the higher viscosity of oxygenated blood [14, 15].

Because of the presumed sensitivity of whole-blood platelet function tests for such pre-analytic variables, we hypothesized that the needle bore size, the diameter and length of blood line, and the origin of the blood (arterial, venous or central venous) influence the test results. The aim of the present study was to compare the effects of different blood collection methods from four sampling on sites on two whole-blood platelet function tests, MEA and PFA-100. In addition, we aimed to determine whether the procedure of blood collection influenced the extent of contact activation in the different blood samples. 


\section{Material and methods}

\section{Patients and blood drawing}

The studies were approved by the local medical ethics board. After written informed consent, in total 31 patients were included, scheduled for elective cardiac surgery at Maastricht University Medical Centre, within a period of one month. Patients were included, who required any type of cardiac surgical procedure (CABG, on/off-pump, valve operations, and rhythm surgery), and needed vascular access with an arterial line, a venous line and a large-bore central venous line. Excluded were patients, who were on anticoagulant medication (e.g., LMWH, vitamin $\mathrm{K}$ antagonists) and/or platelet inhibitors at least 5 days before operation.

Patients arriving in the operating theater underwent standard monitoring procedures, i.e. ECG, plethysmographic oxymetry and non-invasive blood pressure. Subsequently, a venous line (Vasofix Safety, 16G, $1.7 \times 50 \mathrm{~mm}$, Braun Melsungen, Germany) and an arterial line (Radial Artery Catheterization set, 20G, $1.1 \times 44.5 \mathrm{~mm}$ Arrow International, Reading, Pennsylvania, USA) were placed. General anaesthesia was induced, following institutional guidelines. After intubation, a large-bore central venous line (Arrow-Flex polyurethane sheath 8.5 Fr., $2.97 \times 100$ mm, Arrow International) was placed in the right internal jugular vein. Finally, a venipuncture (G18 needle, $1.3 \times 40 \mathrm{~mm}$, Terumo Medical, Europe) was performed without use of a tourniquet.

Blood samples were collected immediately after drawing and before start of the operation. All samples were transported to the laboratory by walking. Haemoglobin and platelet count were measured to check for abnormal values using a Beckman Coulter LH-750 analyser (Beckman Coulter, Woerden, the Netherlands).

Platelet function was analysed by whole-blood MEA (Multiplate analyser, Dynabyte, Munich, Germany) using the following commercially available agonists in compliance with the manufacturer's recommendations: adenosine diphosphate (ADP, $6.4 \mu \mathrm{M}$ ), arachidonic acid (ASPI, $0.5 \mathrm{mM}$ ), collagen (Col, $3.2 \mu \mathrm{g} / \mathrm{mL}$ ), ristocetin (Risto, $0.2 \mathrm{mg} / \mathrm{mL}$ ), and thrombin receptor-activating peptide (TRAP, $32 \mu \mathrm{M}$ ). The procedures have been published elsewhere [16]. In part of the blood samples, platelet activity was measured with the PFA-100 (Siemens Medical Solutions Europe), using cartridges with collagen/epinephrine and collagen/ADP according to the manufacturer's recommendations. All whole-blood tests were performed at $37^{\circ} \mathrm{C}$ 
by the same technician, within 90 minutes after sampling. The Multiplate analyser and PFA-100 device were calibrated according to the manufacturer's instructions, and a daily quality check was done.

\section{Part 1}

In Part 1 of the study, blood was collected from 11 patients in four different ways in the following sequence: from the venous line, the arterial line, central venous line, and then by venipuncture. All blood samples were collected with a $10 \mathrm{~mL}$ syringe, after discarding the first $10 \mathrm{~mL}$. Subsequently, three sample tubes with different anticoagulants were filled, and the blood from the syringe was gently mixed. The following anticoagulant tubes were used: EDTA (K-EDTA, BD Vacutainer, Plymouth, UK), citrate (Na-citrate $0.105 \mathrm{M}, 3.2 \%$, BD Vacutainer), hirudin ( $3 \mathrm{~mL}, 15 \mu \mathrm{g} / \mathrm{mL}$, Dynabyte, Munich, Germany). The sampling tubes were colour-coded in a way that the laboratory technician was blinded for the collection site.

\section{Part 2}

In Part 2 of the study, arterial blood was collected from 20 patients via an arterial line before the induction of anaesthesia. Investigated was whether contact activation was induced by blood collection into vacuum tubes or via a syringe. Platelet function again was assessed by MEA and PFA-100, while thrombin generation was measured to check for contact activation.

After discarding the first $10 \mathrm{~mL}$ of blood, two sets of blood samples were collected: EDTA tubes (K-EDTA, BD Vacutainer), citrate tubes $(0.105 \mathrm{M}, 3.2 \%$, BD Vacutainer), and hirudin tubes ( $3 \mathrm{~mL}, 15 \mu \mathrm{g} / \mathrm{mL}$, hirudin, Dynabyte). In addition, vacuum tubes containing corn trypsin inhibitor (CTI, $40 \mu \mathrm{g} / \mathrm{mL}$, Haematologic Technologies, Essex, VT, USA) were used for thrombin generation testing. A first four samples were directly collected into vacuum tubes (EDTA, citrate, hirudin, CTI). A second set of samples was collected via a $10 \mathrm{~mL}$ syringe and then transferred to the vacuum tubes. For thrombin generation measurements, relevant blood samples were processed to platelet-poor plasma (PPP), and stored at $-80^{\circ} \mathrm{C}$ until use.

Thrombin generation was analysed in PPP by means of the calibrated automated thrombogram (CAT, Thrombinoscope, Maastricht, the Netherlands) using three reagent modes: the first without tissue factor/phospholipids; the second with 
only $4 \mu \mathrm{M}$ phospholipid, and the third with $1 \mathrm{pM}$ tissue factor combined with $4 \mu \mathrm{M}$ phospholipids (PPlow reagent, Thrombinoscope, Maastricht, the Netherlands).

Platelet function analysis was performed by MEA and by using the PFA-100, as described for Part 1. Considering that a $25 \%$ difference for MEA suggests clinical relevance, $n=10$ was necessary to reach a power of $80 \%$ with an alpha error of 0.05 .

\section{Statistics}

Platelet function tests were compared using the intraclass correlation coefficient (ICC). A two-way mixed effects model was used, in which ICC values $>0.5$ define a good agreement, and $>0.7$ define a strong correlation. The results of Part 2 of the study were analysed using the Mann-Whitney $U$ test. $P$ values $<0.05$ were considered statistically significant. For statistical analyses, we used the program SPSS 15 for Windows (SPSS, Chicago, IL, USA).

\section{Results}

In Part 1 of the study, 11 patients were included, resulting in 44 blood samples. Demographic data of the patients are shown in Table 1. One sample originating from

Table 1: Demographic data of patients participating in the studies part $1(n=11)$ and part $2(n=20)$.

\begin{tabular}{lllll}
\hline & \multicolumn{3}{l}{ Part 1 (n = 11) } & \multicolumn{3}{l}{ (n = 20) } \\
\cline { 2 - 5 } Age (years) & 70.1 & $(8.37)$ & 64.3 & $(11.82)$ \\
Female (n) & 4 & $(36 \%)$ & 9 & $(45 \%)$ \\
Weight (kg) & 80.5 & $(16.43)$ & 79.7 & $(18.56)$ \\
Body mass index (kg/m $\left.{ }^{2}\right)$ & 27.2 & $(5.99)$ & 26.5 & $(6.01)$ \\
Arterial hypertension (n) & 6 & $(54.6 \%)$ & 14 & $(70 \%)$ \\
Diabetes mellitus (n) & 2 & $(18.2 \%)$ & 7 & $(35 \%)$ \\
Peripheral arterial disease (n) & 4 & $(36.4 \%)$ & 5 & $(20 \%)$ \\
COPD (n) & 3 & $(27.3 \%)$ & 4 & $(20 \%)$ \\
Hypercholesterolemia (n) & 3 & $(27.3 \%)$ & 6 & $(30 \%)$ \\
Hb (mM) & 7.73 & $(0.94)$ & 7.94 & $(1.41)$ \\
Hct (\%) & 0.37 & $(0.04)$ & 0.38 & $(0.06)$ \\
Platelets (10 $/ \mathbf{L})$ & 230 & $(36.63)$ & 209.9 & $(34.90)$ \\
MPV (fL) & 9.11 & $(1.2)$ & ND & ND \\
\hline
\end{tabular}

Data are mean (SD, absolute or as percentage), $\mathrm{COPD}=$ chronic obstructive pulmonary disease, $\mathrm{ND}=$ not determined. 

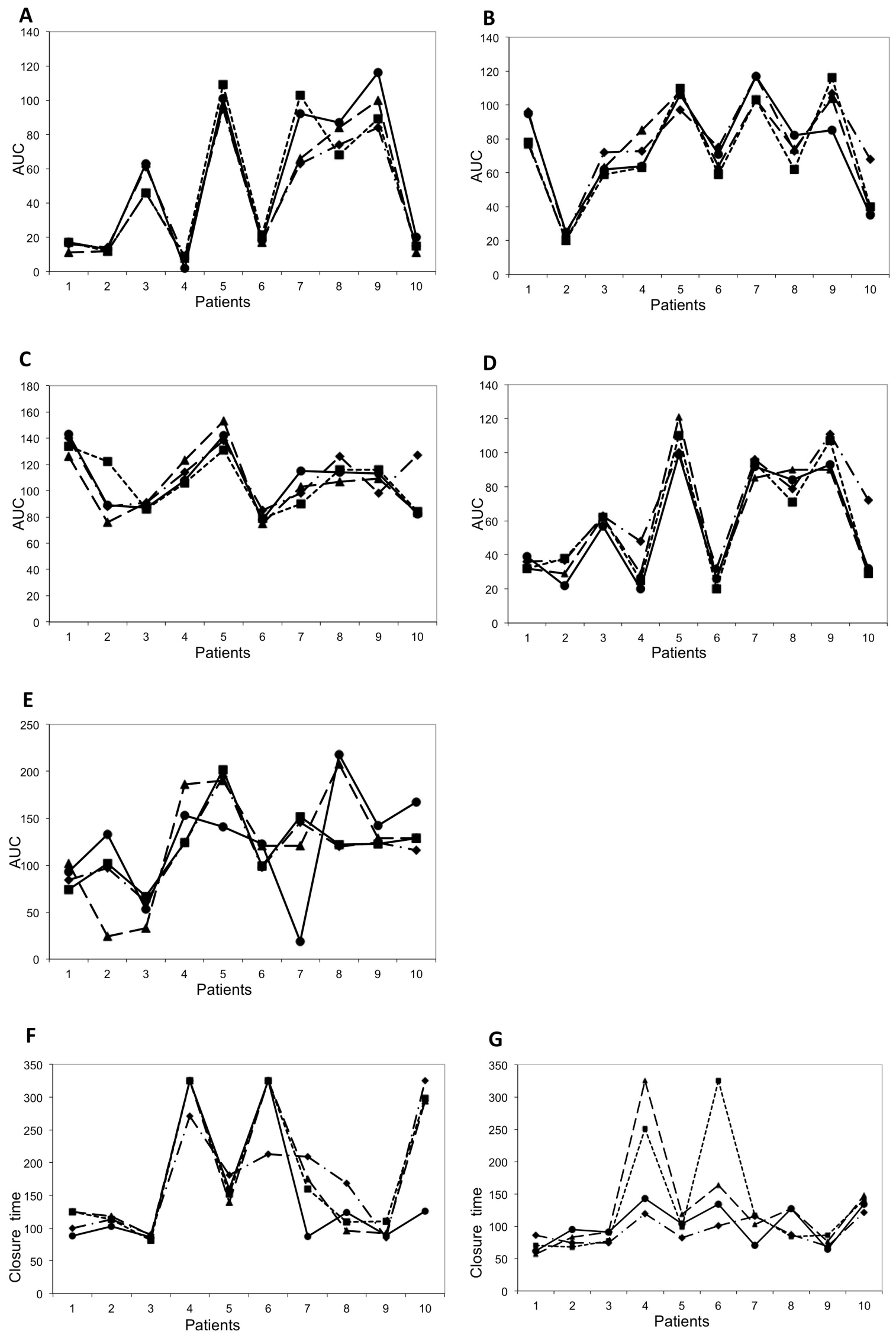

Figure 1: Part 1 of the study. Whole-blood platelet function tests were performed using blood 
samples from 10 patients, that were collected from four different sites: venous line $(\mathbf{\Lambda})$, arterial line $(\bullet)$, central venous line $(\bullet)$, or by venipuncture $(\boldsymbol{\square})$. (A-D) Results for MEA are given as area-under the curve (AUC, relative values). (E-F) Results for PFA-100 are given as closure time (seconds). Separately established reference ranges for the different tests are for MEA (AUC): ASPI test 51-119 (Panel A), ADP test 33-108 (Panel B), TRAP test 70-130 (Panel C), Col test 45-125 (Panel D), RISTO test 90-201 (Panel E). Reference ranges for PFA-100 are: Col/EPI cartridge 67-171 seconds (Panel F), Col/ADP cartridge 55-119 seconds (Panel G). Intraclass correlation coefficients (ICC) were: ASPI test 0.95 (Panel A), ADP test 0.90 (Panel B), TRAP test 0.73 (Panel C), 0.91 Col (Panel D), 0.51 RISTO (Panel E). For the PFA-100 tests, ICC values were $0.81 \mathrm{Col} / \mathrm{EPI}$ (Panel F), and 0.46 PFACol/ADP (Panel $G)$. ( $n=10$, exclusion of one patient)

a venipuncture was coagulated before analysis, making it ineligible for processing. Analysis was hence done on the remaining 43 blood samples.

The ICC for interchangeability of the type of blood sample pointed to strong correlations for various platelet function tests (Figure 1). Regarding MEA, ICC values were 0.95 for the ASPI test, 0.90 for the ADP test, 0.91 for the Col test, and 0.73 for the TRAP test. Regarding PFA-100, the ICC value was 0.81 for Col/EPI cartridges. A good correlation was found for MEA using the RISTO test (ICC =0.51), and a moderate correlation for PFA-100 with Col/ADP cartridges (ICC $=0.46)$.

In Part 2 of the study, 40 sets of 2 blood samples from 20 patients were collected by two collection methods, each using two sample tubes (citrate and citrate combined with CTI). Demographic details of these 20 patients are presented in Table 1. The results from one sample had to be excluded due to clotting in the tube. Mean platelet count did not differ between the two collection methods (Table 2).

Table 2: Part 2 of the study. Comparison of blood collection with a syringe versus a vacuum system.

\begin{tabular}{|c|c|c|c|c|c|c|c|}
\hline & \multirow[b]{2}{*}{ PIt count } & \multicolumn{4}{|c|}{ MEA (AUC) } & \multicolumn{2}{|c|}{ PFA-100 (s) } \\
\hline & & ASPI & ADP & Coll & TRAP & Coll/Epi & Coll/ADP \\
\hline Syringe & $\begin{array}{l}209.9 \\
(34.90)\end{array}$ & $\begin{array}{l}26.21 \\
(22.53)\end{array}$ & $\begin{array}{l}53.21 \\
(19.23)\end{array}$ & $\begin{array}{l}54.37 \\
(20.40)\end{array}$ & $\begin{array}{l}86.00 \\
(26.92)\end{array}$ & $\begin{array}{l}163.6 \\
(60.45)\end{array}$ & $\begin{array}{l}106.0 \\
(49.74)\end{array}$ \\
\hline $\begin{array}{c}\text { Vacutain } \\
\text { er }\end{array}$ & $\begin{array}{l}208.4 \\
(45.79)\end{array}$ & $\begin{array}{l}31.58 \\
(25.14)\end{array}$ & $\begin{array}{l}57.11 \\
(21.71)\end{array}$ & $\begin{array}{l}56.74 \\
(21.01)\end{array}$ & $\begin{array}{l}88.42 \\
(17.47)\end{array}$ & $\begin{array}{l}160.5 \\
(69.51)\end{array}$ & $\begin{array}{l}93.21 \\
(38.57)\end{array}$ \\
\hline P-value & 0.94 & $0.03^{*}$ & 0.87 & 0.70 & 0.86 & 0.82 & 0.46 \\
\hline
\end{tabular}

Platelet count is in $10^{9}$ platelets/L. MEA results with various agonists are presented as area under the curve (AUC). PFA-100 results are presented in seconds. Blood was collected in to citrate. Reference range for the various tests are, as described for Figure 1. Only the ASPI assay was different between the two ways of blood drawing $\left({ }^{*} P=0.03\right)$. Data presented as means $\pm \operatorname{SD}(\mathrm{n}=20)$. 
In PPP preparations derived from these blood samples collected in the prsence of CTI, thrombin was measured in order to determine the extent of contact activation. In Figure 2, it is shown that blood collection in citrate/CTI inhibited thrombin generation significantly more effectively than in citrate alone. This was apparent from a prolonged lag-time to thrombin generation and a lower thrombin peak height. In the absence of tissue factor, the lag time is highly prolonged and no peak develops in the citrate/CTI samples (Flgure 2A-D). With tissue factor present, citrate/CTI samples did develop a thrombin peak, but this was still significantly lower than in the citrate samples (Figure 2E-F). This figure furthermore shows that essentially the same thrombin generation results were obtained, when blood was drawn via the two collection methods (syringe versus vacuum blood collection). This suggests that the amount of contact activation (i.e. the CTI-inhibitable part of the thrombin generation) was independent of the method of blood collection.

In MEA, there was little difference in platelet aggregation between the two types of blood collection (Table 2). Only in the ASPI test, platelet aggregation was lower in syringe blood samples than in blood samples directly collected in vacuum tubes (syringe 26.2 vs. vacuum 31.6 AUC, $P=0.03$ ). However, the ASPI test gave significantly lower values than the manufacturer's reference ranges. Other values were not different with respect to the blood collection method. In the PFA-100 test, clotting time with either Col/ADP or Col/EPI cartridges did not reveal significantly different values between the two blood collection techniques (Table 2).

\section{Discussion}

In Part 1, we used the Multiplate analyser, an automated impedance aggregometer, and the PFA-100 analyser to determine platelet function in whole blood. No relevant differences were found in platelet reactivity between blood samples, drawn from four different puncture sites. Also the PFA-100 analyser did not reveal differences between the four sites of blood sampling, when using the Col/EPI cartridge. However, the PFA-100 device with Col/ADP cartridges showed only a moderate intraclass correlation. These results are in line with those from Mani and colleagues, who compared two different blood collection methods. The authors report similar PFA-100 measurements for blood samples drawn by venipuncture with a $21 \mathrm{G}$ needle and venipuncture with a $21 \mathrm{G}$ winged collecting system (also known as butterfly system). Moreover, they show that the type of blood collection also does not influence platelet 

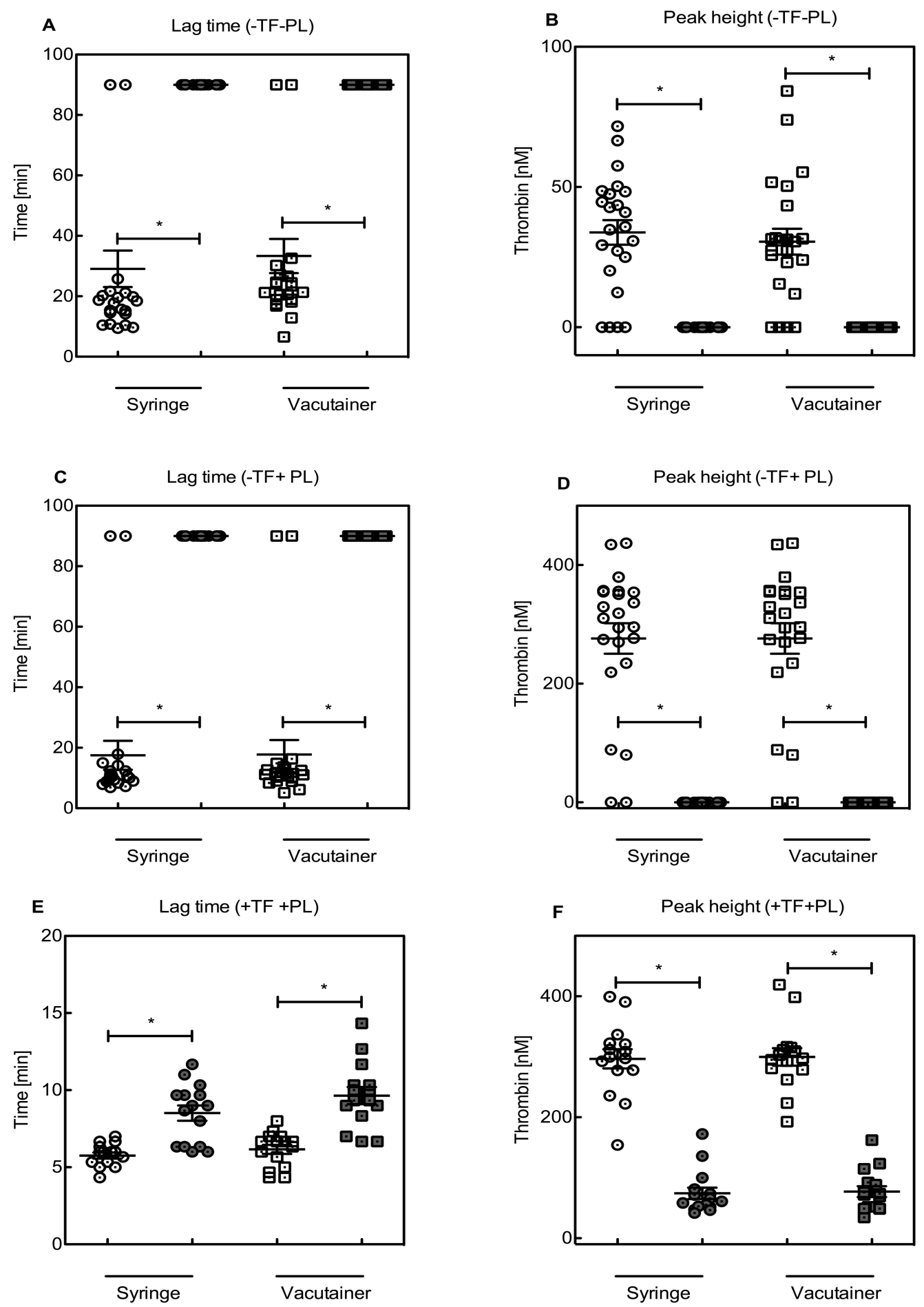

Figure 2: Part 2 of the study. Results of thrombin generation measurement of PPP from blood samples collected with a syringe or directly into a vacutainer. Blood samples were anticoagulated with citrate alone (open symbols) or with citrate/CTI (closed symbols). The PPP was activated with $\mathrm{CaCl}_{2}$ in the presence of different triggers, as indicated. (A, B) Lag-time to thrombin generation and thrombin peak height in the absence of tissue factor or phospholipids ( $P L)$. (C, D) Lag-time to thrombin generation and thrombin peak height with $4 \mu \mathrm{M} P L$. (E, F) Lag-time to thrombin generation and thrombin peak height with1 pM tissue factor and $4 \mu \mathrm{M} P \mathrm{PL} . \mathrm{N}=20,{ }^{*} \mathrm{P}<0.05$. 
function as assessed by light transmission aggregometry [17].

In a study with eight elective cardiac surgical patients, Rubens et al. investigated blood samples collected from an arterial line, a central venous line and a single venipuncture by flow cytometry. The authors reported different degrees of platelet activation markers (factor XIIla, thrombospondin, activated glycoprotein $\mathrm{Ilb} / \mathrm{llla}$ and P-selectin) dependent on the collection site. The arterial samples showed more signs of activation. They attribute their findings to different shear rates in the lines and to more contact activation due to length of the catheters. The authors recommended to take venous blood for platelet function analysis, regardless of whether it is from a central venous line or from venipuncture [18]. The present results, using whole-blood platelet function tests, are not in line with this. A possible explanation is that the whole-blood platelet function tests, which we applied, are less sensitive to pre-analytical variation than the flow cytometry method.

Another reason for the different results between arterial and venous samples might be shear stress and oxygen content of the samples. Unfortunately, there are no investigations on this topic with regard to platelet function tests. In a study by Frumento et al, this item seemed to be relevant for fibrin formation [19]. The authors examined blood samples from an arterial line, a central venous line and a pulmonary artery catheter using thromboelastography, but did not focus on platelet function tests. They demonstrated significant differences in nearly all thromboelastography parameters with respect to the site of blood collection. Differences were smaller between samples from the arterial and central venous lines that between samples from the central venous and pulmonary arterial lines. The main conclusion was that the oxygen content does not play a role, but that shear stress may be a more crucial factor [19]. This is in not in agreement with the conclusions of Manspeizer and colleagues, who proposed that the difference in oxygen content explained their differences in thromboelastography, when comparing arterial with central venous blood samples.

In Part 2, the inhibitory effect of blood collection into CTI media regarding thrombin generation pointed to the presence of contact activation in the various blood samples, which might contribute to platelet activation. We hence conclude that the choice whether to use a direct blood collection technique (vacuum system) or an indirect technique (syringe) does not influence the residual coagulation in the blood samples. Concerning the performed whole-blood platelet function tests (MEA and 
PFA-100), there was only a difference when arachidonic acid was used as activator. Because the values with both blood collection methods were below the reference range, we tend to conclude that blood from some of the patients had residual effects of acetylsalicylic acid, in spite of the fact that the drug was stopped three days before the intervention. The cessation period likely was too short to restore platelet function completely [22]. In a recent investigation of Dyszkiewicz-Korpanty, the authors showed a difference in light transmission aggregation when patients were on acetylsalicylic acid treatment and samples were agitated by pneumatic tube transport [23]. The relevance of this result is not clear, because both of our results are below the normal range.

Our study has clearly limitations. The sample size was calculated by power analysis, but may have been too small for strong conclusions. Not included were patients who suffer from coronary artery disease and therefore need treatment with platelet inhibitors. However, we did include cardiovascular patients, who received the same type of blood lines standardly. The time frame of the measurements may not have been optmal, i.e. analysing the blood samples within 90 minutes, as this was recommended by the manufacturer.

Taken together, our results justify that blood can be collected from each of the venous or arterial lines for reliable analysis of whole-blood platelet function using MEA and PFA-100 assays. Contact activation is always present in these blood samples, but apparently this does not influence platelet function test outcomes. Even though the way of blood collection does not seem to be relevant, it is still recommended to perform this in a standardized way. 


\section{References}

1 Lippi G, Salvagno GL, Montagnana M, Poli G, Guidi GC: Influence of the needle bore size on platelet count and routine coagulation testing. Blood Coagul Fibrinolysis. 2006;17: 557-61.

2 Cattaneo M, Hayward CP, Moffat KA, Pugliano MT, Liu Y, Michelson AD: Results of a worldwide survey on the assessment of platelet function by light transmission aggregometry: a report from the platelet physiology subcommittee of the SSC of the ISTH. J Thromb Haemost. 2009;7: 1029.

3 Siller-Matula JM, Christ G, Lang IM, Delle-Karth G, Huber K, Jilma B: Multiple electrode aggregometry predicts stent thrombosis better than the vasodilator-stimulated phosphoprotein phosphorylation assay. J Thromb Haemost. 2010;8: 351-9.

4 Hofer CK, Zollinger A, Ganter MT: Perioperative assessment of platelet function in patients under antiplatelet therapy. Expert Rev Med D. 2010;7: 625-37.

Reece MJ, Klein AA, Salviz EA, Hastings A, Ashworth A, Freeman C, Luddington RJ, Nair S, Besser MW: Near-patient platelet function testing in patients undergoing coronary artery surgery: a pilot study. Anaesthesia. 2011;66: 97-103.

6 Robier C, Neubauer M, Sternad H, Quehenberger F, Rainer F, Neumeister P: Evaluation of platelet function and pharmacological platelet inhibition in patients with myeloproliferative disorders using multiple electrode aggregometry. Thromb Res. 2010;126: 232-7.

7 Rahe-Meyer N, Winterhalter M, Boden A, Froemke C, Piepenbrock S, Calatzis A, Solomon C: Platelet concentrates transfusion in cardiac surgery and platelet function assessment by multiple electrode aggregometry. Acta Anaesthesiol Scand. 2009;53: 168-75.

8 Gorlinger K, Dirkmann D, Hanke AA, Kamler M, Kottenberg E, Thielmann M, Jakob H, Peters $\mathrm{J}$ : First-line therapy with coagulation factor concentrates combined with point-of-care coagulation testing is associated with decreased allogeneic blood transfusion in cardiovascular surgery: a retrospective, single-center cohort study. Anesthesiol. 2011;115: 1179-91.

9 Miyazaki Y, Nomura S, Miyake T, Kagawa H, Kitada C, Taniguchi H, Komiyama Y, Fujimura Y, Ikeda Y, Fukuhara S: High shear stress can initiate both platelet aggregation and shedding of procoagulant containing microparticles. Blood. 1996;88: 3456-64.

10 Breddin HK: Can platelet aggregometry be standardized? Platelets. 2005;16: 151-8.

11 Spronk HM, Dielis AW, De Smedt E, van Oerle R, Fens D, Prins MH, Hamulyak K, ten Cate H: Assessment of thrombin generation II: Validation of the calibrated automated thrombogram in platelet-poor plasma in a clinical laboratory. Thromb Haemost. 2008;100: 362-4.

12 Sommeijer DW, van Oerle R, Reitsma PH, Timmerman JJ, Meijers JC, Spronk HM, ten Cate $\mathrm{H}$ : Analysis of blood coagulation in mice: pre-analytical conditions and evaluation of a homemade assay for thrombin-antithrombin complexes. Thromb J. 2005;3: 12.

Solomon C, Hartmann J, Osthaus A, Schochl H, Raymondos K, Koppert W, Rahe-Meyer N: Platelet concentrates transfusion in cardiac surgery in relation to preoperative point-of-care assessment of platelet adhesion and aggregation. Platelets. 21: 221-8.

14 Schuurman PR, Albrecht KW: Intraoperative changes of transcranial Doppler velocity: relation to arterial oxygen content and whole-blood viscosity. Ultrasound Med Biol. 1999;25: 151-4.

Dellamonica J, Mazoyer E, Rosa JP, Cymbalista F, Samama C: Abrupt oxygen decrease influences thrombosis and bleeding in stenosed and endothelium-injured rabbit carotid arteries. Eur J Anaesthesiol. 2008;25: 1002-8.

Velik-Salchner C, Maier S, Innerhofer P, Streif W, Klingler A, Kolbitsch C, Fries D: Point-ofcare whole blood impedance aggregometry versus classical light transmission aggregometry for detecting aspirin and clopidogrel: the results of a pilot study. Anesth Analg. 2008;107: 1798-806.

17 Mani H, Kirchmayr K, Klaffling C, Schindewolf M, Luxembourg B, Linnemann B, Lindhoff-Last E: Influence of blood collection techniques on platelet function. Platelets. 2004;15: 315-8.

18 Rubens FD, Labow RS, Waghray G, Robblee J: The importance of sampling site in the measurement of whole-blood platelet flow cytometry. J Cardiothorac Vasc Anesth. 1998;12: 309-13.

19 Frumento RJ, Hirsh AL, Parides MK, Bennett-Guerrero E: Differences in arterial and venous thromboelastography parameters: potential roles of shear stress and oxygen content. J Cardiothorac Vasc Anesth. 2002;16: 551-4.

20 Spiess BD, Tuman KJ, McCarthy RJ, DeLaria GA, Schillo R, Ivankovich AD: Thromboelastography as an indicator of post-cardiopulmonary bypass coagulopathies. $\mathrm{J}$ Clin Monit. 1987;3: 25-30. 
21 Rahe-Meyer N, Winterhalter M, Hartmann J, Pattison A, Hecker H, Calatzis A, Solomon C: An evaluation of cyclooxygenase-1 inhibition before coronary artery surgery: aggregometry versus patient self-reporting. Anesth Analg. 2008;107: 1791-7.

22 Di Minno MN, Prisco D, Ruocco AL, Mastronardi P, Massa S, Di Minno G: Perioperative handling of patients on antiplatelet therapy with need for surgery. Intern Emerg Med. 2009;4: 279-88.

23 Dyszkiewicz-Korpanty A, Quinton R, Yassine J, Sarode R: The effect of a pneumatic tube transport system on PFA-100 trade mark closure time and whole blood platelet aggregation. $\mathrm{J}$ Thromb Haemost. 2004;2: 354-6. 


\title{
Chapter 4a
}

The effects of pneumatic tube system transport on ROTEM analysis and contact activation assessed by thrombin generation test

\author{
Marcus D. Lancé, Gerhardus J.A.J.M. Kuiper, Matthijs Sloep, \\ Henri M.H. Spronk, René van Oerle, Hugo ten Cate, Marco A.E. Marcus, Yvonne \\ M.C. Henskens
}

Based on: Thrombosis Research (2012),

Sep;130(3):e147-50 


\begin{abstract}
Background: Rotational thromboelastometry (ROTEM) is a popular point-of-care test for measuring normal or impaired coagulation and fibrin clot formation. It generates results quickly and may benefit individualised guided haemostatic therapy. However, processing of blood specimens by non-technicians might decrease the quality and reproducibility of ROTEM results. Centralised laboratory equipment receiving specimens through a pneumatic tube system (PTS) could avoid non-expert handling. This study aimed to evaluate the influence of PTS transport of blood samples on fibrin clot formation and thrombin generation triggered by tissue factor or contact activation.
\end{abstract}

Methods: Blood specimens from 44 patients were drawn immediately after arterial puncture. Samples were collected into citrate anticoagulant or into citrate/corn trypsin inhibitor (CTI), a factor XIla pathway inhibitor. Both types of samples were transported by either walking or PTS. Analysis of the recalcified citrated blood was performed with four different ROTEM tests. Plasmas from citrate and citrate/CTI blood were evaluated on thrombin generation with(out) tissue factor.

Results: Clot formation measurement using the different ROTEM tests revealed no more than minute differences between the way of transporting blood samples. Clot formation times were significantly shortened in samples sent by PTS for the EXTEM and NATEM tests of ROTEM, but the differences were negligible. Measurement of thrombin generation with or without tissue factor did not reveal any effect of tube transport via walking or PTS, neither in citrate nor in citrate/CTI plasma samples. Addition of $\mathrm{CTI}$ to the anticoagulant had a similar effect on thrombin generation in plasmas from blood transported via walking or PTS.

Conclusions: Whole-blood samples can be sent by PTS for ROTEM analysis. Transport via PTS does not result in a measurable increase in contact activation. Given that most hospitals have a different PTS, the present findings may not be valid for all hospitals. 


\section{Introduction}

Point-of-care laboratory monitoring is increasingly used in modern anaesthesiology. The main advantage of this monitoring is a rapid data acquisition, which enables to treat patients fast in a more individualised way. Because anaesthesia teams that are tending to bleeding patients are occupied with stabilising vital functions, the performing of additional laboratory tests might interfere with their regular workflow. There is also concern about the quality and reproducibility of laboratory tests done by non-technicians, especially when the results are critically important in decisionmaking [1]. An alternative to point-of-care monitoring in the operating theatre is to send blood specimens to the hospital laboratory with minimal time delay. Pneumatic tube systems (PTS) can reduce the clinical workload and shorten the turnaround time of hospital laboratories by quickly transporting blood samples. While most haematological indices and standard coagulation indices are not influenced by transport via PTS [2-4], there have been reports of haemolysis in serum samples and red blood cell concentrates due to the acceleration and deceleration of transport via PTS [5-7]. Haemolysis of red blood cells leads to the generation of microparticles, which have been shown to trigger thrombin generation in a factor XII-dependent manner [8]. Some authors interpret these changes as minor and without clinical consequences. However, there are good indications that the transport of specific specimens by PTS influences the measurement outcome, such as cerebral spinal fluid or blood samples to measure gases and platelet activation, using the PFA-100 system [9-13]. On the other hand, it was shown that PTS transport of citrated blood samples does not influcence platelet function, as assessed with multiple electrode aggregrometry [14].

The influence of blood transport via PTS on fibrin clot formation by rotational thromboelastometry has not been investigated. One of the drawbacks of PTS transport might be contact activation of blood samples in the test tubes [15]. Contact activation (via factor XIla) can be investigated by comparing thrombin generation in blood (plasma) samples collected into either citrate or citrate plus factor XIla pathway inhibitor, corn trypsin inhibitor (CTI). The aim of this study was to evaluate the influence of whole-blood transport via PTS on fibrin clot formation as assessed by ROTEM, and to determine if this transport leads to increased contact activation. 


\section{Methods}

After approval by the local ethics board, 44 patients scheduled for elective cardiothoracic surgery at the Maastricht University Medical Centre gave written informed consent and were included in the study. Exclusion criteria were use of anticoagulants other than acetylsalicylic acid, and age younger than 18 years. Upon arrival in the operating theatre, patients received standard monitoring for cardiac anaesthesia, including five lead electrocardiography, noninvasive blood pressure measurement and pulse oxymetry. Subsequently, a venous line (Vasofix Safety, 16G or 14G, Braun Melsungen, Germany) was inserted in the forearm, and an arterial line (Radial Artery Catheterization set, 20G, Arrow International, Reading, PA, USA) was positioned in the radial artery under subcutaneous local anaesthesia, using $1 \mathrm{~mL}$ of $1 \%$ lidocaine solution. Blood was drawn from the arterial line immediately after insertion. The first $10 \mathrm{~mL}$ were discarded before filling one tube, containing ethylenediamine tetraacetic acid anticoagulant (K-EDTA, BD Vacutainer Tubes, Plymouth, UK), two tubes containing citrate anticoagulant (Na-citrate 1.005 M, BD Vacutainer Tubes) and two tubes containing both Na-citrate and CTI $(40 \mu \mathrm{g} / \mathrm{mL}$, Haematologic Technologies, VE, USA). Two sets of labeled tubes, one citrate and one CTI tube, were taken to the central laboratory. One set was sent by PTS and the other set was transported by walking.

Platelet-poor plasma (PPP) was prepared by centrifugation of the blood at $2,000 \times g$ for 5 minutes, followed by a second centrifugation step at $10,000 \times \mathrm{g}$ for 10 minutes. Plasma aliquots were snapfrozen into liquid nitrogen, and stored at $-80{ }^{\circ} \mathrm{C}$ until analysis.

The PTS installed in the hospital (Swisslog-ErgoTrans, Apeldoorn, the Netherlands) connects the central surgical complex on the third floor to the haematological laboratory at the fifth floor. The circuit includes two switching stations for change of direction, and does not contain heat or cold sources. The system generates a maximum speed of $8 \mathrm{~m} / \mathrm{s}$. Transport time from the operating theatre to the laboratory is between 83 and 110 seconds.

Analysis of whole-blood samples for haematological parameters, i.e. haemoglobin, haematocrit and platelet count, was performed with a Coulter LH-750 analyser (Beckman Coulter, Woerden, the Netherlands). ROTEM analysis was performed using citrated blood samples by the following standard assays according to the manufacturer's recommendations at $37^{\circ} \mathrm{C}$ : NATEM (recalcification, no trigger), 
INTEM (recalcification with ellagic acid stimulation), EXTEM (recalcification and tissue factor stimulation) and FIBTEM (platelet distortion with cytochalasin $D$, recalcification and stimulation with tissue factor) [16].

To investigate whether transport via PTS causes contact activation via the factor XIla pathway, stored plasma samples were analyzed for thrombin generation. This was assessed using the calibrated automated thrombogram method (CAT, Thrombinoscope, Maastricht, the Netherlands) with as triggers $\mathrm{CaCl}_{2}$ and phospholipids $(4 \mu \mathrm{M})$ in the presence or absence of $1 \mathrm{pM}$ tissue factor. In all runs, normal pooled plasma was used as a reference, in order to reduce inter-assay variations of the time-independent curve parameters, i.e. endogenous thrombin potential (ETP) and peak height [17]. Considering a different of $20 \%$ in ROTEM analysis as clinically relevant, 44 samples were necessary to reach a statistical power of $90 \%$ with an $\alpha$-error of 0.05 .

Statistical analyses were performed using GraphPad Prism software (San Diego, CA, USA). After testing for normal distribution, a paired Student's t-test analysis was used for normally distributed variables, and the Wilcoxon signed-rank test was used for nonparametric variables were used to determine statistical significance. A p value $<0.05$ was considered to be significant.

\section{Results}

A total of 44 patients were included in this study. Thirty patients (68.2\%) were male and $14(31.8 \%)$ were female. Patient age was $69.7 \pm 11.5$ years (mean \pm SD). Haematological parameters revealed a haemoglobin level of $7.7 \pm 0.8 \mathrm{mM}$, a haematocrit of $0.38 \pm 0.04 \%$, and a platelet count of $210 \pm 60.1 \times 10^{9} / \mathrm{L}$.

All 44 pairs of citrated blood samples, after transport by PTS or walking, were analyzed on fibrin clot formation using four different ROTEM assays, INTEM, EXTEM, FIBTEM and NATEM. In the majority of thromboelastograph paraments no significant difference was detected between the blood samples carried by PTS or walking (Table 1). In some cases, though, there were minute differences that reached statistical significance. In the EXTEM assay with samples transported by PTS or walking, the clot formation time was $65 \pm 20$ seconds or $67 \pm 17$ seconds, and the alpha angle was $79 \pm 3^{\circ}$ or $77 \pm 3^{\circ}$, respectively. Similarly, in the NATEM assay, curves were slightly shortened in the samples sent by PTS. 
Table 1: Thromboelastography analysis of citrated whole blood samples from 44 patients, transported by either PTS or walking, using different ROTEM assays (INTEM, EXTEM, FIBTEM and NATEM).

\begin{tabular}{|c|c|c|c|c|c|c|}
\hline & \multicolumn{2}{|l|}{ INTEM } & \multirow[b]{2}{*}{$\begin{array}{l}P \text { - } \\
\text { value }\end{array}$} & \multicolumn{2}{|l|}{ EXTEM } & \multirow[b]{2}{*}{$\begin{array}{l}\mathrm{P}- \\
\text { value }\end{array}$} \\
\hline & PTS & Walking & & PTS & Walking & \\
\hline CT (s) & $135(97-172)$ & 137 (83-189) & 0.47 & 35 (24-49) & $35(18-57)$ & 0.93 \\
\hline $\mathrm{CFT}(\mathrm{s})$ & $64(36-103)$ & $63(40-99)$ & 0.54 & $65(36-119)$ & $67(33-107)$ & 0.02 \\
\hline Alpha angle $\left(^{\circ}\right)$ & $78(73-83)$ & $78(71-82)$ & 0.17 & $79(68-84)$ & $77(68-84)$ & 0.01 \\
\hline $\operatorname{MCF}(\mathrm{mm})$ & $62(51-76)$ & $62(52-75)$ & 0.56 & $63(51-77)$ & $62(53-77)$ & 0.08 \\
\hline \multirow[t]{3}{*}{ LI60 (\%) } & 91 (86-97) & $91(86-97)$ & 0.50 & 90 (79-98) & 91 (84-97) & 0.16 \\
\hline & FIBTEM & & & NATEM & & \\
\hline & PTS & Walking & $\begin{array}{l}\mathrm{P} \text { - } \\
\text { value }\end{array}$ & PTS & Walking & $\begin{array}{l}\text { P- } \\
\text { value }\end{array}$ \\
\hline CT (s) & $33(10-53)$ & $33(22-57)$ & 0.73 & $508(213-846)$ & $571(187-990)$ & 0.01 \\
\hline CFT(s) & $\mathrm{n} / \mathrm{a}$ & $\mathrm{n} / \mathrm{a}$ & $\mathrm{n} / \mathrm{a}$ & $127(46-263)$ & $136(54-305)$ & 0.03 \\
\hline Alpha angle $\left(^{\circ}\right)$ & $\mathrm{n} / \mathrm{a}$ & $\mathrm{n} / \mathrm{a}$ & $\mathrm{n} / \mathrm{a}$ & $67(47-80)$ & $65(44-79)$ & 0.04 \\
\hline $\operatorname{MCF}(\mathrm{mm})$ & $18(10-25)$ & $18(9-26)$ & 0.57 & $61(51-72)$ & $60(46-79)$ & 0.01 \\
\hline LI60 (\%) & $\mathrm{n} / \mathrm{a}$ & $\mathrm{n} / \mathrm{a}$ & $\mathrm{n} / \mathrm{a}$ & $93(85-98)$ & $92(86-97)$ & 0.82 \\
\hline
\end{tabular}

Abbreviations of curve parameters: CT, clotting time; CFT, clot formation time, MCF, maximum clot firmness, LI60, lysis index at 60 minutes. N/a, not applicable. Data are presented as means (interquartile ranges).

Thrombin generation analysis was performed on plasmas derived from blood samples of 31 of the 44 patients, collected into citrate or into citrate/CTI. Again, the effect of blood transport by walking or PTS was studied. Blood from 13 patients was not used, because citrate/CTI tubes were not available. Regardless of the transport method, there was a similar inhibition of thrombin generation curves in all samples drawn into citrate/CTI, when compared to citrate tubes (Table 2). This difference was more pronounced, when thrombin generation was triggered with $\mathrm{CaCl}_{2}$ in the absence of tissue factor. Notably, in this case, blood collection into citrate/CTI completely abolished the thrombin generation, thus pointing to appreciable contact activation in the blood samples collected in citrate alone. Also upon triggering with $\mathrm{CaCl}_{2}$ and $1 \mathrm{pM}$ tissue factor, thrombin generation was still suppressed in the samples derived from CTI tubes, independently of the method of blood transport. Again the citrate tubes showed similar signs of contact activation in both groups (transport by walking or PTS). 
Table 2: Calibrated automated thrombogram (CAT) analysis of plasmas from blood samples drawn into citrate of citrate/CTI and transported to the laboratory by PTS of walking.

\begin{tabular}{|c|c|c|c|c|}
\hline & \multicolumn{2}{|c|}{ Citrate - TF } & \multicolumn{2}{|c|}{ Citrate/CTI - TF } \\
\hline & PTS & Walking & PTS & Walking \\
\hline $\begin{array}{l}\text { Lag time (min) } \\
\text { Peak height }\end{array}$ & $12(10-15)$ & $13(10-17)$ & 99 (38-99) & $62(28-99)$ \\
\hline $\begin{array}{l}\text { (nM) } \\
\text { ETP (nM.min) }\end{array}$ & $\begin{array}{c}292(179-331) \\
1278(791-1450)\end{array}$ & $\begin{array}{c}296(218-333) \\
\end{array}$ & $\begin{array}{l}1(0-1) \\
0(0-0)\end{array}$ & $\begin{array}{l}1(0-1) \\
0(0-0)\end{array}$ \\
\hline VI (nM/min) & $\begin{array}{c}12 / 8(191-1450) \\
137(78-172)\end{array}$ & $\begin{array}{c}1287(957-1386) \\
144(91-167)\end{array}$ & $0(0-0)$ & $\begin{array}{l}0(0-0) \\
0(0-0)\end{array}$ \\
\hline & \multicolumn{2}{|c|}{ Citrate + TF } & \multicolumn{2}{|c|}{ Citrate/CTI + TF } \\
\hline & PTS & Walking & PTS & Walking \\
\hline $\begin{array}{l}\text { Lag time }(\min ) \\
\text { Peak height }(\% \text { o } \\
\text { normal) } \\
\text { ETP }(\% \text { of normal }\end{array}$ & $\begin{array}{c}3(3-4) \\
183(116-206) \\
120(89-134)\end{array}$ & $\begin{array}{c}3(3-4) \\
183(134-208) \\
118(95-134)\end{array}$ & $\begin{array}{l}74(53-103) \\
82(72-101)\end{array}$ & $\begin{array}{c}4(4-5) \\
74(55-111) \\
84(74-101)\end{array}$ \\
\hline VI (nM/min) & $86(60-120)$ & $88(56-121)$ & $21(14-34)$ & $20(14-36)$ \\
\hline
\end{tabular}

Platelet-poor plasma derived whole blood collected in citrate or citrate/CTI was triggered with $\mathrm{CaCl}_{2}$ in the presence or absence of tissue factor (TF, $1 \mathrm{pM}$ ). Where indicated, thrombin generation parameters are expressed as percentage values compared to normal pooled plasma. Data are presented as median values (interquartile ranges), $n=31$. ETP, endogenous thrombin potential (area-under-thecurve), $\mathrm{VI}=$ velocity index (tangent between end lag time and peak height), Wilcoxon signed-rank tests did not show significant differences beween the two groups.

\section{Discussion}

The present study demonstrates that PTS transport of platelet concentrates does not inflict clinically relevant consequences on ROTEM analysis results. Even though the collection of blood samples in citrate leads to a measurable degree of contact activation, as measured by comparison with citrate/CTI samples in the CAT assay, this did not appear to be increased by PTS transport, as compared to walking transport to the laboratory. Management of acute haemorrhage demands swift reaction and clinical decision-making, and assaying of whole-blood clot formation by ROTEM analysis has been shown to successfully assist in this. Several authors recommend transfusing haemorrhagic patients as quickly as possible, even without knowledge of haemostatic properties, in order to prevent haemodilution and aggravation of bleeding [18]. Others state that ROTEM analysis provides important information on the coagulant state in acute bleeding patients [19-21]. It is hence debated whether to perform ROTEM analysis directly at the bedside (i.e. the operating theatre or emergency department) or more distantly in the central hospital 
laboratory. The main argument supporting bedside testing is fast availability of results for tailored haemotherapy. However, concerns arise on the quality of such point-ofcare results, and some authors recommend performing laboratory analysis by trained technicians in the hospitals central laboratory [22-25].

The main argument against ROTEM assaying in a central laboratory is the time needed to transport blood samples. Transport via PTS is quicker, and reduces this time. However, transport in pneumatic systems may lead to platelet activation and contact activation [6, 9, 13]. For this reason, PTS transport has not been recommended for several point of care tests [11,13]. Wallin and colleagues describe significant shortening of clot formation using the thromboelastograph (TEG), when they compared PTS with walking transport [26]. Unfortunately, the authors do not describe how coagulation was triggered in the assay. This finding is reflected by a minor shortening of clot forming time in the present EXTEM and NATEM tests after transport via PTS. Another recent study revealed no significant differences in ROTEM analysis, when PTS transport was performed [24]. However, it should be noted that marked differences exist between the PTS used in different hospitals, in terms of switching points, speed and acceleration/ deceleration. Another possible reason for the minute differences between PTS and walking transport might be activation of platelets. However, platelet activity is poorly reflected in ROTEM analysis [27].

The thrombin generation assay can detect small changes in the capacity of the coagulation system $[28,29]$. This assay was used to investigate the possibility of contact activation induced by PTS. Addition of CTI, a specific factor XIla inhibitor, to citrate tubes during blood drawing eliminates contact activation. However, no clear influence of transport via PTS could be detected.

An acutely bleeding patient requires intense manpower support on the scene, limiting the time available to perform point-of-care tests by team members themselves. In addition, working under pressure might affect the laboratory results [30], and generate variability in the test outcome [25, 31]. Kitchen et al. demonstrated a variability in ROTEM results between 7 and $84 \%$ in point-of-care settings in various hospitals [25]. Because of these reasons, it is preferable to maintain carrying out whole-blood tests like ROTEM in the hospital laboratory. In conclusion, our study demonstrated that analysis of blood samples via ROTEM is hardly influenced by transport via PTS. In addition, PTS transport did not result in notable contact 
activation via the factor XIla pathway. However, as the PTS can markedly differ between hospitals, the present findings may not be applicable to all hospitals.

\section{References}

1 Chitlur M, Lusher J: Standardization of thromboelastography: values and challenges. Semin Thromb Hemost. 2010;36: 707-11.

2 Guss DA, Chan TC, Killeen JP: The impact of a pneumatic tube and computerized physician order management on laboratory turnaround time. Ann Emerg Med. 2008;51: 181-5.

3 Fernandes CM, Worster A, Eva K, Hill S, McCallum C: Pneumatic tube delivery system for blood samples reduces turnaround times without affecting sample quality. J Emerg Nurs. 2006;32: 139-43.

4 Keshgegian AA, Bull GE: Evaluation of a soft-handling computerized pneumatic tube specimen delivery system. Effects on analytical results and turnaround time. Am J Clin Pathol. 1992;97: 535-40.

5 Ando H, Nieminen K, Toppila E, Starck J, Ishitake T: Effect of impulse vibration on red blood cells in vitro. Scand J Work Environ Health. 2005;31: 286-90.

6 Sodi R, Darn SM, Stott A: Pneumatic tube system induced haemolysis: assessing sample type susceptibility to haemolysis. Ann Clin Biochem. 2004;41: 237-40.

7 Stair TO, Howell JM, Fitzgerald DJ, Bailey SC, Bastasch MD: Hemolysis of blood specimens transported from ED to laboratory by pneumatic tube. Am J Emerg Med. 1995;13: 484.

8 van der Meijden PE, van Schilfgaarde M, van Oerle R, Renne T, Ten Cate H, Spronk HM: Platelet- and erythrocyte-derived microparticles trigger thrombin generation via Factor XIla. J Thromb Haemost. 2012. in press.

9 Collinson PO, John CM, Gaze DC, Ferrigan LF, Cramp DG: Changes in blood gas samples produced by a pneumatic tube system. J Clin Pathol. 2002;55: 105-7.

Wenham PR, Hanson T, Ashby JP: Interference in spectrophotometric analysis of cerebrospinal fluid by haemolysis induced by transport through a pneumatic tube system. Ann Clin Biochem. 2001;38: 371-5.

11 Dyszkiewicz-Korpanty A, Quinton R, Yassine J, Sarode R: The effect of a pneumatic tube transport system on PFA-100 trade mark closure time and whole blood platelet aggregation. J Thromb Haemost. 2004;2: 354-6.

12 Hubner U, Bockel-Frohnhofer N, Hummel B, Geisel J: The effect of a pneumatic tube transport system on platelet aggregation using optical aggregometry and the PFA-100. Clin Lab. 2010;56: 59-64.

13 Bolliger D, Seeberger MD, Tanaka KA, Dell-Kuster S, Gregor M, Zenklusen U, Grapow M, Tsakiris DA, Filipovic M: Pre-analytical effects of pneumatic tube transport on impedance platelet aggregometry. Platelets. 2009;20: 458-65.

14 Braun S, von Beckerath N, Ellert J, Kastrati A, Schomig A, Vogt W, Sibbing D: Assessment of platelet function in whole blood by multiple electrode aggregometry: transport of samples using a pneumatic tube system. Am J Clin Pathol. 2009;132: 802-3; author reply 3-4.

15 Spronk HM, Dielis AW, Panova-Noeva M, van Oerle R, Govers-Riemslag JW, Hamulyak K, Falanga A, Cate HT: Monitoring thrombin generation: is addition of corn trypsin inhibitor needed? Thromb Haemost. 2009;101: 1156-62.

16 Mittermayr M, Streif W, Haas T, Fries D, Velik-Salchner C, Klingler A, Oswald E, Bach C, Schnapka-Koepf M, Innerhofer P: Hemostatic changes after crystalloid or colloid fluid administration during major orthopedic surgery: the role of fibrinogen administration. Anesth Analg. 2007;105: 905-17, table of contents.

17 Spronk HM, Dielis AW, De Smedt E, van Oerle R, Fens D, Prins MH, Hamulyak K, ten Cate H: Assessment of thrombin generation II: Validation of the Calibrated Automated Thrombogram in platelet-poor plasma in a clinical laboratory. Thromb Haemost. 2008;100: 362-4.

18 Johansson PI, Stensballe J: Effect of Haemostatic Control Resuscitation on mortality in massively bleeding patients: a before and after study. Vox Sang. 2009;96: 111-8.

19 Frith D, Brohi K: The acute coagulopathy of trauma shock: clinical relevance. Surgeon. 2010;8: 159-63.

20 Johansson PI, Stensballe J: Hemostatic resuscitation for massive bleeding: the paradigm of plasma and platelets--a review of the current literature. Transfusion. 2010;50: 701-10. 
21 Rugeri L, Levrat A, David JS, Delecroix E, Floccard B, Gros A, Allaouchiche B, Negrier C: Diagnosis of early coagulation abnormalities in trauma patients by rotation thrombelastography. J Thromb Haemost. 2007;5: 289-95.

22 du Plessis M, Ubbink JB, Vermaak WJ: Analytical quality of near-patient blood cholesterol and glucose determinations. Clin Chem. 2000;46: 1085-90.

23 Briedigkeit L, Muller-Plathe $\mathrm{O}$, Schlebusch $\mathrm{H}$, Ziems J: Recommendations of the German Working Group on medical laboratory testing (AML) on the introduction and quality assurance of procedures for Point-of-Care Testing (POCT) in hospitals. Clin Chem Lab Med. 1999;37: 919-25.

24 Colucci G, Giabbani E, Barizzi G, Urwyler N, Alberio L: Laboratory-based ROTEM((R)) analysis: implementing pneumatic tube transport and real-time graphic transmission. Int $\mathrm{J}$ Lab Hematol. 2011;33: 441-6.

25 Kitchen DP, Kitchen S, Jennings I, Woods T, Walker I: Quality assurance and quality control of thrombelastography and rotational Thromboelastometry: the UK NEQAS for blood coagulation experience. Semin Thromb Hemost. 2010;36: 757-63.

26 Wallin O, Soderberg J, Grankvist K, Jonsson PA, Hultdin J: Preanalytical effects of pneumatic tube transport on routine haematology, coagulation parameters, platelet function and global coagulation. Clin Chem Lab Med. 2008;46: 1443-9.

27 Gorlinger K, Jambor C, Dirkmann D, Dusse F, Hanke A, Adamzik M, Hartmann M, Philipp S, Weber AA, Rahe-Meyer N: [Platelet function analysis with point-of-care methods]. Herz. 2008;33: 297-305.

28 Hemker HC, Al Dieri R, De Smedt E, Beguin S: Thrombin generation, a function test of the haemostatic-thrombotic system. Thromb Haemost. 2006;96: 553-61.

29 Ten Cate H: Thrombin generation in clinical conditions. Thrombosis research. 2011.

30 Croskerry P, Abbass A, Wu AW: Emotional influences in patient safety. J Patient Saf. 2010;6: 199-205.

31 Mauch J, Spielmann N, Hartnack S, Madjdpour C, Kutter AP, Bettschart-Wolfensberger R, Weiss $\mathrm{M}$, Haas $\mathrm{T}$ : Intrarater and interrater variability of point of care coagulation testing using the ROTEM delta. Blood Coagul Fibrinolysis. 2011. 


\section{Chapter 4b}

Platelet concentrate transport in pneumatic tube systems - does it work?

Marcus D. Lancé, Marco A. E. Marcus, René van Oerle, Maurice H. S. Theunissen, Yvonne M. C. Henskens

Based on:

Vox Sang. 2012 Jul; 103 (1): 79-82 
Abstract: The use of pneumatic tube systems (PTS) for transport of blood samples in hospitals is generally accepted and reduces both workload and turnaround time of test results. PTS can also be used for the transport of blood products for transfusion purposes. How transport via PTS affects platelet concentrates is not known. In this paper, platelet functionality was investigated after single and multiple transports via PTS of platelet concentrates (non-radiated or irradiated), in comparison to storage of the concentrates. Before and after transport, platelet count, $\mathrm{pH}$, optical aggregation (LTA), impedance aggregation (MEA), P-selectin (CD-62P), and microparticles were measured. It was found that platelet metabolic markers changed as a result of sample storage but not by using PTS. Platelet function markers were not different in concentrates sent by PTS with the exception of the thrombin receptor-activating peptide (TRAP)-induced platelet aggregation measured by LTA or MEA after multiple PTS transport. This work shows that single PTS transport of PC concentrates from the laboratory to the operation theatre is possible without detectable loss in platelet function. 


\section{Introduction}

Pneumatic tube systems (PTS) reduce the workload and turnaround time of clinical laboratories by speeding up the transport of blood samples and making it less dependent on manpower. While most haematology and coagulation parameters are not known to be influenced by transport via PTS [1-3], there are reports of haemolysis in red blood cell concentrates, due to the acceleration and deceleration of transport by PTS and due to vibration of the samples [4-6]. Most authors interpret these changes as being minor and without clinical effect. Yet, the transport by PTS of specimens for blood gas analysis, cerebral spinal fluid analysis, or platelet function analysis by PFA-100 is not recommended, because results might be unreliable [711]. All transfusion products, and especially platelet concentrates, should be of the highest quality possible, because recipients often have a tendency to bleed, which conveys a high morbidity and mortality [12]. While the quality of platelet concentrates (PCs) is affected by storage and irradiation [13-16], it is not known whether it is affected by transport via PTS. To date, such transport has not been recommended because of an expected risk of platelet activation and a diminished functionality of the PCs after transfusion. The aim of this study was to evaluate the effects of PTS transport on the in vitro platelet function in PCs (irradiated and non-irradiated) and compare these effects to storage influences (maximum of 7 days).

\section{Materials and methods}

\section{Study design}

After approval of the local ethics committee (Maastricht University Medical Centre+, Maastricht, the Netherlands) two prospective single centre studies were performed. In PCs prepared for transfusion the in vitro platelet function was investigated.

In study 1 the effect of PTS and storage was investigated on day 2 and 7 after platelet collection, 12 PCs were transported via the PTS. Six out of 12 PCs were irradiated (Gammacell 2000-Canberra Eurisys, 25Gy) prior to transport and six were not treated (Figure 1).

In study $\mathbf{2}$ the effect of multiple transports was investigated. For this purpose, another two non-irradiated PCs were each divided equally into four samples (A, B, C, $D$ ), and sent through the PTS several times on different days in order to investigate 
the influence of transport frequency. Unit A was never sent and served as control. Unit $B$ was sent once on day 2, unit $C$ was sent once on days 2, 3, 6 and 7, and unit D was sent three times on days 2, 3, 6 and 7 (Figure 1). Samples were taken from each unit after transport. All activities were performed in accordance with institutional guidelines.

\section{Platelet concentrates}

The local blood bank (Sanquin Blood Services the Netherlands) provided 14 fresh (age: maximal 24 hours after donation) PCs. Each PC consisted of randomly pooled platelet buffy coats in plasma $\left(200-400 \mathrm{~mL}\right.$ volume, $250-350 \times 10^{9}$ platelet $/ \mathrm{L},<1$ $\times 10^{6}$ leucocytes per unit) derived from five healthy volunteers by the buffy coat method and processed according to the guidelines of our blood bank and European recommendations. For study $\mathbf{1}, 12$ original PCs were used. For study 2 , at the start of the experiment, two non-irradiated PCs were carefully resuspended and divided at equal volumes into four smaller sample bags (pediatric platelet storage bags) and closed under sterile conditions. The concentrates did not pass the maximal storage life of PCs in Europe of 7 days. During the experiments, all PCs were agitated gently and continuously in a single layer, temperature controlled automatic platelet agitator at $22^{\circ} \mathrm{C}[17]$.

\section{Pneumatic Tube Transport system}

The PTS used (Swisslog-ErgoTrans, Apeldoorn, the Netherlands) connects the laboratory on the fifth floor with the central surgical complex on the third floor and is kept at room temperature. The PTS consists of two switching stations for change of direction. The system generates a maximum speed of $8 \mathrm{~m} / \mathrm{s}$. Transport time from the laboratory to the operating theatres is between 83 and 110 seconds. At arrival, the transport box falls about $40-50 \mathrm{~cm}$ into a cushioned catch tray. 


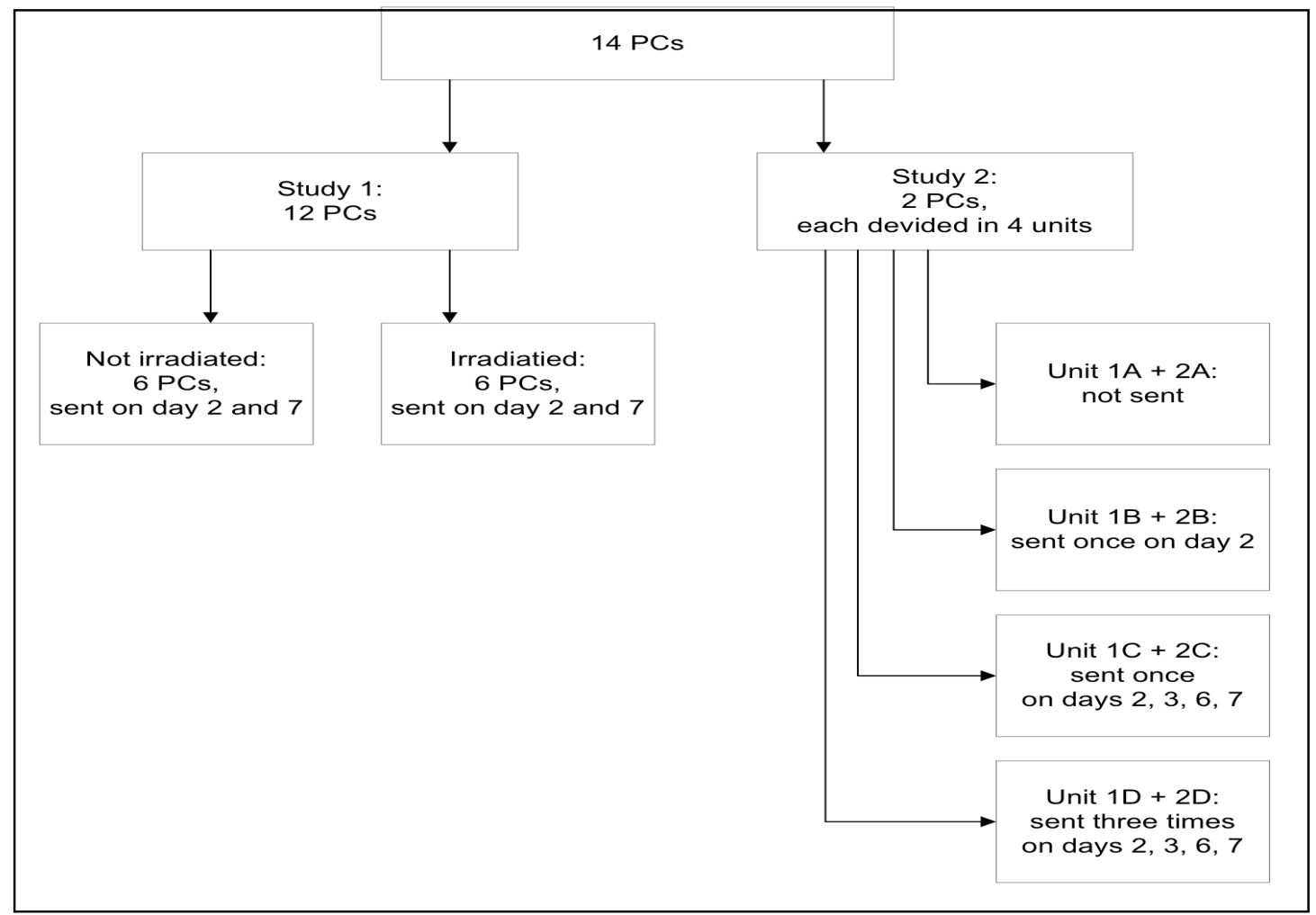

Figure 1: Scheme of dividing and sending of the platelet concentrates (PC) in study 1 and study 2.

\section{Sample analysis}

For both studies samples were taken from each bag before and after transport, using standard citrated vacuum tubes (sodium citrate $1.005 \mathrm{M}$, Vacutainer, Plymouth, UK). In these samples, platelet count and mean platelet volume (MPV) were measured using a Beckman Coulter (LH-750, Beckman Coulter, Woerden, the Netherlands). Glucose and lactate concentrations and $\mathrm{pH}$ were measured with a Chiron rapid lab 850 blood gas analyser (Siemens Healthcare Diagnostics, Breda, the Netherlands). Platelet function was measured by using two aggregation methods: light transmission aggregation (LTA) using an aggregometer and agonists from Kordia Life Science (Leiden, The Netherlands) and by multiple electrode aggregometry (MEA) using the Dynabyte Multiplate (Munich, Germany) and different agonists: collagen $[4 \mu \mathrm{g} / \mathrm{mL}$ (LTA) and 3.2 (MEA) $\mu \mathrm{g} / \mathrm{mL}$ ], thrombin receptor activating peptide [TRAP, $30 \mu \mathrm{M}$ (LTA) and $32 \mu \mathrm{M}$ (MEA)], adenosine diphosphate [ADP, $10 \mu \mathrm{M}$ (LTA) and $6.5 \mu \mathrm{M}$ (MEA)], and arachidonic acid [1 $\mathrm{mM}$ sodium arachidonic acid, NAA (LTA) and arachidonic acid-ASPI (MEA].

P-selectin (CD62) was measured with a flow cytometer (Coulter Epics XL, Beckman Coulter). The proportion of platelets positive for P-selectin was determined 
and compared with that of a control sample from healthy donors. Microparticles (MP) were measured by ELISA (ZYMUPHEN, MP-activity kit, Aniara, Mason, OH, USA) according to the manufacturer's instructions.

\section{Statistical analysis}

Statistical analyses were performed using SPSS Version 17 (SPSS, Chicago, IL, USA) and GraphPad Prism (GraphPad Software, San Diego, CA, USA). Wilcoxon signed ranks analysis and two-way ANOVA and multi-regression analysis was used. A p-value of $<0.05$ was considered to be statistically significant.

\section{Results}

\section{Study 1: Effects of single PTS transport}

General platelet function markers such as the mean platelet count, MPV, glucose, $\mathrm{pH}$ and lactate concentrations of the 12 PCs (irradiated or non-irradiated) were not influenced by PTS transport on day 2 or day 7 (Table 1). PTS transport of all PCs (days 2 and 7) had no significant influence on in vitro platelet function, as measured with collagen-, TRAP-, NAA- or ADP-induced aggregation using LTA (Figure 2A). Similar results were obtained using impedance aggregometry, MEA (Figure 2B). Fractions of platelets expressing CD62P and levels of microparticles in plasma were not changed after PTS. In contrast, platelet storage (comparison between day 2 and 7) did significantly affect the platelet function markers (except for $\mathrm{pH}$ ) in both irradiated and non-irradiated PCs, increased CD62P expression and reduced platelet aggregation, especially with the agonists ADP and collagen (Table 1 and Figure 2). 
Table 1: Results of study 1: comparison of functional markers of platelet concentrates (PCs) before and after PTS transport, and after different times of storage.

\begin{tabular}{|l|l|l|l|l|}
\hline & \multicolumn{2}{|l|}{ PTS transport } & \multicolumn{2}{l|}{ Storage time } \\
\hline Parameter & before & after & D2 & D7 \\
\hline Platelet count (10 $/ \mathrm{L})$ & $1130(142)$ & $1123(140)$ & $1130(142)$ & $1037(134)^{*}$ \\
\hline MPV (fL) & $7.1(0.4)$ & $7.1(0.4)$ & $7.13(0.38)$ & $7.46(0.33)^{*}$ \\
\hline $\mathrm{pH}$ & $7.2(0.0)$ & $7.2(0.0$ & $7.1(0.0)$ & $7.2(0.1)$ \\
\hline Glucose (mmol/L) & $21.3(1.5)$ & $21.3(1.2)$ & $20.6(15.9)$ & $15.88(1.6)^{*}$ \\
\hline Lactate (mmol/L) & $7.9(1.1)$ & $8.1(1.3)$ & $7.45(1.2)$ & $15.12(3.0)^{*}$ \\
\hline CD62P (\% positive) & $1.7(0.8)$ & $1.5(0.7)$ & $1.69(0.8)$ & $3.6(1.2)^{*}$ \\
\hline Microparticles & $22.5(16.6)$ & $21.9(15.7)$ & $22.5(16.6)$ & $45.5(50.1)^{*}$ \\
\hline
\end{tabular}

Storage time for 2 (D2) or 7 (D7) days. MPV (mean platelet volume). Data are means (S.D.), n=12. ${ }^{*} \mathrm{P}<0.05$.

\section{Study 2: Effects of multiple PTS transports}

Platelet function analysis with LTA showed that multiple PTS transport only influenced TRAP-induced platelet aggregation (frequency $p=0.03$, aging $p<0.0001$ ), whereas collagen-, arachidonic acid-, and ADP-induced platelet aggregation was not changed by multiple PTS transports (Figure $3 A$ ). Analysis of platelet function with MEA showed similar results for TRAP (Figure 3B). Similarly, CD62P expression and microparticle levels were not influenced by multiple PTS transports $(p=0.35$ and $p=0.58$, respectively, Table 1). In contrast, storage for 2-7 days influenced all platelet function markers significantly. 

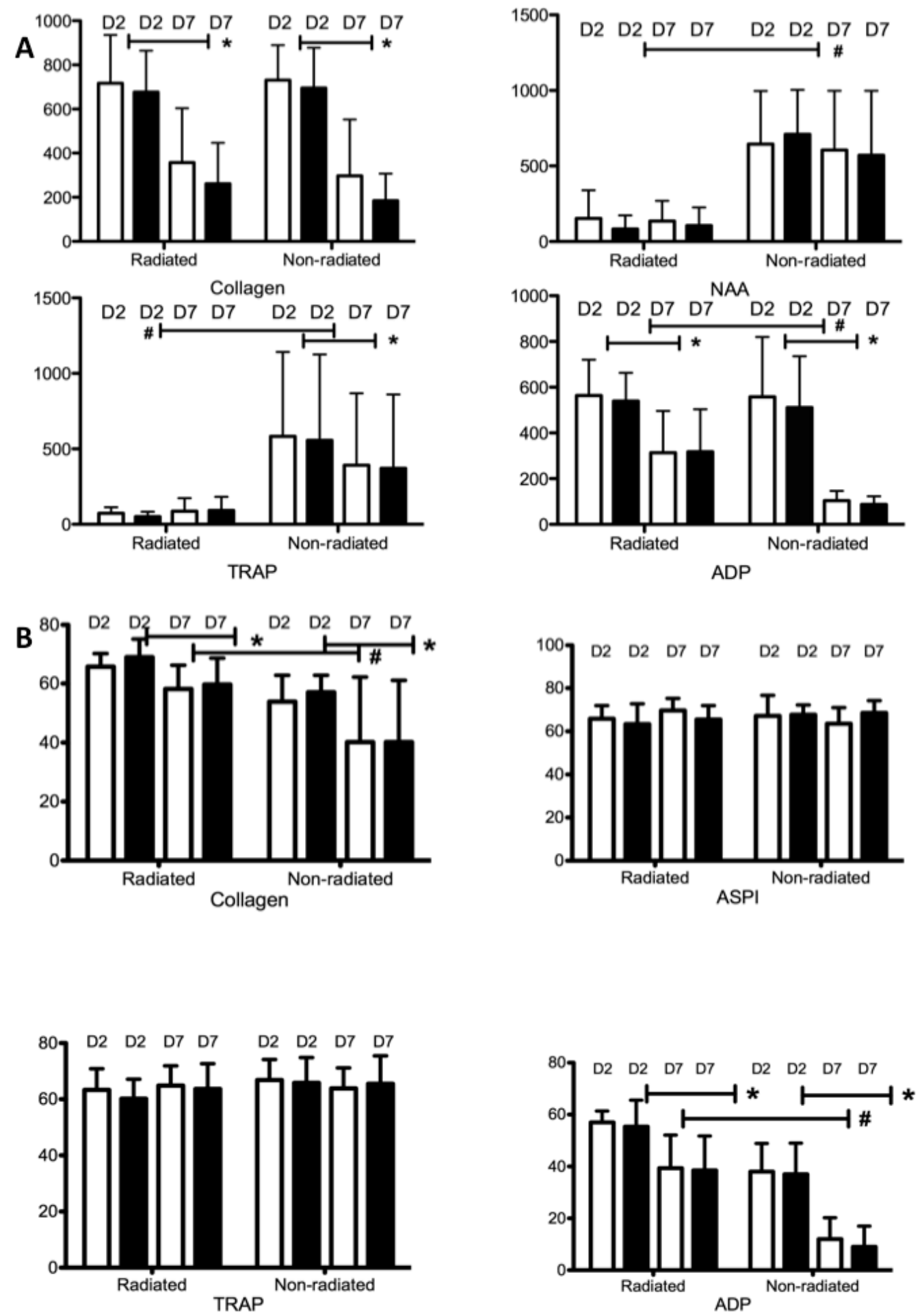

Figure 2: Study 1 aggregometry results. Bars represent means \pm S.D. White bars: before PTS; Black bars: after PTS; D2, storage day 2; D7, storage day 7. Panel A: LTA of PCs stimulated with collagen, arachidonic acid (NAA), thrombin receptor activating peptide (TRAP) or adenosine diphosphate (ADP). Panel B: MEA of PCs stimulated with collagen, arachidonic acid (ASPI), TRAP) or ADP. *Significant effect of storage time $(P<0.05),{ }^{\#}$ significant effect of radiation $(P<0.05)$. 
Collagen

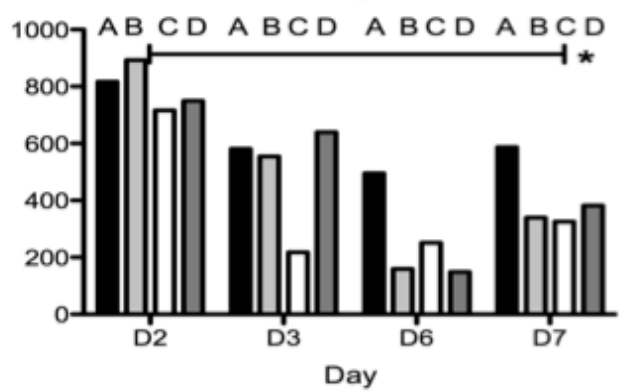

TRAP

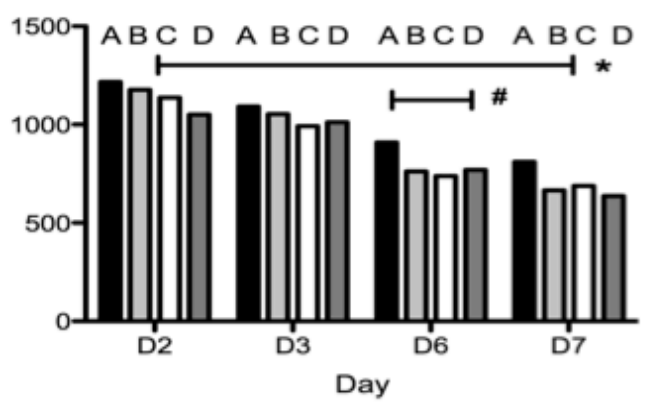

ADP

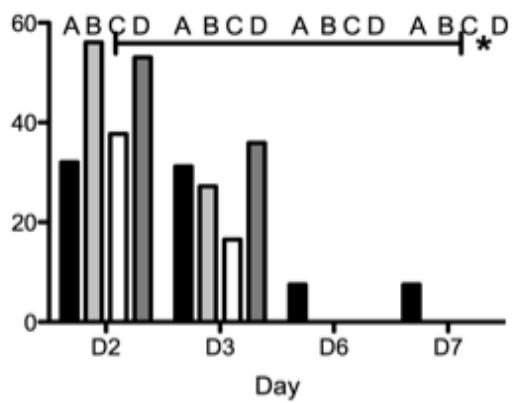

TRAP

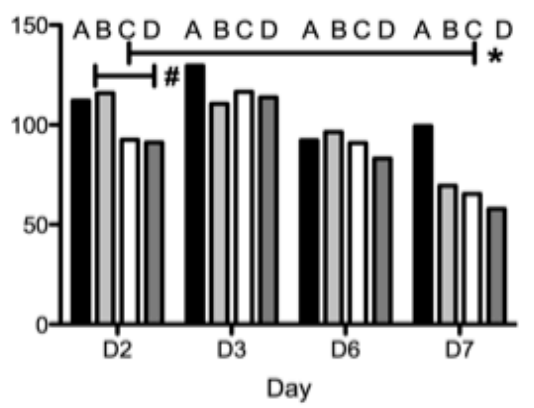

NAA

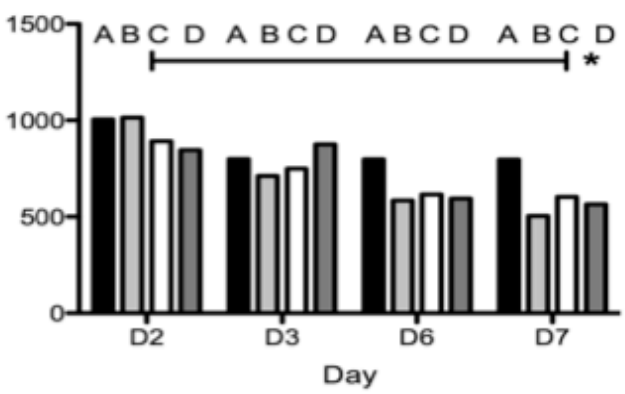

ADP

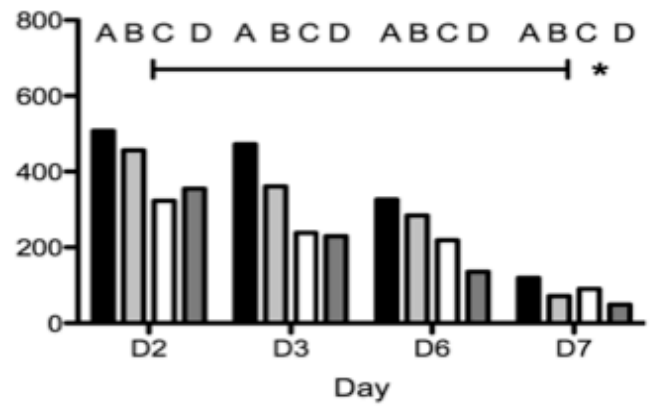

ASPI

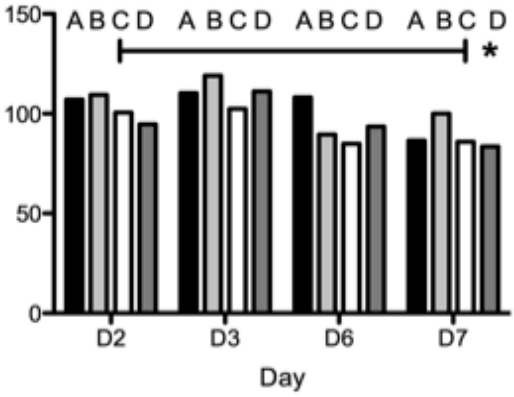

Collagen

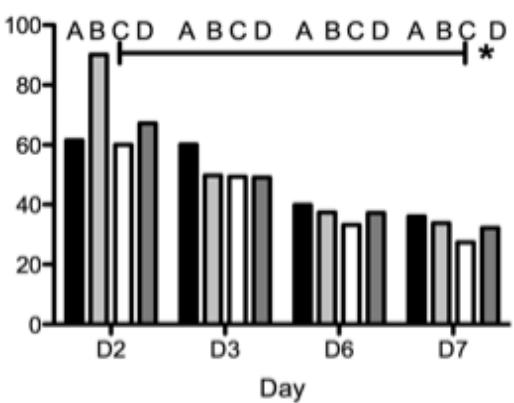

Figure 3: Study 2 aggregometry results. Bars represent means \pm S.D.; D: day. A, black, non sent; B, light grey, once sent; $C$, white, once daily sent; $D$, dark grey, thrice sent. Panel A : LTA of PCs stimulated with collagen, arachidonic acid (NAA), thrombin receptor activating peptide (TRAP) or adenosine diphosphate (ADP). Panel B: MEA of PCs stimulated with collagen, arachidonic acid (ASPI), TRAP or ADP. *Significant effect of storage time $(P<0.05),{ }^{*}$ significant effect of frequency of PTS use $(P<0.05)$. 


\section{Discussion}

This work shows that PTS transport of PC concentrates from the laboratory to the operation theatre is possible without detectable loss in platelet function. Platelets are susceptible to damage caused by excessive shaking, sudden accelerationdeceleration, and even vibration $[4,18]$. For this reason, several investigators do not recommend sending specimens by PTS for platelet function testing. High quality of blood products for transfusion [17], is very important and this includes optimal storage and transport conditions [15]. For example, Keuren and Albanyan showed that the type of storage medium influences platelet function [14, 19], while Akay concluded that storage bags influenced platelet function [20]. Comparable publications on the influence of transport of platelet concentrates before transfusions are scarce. Javela and co-workers reported that inter-hospital car transport did not influence platelet function of PCs under storage, as assessed by flow cytometry (expression of CD62P, CD63 and glycoprotein V) or from metabolic markers (glucose and lactate concentrations, $\mathrm{pH}$ ), but they did not assess platelet function by aggregometry. On the other hand, these authors found that storage did affect these markers, from which they concluded that platelet function should be monitored after inter-hospital car transport of PCs, to ensure the quality of the transfusion product [21].

We did not find evidence that single or multiple PTS transports influence platelet function, as measured by aggregometry (LTA or MEA), with the exception of a slightly decreased TRAP-induced aggregation after multiple transports of the PC samples. We can only compare our data to those of publications on whole blood sample transport by PTS. Thus, Braun et al. sent hirudin-anticoagulated whole blood samples via PTS and demonstrated that this transport did not influence platelet aggregation by MEA [22]. Wallin and colleagues also found that PTS transport of citrated whole blood samples did not influence platelet activity, measured with the PFA-100 system [23]. In contrast, Bolliger and coworkers found significant decreases in platelet function (measured by MEA) of hirudin-anticoagulated whole blood specimens after PTS transport, when compared with samples that had not been transported [11]. Similar effects are reported by Dyzkiewiscz-Korpanty and Hubner, who measured platelet activity by PFA-100 and LTA in citrated whole blood samples $[9,10]$. It should be noted that the whole blood samples of these studies contained 
red cells that are able to activate platelets by direct by ADP release, whereas we used PCs from which red reds had been removed. The contradictory results in the literature may be due to differences in pneumatic tube systems with different lengths, transportation speeds, connection points, transport temperature and storage times used, leading to a different exposure of PCs to mechanical stress and temperature drift.

We found a small effect that platelet aggregation with TRAP, but not with other agonists, was decreased after multiple PTS transports of PCs. This may be coincidence or indicate a slight sensitivity of TRAP-induced platelet activation to platelet PTS transport [24, 25]. Expression of P-selectin (CD62P) is considered to be a reliable marker of platelet activation [14, 19, 26, 27]. This parameter was markedly altered by PC storage, as reported earlier [14, 19, 28]. Microparticles are released upon platelet activation and facilitate coagulation [29]. PC transport did not influence the plasma level of microparticles, whereas storage significantly increased this level. Rank et al. also showed microparticles derived from activated platelets increased during storage [28], while Apelseth and co-workers reported higher levels of CD62P and microparticles during PC storage, as well as higher levels of CD62P and impaired TRAP- and collagen-induced platelet aggregation after irradiation [13]. We found that platelet counts and metabolic markers (glucose and lactate concentrations, $\mathrm{pH}$ ) were stable after PTS transport, remaining within the ranges required by blood banks $[16,17]$.

In conclusion, our results show that PTS transport of PCs is feasible and has a negligible effect on in vitro platelet function. It remains to be demonstrated whether PTS transport of PCs influences platelet function in patients transfused with this product. 


\section{References}

1 Guss DA, Chan TC, Killeen JP: The impact of a pneumatic tube and computerized physician order management on laboratory turnaround time. Ann Emerg Med. 2008;51: 181-5.

2 Fernandes CM, Worster A, Eva K, Hill S, McCallum C: Pneumatic tube delivery system for blood samples reduces turnaround times without affecting sample quality. J Emerg Nurs. 2006;32: 139-43.

3 Keshgegian AA, Bull GE: Evaluation of a soft-handling computerized pneumatic tube specimen delivery system. Effects on analytical results and turnaround time. Am J Clin Pathol. 1992;97: 535-40.

4 Ando H, Nieminen K, Toppila E, Starck J, Ishitake T: Effect of impulse vibration on red blood cells in vitro. Scand J Work Environ Health. 2005;31: 286-90.

5 Sodi R, Darn SM, Stott A: Pneumatic tube system induced haemolysis: assessing sample type susceptibility to haemolysis. Ann Clin Biochem. 2004;41: 237-40.

6 Stair TO, Howell JM, Fitzgerald DJ, Bailey SC, Bastasch MD: Hemolysis of blood specimens transported from ED to laboratory by pneumatic tube. Am J Emerg Med. 1995;13: 484.

7 Collinson PO, John CM, Gaze DC, Ferrigan LF, Cramp DG: Changes in blood gas samples produced by a pneumatic tube system. J Clin Pathol. 2002;55: 105-7.

8 Wenham PR, Hanson T, Ashby JP: Interference in spectrophotometric analysis of cerebrospinal fluid by haemolysis induced by transport through a pneumatic tube system. Ann Clin Biochem. 2001;38: 371-5.

9 Dyszkiewicz-Korpanty A, Quinton R, Yassine J, Sarode R: The effect of a pneumatic tube transport system on PFA-100 trade mark closure time and whole blood platelet aggregation. J Thromb Haemost. 2004;2: 354-6.

10 Hubner U, Bockel-Frohnhofer N, Hummel B, Geisel J: The effect of a pneumatic tube transport system on platelet aggregation using optical aggregometry and the PFA-100. Clin Lab. 2010;56: 59-64.

11 Bolliger D, Seeberger MD, Tanaka KA, Dell-Kuster S, Gregor M, Zenklusen U, Grapow M, Tsakiris DA, Filipovic M: Pre-analytical effects of pneumatic tube transport on impedance platelet aggregometry. Platelets. 2009;20: 458-65.

12 Davenport RA, Brohi K: Coagulopathy in trauma patients: importance of thrombocyte function? Curr Opin Anaesthesiol. 2009;22: 261-6.

13 Apelseth TO, Bruserud O, Wentzel-Larsen T, Bakken AM, Bjorsvik S, Hervig T: In vitro evaluation of metabolic changes and residual platelet responsiveness in photochemical treated and gamma-irradiated single-donor platelet concentrates during long-term storage. Transfusion. 2007;47: 653-65.

14 Keuren JF, Cauwenberghs S, Heeremans J, de Kort W, Heemskerk JW, Curvers J: Platelet ADP response deteriorates in synthetic storage media. Transfusion. 2006;46: 204-12.

15 Heim D, Passweg J, Gregor M, Buser A, Theocharides A, Arber C, Meyer-Monard S, Halter J, Tichelli A, Gratwohl A: Patient and product factors affecting platelet transfusion results. Transfusion. 2008;48: 681-7.

Shanwell A, Diedrich B, Falker C, Jansson B, Sandgren P, Sundkvist L, Svensson L, Vesterinen M, Gulliksson H: Paired in vitro and in vivo comparison of apheresis platelet concentrates stored in platelet additive solution for 1 versus 7 days. Transfusion. 2006;46: 973-9.

EuropeanCouncil: The guide to the preparation, use and quality assurance of blood components. 16th edn: European Directorate for the Quality of Medicines \& Health Care; 2011.

18 Aursnes I, Sundal J, Nome T: Shear stress activation of platelets with subsequent refractoriness. Thromb Res. 1987;45: 29-37.

19 Albanyan AM, Harrison P, Murphy MF: Markers of platelet activation and apoptosis during storage of apheresis- and buffy coat-derived platelet concentrates for 7 days. Transfusion. 2009;49: 108-17.

20 Akay OM, Gunduz E, Basyigit H, Gulbas Z: Platelet function testing during 5-day storage of single and random donor plateletpheresis. Transfus Apher Sci. 2007;36: 285-9.

21 Javela K, Eronen J, Sarna S, Kekomaki R: Soluble glycoprotein V as a quality marker of platelet concentrates stressed by transportation. Transfusion. 2005;45: 1504-11.

22 Braun S, von Beckerath N, Ellert J, Kastrati A, Schomig A, Vogt W, Sibbing D: Assessment of platelet function in whole blood by multiple electrode aggregometry: transport of samples using a pneumatic tube system. Am J Clin Pathol. 2009;132: 802-3; author reply 3-4. 
23 Wallin O, Soderberg J, Grankvist K, Jonsson PA, Hultdin J: Preanalytical effects of pneumatic tube transport on routine haematology, coagulation parameters, platelet function and global coagulation. Clin Chem Lab Med. 2008;46: 1443-9.

24 Jambor C, Weber CF, Gerhardt K, Dietrich W, Spannagl M, Heindl B, Zwissler B: Whole blood multiple electrode aggregometry is a reliable point-of-care test of aspirin-induced platelet dysfunction. Anesth Analg. 2009;109: 25-31.

25 Toth O, Calatzis A, Penz S, Losonczy H, Siess W: Multiple electrode aggregometry: a new device to measure platelet aggregation in whole blood. Thromb Haemost. 2006;96: 781-8.

26 Dijkstra-Tiekstra MJ, Pietersz RN, Huijgens PC: Correlation between the extent of platelet activation in platelet concentrates and in vitro and in vivo parameters. Vox Sang. 2004;87: 257-63.

27 Rinder HM, Snyder EL, Tracey JB, Dincecco D, Wang C, Baril L, Rinder CS, Smith BR: Reversibility of severe metabolic stress in stored platelets after in vitro plasma rescue or in vivo transfusion: restoration of secretory function and maintenance of platelet survival. Transfusion. 2003;43: 1230-7.

28 Rank A, Nieuwland R, Liebhardt S, Iberer M, Grutzner S, Toth B, Pihusch R: Apheresis platelet concentrates contain platelet-derived and endothelial cell-derived microparticles. Vox Sang. 2011;100: 179-86.

29 Morel O, Morel N, Freyssinet JM, Toti F: Platelet microparticles and vascular cells interactions: a checkpoint between the haemostatic and thrombotic responses. Platelets. 2008;19: 9-23. 


\title{
Chapter 5a
}

\section{Obstetric hemorrhage and coagulation: an update}

Thromboelastography, thromboelastometry and conventional coagulation tests in the diagnosis and prediction of postpartum hemorrhage

Natascha M. de Lange, Marcus D. Lancé, Renée de Groot,

Erik A.M. Beckers, Yvonne M. Henskens, Hubertina C.J. Scheepers

\author{
Published in
}

Obstetrical \& Gynecological Survey

2012; 67(7):426-435 


\section{Abstract}

Globally, postpartum hemorrhage (PPH) is the leading cause of maternal morbidity and mortality. In current treatment of severe $\mathrm{PPH}$, first line therapy includes transfusion of packed cells and fresh frozen plasma in addition to uterotonic medical management and surgical interventions. In persistent $\mathrm{PPH}$, tranexamic acid, fibrinogen and coagulation factors are often administrated. Secondary coagulopathy due to PPH or its treatment is often underestimated and therefore remains untreated, potentially causing progression to even more severe $\mathrm{PPH}$. In most cases, medical and transfusion therapy is not based on the actual coagulative state, as conventional laboratory test results are usually not available for 45-60 minutes. Thromboelastography and rotational thromboelastometry are point of care coagulation tests. A good correlation is shown between thromboelastometric and conventional coagulation tests, and the use of these in massive bleeding in nonobstetric patients is widely practiced and has been proven to be cost effective. As with conventional laboratory tests, there is an influence of fluid dilution on coagulation tests that is more pronounced with colloid fluids. Fibrinogen seems to play a major role in the course of $\mathrm{PPH}$ and can be an early predictor in the severity of PPH. The FIBTEM values (in thromboelastometry, reagent specific for the fibrin polymerization process) decline even sooner than fibrinogen levels and might be used for early guidance of interventions. Data on thromboelastography and thromboelastometry in pregnant women are limited, particularly during the peripartum period and in women with $\mathrm{PPH}$, so more research in this field is needed. 


\section{Introduction}

Globally, obstetric hemorrhage is the leading cause of maternal morbidity and mortality, with approximately $25 \%$ of all maternal deaths caused by excessive blood loss and inadequate treatment or resources [1]. Berg et al. reported that $40 \%$ of the pregnancy-related deaths are potentially preventable, particularly deaths as a consequence of hemorrhage [2]. Obstetric hemorrhage currently causes $7 \%$ of maternal death in the Netherlands [3]. In addition to mortality, severe morbidity may occur after postpartum hemorrhage, i.e. loss of fertility, Sheehan's syndrome and multiple organ failure due to hypovolemic shock [4]. Taking these numbers and consequences into consideration, there is a need to improve the care around postpartum hemorrhage. The most common cause of massive postpartum hemorrhage is uterine atony; other causes include surgical incisions, lacerations or coagulation disorders. Abnormal placentation such as placenta preavia or placenta accreta are known risk factors for postpartum hemorrhage, the incidence in increasing mainly due the rising rate of caesarean section. Coagulation disorders may present as a primary or secondary cause of massive postpartum hemorrhage; the latter is often underestimated and undertreated. Secondary coagulation disorders may result from dilution (dilutional coagulopathy) or depletion of clotting factors or substrates such as erythrocytes or platelets (consumptive coagulopathy). The development of secondary coagulation disorders with delay in obstetric management are crucial prognostic factors for the severity of postpartum hemorrhage [5].

Many guidelines and flowcharts have been published to optimize the management of postpartum hemorrhage [6-9]. In these guidelines, conventional laboratory tests and vital parameters are used to guide transfusion. The use of thromboelastography (TEG) or thromboelastometry (ROTEM or TEM) in massive bleeding in non-obstetric patients is widely practiced and has been proven to be cost effective. Current guidelines for treatment of massive hemorrhage in trauma patients recommend the use of TEG or TEM [10-13]. However, TEG/TEM results in trauma patients cannot easily be compared with women during labor, as specific pregnancy and peripartum related hemodynamic and hemostatic changes are likely to influence TEG/TEM results. For correct interpretation of TEG/TEM in women with postpartum hemorrhage, normal reference values for pregnancy have yet to be determined. The aim of this narrative review is to discuss: hemostasis and hemostatic changes during pregnancy, definitions and management of postpartum hemorrhage, coagulation and 
postpartum hemorrhage, the principles of TEG and (RO)TEM and the use of these test systems in obstetric hemorrhage.

\section{Hemostasis and hemostatic changes during pregnancy}

Hemostasis is vital for preventing major blood loss as it seals sites of injury in the vascular system. However, it must be controlled to prevent blood from coagulating within the vasculature and restricting normal blood flow. In the last decades, the mechanism of the coagulation cascade has been revealed [14, 15]. It has been recognized that the intrinsic and extrinsic coagulation pathways are not redundant, but are parallel generators of factor Xa on different cell surfaces.

The cell-based model on coagulation consists of an initiation, amplification and propagation phase. It presumes tissue factor as sole initiator of coagulation. Tissue factor is expressed on cells outside the vascular system. Once an injury occurs, tissue factor-bearing cells are exposed to blood and circulating factor VIla immediately binds to tissue factor. This results in the generation of a small amounts of factor $\mathrm{Xa}$ and thrombin, that diffuse to platelets. In the amplification phase, thrombin leads to generation of activated forms of factors V, VIII and XI. In the propagation phase, the various coagulation factors assemble at the surface of activated platelets to form tenase and prothrombinase complexes, and thrombin generation. This leads to generation of fibrin from fibrinogen $[15,16]$.

During pregnancy, plasma volume increases by $40 \%$ and the erythrocyte count by $25 \%$. As a result, hemoglobin levels and hematocrit decrease due to this physiological hemodilution [17]. In addition, platelet levels drop due to hemodilution and increased consumption in the uteroplacental unit [17, 18]. In contrast, many coagulation factor levels increase during pregnancy, including von Willebrand factor, factor VII and VIII, as well as the levels of fibrinogen and factor IX. Other factors, such as factor XII and V remain unchanged [19, 20]. Natural coagulation inhibitors, such as protein $\mathrm{S}$, decrease while the fibrinolytic system is negatively influenced by increased plasminogen activator inhibitor (PAI) and thrombin-activatable fibrinolysis inhibitor (TAFI). Overall, these changes result in a state of hypercoagulability and hypofibrinolysis that is protective against excessive blood loss at delivery, but also leaves pregnant women more prone to suffer from thrombo-embolic events. Fibrinogen levels rise significantly from week 28 to double non-pregnant levels by term, and remain elevated throughout the first postpartum days [17, 18, 21-23]. The 
rise in fibrinogen is likely to be caused by an increase in synthesis due to hormonal changes, particularly elevated estrogen levels [24].

D-dimer values increase progressively during pregnancy, peaking at the first day postpartum and decreasing thereafter. This rise in D-dimer accompanies a simultaneous increase in circulating fibrinogen and other procoagulant factors during pregnancy. Despite hypofibrinolysis, the excess of fibrin deposition results via an increase in tissue plasminogen activator in higher D-dimer values [17-20, 24, 25]. Despite all these hemostatic alterations in pregnancy, laboratory studies show unchanged or only slightly decreased levels of activated partial thromboplastin time (aPTT), prothrombin time (PT) and international normalized ratio (INR); all of these remain within normal limits for non-pregnant women [20]. These overall hypercoaguable hemostatic changes contribute to the physiological preparation for delivery and prevention of excessive blood loss postpartum. An alteration in this equilibrium may influence the course of postpartum hemorrhage.

\section{Definitions and management of postpartum hemorrhage}

Definitions for postpartum hemorrhage vary. In general, postpartum hemorrhage is defined as blood loss of more than $500 \mathrm{~mL}$ within 24 hours after vaginal delivery or $1000 \mathrm{~mL}$ after caesarean section. Severe postpartum hemorrhage is indicates by blood loss of more than $1500 \mathrm{~mL}$, a decline in hemoglobin of more than $4 \mathrm{~g} / \mathrm{dL}$ or more, transfusion of at least four units of red blood cells or the need for a hemostatic (embolisation) or operative intervention. Massive blood loss is defined as the loss of one blood volume, the loss of $50 \%$ of blood volume within 3 hours or a loss rate of $150 \mathrm{~mL} /$ minute [26]. It is important to note that the amount of blood loss in postpartum hemorrhage is difficult to assess clinically and is usually underestimated $[27,28]$. The most common cause of PPH is uterine atony, which should be treated immediately by emptying the bladder and administering pharmacological agents such as oxytocin, ergometrine or misoprostol [7]. Other causes of blood loss, such as retained products of conception, uterine or birth canal trauma or pre-existing coagulation problems, must be considered as well.

Management should include the following steps: laboratory evaluation of hemoglobin level, hematocrit, platelet count, and fibrinogen concentration, as well as PT and aPTT. Volume resuscitation: initially fluid resuscitation, followed if necessary by transfusion of red blood cells to assure rapid correction of oxygen carrying 
capacity. The risk of dilutional coagulopathy due to fluid resuscitation should be considered. Correction of coagulation factors: depending on laboratory results and the clinical condition of the patient, fresh frozen plasma, fibrinogen, platelets or tranexamic acid can be administered. Off-label use of recombinant activated factor VII (Novo seven) should be considered only in refractory cases with ongoing bleeding after sufficient correction of all deficiencies has been achieved. Surgical measures include: evacuation of retained placental fragments, balloon tamponade, selective embolisation of the uterine artery, uterine compression sutures (B-lynch) or hysterectomy as necessary and clinically indicated [6-9]. It is important to note that massive postpartum hemorrhage is potentially life-threatening and requires a multidisciplinary approach, including all staff working at a labor ward, anesthesia and hematology. The Confidential Enquiry into Maternal and Child Health (CEMACH) strongly recommends the development of multidisciplinary massive hemorrhage guidelines, adapted to the hospital. Such protocols are already used in trauma medicine to simplify and expedite the delivery of blood products to hemorrhagic patients $[13,29,30]$.

In obstetrics there are few good publications of the implementation of massive hemorrhage guidelines in clinical practice [31-34]. There have been several publications on improved maternal outcome after the introduction of a multidisciplinary protocol and approach in postpartum hemorrhage, despite more cases of postpartum hemorrhage [35, 36]. The use of these protocols and regular local drill is recommended, as is attending the Managing Obstetric Emergency and Trauma (MOET) courses [7, 33].

\section{Coagulation factors in postpartum hemorrhage}

Primary coagulation disorders are not frequently identified as a cause of postpartum hemorrhage, except in women on anti-coagulation medication or with a known history of bleeding disorders [5]. Case series have shown a higher incidence of postpartum hemorrhage in women with inherited bleeding disorders such as von Willebrand disease (RR 1.5-3.0) [37-39]. Other causes that have been independently associated with a significant risk of severe postpartum hemorrhage include low levels of fibrinogen (even without reaching levels of deficiency), von Willebrand factor antigen, factor XI, platelet CD42b level, TRAP-induced increase of platelet CD41a expression, high values of serum residual prothrombin activity and blood group $O$. The presence 
of two or more independent risk factors showed an odds ratio of 16.4 for severe postpartum hemorrhage [40].

General screening of the pregnant population for disorders of coagulation would not be cost-effective nor informative due to low prevalence and insufficient data on reference ranges in pregnancy, as well as limited data on therapeutic algorithms based on such screening results. In women with clinical postpartum hemorrhage, however, primary coagulation disorders should be considered. Most patients with postpartum hemorrhage due to a coagulopathy will have a history that points to bleeding tendency (e.g. bruising, hematoma, bleeding with teeth brushing, and/or heavy menstrual bleeding) [41]. Severe postpartum hemorrhage may result in secondary coagulopathy, similar as seen during massive blood loss in trauma patients. This may be due to the consumption of coagulation factors, dilution, acidosis or hypothermia. Resuscitation with crystalloid fluids in large amounts may induce dilutional acidosis, formation of interstitial edema and impairment of microcirculation [42]. Colloid fluids, in particular synthetic colloids such as hydroxyethyl starch (HES) solutions may impair clot formation and therefore increase blood loss [43, 44]. Furthermore, even new generation medium molecular weight HES disturbs fibrin polymerization in patients undergoing spine surgery; the presence of HES or gelatin solutions in patients with fibrinolysis leads to faster clot disintegration $[45,46]$.

Progressive dilutional coagulopathy, hypothermia and metabolic acidosis have been referred to as the lethal triad in non-pregnant patients with massive blood loss $[47,48]$. In current guidelines for management of severe postpartum hemorrhage, transfusion of packed cells, fresh frozen plasma and platelets, in addition to uterotonic therapy, fluid resuscitation and surgical measures, is regarded as first line therapy [6-9]. In most cases, therapy of postpartum hemorrhage cannot be based on the actual hemostatic competence, as regular conventional coagulation tests typically require 45-60 minutes for results to be available and are non-specific [49]. One logistical challenge is the time needed to prepare plasma from anticoagulant whole blood samples. Another clinical challenge is that APTT (to detect deficiencies of coagulation factors VIII, IX, XI or XII) and PT (to detect deficiencies of coagulation factors $\mathrm{X}, \mathrm{VII}, \mathrm{V}$ or II) only measure the plasma component of the coagulation cascade. They both were initially developed as laboratory tests to monitor anticoagulant treatment, including heparin and vitamin $\mathrm{K}$ antagonists, respectively. 
Transfusion with fluids, packed red blood cells and FFP will aggravate dilutional coagulopathy, as has been demonstrated in cardiac surgery [50]. Such products do not correct coagulation disorders completely, as even FFP contains insufficient amounts of coagulation factors. Most postpartum hemorrhage protocols advocate the transfusion of 4 FFP for every 6 units of packed red cells or with a PT/aPTT >1.5 times normal, as do many guidelines for management of bleeding following major trauma.

In recent years there have been several publications that suggest a better outcome and fewer transfusions, when transfusing packed red blood cells and FFP on a 2:3 or even on a 1:1 ratio and early transfusion of FFP. The studies, however, are mainly retrospective $[13,51-53]$. In persistent $\mathrm{PPH}$, fibrinogen, coagulation factors and tranexamic acid are often administered. Tranexamic acid has an antifibrinolytic effect, and acts by blocking the conversion of plasminogen to plasma. It is effective in reducing peri- and post-operative blood loss $[54,55]$ and in reducing heavy menstrual bleeding [56]. During delivery of the placenta, there is rapid degradation of fibrinogen and fibrin, as well as activation of plasminogen activators and production of fibrin degradation products due to activation of the fibrinolytic system. The antifibrinolytic effect of tranexamic acid could theoretically reduce blood loss in the third stage of labor, although at present there is insufficient evidence to support its use in this setting [57]. Additionally, a study of Charbit et al. suggests that there is no evidence of hyperfibrinolysis in patients with PPH due to severe uterine atony [58]. The WOMAN Trial (World Maternal Antifibrinolytic Trial), a randomized controlled trial in which tranexamic acid is compared to placebo in women with $\mathrm{PPH}$, might resolve this issue [59].

The off-label use of pro-coagulative agents such as recombinant factor VIla is only indicated and effective in the presence of adequate substrate for coagulation (fibrinogen and platelets) and is not without risk, a meta-analysis reported significantly more arterial thrombotic events in patents receiving recombinant factor VIla [60,61]. Possible side effects of uncontrolled administration of blood products and factor concentrates also include thrombosis and volume overload. The absence of quick and specific laboratory tests to monitor postpartum hemorrhage and assess the development of a secondary coagulopathy can result in excessive and avoidable usage of blood products. A preferred approach would be the selective administration of blood products and clotting factors according to depletion [62, 63]. The general 
protocols and guidelines utilized for the non-pregnant patient are likely not adequate or appropriate for peripartum women.

In pregnancy, the relative hypercoagulable state makes uncontrolled treatment potentially more dangerous. In addition, there are no clear therapeutic thresholds defined for peripartum women. It is unclear whether treatment should be aimed at achieving minimal levels described for non-pregnant subjects or whether pregnancy normal values should be the goal. Evaluation of fibrinogen levels presents a good example of this issue; Charbit et al. [58] investigated different coagulation parameters during PPH and found that PT, INR, aPTT, fibrinogen, D-dimer, prothrombin and factor $\mathrm{V}$ were significantly lower in a group of women with severe postpartum hemorrhage compared to women with milder postpartum hemorrhage. In both groups, however, the fibrinogen values were within normal range for non-pregnant subjects ( 2 to $4 \mathrm{~g} / \mathrm{L}$ ). In the peripartum period, however, normal fibrinogen values are above what is considered normal and vary from 3.5 to $6.5 \mathrm{~g} / \mathrm{L}$ [20]. Consequently, values used to guide interventions might differ from non-pregnant levels. Fibrinogen is of importance in forming a firm clot and is the first parameter to decrease during massive bleeding, due to increased fibrinogen consumption as well as hemodilution resulting from fluid administration. Fibrinogen is the only parameter that has been shown to be significantly lower and independently associated with progress toward severe bleeding. Fibrinogen levels $\leq 2 \mathrm{~g} / \mathrm{L}$ have been reported to have a positive predictive value of $100 \%$ and therefore could be an early predictor of severe postpartum hemorrhage [58]. Gayat et al. published a retrospective cohort on predictors of severe postpartum hemorrhage; in a univariate analysis fibrinogen levels $<2 \mathrm{~g} / \mathrm{L}$ and prothrombin time $<50 \%$ were significant preditors of severe post partum hemorrhage, while in the multivariate analysis only fibrinogen levels $<2 \mathrm{~g} / \mathrm{L}$ remained significant [64]. Bell and co-workers presented 6 cases of obstetric hemorrhage associated with hypofibrinogenemia (all fibrinogen levels $<1.5 \mathrm{~g} / \mathrm{L}$ ), who were treated with fibrinogen. All coagulation tests rapidly normalized and severe hemorrhage improved. Notably, however, all patients also received platelets, fresh frozen plasma and packed red cells [65]. In contrast to obstetrics, many surgical specialties utilize early administration of fibrinogen in cases of severe bleeding. Although data are limited, results consistently favor the early administration of fibrinogen over FFP in bleeding patients [66]. 


\section{Principles of thromboelastography (TEG) and rotational thromboelastometry (ROTEM)}

Thromboelastography and rotational thromboelastometry are highly similar, dynamic, visco-elastic coagulation tests, which encompass the whole coagulation process from the initiation of coagulation to clot formation and fibrinolysis. The tests can be performed in a point-of-care setting, with whole blood instead of plasma. Devices in which these tests are performed allow analyzing multiple samples at the same time.

The technique of thromboelastography was first introduced by Hartert in 1948. Clinically, it was used in 1985 to check hemostatic activity during hepatic and cardiothoracic surgery. The current thromboelastograph (TEG) device consists of a plastic pin attached to a torsion wire that is immersed in a rotating cup. During the process of clot formation, an electrical signal produces a time cuve of the elasticity of the formed clot (Figure 1). The outcome of a TEG tracing is presented as five parameters:

- $\mathrm{R}$ : the time from starting the test to that of initial fibrin formation, determined by the curve slope (seconds)

- $\mathrm{K}$ : the time from initial fibrin formation till an amplitude is reached of $20 \mathrm{~mm}$ (seconds)

- $\alpha$ angle: the maximal slope of the curve $\left({ }^{\circ}\right)$

- maximal amplitude (MA) of the fibrin formation curve (mm)

- Ly30: the extent of amplitude reduction at 30 minutes after MA.

Alterations in the normal TEG curve and in the different curve parameters appear to provide useful information regarding abnormalities in the coagulation process. A prolonged $\mathrm{R}$ value points to delayed coagulation factor activation; the $\mathrm{K}$ and $\alpha$ angle are parameters of coagulation factor amplification; the MA reflects platelet function, and the Ly30 detects fibrinolysis activity.

Tests with this assay can also be run in native whole blood. However, it is common to use anticoagulated blood with coagulation activators to stimulate different pathways. Tissue factor/ $\mathrm{CaCl}_{2}$ or kaolin/ $\mathrm{CaCl}_{2}$ are added to trigger the extrinsic or intrinsic coagulation pathways, respectively. If required, heparinase and platelet GPIIb/Illa inhibitors can be added to provide more detailed information in specific clinical settings [67]. 

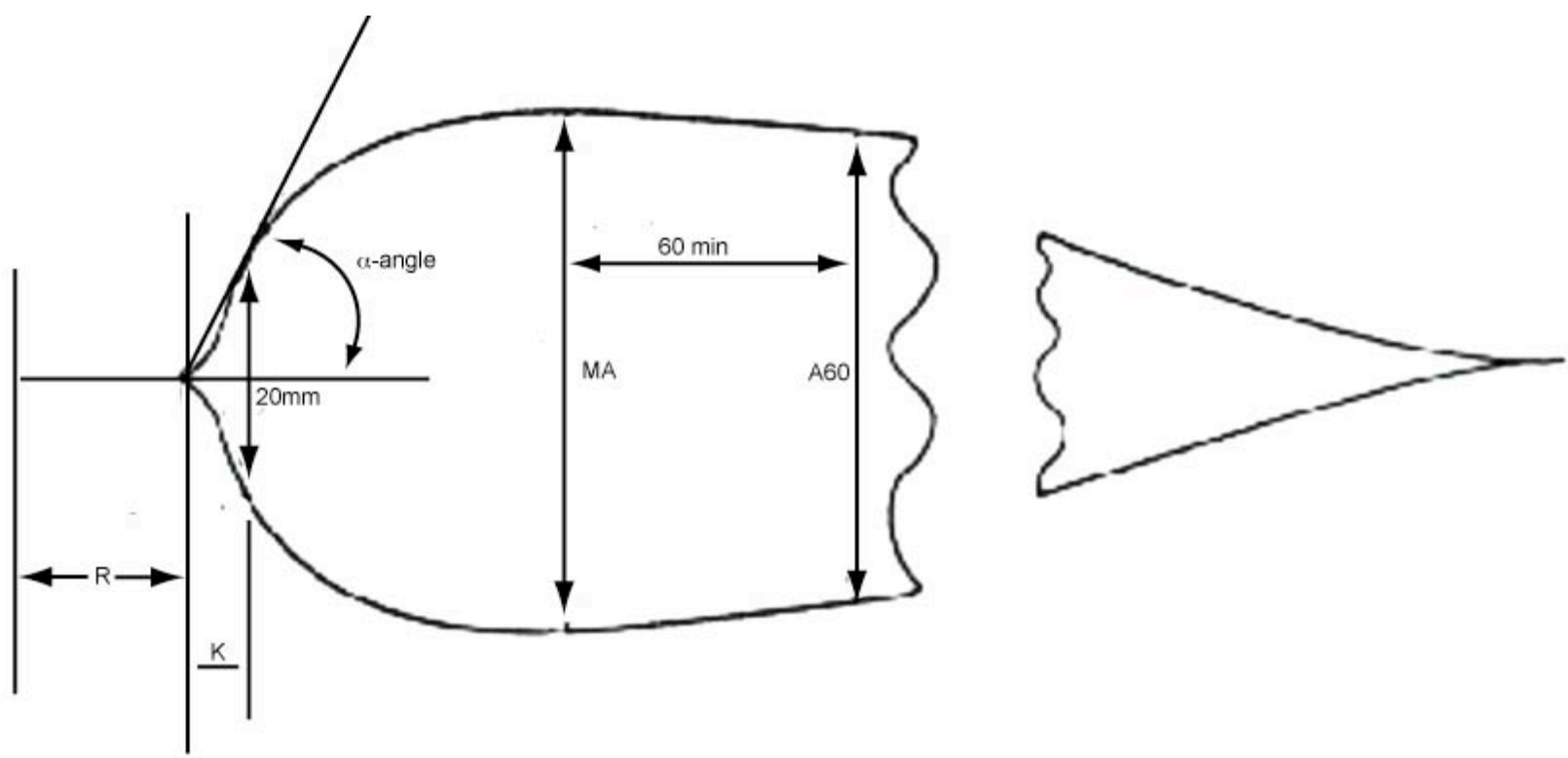

Figure 1: Parameters of thromboelastography (TEG) in a graph. For explanation, see text.

Rotatonional thromboelastometry (ROTEM) is based on the same principles as thromboelastography, but now a rotating plastic pin fixed on a steel axis is immersed into a cup that is not moving. The movement of the pin is converted to an optical signal and transferred to a graphical display (Figure 2). The outcome of a ROTEM tracing is very similar to that of TEG with results, again presented as five parameters:

- CT (clotting time): time from start of measurement until initiation of clotting (seconds)

- CFT (clot formation time): time from initiation of the clotting until a curve amplitude of $20 \mathrm{~mm}$ is detected (seconds)

- $\alpha$ angle: angle of the maximal slope of the curve $\left(^{\circ}\right)$

- MCF (maximum clot firmness): maximal amplitude reached in the curve ( $\mathrm{mm})$

- ML (maximum lysis): reduction of the clot firmness (MCF) as a function the $\operatorname{MCF}(\%)$.

Also in this case of the ROTEM test, different coagulation activators are used to initiate the coagulation process. In the so-called INTEM assay, $\mathrm{CaCl}_{2}$, phospholipids and ellagic acid are used to activate the intrinsic pathway. In the EXTEM assay, tissue factor is used for activation of the extrinsic pathway. In the FIBTEM assay a platelet inhibitor (cytochalasin D) is added to the blood sample, and tissue factor plus $\mathrm{CaCl}_{2}$ are used to trigger. This test differentiates between platelet dysfunction and 


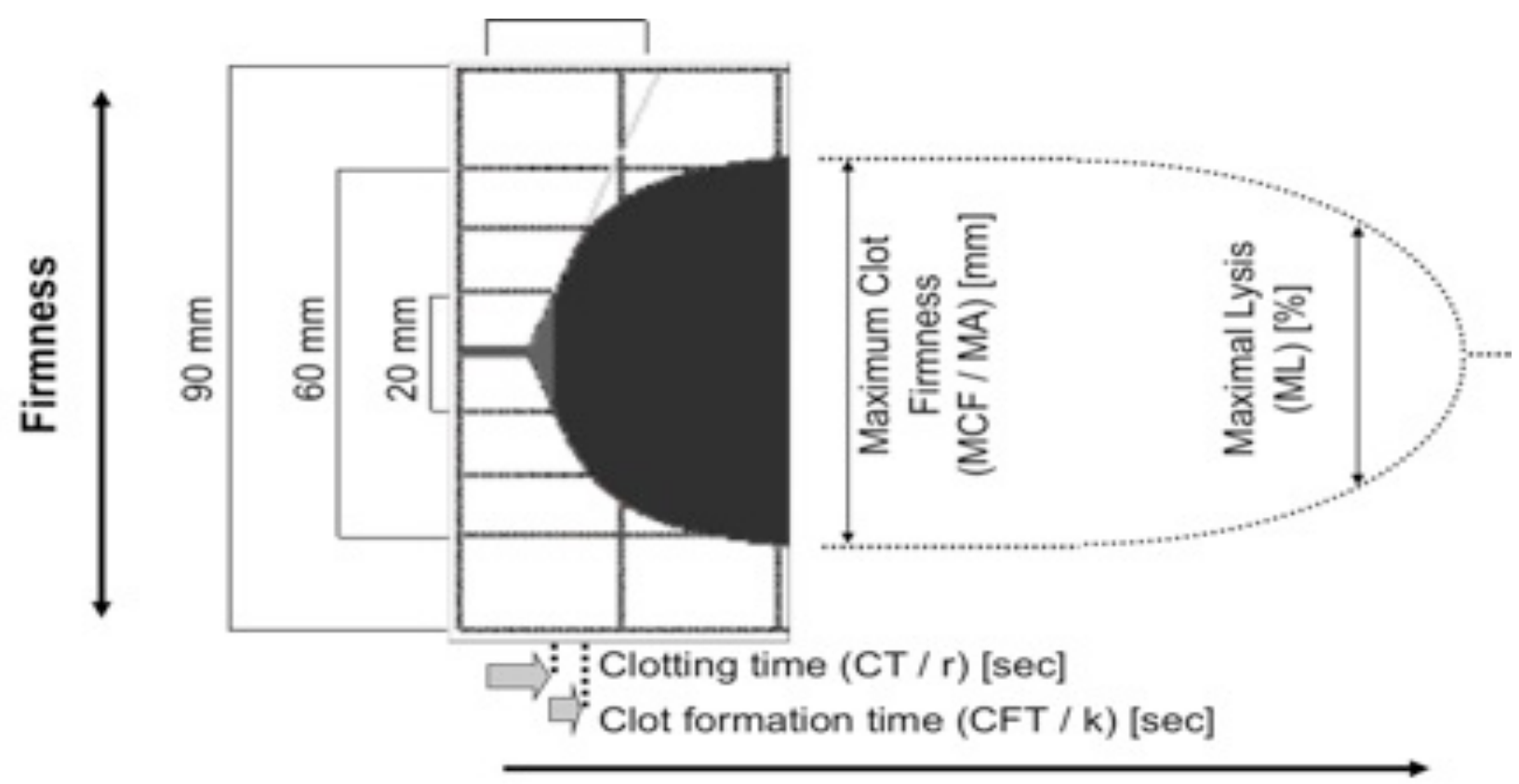

Figure 2: Parameters of rotational thromboelastometry (ROTEM) in a graph. For explanation, see text.

impaired fibrin formation. In the APTEM assay, a fibrinolysis inhibitor (aprotinin) is used together with tissue factor and $\mathrm{CaCl}_{2}$ to check for hyperfibrinolysis. Abnormalities are further distinguished in the following ways. In case of initial clotting abnormality due to coagulation factor deficiency: the clotting time (CT) and clot formation time (CFT) are prolonged and the angle of the curve is reduced. Specific defiencies in the extrinsic or intrinsic coagulation pathways are detected by comparing the EXTEM and INTEM assays. In case of fibrinogen deficiency: the CT is normal, the CFT is slightly prolonged and the maximum clot firmness (MCF) is reduced. Platelets are non-functional in FIBTEM assay, implying that the test evaluates a specific lowering in fibrinogen level. Using the APTEM test in combination with the EXTEM test, the extent of fibrinolysis can be determined [12, $68,69]$.

\section{Use of thromboelastography and rotational thromboelastometry in obstetrics}

Several publications discuss the use of these clot formation tests, but with little attention to its use in pregnancy. Limited studies have compared blood parameters of pregnant women to non-pregnant subjects, and their confirmed hypercoaguable state of parturients with thromboelastography [70,71]. Other studies focused on specific situations during pregnancy and delivery. A short overview is given in the following. 


\section{Comparing pregnant with non pregnant women}

Armstrong and colleagues compared the ROTEM parameters of clot formation for blood from healthy pregnant women and healthy, non-pregnant female controls. They confirmed the presence of a hypercoaguable state in normal pregnancy, and conclude that this point-of-care test can be a helpful adjunct to conventional laboratory testing, because it reduces delays in instituting appropriate treatment. Reference values for pregnant women have not been determined [72]. Huissoud et al. showed a significant increase in clot amplitude at 5 and 15 seconds (INTEM, EXTEM and FIBTEM) for healthy pregnant women in the second and third trimester. This is again in concordance with a hypercoaguable state of pregnancy. The clotting time and clot lysis index at 30 minutes did not change during pregnancy. Furthermore, a significant correlation was obtained between ROTEM and conventional coagulation tests, e.g. a correlation between fibrinogen concentration and clot amplitude in the FIBTEM analysis was found [73]. These data combined with the results of Charbit and colleagues suggested that thromboelastography can be used as a rapid diagnostic tool to detect a decrease in fibrinogen in cases of severe bleeding [58].

Oudghiri and colleagues [74] tried to define reference values for ROTEM parameters in blood from a small group of women in the postpartum period after uncomplicated deliveries (without PPH). A strong correlation between thromboelastometric and conventional coagulation parameters was again found, especially regarding fibrinogen concentration and the FIBTEM assay. Unfortunately, the study excluded women with postpartum hemorrhage, and had a small sample size.

\section{Hemodilution}

The effect of hemodilution on thromboelastometric parameters has been studied in pregnant women, e.g. after elective caesarean section to detect peripartal changes and blood loss. Butwick et al. [75] demonstrated that preloading with hydroxyethyl starch (HES, $500 \mathrm{~mL}$ ) but not lactated Ringer solution $(1500 \mathrm{~mL})$ resulted in mild coagulation effects in the clot formation time measured with TEG. Turker et al. showed a mild hypocoaguable effect on TEG parameters (shorter R-time and lower maximum amplitude) in healthy parturients after preload with HES or succinylated gelatine before elective caesarean section. Ansari and co-workers demonstrated that 
in vitro dilution to $60 \%$ with lactated Ringer solution in the presence or absence of HES caused changes in all clot formation parameters, measured by ROTEM [76, 77].

\section{Use of thromboelastography or thromboelastometry in obstetric hemorrhage}

A number of studies have assessed the utility of TEG or ROTEM testing in relation to pregnancy. Butwick assessed the correlation between TEG parameters and blood loss in elective caesarean section. They show a weak association between clot strength (maximum amplitude) and blood loss, and a modest reduction in maternal hypercoagulability during the early postpartum period [78]. In a prospective observational study focused on postpartum hemorrhage, the group of Huissoud compared 51 women with hemorrhage to a historical control group, and showed that in ROTEM, the FIBTEM-derived clotting time was significantly prolonged and clot amplitudes were significantly lower in the postpartum hemorrhage group compared to the non-bleeding control group [79]. However, the authors to no inform on the moment of blood sampling and the amount of blood loss. A strong correlation between fibrinogen level and FIBTEM assay parameters was present in the postpartum hemorrhage and the control group.

In an unpublished study by Ducloy-Bouters (expert meeting, Munich, April 2010), the cut-off values for the FIBTEM assay were evaluated at 30 minutes postpartum in a small number of women (12 PPH and 30 controls) [80]. Low fibrinogen levels $(<4 \mathrm{~g} / \mathrm{L})$ and a low maximum clot firmness $(<19 \mathrm{~mm})$ were seen in 10 out of 12 patients in the postpartum hemorrhage group, but in only 5 out of 30 patients of the control group. It was concluded that these values inform on the risk of bleeding in postpartum hemorrhage. Another small study by Bauters et al. compared results from 23 patients with postpartum hemorrhage and 31 women without hemorrhage [81]. The authors defined postpartum hemorrhage as a blood loss $>800$ $\mathrm{mL}$ and analyzed the first blood sample at the moment of diagnosis, and another sample two hours later. In patients with postpartum hemorrhage, a decrease in FIBTEM values was observed already before major decreases in plasma fibrinogen, suggesting coagulopathy and a subsequent fibrinogen decrease. There are no other studies are available with postpartum hemorrhage patients, which hampers routine use in this clinical setting. 


\section{Limitations}

As with any laboratory test, TEG and ROTEM assays have limitations. Adequate training is required and the inclusion of quality control samples. Furthermore, the tests are of limited use with blood from patients on platelet inhibitors, as platelets contribute to the measured clot formation amplitude [82]. In some cases, it has not been possible to discriminate between thrombocytopenia or disorders of platelet function [83, 84]. Treatment with fibrinogen may increase maximal clot firmness in INTEM and EXTEM and disguise thrombocytopenia [85].

Another limitation is the measurement temperature: TEG and ROTEM analysis are routinely performed at $37^{\circ} \mathrm{C}$. As a consequence of hypothermia is not recognized and may be left untreated [86, 87]. Finally, fibrinogen is a well-known acute phase protein, which can also be important in cases of $\mathrm{PPH}$.

\section{Conclusions}

Conventional coagulation tests are time-consuming in acute situations and cannot predict the course of massive blood loss. As a consequence, in many cases blood products are given in an uncontrolled way, which is risky and expensive. In contrast, TEG and ROTEM to measure elastic clot formation in whole blood are rapid tests that can help to differentiate potential causes of hemostatic failure. The outcome of both tests correlates well with the plasma fibrinogen level. As with conventional laboratory tests, there is an influence of fluid dilution on coagulation test results that is more pronounced when colloid fluids are administered. Low fibrinogen levels can be detected earlier with the FIBTEM assay than with conventional tests in women with post partum hemorrhage. While there are some data regarding the use of TEG and ROTEM in blood from pregnant women, normal reference values are lacking and cut off values have not been defined.

The evidence available at this moment does not support the use of TEG or ROTEM to screen for coagulation disorders in healthy women to predict postpartum hemorrhage. However, in secondary coagulopathy as a consequence of postpartum hemorrhage they can help in early diagnosis and treatment, given the role of fibrinogen in postpartum hemorrhage. Future research should be directed to establish: (i) normal values for TEG/ROTEM in uncomplicated deliveries, (ii) cut-off values for intervention to determine whether treatment should start at certain FIBTEM parameters (correlating with fibrinogen levels) for (non-)pregnant women; and (iii) 
cost-effectiveness of TEG/ROTEM based care compared to current care in postpartum hemorrhage.

\section{References}

1 The world health report 2005 - Make every mother and child count. Geneva: World Health Organisation 2005.

2 Berg C, Harper M, Atkinson S, Bell E, Brown H, Hage M, Mitra A, Moise Kj, Callaghan W: Preventability of pregnancy-related deaths: results of a state-wide review. Obstet Gynecol. 2005;106: 1228-34.

Schutte J, Steegers E, Schuitemaker N, Santema J, Boer Kd, Pel M, Vermeulen G, Visser W, Roosmalen Jv, Committee tNMM: Rise in maternal mortality in the Netherlands. Br J Obstet Gynaecol. 2010;117: 399-406.

4 Gynecologists ACoOa: ACOG Practice bulletin: clinical management guidelines for obstetrician-gynecologists. Obstet Gynecol. 2006;108: 1039-47.

5 McLintock C: Obstetric haemorrhage. Thromb Res. 2009;123 Suppl 2: S30-4.

6 Leduc D, Senikas V, Lalonde AB, Ballerman C, Biringer A, Delaney M, Duperron L, Girard I, Jones D, Lee LS, Shepherd D, Wilson K: Active management of the third stage of labour: prevention and treatment of postpartum hemorrhage. J Obstet Gynaecol Can. 2009;31: 98093.

7 Wise A, Clark V: Strategies to manage major obstetric haemorrhage. Curr Opin Anaesthesiol. 2008;21: 281-7.

8 Ahonen J, Stefanovic V, Lassila R: Management of post-partum haemorrhage. Acta Anaesthesiol Scand.54: 1164-78.

9 Knight M, Callaghan WM, Berg C, Alexander S, Bouvier-Colle MH, Ford JB, Joseph KS, Lewis $\mathrm{G}$, Liston RM, Roberts CL, Oats J, Walker J: Trends in postpartum hemorrhage in high resource countries: a review and recommendations from the International Postpartum Hemorrhage Collaborative Group. BMC Pregnancy Childbirth. 2009;9: 55.

10 Theusinger OM, Spahn DR, Ganter MT: Transfusion in trauma: why and how should we change our current practice? Curr Opin Anaesthesiol. 2009;22: 305-12.

Spalding G, Hartrumpf M, Sierig T, Oesberg N, Kirschke C, Albes J: Cost reduction of perioperative coagulation management in cardiac surgery: value of 'bedside' thrombelastography (ROTEM). Eur J Cardiothorac Surg. 2007;31: 1052-7.

12 Innerhofer P, Streif W, Kuhbacher G, Fries D: Monitoring of perioperative dilutional coagulopathy using the ROTEM analyzer: basic principles and clinical examples. Anasthesiol Intensivmed Notfallmed Schmerzther. 2004;39: 739-44.

13 Rossaint R, Bouillon B, Cerny V, Coats TJ, Duranteau J, Fernandez-Mondejar E, Hunt BJ, Komadina R, Nardi G, Neugebauer E, Ozier Y, Riddez L, Schultz A, Stahel PF, Vincent JL, Spahn DR: Management of bleeding following major trauma: an updated European guideline. Crit Care. 2010;14: R52.

14 Hoffman M, Monroe DM: Coagulation 2006: a modern view of hemostasis. Hematol Oncol Clin North Am. 2007;21: 1-11.

15 Hoffman M: Remodeling the blood coagulation cascade. J Thromb Thrombolysis. 2003;16: 1720.

16 Smith SA: The cell-based model of coagulation. J Vet Emerg Crit Care (San Antonio). 2009;19: 3-10.

17 Abbassi-Ghanavati M, Greer LG, Cunningham FG: Pregnancy and laboratory studies: a reference table for clinicians. Obstet Gynecol. 2009;114: 1326-31.

18 Cerneca F, Ricci G, Simeone R, Malisano M, Alberico S, Guaschino S: Coagulation and fibrinolysis changes in normal pregnancy. Increased levels of procoagulants and reduced levels of inhibitors during pregnancy induce a hypercoagulable state, combined with a reactive fibrinolysis. Eur J Obstet Gynecol Reprod Biol. 1997;73: 31-6.

19 Franchini M: [Post-partum hemorrhage: new therapeutic options]. Recenti Prog Med. 2007;98: 7-11.

20 Szecsi PB, Jorgensen M, Klajnbard A, Andersen MR, Colov NP, Stender S: Haemostatic reference intervals in pregnancy. Thromb Haemost. 2010;103: 718-27.

21 Choi J, Pai S: Tissue plasminogen activator levels change with plasma fibrinogen concentrations during pregnancy. Ann Hematol. 2002;81: 611-5. 
Francalanci I, Comeglio P, Liotta AA, Cellai AP, Fedi S, Parretti E, Mello G, Prisco D, Abbate R: D-dimer concentrations during normal pregnancy, as measured by ELISA. Thromb Res. 1995;78: 399-405.

23 O'Riordan MN, Higgins JR: Haemostasis in normal and abnormal pregnancy. Best Pract Res Clin Obstet Gynaecol. 2003;17: 385-96.

24 Francalanci I, Comeglio P, Liotta A, Cellai A, Fedi S, Parretti E: D-dimer concentrations durig normal pregnancy, as measured by ELISA. Thromb Res. 1995;78: 399-405.

25 Kline J, Williams G, Hernandez-Nino J: D-dimer concentrations in normal pregnancy: new diagnostic thresholds are needed. Clin Chem. 2005;51: 825-9.

26 MacPhail S. TK: Massive post-partum haemorrhage and management of disseminated intravascular coagulation. Curr Obstet Gynaecol. 2004;14: 123-31.

27 Hamilton-Davies C, Mythen MG, Salmon JB, Jacobson D, Shukla A, Webb AR: Comparison of commonly used clinical indicators of hypovolaemia with gastrointestinal tonometry. Int Care Med. 1997;23: 276-81.

28 Stafford I, Dildy GA, Clark SL, Belfort MA: Visually estimated and calculated blood loss in vaginal and cesarean delivery. Am J Obstet Gynecol. 2008;199: 519 e1-7.

29 Johansson PI, Ostrowski SR, Secher NH: Management of major blood loss: an update. Acta Anaesthesiol Scand. 2010;54: 1039-49.

30 Spahn DR, Cerny V, Coats TJ, Duranteau J, Fernandez-Mondejar E, Gordini G, Stahel PF, Hunt BJ, Komadina R, Neugebauer E, Ozier Y, Riddez L, Schultz A, Vincent JL, Rossaint R: Management of bleeding following major trauma: a European guideline. Crit Care. 2007;11: R17.

31 Burtelow M, Riley E, Druzin M, Fontaine M, Viele M, Goodnough LT: How we treat: management of life-threatening primary postpartum hemorrhage with a standardized massive transfusion protocol. Transfusion. 2007;47: 1564-72.

32 Merien AE, van de Ven J, Mol BW, Houterman S, Oei SG: Multidisciplinary team training in a simulation setting for acute obstetric emergencies: a systematic review. Obstet Gynecol. 2010;115: 1021-31.

33 Howell C, Grady K, Cox C: The MOET Course Manual: Managing Obstetric Emergencies and Trauma. Second edn: RCOG Press; 2009.

34 Al Kadri HM: Obstetric medical emergency teams are a step forward in maternal safety! J Emerg Trauma Shock. 2010;3: 337-41.

35 Skupski DW, Lowenwirt IP, Weinbaum FI, Brodsky D, Danek M, Eglinton GS: Improving hospital systems for the care of women with major obstetric hemorrhage. Obstet Gynecol. 2006;107: 977-83.

36 Varatharajan L, Chandraharan E, Sutton J, Lowe V, Arulkumaran S: Outcome of the management of massive postpartum hemorrhage using the algorithm "HEMOSTASIS". Int $J$ Gynaecol Obstet. 2011;113: 152-4.

37 James $\mathrm{AH}$ : More than menorrhagia: a review of the obstetric and gynaecological manifestations of bleeding disorders. Haemophilia. 2005;11: 295-307.

38 James $\mathrm{AH}$, Jamison MG: Bleeding events and other complications during pregnancy and childbirth in women with von Willebrand disease. J Thromb Haemost. 2007;5: 1165-9.

Al-Zirqi I, Vangen S, Forsen L, Stray-Pedersen B: Prevalence and risk factors of severe obstetric haemorrhage. BJOG. 2008;115: 1265-72.

40 Chauleur C, Cochery-Nouvellon E, Mercier E, Aya G, Fabbro-Peray P, Mismetti P, LissadeLavigne G, Gris JC: Some hemostasis variables at the end of the population distributions are risk factors for severe postpartum hemorrhages. J Thromb Haemost. 2008;6: 2067-74.

41 Chee YL, Crawford JC, Watson HG, Greaves M: Guidelines on the assessment of bleeding risk prior to surgery or invasive procedures. British Committee for Standards in Haematology. Br J Haematol. 2008;140: 496-504.

42 Fries D IP, Schobersberger W: Time for changing coagulation management in trauma-related massive bleeding. Curr Opin Anaesthesiol. 2009;22: 267-74.

43 Kozek-Langenecker SA: Effects of hydroxyethyl starch solutions on hemostasis. Anesthesiology. 2005;103: 654-60.

44 Westphal M JM, Kozek-Langenecker S, Stocker R, Guidet P, Van Aken H: Hydoxylethyl starches. Anesthesiology. 2009;111: 187-202.

45 Mittermayr M SW, Haas T, Fries D, Velik-Salchner C, Klingler A, Oswald E, Bach C, Schnapka-Koepf M, Innerhofer P.: Hemostatic changes after crystalloid od colloid fluid administration during major orthopedic surgery: the role of fibrinogen administration. Anesth Analg. 2007;105: 905-17. 
Mittermayr M SW, Haas T, Fries D, Velik-Salchner C, Klingler A, Innerhofer P.: Effect of colloid and crystalloid solutions on endogenous activation of fibrinolysis and resistance of polymerized fibrin to recombinant tissue plasmingen activator added ex vivo. $\mathrm{Br} \mathrm{J}$ Anaesth. 2008;100: 307-14.

47 Spahn DR, Rossaint R: Coagulopathy and blood component transfusion in trauma. $\mathrm{Br} \mathrm{J}$ Anaesth. 2005;95: 130-9.

48 Cosgriff N, Moore EE, Sauaia A, Kenny-Moynihan M, Burch JM, Galloway B: Predicting lifethreatening coagulopathy in the massively transfused trauma patient: hypothermia and acidoses revisited. J Trauma. 1997;42: 857-61.

49 Kitchens CS: To bleed or not to bleed? Is that the question for the PTT? J Thromb Haemost. 2005;3: 2607-11.

50 Schols SE, Lance MD, Feijge MA, Damoiseaux J, Marcus MA, Hamulyak K, Ten Cate H, Heemskerk JW, van Pampus EC: Impaired thrombin generation and fibrin clot formation in patients with dilutional coagulopathy during major surgery. Thromb Haemost. 2010;103: 31828.

51 Zink KA, Sambasivan CN, Holcomb JB, Chisholm G, Schreiber MA: A high ratio of plasma and platelets to packed red blood cells in the first 6 hours of massive transfusion improves outcomes in a large multicenter study. Am J Surg. 2009;197: 565-70.

Duchesne JC, Islam TM, Stuke L, Timmer JR, Barbeau JM, Marr AB, Hunt JP, Dellavolpe JD, Wahl G, Greiffenstein P, Steeb GE, McGinness C, Baker CC, McSwain NE, Jr.: Hemostatic resuscitation during surgery improves survival in patients with traumatic-induced coagulopathy. J Trauma. 2009;67: 33-7.

53 Duchesne JC, Hunt JP, Wahl G, Marr AB, Wang YZ, Weintraub SE, Wright MJ, McSwain NE, Jr.: Review of current blood transfusions strategies in a mature level I trauma center: were we wrong for the last 60 years? J Trauma. 2008;65: 272-6.

54 Reid RW, Zimmerman AA, Laussen PC, Mayer JE, Gorlin JB, Burrows FA: The efficacy of tranexamic acid versus placebo in decreasing blood loss in pediatric patients undergoing repeat cardiac surgery. Anesth Analg. 1997;84: 990-6.

55 Dryden PJ, O'Connor JP, Jamieson WR, Reid I, Ansley D, Sadeghi H, Burr LH, Munro Al, Merrick PM: Tranexamic acid reduces blood loss and transfusion in reoperative cardiac surgery. Can J Anaesth. 1997;44: 934-41.

56 Lethaby A, Farquhar C, Cooke I: Antifibrinolytics for heavy menstrual bleeding. Cochrane Database Syst Rev. 2000: CD000249.

57 Novikova N, Hofmeyr GJ: Tranexamic acid for preventing postpartum haemorrhage. Cochrane Database Syst Rev. 2010: CD007872.

58 Charbit B, Mandelbrot L, Samain E, Baron G, Haddaoui B, Keita H, Sibony O, Mahieu-Caputo D, Hurtaud-Roux MF, Huisse MG, Denninger MH, de Prost D: The decrease of fibrinogen is an early predictor of the severity of postpartum hemorrhage. J Thromb Haemost. 2007;5: 26673.

59 Shakur H, Elbourne D, Gulmezoglu M, Alfirevic Z, Ronsmans C, Allen E, Roberts I: The WOMAN Trial (World Maternal Antifibrinolytic Trial): tranexamic acid for the treatment of postpartum haemorrhage: an international randomised, double blind placebo controlled trial. Trials. 2010;11: 40.

60 Mercier FJ, Bonnet MP: Use of clotting factors and other prohemostatic drugs for obstetric hemorrhage. Curr Opin Anaesthesiol. 2010;23: 310-6.

61 Levi M, Levy JH, Andersen HF, Truloff D: Safety of recombinant activated factor VII in randomized clinical trials. N Engl J Med. 2010;363: 1791-800.

62 Pacheco LD, Saade GR, Gei AF, Hankins GD: Cutting-edge advances in the medical management of obstetrical hemorrhage. Am J Obstet Gynecol. 2011.

63 Ickx BE: Fluid and blood transfusion management in obstetrics. Eur J Anaesthesiol. 2010;27: 1031-5.

64 Gayat E, Resche-Rigon M, Morel O, Rossignol M, Mantz J, Nicolas-Robin A, Nathan-Denizot $\mathrm{N}$, Lefrant JY, Mercier FJ, Samain E, Fargeaudou Y, Barranger E, Laisne MJ, Brechat PH, Luton D, Ouanounou I, Plaza PA, Broche C, Payen D, Mebazaa A: Predictive factors of advanced interventional procedures in a multicentre severe postpartum haemorrhage study. Intensive Care Med. 37: 1816-25.

65 Bell SF, Rayment R, Collins PW, Collis RE: The use of fibrinogen concentrate to correct hypofibrinogenaemia rapidly during obstetric haemorrhage. Int J Obstet Anesth.19: 218-23. plasma compared with fibrinogen concentrate: a systematic review. Crit Care. 2011;15: R239. 
Luddington RJ: Thrombelastography/thromboelastometry. Clin Lab Haematol. 2005;27: 81-90. http://www.rotem.de/

Polak F, Lips M, Bedrichova $H$, Kriz P, Parizek A: [The use of thrombelastography in evaluation of coagulation in females with physiological or pathological gravidity]. Cas Lek Cesk. 2007;146: 396-400.

71 Sharma SK, Philip J, Wiley J: Thromboelastographic changes in healthy parturients and postpartum women. Anesth Analg. 1997;85: 94-8.

72 Armstrong S, Fernando R, Ashpole K, Simons R, Columb M: Assessment of coagulation in the obstetric population using ROTEM(R) thromboelastometry. Int J Obstet Anesth. 20: 293-8. Huissoud C, Carrabin N, Benchaib M, Fontaine O, Levrat A, Massignon D, Touzet S, Rudigoz $\mathrm{RC}$, Berland M: Coagulation assessment by rotation thrombelastometry in normal pregnancy. Thromb Haemost. 2009;101: 755-61.

74 Oudghiri M, Keita H, Kouamou E, Boutonnet M, Orsini M, Desconclois C, Mandelbrot L, Daures JP, Stepanian A, Peynaud-Debayle E, de Prost D: Reference values for rotation thromboelastometry $(\operatorname{ROTEM}(\mathrm{R}))$ parameters following non-haemorrhagic deliveries. Correlations with standard haemostasis parameters. Thromb Haemost. 2011;106: 176-8.

75 Butwick A, Carvalho B: The effect of colloid and crystalloid preloading on thromboelastography prior to Cesarean delivery. Can J Anaesth. 2007;54: 190-5.

76 Turker G, Yilmazlar T, Mogol EB, Gurbet A, Dizman S, Gunay H: The effects of colloid preloading on thromboelastography prior to caesarean delivery: hydroxyethyl starch 130/0.4 versus succinylated gelatine. J Int Med Res. 2011;39: 143-9.

77 Ansari T, Riad W: The effect of haemodilution with $6 \%$ hydroxyethyl starch (130/0.4) on haemostasis in pregnancy: an in-vitro assessment using thromboelastometry. Eur $\mathrm{J}$ Anaesthesiol. 2010;27: 304-5.

78 Butwick A, Ting V, Ralls LA, Harter S, Riley E: The association between thromboelastographic parameters and total estimated blood loss in patients undergoing elective cesarean delivery. Anesth Analg. 2011;112: 1041-7.

79 Huissoud C, Carrabin N, Audibert F, Levrat A, Massignon D, Berland M, Rudigoz RC: Bedside assessment of fibrinogen level in postpartum haemorrhage by thrombelastometry. BJOG. 2009;116: 1097-102.

80 Dr. A.S. Doucloy-Bouters, Expert meeting, Munich, April 2010

81 Bauters A D-BA, Lejeune C, Provost N, Nobecourt C, Tournoys A, Jude B. : Rotem thromboelastometry in obstetric: near patient-test as an early predictor of post-partum hemorrhage (PPH). J Thromb Haemost. 2007;5 P-S-220

82 Swallow RA, Agarwala RA, Dawkins KD, Curzen NP: Thromboelastography: potential bedside tool to assess the effects of antiplatelet therapy? Platelets. 2006;17: 385-92.

83 Larsen $\mathrm{OH}$, Fenger-Eriksen C, Christiansen K, Ingerslev J, Sorensen B: Diagnostic performance and therapeutic consequence of thromboelastometry activated by kaolin versus a panel of specific reagents. Anesthesiology. 2011;115: 294-302.

84 Scharbert G, Auer A, Kozek-Langenecker S: Evaluation of the Platelet Mapping Assay on rotational thromboelastometry ROTEM. Platelets. 2009;20: 125-30.

85 Lang T, Johanning K, Metzler H, Piepenbrock S, Solomon C, Rahe-Meyer N, Tanaka KA: The effects of fibrinogen levels on thromboelastometric variables in the presence of thrombocytopenia. Anesth Analg. 2009;108: 751-8.

86 Johansson PI, Stissing T, Bochsen L, Ostrowski SR: Thrombelastography and tromboelastometry in assessing coagulopathy in trauma. Scand J Trauma Resusc Emerg Med. 2009;17: 45.

87 Douning LK, Ramsay MA, Swygert TH, Hicks KN, Hein HA, Gunning TC, Suit CT: Temperature corrected thrombelastography in hypothermic patients. Anesth Analg. 1995;81: 608-11. 


\section{Chapter 5b}

\section{Platelet monitoring follow-up in a pregnant patient with HELLP syndrome}

\footnotetext{
Gerhardus J.A.J.M. Kuiper, Marcus D. Lancé, Valerie M. Smit-Fun, Louis L.H. Peeters, Marco A.E. Marcus
}

Based on: Platelets. 2011;22(2):160-3. 


\begin{abstract}
Adequate monitoring of the changes in platelet function in HELLP (haemolysis, elevated liver enzymes, low platelet count) syndrome is important for clinical decision-making. We present a case of a primigravid woman developing HELLP syndrome at 29 weeks and 6 days. Platelet function was assessed by multiple electrode aggregometry and platelet function analyzer (PFA-100). Furthermore, platelet count and mean platelet volume (MPV) were monitored over an eleven days period. The results suggested that the outcome parameters of MPV and PFA-100 are better predictors for platelet function than platelet count.
\end{abstract}




\section{Introduction}

Around $5 \%$ of all pregnancies are complicated by the HELLP syndrome (haemolysis, elevated liver-enzymes and low platelets), in which platelet counts are well below 100 $\times 10^{9} / \mathrm{L}$ [1-3]. Data on platelet function is crucial for timing and planning of delivery, anticipating haemorrhage and choice of anaesthesia technique for the obstetrician and the anaesthesiologist, respectively. Previously, we investigated platelet function in preeclampsia and HELLP syndrome using the Multiplate (MEA; Verum Diagnostica, Munich, Germany) and PFA-100 devices (Siemens Medical Solutions, Europe) [4]. In the present report, in one patient with HELLP syndrome, we performed several analyses at various moments during her pregnancy. It is worthwhile to perform this follow-up on platelet function, because little is known about this regained function over time.

\section{Case report}

We report a 32 year old primigravid woman with history of breast augmentation, lactose intolerance, hay fever and menstrual-cycle dependent migraine. She did not have a history of smoking or drugs- or alcohol (ab)use. The patient did not take any medication. At 29 weeks and 6 days of gestation, she was hospitalized because of the diagnosis HELLP syndrome. She showed arterial hypertension (RR 170/110 $\mathrm{mmHg}$ ), peripheral oedema, epigastric pain and migraine. Blood samples revealed mild liver dysfunction (aspartate amino transferase $177 \mathrm{IU} / \mathrm{L}$, alanine amino transferase 109 and lactate dehydrogenase $843 \mathrm{IU} / \mathrm{L}$ ). The patient was treated for hypertension with methyldopa, labetalol and nifedipine. Magnesium sulphate was added to prevent seizures, and two doses of bethametasone (12 mg intramuscularly) were administered for lung maturity of the child on day of admission (day 0 ) and day 1 , each. Platelet count and function were measured at the same time. In total, we collected 7 blood samples in 11 days (Figures 1 and 2). On the first morning (30 weeks gestation), platelet count was low as was mean platelet volume (MPV). Using the PFA-100, closure times for ADP and epinephrine were below reference values, suggesting platelet dysfunction. Starting from day two, closure times (PFA-100) became normal until delivery. In the first measurement, platelet count was low, whereas MPV was within the normal range. At the third measurement, MPV rose to above normal, while platelet levels were fallen again. At the next measurement, platelet levels had normalized, while the elevated MPV persisted. 
Because of the previously abnormal closure times, we decided to measure platelet function by multiple electrode aggregometry (Multiplate), a technique described in detail by Tóth et al. [5]. As we had no clue of the effect of the standard ADP concentration $(6.4 \mu \mathrm{M})$ in the HELLP syndrome, we used a tailored set of reagents for ADP: $0.5 \mu \mathrm{M}, 2.5 \mu \mathrm{M}$ and $7.5 \mu \mathrm{M}$. However, for collagen and TRAP (thrombin receptor-activating peptide), we used the standard doses (Table 1). Nearly all platelet aggregation measurement using the Multiplate showed results below normal ranges. With collagen stimulation, we recorded low results but within the normal range.

On day 18 (at 32 weeks and 3 days after conception), a healthy girl (birth weight: $2295 \mathrm{~g}$; APGAR: 8/9; umbilical arterial chord $\mathrm{pH}$ 7.27) was delivered by caesarean section under general anaesthesia, because of frank breech presentation. Platelet level at that time was $56 \times 10^{9} / \mathrm{L}$, and the activated partial thromboplastin time (aPTT) was 36 seconds. During operation, the patient received one unit of platelet concentrate because of a risk of bleeding. On day five postpartum, the patient had recovered completely, and she was discharged together with her infant from the hospital. She did not have haemorrhage and her blood pressure was regulated well $(140 / 83 \mathrm{mmHg})$ using labetalol twice daily $200 \mathrm{mg}$. The child received a seven days regimen of antibiotics because of high C-reactive protein (maximum of $32 \mathrm{mg} / \mathrm{dL})$.

Table 1: Platelet aggregation results using Multiplate for patient with HELLP syndrome.

\begin{tabular}{|c|c|c|c|c|c|}
\hline & \multicolumn{3}{|c|}{ ADP (AUC) } & \multirow{2}{*}{$\begin{array}{l}\text { Collagen } \\
3.2 \mu \mathrm{g} / \mathrm{mL}\end{array}$} & \multirow{2}{*}{$\begin{array}{l}\text { TRAP } \\
32 \mu \mathrm{M}\end{array}$} \\
\hline & $0.5 \mu \mathrm{M}$ & $2.5 \mu \mathrm{M}$ & $7.5 \mu \mathrm{M}$ & & \\
\hline Mean & 14.00 & 18.00 & 27.33 & 68.17 & 39.60 \\
\hline Minimum & 4 & 2 & 3 & 21 & 18 \\
\hline Maximum & 25 & 26 & 42 & 101 & 62 \\
\hline Normal Range & n.d. & n.d. & n.d. & $45.9-116.6$ & $94.1-156.3$ \\
\hline
\end{tabular}

Whole-blood platelet aggregation using the Multiplate, starting at the day after admission to the hospital. Platelets were activated with ADP, collagen or TRAP, as indicated. AUC = area under the curve, n.d. not defined. Data are presented as median and inter-quartile ranges. 


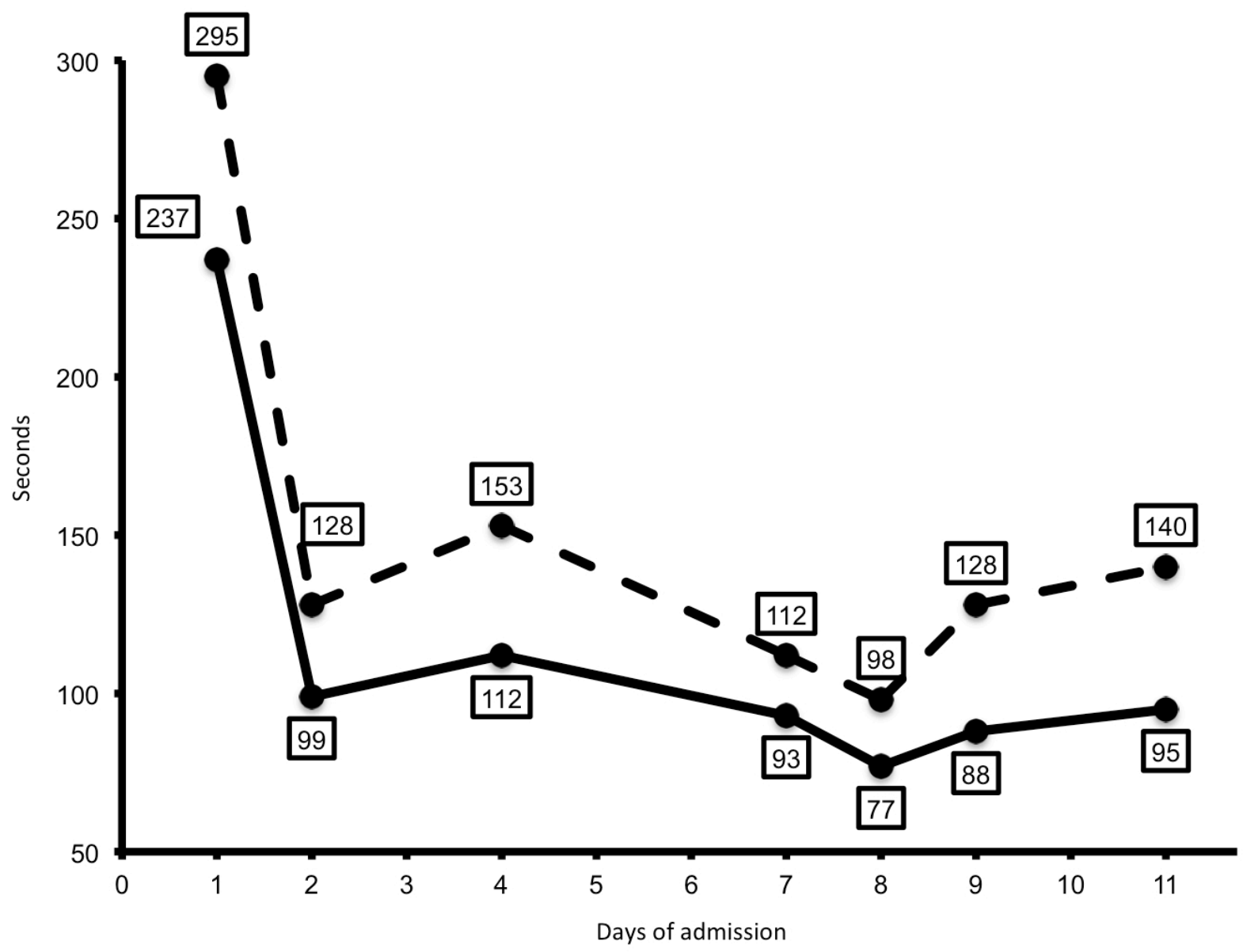

Figure 1: Closure times of patient blood samples using PFA-100 device. Normal range for ADP/collagen (lower curve) cartridges was 55-119 seconds and for epinephrine/collagen cartridges (upper curve) it was 67-171 seconds.

\section{Discussion}

Interestingly, platelet function was out of range on day 1 , and had recovered completely one day later. In an earlier study, we were unable to detect relevant platelet dysfunction using various methods in this specific population [4]. However, we suggested a raised MPV to be a better predictor for regarding risk of bleeding than platelet level itself. Our interpretation of this case is as follows. In the second measurement, platelet size and count were increased, while also closure times with 


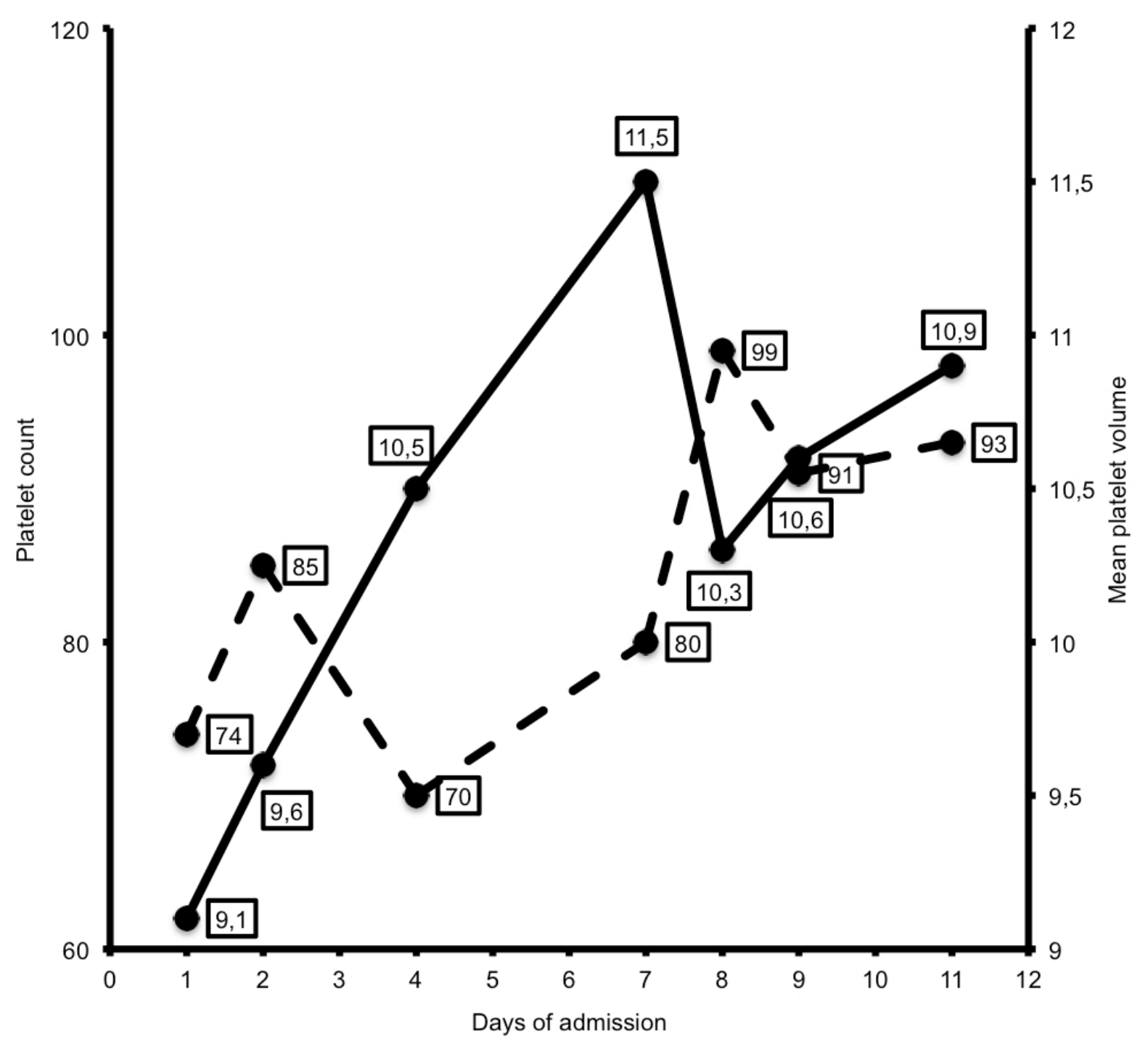

Figure 2: Mean volume (black line) of patient platelets and platelet count (interrupted line). Normal range for mean platelet volume was 8.6-9.7 fL and for platelet count, this was $130-350 \times 10^{9} / \mathrm{L}$.

PFA-100 had normalized. This is consistent with a restoring function of the platelets [6]. Yet, platelet aggregation in all Multiplate measurements was low to normal. This still points to an impaired function. Tóth et al. have shown using ADP as a reagent that the area under the curve $(A \cup C)$ reacts lineary with the dose of ADP [5]. Hence, the maximal $A U C$ of 42 at $7.5 \mu \mathrm{M} A D P$ is still below the normal range, for the standard ADP concentration of 6.4 $\mu \mathrm{M}$ (53.4-122.0 AUC), which again points to an impaired platelet function.

It is clearly conceivable that these low values can be the consequence of the low platelet count, because the platelet aggregation results decrease at counts below $100 \times 10^{9} / \mathrm{L}$ [7]. However, the sudden fall in platelet count to $70 \times 10^{9} / \mathrm{L}$ was not accompanied by changes in PFA-100 results nor by a rise in MPV. This indicated a 
lack of correlation between platelet count and platelet activity. Contrariwise, the later changes in both MPV and PFA-100 results seem to reflect recovery of platelet function. This is in accordance with the idea that MPV can act as a marker for platelet function and consumption [8]. On the other hand, one can speculate that the improvement of MPV is based on an effect of betamethasone treatment, as recently shown by Cerekja et al. [9].

In conclusion, this is the first report on platelet function during the course of HELLP syndrome and recovery. Both MPV and PFA-100 appear to detect the improved hemostasis, and thus can help in monitoring and decision making during the course of HELLP syndrome. However, the variation in platelet function during complicated pregnancies still remains only partly understood. A thorough study is needed to investigate the ideal timing and planning of delivery in pregnancies complicated by HELLP syndrome.

\section{References}

1 Padden MO: HELLP syndrome: recognition and perinatal management. Am Fam Physician. 1999;60: 829-36, 39.

2 Sibai BM, Ramadan MK, Usta I, Salama M, Mercer BM, Friedman SA: Maternal morbidity and mortality in 442 pregnancies with hemolysis, elevated liver enzymes, and low platelets (HELLP syndrome). Am J Obstet Gynecol. 1993;169: 1000-6.

3 Haram K, Svendsen E, Abildgaard U: The HELLP syndrome: clinical issues and management. A Review. BMC Pregnancy Childbirth. 2009;9: 8.

4 Lancé MD, Fun-Smit VM, Timmer, VC, Kroese, A, Marcus, MAE: Impact of platelet function analysis on decision making in preeclampsia and HELLP syndrome. Eur J Anaesth. 2009;26: AP 3-10.

5 Toth O, Calatzis A, Penz S, Losonczy H, Siess W: Multiple electrode aggregometry: a new device to measure platelet aggregation in whole blood. Thromb Haemost. 2006;96: 781-8.

6 Davies JR, Fernando R, Hallworth SP: Hemostatic function in healthy pregnant and preeclamptic women: an assessment using the platelet function analyzer (PFA-100) and thromboelastograph. Anesth Analg. 2007;104: 416-20.

7 Mengistu AM, Mayer J, Boldt J, Rohm KD: Whole-blood aggregometry: are there any limits with regard to platelet counts? Acta Anaesthesiol Scand. 2009;53: 72-6.

$8 \quad$ Threatte GA: Usefulness of the mean platelet volume. Clin Lab Med. 1993;13: 937-50.

9 Cerekja A, Piazze J, Gioia S, Anceschi MM, Cecinato F, Palma E, Pizzulo S, Monaco V, Cosmi EV: Effects on maternal platelet variables of betamethasone administered to achieve fetal lung maturity. Platelets. 2008;19: 78-9. 


\section{Chapter 6a}

Impaired thrombin generation and fibrin clot formation in patients with dilutional coagulopathy during major surgery

Saskia E.M. Schols, Marcus D. Lancé, Marion A. H. Feijge, Jan Damoiseaux, Marco A. Marcus, Karly Hamulyák, Hugo ten Cate, Johan W. M. Heemskerk, Elisabeth C. M. van Pampus

Published in

Thromb Haemost. 2010 Feb;103(2):318-28. 


\section{Abstract}

Background: Patients subjected to hemodilution during surgery are at increased risk of bleeding. We hypothesized that, in the acquired dilutional coagulopathy, insufficient hemostasis is due to either insufficient thrombin generation or insufficient fibrin clot formation.

Methods: In tissue factor-activated plasmas from patients with coagulation deficiency, we measured time curves of thrombin generation and fibrin clot formation (thromboelastography). Investigated were in study A: 10 patients treated with vitamin $\mathrm{K}$ antagonist and 5 healthy subjects; in study $\mathrm{B}$ : 30 patients undergoing cardiopulmonary bypass (CPB) surgery and infused with on average $<2000 \mathrm{~mL}$ crystalloids and colloids (no major bleeding); in study C: 58 patients undergoing major general surgery, and transfused with $>5000 \mathrm{~mL}$ crystalloids, colloids and red cell concentrates, who experienced major bleeding and were post-transfused with fresh frozen plasma.

Results: Treatment with vitamin $\mathrm{K}$ antagonist led to a progressive reduction in thrombin generation but not fibrin clot formation. In CPB patients, plasma factor levels post-surgery were $53-60 \%$ of normal, which was accompanied by moderate reduction in both hemostatic processes. In plasmas from patients undergoing major surgery, factor levels were $38-41 \%$ of normal, and these levels increased after plasma transfusion. Taking preset thresholds for normal thrombin generation and fibrin clot formation, at least one process was low in $88-93 \%$ of the patients with (persistent) bleeding, but only in $40-53 \%$ of the patients without bleeding.

Conclusions: The processes of thrombin generation and fibrin clot formation are independently reduced in acquired dilutional coagulopathy, while minimal levels of both seem to be required for adequate hemostasis. 


\section{Introduction}

Patients undergoing major surgery or trauma are routinely treated with fluids lacking coagulation factors, which leads to an increased risk of bleeding problems due to dilutional coagulopathy. In clinical practice, the indications for impaired hemostasis are currently obtained from conventional coagulation tests, such as the prothrombin time (PT) and activated partial thromboplastin time (aPTT). However, since these single point assays are rather insensitive to coagulation insufficiencies, there is currently is increased interest in the use of integrative tests for the prediction of bleeding risks. Popular tests which provide integrated, time-dependent information on the total activity of the coagulation process include time-dependent measurements of thrombin generation [1, 2] and clot strength using the method of thromboelastography [3-5]. It is supposed that these integrative measurements are valuable for not only the monitoring of coagulopathy but also the management of transfusion [6]. However, why integrative measurements would perform better is unclear, given the still limited knowledge of the altered coagulation process under clinically relevant conditions of in vivo dilution, and in particular because of the unclear relation between the processes of thrombin generation and fibrin clot formation.

Recent data suggest that limited thrombin generation is one of the determinants of the risk of peri-operative bleeding [7]. However, there is a large body of evidence that also the plasma fibrinogen level, which does not play a key role in thrombin generation [8], needs to reach threshold levels to prevent bleeding [9-11]. In the present paper we hypothesized that insufficient hemostasis in dilutional coagulopathy can be due to deficiencies in either thrombin generation or fibrin clot formation. To test this, we performed a first proof-of-principle study with plasmas from patients taking vitamin $\mathrm{K}$ antagonist and from patients experiencing peri-operative dilution with or without bleeding.

\section{Materials and methods}

\section{Materials}

Apyrase and bovine serum albumin were from Sigma (St. Louis, MO, USA). Tissue factor (Innovin) was from Dade Behring (Marburg, Germany). Thrombin substrate ZGly-Gly-Arg aminomethyl coumarin (Z-GGR-AMC) came from Bachem (Bubendorf, Switzerland); human thrombin calibrator and thrombogram software were from Thrombinoscope (Maastricht, The Netherlands). Procoagulant phospholipid vesicles 
(phosphatidyl serine : phosphatidyl choline : phosphatidyl ethanolamine, 1:3:1, mol:mol) were prepared, as described[12]. Thromboelastogram control was from Haemoscope, IPO Medical (Barendrecht, The Netherlands). Other reagents were obtained from Sigma.

\section{Clotting time and factor measurements in plasma}

Measurement of PT was with innovin reagent, of the aPTT with the actin-FSL kit (Dade Behring). Plasma fibrinogen was determined as described [13]. The Behring coagulation system (Dade Behring) was used to measure levels of prothrombin, factor X and factor XIII via a one-stage clotting assay with factor-deficient plasmas. Antithrombin was measured using the same system and a chromogenic assay. Heparin in plasma was determined as anti-factor Xa activity using the Coamatic heparin test (Chromogenics, Mölndal, Sweden). Plasma levels of IgG were quantified using an Immage nephelometer and goat anti-human IgG (Beckman Coulter, Mijdrecht, The Netherlands).

\section{Patients}

The studies were approved by the local Medical Ethics Committee. Blood was taken from patients before and after surgery for clinical determination of standard clotting tests and factor levels. Patients and healthy volunteers did not use other antithrombotic medication than indicated. None of the patients had multiple organ failure, sepsis, or renal or liver dysfunction. Remnant blood samples were used for measurements of non-standard factor levels, thrombin generation and fibrin clot formation. Healthy volunteers and patients gave full informed consent.

Study A. For evaluating the effects of anticoagulant treatment without plasma dilution, blood was obtained from 5 healthy volunteers and from 10 patients, who were using a vitamin $\mathrm{K}$ antagonist (acenocoumarol, $3.13 \pm 1.8 \mathrm{mg} /$ day during $>7$ days; mean $\pm \mathrm{SD}$ ) and were under coagulant control of the Maastricht Anticoagulation Clinic. Based on the international normalized ratio (INR) of plasma PT, the patients were divided into two groups, i.e. one with moderate anticoagulation (INR 1.8-2.6) and one with high anticoagulation (INR 3.6-4.8). Haemostatic parameters of these healthy controls and patients are given in Table 1.

Study $B$. Effects of non-severe dilution were investigated using remnant blood 
Table 1: Haemostatic parameters of healthy subjects and of patients treated with vitamin $\mathrm{K}$ antagonist and moderate or high anticoagulation (study $\mathrm{A}$ ).

\begin{tabular}{lllll}
\hline Parameter & \multicolumn{3}{c}{ Vitamin K antagonism } & \multirow{2}{*}{$\begin{array}{c}\text { Normal } \\
\text { range }\end{array}$} \\
\cline { 2 - 4 } & none $(n=5)$ & moderate $(n=5)$ & high $(n=5)$ & $0.8-1.2$ \\
INR & $1.0 \pm 0.1$ & $2.2 \pm 0.3^{* *}$ & $4.2 \pm 0.5^{* *+}$ & $23-32$ \\
aPTT (s) & $27 \pm 2.1$ & $35 \pm 5.4^{*}$ & $48 \pm 5.7^{* *+}$ & $10-13$ \\
PT (s) & $12 \pm 2$ & $26 \pm 3.8^{* *}$ & $48 \pm 6^{* *}$ & 100 \\
Prothrombin (\%) & $128 \pm 15$ & $43 \pm 18^{* *}$ & $19 \pm 5^{* *}$ & 100 \\
Factor X (\%) & $134 \pm 10$ & $30 \pm 21^{* *}$ & $10 \pm 2^{* *}$ & 100 \\
Antithrombin (\%) & $111 \pm 5$ & $102 \pm 10$ & $108 \pm 10$ & $1.7-4.0$ \\
Fibrinogen (g/L) & $2.8 \pm 0.6$ & $4.1 \pm 1.0$ & $4.4 \pm 0.5^{*}$ & \\
\hline
\end{tabular}

Data are means \pm SD. ${ }^{*} p<0.05$ and ${ }^{* *} p<0.001$ compared to no vitamin $\mathrm{K},{ }^{\dagger} p<0.01$ compared to moderate vitamin $\mathrm{K}$ antagonism.

from 30 consecutive patients undergoing elective cardiopulmonary bypass (CPB)surgery, who did not experience significant blood loss and did not need plasma transfusion. A first blood sample was taken at 30 minutes before start of the surgical procedure, and a second sample was taken at 30 minutes after surgery and administration of protamin. Twenty-four hours before surgery, patients had stopped

Table 2: Characteristics of patients undergoing dilution during surgery of studies $B$ and $C$.

\begin{tabular}{|c|c|c|}
\hline Patient characteristic & Study B & Study C \\
\hline Number of patients $(n)$ & 30 & 58 \\
\hline Male/female $(n)$ & $27 / 3$ & $29 / 29$ \\
\hline Age (years) & $64 \pm 11$ & $62 \pm 13$ \\
\hline Cardiovascular surgery $(n)$ & $30^{1}$ & 21 \\
\hline Abdominal surgery $(n)$ & 0 & 28 \\
\hline Spinal back bone surgery $(n)$ & 0 & 9 \\
\hline Fluid volume infused during surgery $(\mathrm{ml})$ & $1970 \pm 425$ & $>5000$ \\
\hline Fluid type infused & crystalloids, colloids & $\begin{array}{l}\text { crystalloids, colloids, } \\
\text { red cell concentrates }\end{array}$ \\
\hline FFP transfused $(\mathrm{mL})$ & 0 & $810 \pm 270$ \\
\hline Medication during surgery (heparin, IU) & $242 \pm 53$ & 0 \\
\hline Blood sample collected & \multicolumn{2}{|c|}{$1^{\text {st }}: 30$ min before CPB $1^{\text {st }}: 30$ min before FFP } \\
\hline & \multicolumn{2}{|c|}{$2^{\text {nd }}: 30$ min after $\mathrm{CPB}^{2} 2^{\text {nd }}: 30$ min after FFP } \\
\hline
\end{tabular}

Cardiopulmonary bypass (CPB); ${ }^{2}$ blood taken after administration of $245 \pm 80 \mathrm{mg}$ protamin (plasma checked for absence of heparin traces). Data are means \pm SD. 
intake of aspirin (83 $\pm 30 \mathrm{mg} / \mathrm{day})$. During the on-pump stage of the intervention, patients received heparin (242 $\pm 53 \mathrm{IU}$ ), which was antagonized after surgery by administering protamin $(245 \pm 80 \mathrm{mg})$. During the intervention, the patients were infused with $1970 \pm 425 \mathrm{~mL}$ crystalloids (saline) and colloids (venofundin). Patient characteristics are given in Table 2.

Study C. Effects of severe dilution were determined in remnant blood from 58 consecutive patients undergoing major surgery of various forms, e.g. vascular, abdominal or spinal back bone surgery (Table 2). Patients were included who suffered from massive blood loss during or after surgery and therefore needed transfusion of fresh frozen plasma (FFP). These patients had massively been transfused with crystalloids, colloids and a minimum of 10 red cell concentrates (total fluid volume $>5000 \mathrm{~mL}$, corresponding to replacement of one blood volume). To stop bleeding, the patients had received on average 2.7 bags of FFP $(870 \pm 270 \mathrm{~mL})$. Additional clinical criteria for transfusion of FFP: aPTT $>40 \mathrm{~s}$ (37 patients), PT >16 sec (17 patients), and fibrinogen level $<2.0 \mathrm{~g} / \mathrm{L}$ (41 patients), such in accordance with international guidelines [14] [15]. Blood samples were obtained at approximately 30 minutes before and 30 minutes after FFP transfusion (Table 2). If bleeding persisted, the patients were at a later stage transfused with additional red cell concentrates, FFP and/or platelets, as required. For 4 patients, insufficient remnant plasma was obtained for complete measurement sets of fibrin clot formation.

\section{Plasma preparation}

Remnant blood was used, taken by venous puncture and anticoagulated with sodium citrate (1/10 volume, $12.9 \mathrm{mM}$ f.c.). Platelet-poor-plasma was prepared by centrifuging twice at $2630 \mathrm{~g}$ for 10 minutes at room temperature. Plasma samples were snap-frozen at $-80^{\circ} \mathrm{C}$ until use.

\section{Thrombin generation measurement}

Thrombin generation in patient plasma was continuously measured according to the calibrated automated thrombogram assay, with slight modifications [13, 16]. Citrateanticoagulated plasma (12.9 mM trisodium citrate) was supplemented with phospholipids $(4 \mu \mathrm{M})$. Coagulation was triggered with $16.6 \mathrm{mM} \mathrm{CaCl} 2$ and an optimized concentration of $10 \mathrm{pM}$ tissue factor [17]. Thrombin activity was continuously followed at $37^{\circ} \mathrm{C}$ from the cleavage of thrombin substrate, Z-GGR-AMC 
(2.5 mM). Samples were run in triplicate, and a calibrator was used for each plasma to give calibrated curves of thrombin activity. First-derivative traces were converted to nanomolar concentrations of thrombin, as described [16]. Thrombin peak height and area-under-the-curve were used as most sensitive determining parameters describing the thrombin generation process [17, 18]. Intra-assay variation coefficient was $6 \%$. Normal value of the thrombin peak height was $314 \pm 24 \mathrm{nM}$ (mean $\pm \mathrm{SD}$, $n=40$ healthy subjects). A cut-off level for normal thrombin generation was arbitrarily set at mean $-2.5 \times \mathrm{SD}$, i.e. at $194 \mathrm{nM}$.

\section{Thromboelastography measurement}

Thromboelastography was performed using a TEG 5000 Thrombelastograph hemostasis analyzer (Haemoscope, Niles, IL, USA), measuring the torsion between a slowly rotating plastic cup and a stationary pin [19]. Samples of $340 \mu \mathrm{L}$ citrateanticoagulated plasma were supplemented with phospholipids $(4 \mu \mathrm{M})$, and coagulation was triggered with $10 \mathrm{pM}$ tissue factor and $\mathrm{CaCl}_{2}(16.6 \mathrm{mM})$. Key evaluation parameters of thromboelastograms were the $\alpha$-angle and the maximal amplitude, which inform on the rate of fibrin strand formation and the clot amount/strength, respectively [20]. Note that experiments were carried out in the absence of platelets. Maximal amplitude was taken as measure of total fibrin clot formation, sensitive to plasma dilution [17]. Normal value of the maximal amplitude measured in plasma from 40 healthy volunteers was $42 \pm 5.7 \mathrm{~mm}$ (mean $\pm \mathrm{SD}$ ); the cut-off level for normal fibrin clot formation was arbitrarily set at $13.5 \mathrm{~mm}$ (mean - 2.5 $\times \mathrm{SD})$.

\section{Statistics}

Peri-operative effects of fluid infusion and FFP transfusion were evaluated with the paired sample t-test. Factor contributions to thrombin generation and fibrin clot formation were evaluated by multivariate regression analysis. Correlations between parameters were determined using the $X^{2}$ test. Patient groups were compared with a one-way ANOVA test. The statistical package for the social sciences 15.0 (SPSS, Chicago, IL, USA) was used. 


\section{Results}

Effect of plasma dilution on thrombin generation and fibrin clot formation after treatment with vitamin $\mathrm{K}$ antagonist

To determine how anticoagulant therapy affects thrombin generation and fibrin clot formation without dilution, plasma was used from healthy subjects and patients receiving a vitamin $\mathrm{K}$ antagonist (Table 1 ). In the patient plasmas, levels of vitamin $\mathrm{K}$ dependent coagulation factors were reduced to $30-43 \%$ (INR 2.2) and 10-19\% (INR 4.2) of normal. In both patient groups, plasma antithrombin levels were in the normal range, while fibrinogen levels were relatively high i.e. on average 4.1-4.4 g/L.
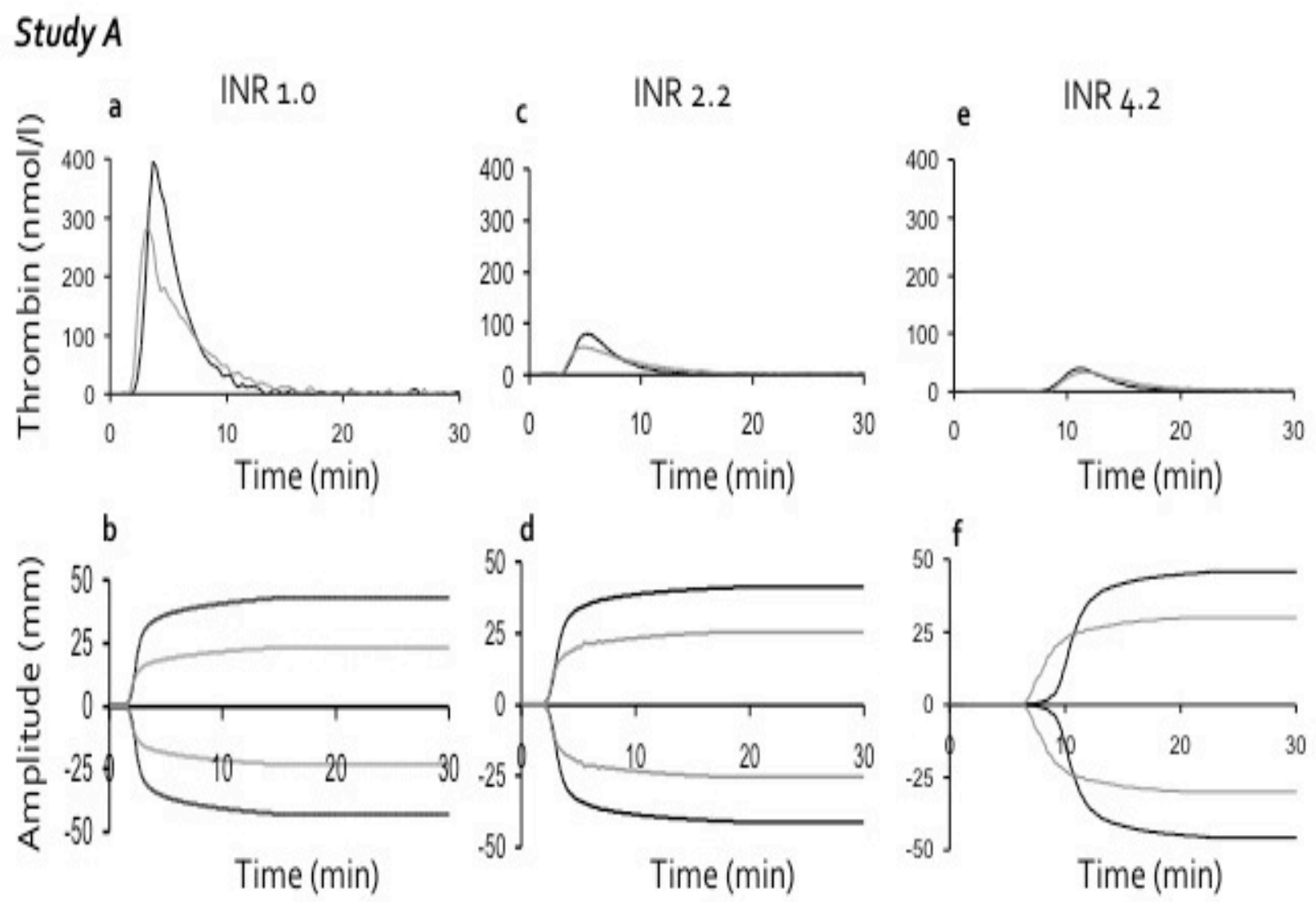

Figure 1: Kinetics of thrombin generation and fibrin clot formation in plasma from healthy subjects and patients treated with vitamin $\mathrm{K}$ antagonist. Study $A$ : plasma was used from 5 healthy volunteers $(\mathrm{a}, \mathrm{b}$; INR 1.0); plasma was also obtained from patients treated with a vitamin $\mathrm{K}$ antagonist: 5 patients with moderate anticoagulation (c, d; INR 2.2) and 5 patients with high anticoagulation (e, f; INR 4.2). Plasma was used either undiluted (black curves) or diluted with $50 \%$ saline containing $12.9 \mathrm{mM}$ citrate (grey curves). Plasma was incubated with phospholipids $(4 \mu \mathrm{M})$, and activated with $10 \mathrm{pM}$ tissue factor and $16.6 \mathrm{mM} \mathrm{CaCl}_{2}$. Shown are for each patient group representative curves of thrombin generation $(a, c, e)$ and fibrin clot formation (b, d, f). 
Coagulation activities were tested in these plasmas, either undiluted or after dilution in vitro with saline, by triggering with an optimal concentration of $10 \mathrm{pM}$ tissue factor in the presence of $4 \mu \mathrm{M}$ procoagulant phospholipids [17]. Curves of thrombin generation indicated that an increase in INR from 1.0 to 2.2 was accompanied by a strong reduction in thrombin peak height, changing from about 400 to $100 \mathrm{nM}$ (Figure 1, upper pane/s). In plasma from patients with high anticoagulation (INR 4.2), thrombin generation was more delayed and reduced giving a peak height of $50 \mathrm{nM}$. Fifty percent dilution of normal plasma with saline moderately reduced the thrombin peak height to $300 \mathrm{nM}$, but this was of little additive effect in patient plasmas (Figure 2a). Regression analysis of thrombin generation parameters with plasma factor levels showed that the thrombin peak height strongly correlated with prothrombin (Figure $2 \mathrm{~b})$ and factor $\mathrm{X}\left(R^{2}=0.97, p<0.001\right)$. This supported the notion that both coagulation factors are key predictive variables of the thrombin-generating process [7, 21, 22]. In contrast, plasma fibrinogen levels did not correlate with thrombin generation parameters $(p>0.20)$.

Thromboelastography was used to measure time-dependent formation of fibrin clots in these plasma samples, applying the same optimal triggering conditions of 10 pM tissue factor and $4 \mathrm{mM}$ procoagulant phospholipids (Figure 1, lower pane/s). Overall, the lag time to start of fibrin clot formation was similar to that of thrombin generation, and corresponded with the PT values. Strikingly, the rate of fibrin formation ( $\alpha$-angle of curve) and the maximal extent of fibrin formation (maximal amplitude) were not diminished in plasmas with INR of 2.2 or 4.2 , in spite of the marked reduction in thrombin generation. On the other hand, fibrin formation did substantially decrease upon $50 \%$ dilution with saline in both control and patient plasmas (Figure 2c). The fibrinogen level was a main predictive variable of maximal amplitude in undiluted or diluted plasmas (Figure 2d), but the levels of prothrombin and factor $X$ failed to correlate with thromboelastography parameters $(p>0.52)$. Taken together, we find that the reduction in coagulation factor levels due to intake of vitamin $\mathrm{K}$ antagonist is accompanied by impaired thrombin generation, but not by a corresponding reduction in fibrin clot formation. 


\section{Study A}

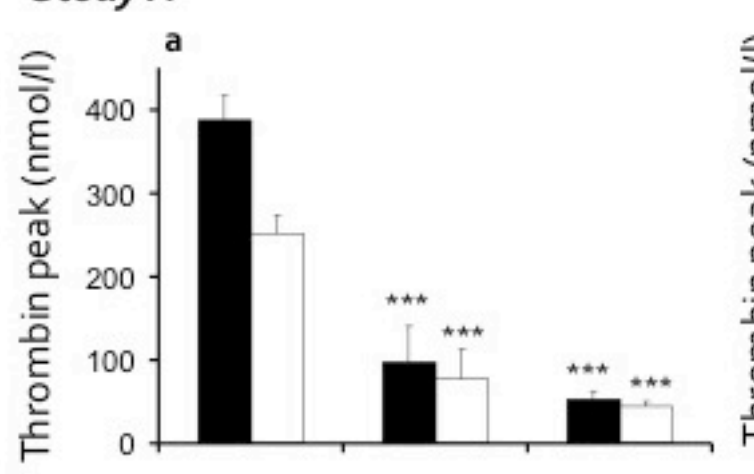

INR $1.0 \quad$ INR $2.2 \quad$ INR 4.2
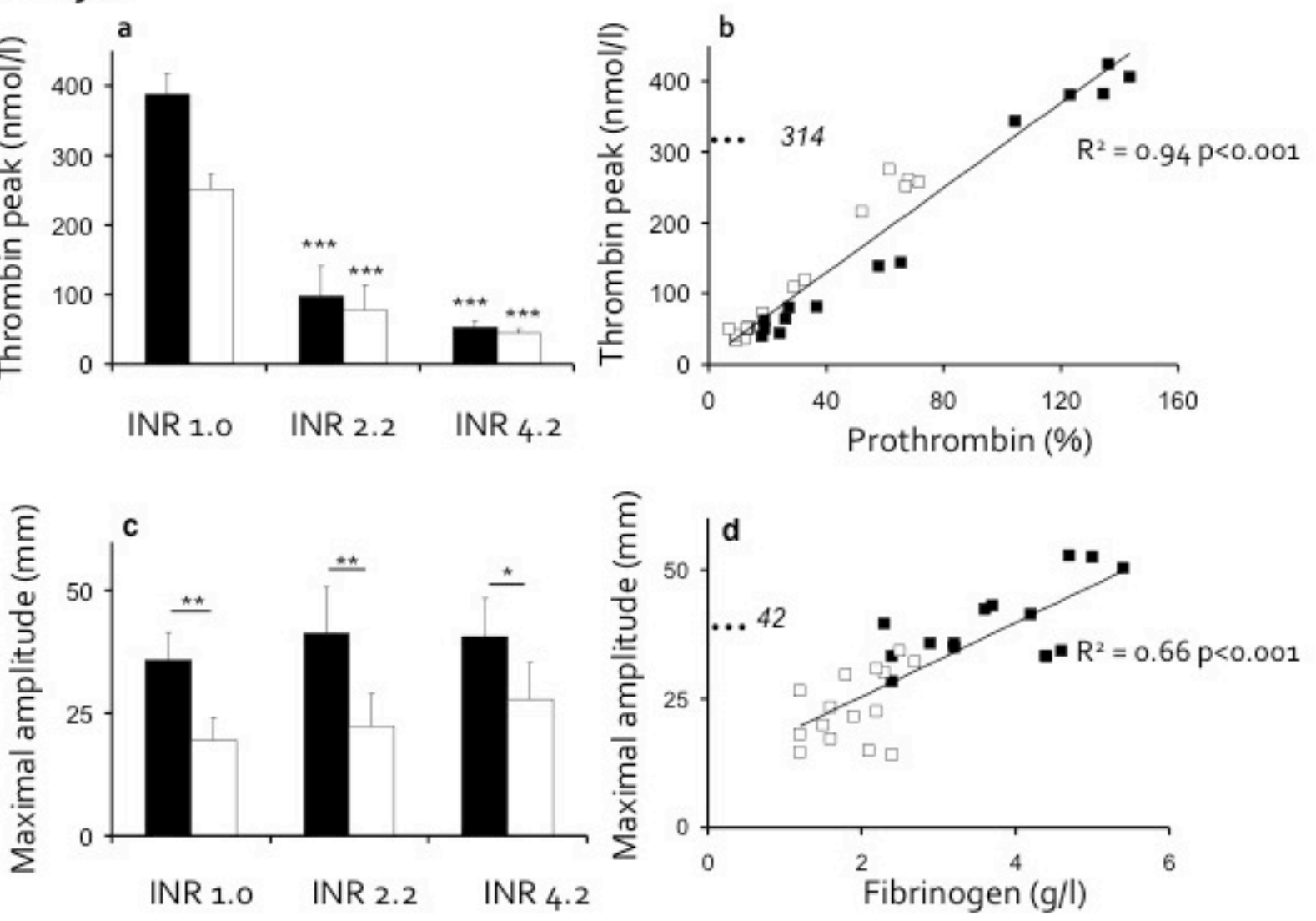

Figure 2: Parameters of thrombin generation and fibrin clot formation in plasma from healthy subjects and patients treated with vitamin $\mathrm{K}$ antagonist. Plasma was used from healthy volunteers (INR 1.0) and patients with moderate (INR 2.2) or high (INR 4.2) anticoagulation (study A). Plasma was undiluted (black bars and symbols) or diluted $50 \%$ with saline (white bars and symbols), as in Figure 1. Thrombin generation was measured upon plasma triggering with tissue factor and $\mathrm{CaCl}_{2}$. Data give the averaged thrombin peak height (a), and correlation per sample with plasma prothrombin level (b). Fibrin clot formation was measured at the same activation conditions. It was quantified as maximal clot amplitude (c); further, correlation per sample with fibrinogen level (d). Dotted lines at $y$-axis represent values of normal pooled plasma (mean $\pm \mathrm{SD}, n=5$ ); ${ }^{*} p<0.05, \quad{ }^{* *} p<0.01,{ }^{* * *} p<0.001$ compared to corresponding control group.

Thrombin generation and fibrin clot formation in plasmas from patients with perioperative dilution during surgery

Two studies were performed with plasmas from surgical patients, who were undergoing in vivo plasma dilution due to fluid infusion. Study B involved 30 patients subjected to CPB surgery (Table 2). None of these patients showed significant bleeding during or after the intervention so that FFP transfusion was not required. In 
study C, plasmas were investigated from 58 patients subjected to major surgery of various forms. Because of the presence of major bleeding during or after surgery, these patients were transfused with FFP. In both studies B and C, the infusion of fluids during surgery resulted in a prolongation of routine clotting times and in a lowering of the plasma levels of (anti)coagulant factors. For study B, levels of prothrombin, factor $\mathrm{X}$, factor XIII, fibrinogen and antithrombin were reduced to 53$60 \%$ of normal. In patient plasmas of study $C$ (before FFP transfusion), these levels were more strongly reduced to $38-41 \%$ of normal (Table 3 ). This was also concluded from the markedly lower level of immunoglobulin lgG, which was taken as an independent measure of plasma dilution. In this study, the transfusion of FFP resulted in a significant improvement of plasma coagulation factors and IgG.

As a consequence of the CPB procedure (study B) - in which plasmas were diluted in vivo -, the curves of thrombin generation and fibrin clot formation were markedly lowered (Figure 3a). In comparison to the normal range of thrombin peak height of $314 \pm 24 \mathrm{nM}$ (mean $\pm \mathrm{SD}, n=40$ ) [7], this level was within or slightly below this range in plasmas from patients prior to surgery (Figure 4a). After CPB surgery,

Table 3: Haemostatic parameters of patients in studies $B$ and $C$.

\begin{tabular}{|c|c|c|c|c|c|}
\hline \multirow[b]{2}{*}{ Parameter } & \multicolumn{2}{|c|}{ Study B $(n=30)$} & \multicolumn{2}{|c|}{ Study C $(n=58)$ Normal } & \multirow[b]{2}{*}{ range } \\
\hline & before CPB & after CPB & before FFP & after FFP & \\
\hline Platelets $\left(\times 10^{9} / \mathrm{L}\right)$ & $235 \pm 82$ & $153 \pm 57$ & $88 \pm 43$ & $89 \pm 32$ & $130-350$ \\
\hline Haematocrit & $0.40 \pm 0.04$ & $0.27 \pm 0.04^{* \star *}$ & $0.26 \pm 0.06$ & $0.26 \pm 0.05$ & $0.36-0.52$ \\
\hline $\mathrm{aPTT}(\mathrm{s})$ & $28 \pm 2.1$ & $33 \pm 2.9^{* \star *}$ & $62 \pm 33$ & $40 \pm 12^{\star \star \star}$ & $23-32$ \\
\hline PT (s) & $11 \pm 0.4$ & $14 \pm 1.2^{* * *}$ & $16 \pm 2.7$ & $13 \pm 1.6^{\star \star \star}$ & $10-13$ \\
\hline Prothrombin (\%) & $94 \pm 16$ & $59 \pm 16^{* * *}$ & $38 \pm 13$ & $49 \pm 14^{* * *}$ & 100 \\
\hline Factor X (\%) & $83 \pm 13$ & $53 \pm 13^{* * *}$ & $40 \pm 15$ & $51 \pm 15^{\star \star *}$ & 100 \\
\hline Factor XIII (\%) & $91 \pm 16$ & $60 \pm 14^{* \star *}$ & $41 \pm 15$ & $52 \pm 15^{\star \star \star}$ & 100 \\
\hline Antithrombin (\%) & $82 \pm 16$ & $53 \pm 13^{* * *}$ & $38 \pm 11$ & $50 \pm 13^{* \star *}$ & 100 \\
\hline Fibrinogen (g/L) & $3.5 \pm 1.0$ & $2.0 \pm 0.5^{* * *}$ & $1.3 \pm 0.7$ & $1.7 \pm 0.8^{* * *}$ & $1.7-4.0$ \\
\hline $\lg G(g / L)$ & $7.6 \pm 1.8$ & $5.2 \pm 2.0^{\star \star \star}$ & $3.8 \pm 1.6$ & $4.6 \pm 1.6^{\star \star \star}$ & 7.0-16 \\
\hline
\end{tabular}

Means $\pm \mathrm{SD} ;{ }^{* *} p<0.01,{ }^{* * *} p<0.001$ compared to first plasma sample. 
the thrombin peak height dropped substantially from $284 \pm 43$ to $212 \pm 67 \mathrm{nM}$ $(p<0.001)$. Arbitrarily, we set a cut-off value for low thrombin generation at a peak height of <194 nM (i.e. the normal mean - $2.5 \times$ SD). Using this threshold, 8 out of 30 patients had a low thrombin generation profile. Similarly, thromboelastogram curves showed a significant reducing effect of the CPB procedure on fibrin clot formation (Figure 3b).Overall, the maximal amplitude decreased from $32 \pm 6.5$ to $17 \pm 8.3 \mathrm{~mm}$ $(p<0.001)$. Again, we set a cut-off value for low fibrin clot formation at a maximal amplitude of $<13.5 \mathrm{~mm}$ (i.e. normal mean $-2.5 \times \mathrm{SD}$ ). In this case, plasmas from 8 patients were low in clot formation after surgery (Figure $4 b$ ).

In study $C$ the effect of FFP transfusion could be investigated. In most patients, this transfusion increased thrombin generation as well as fibrin clot
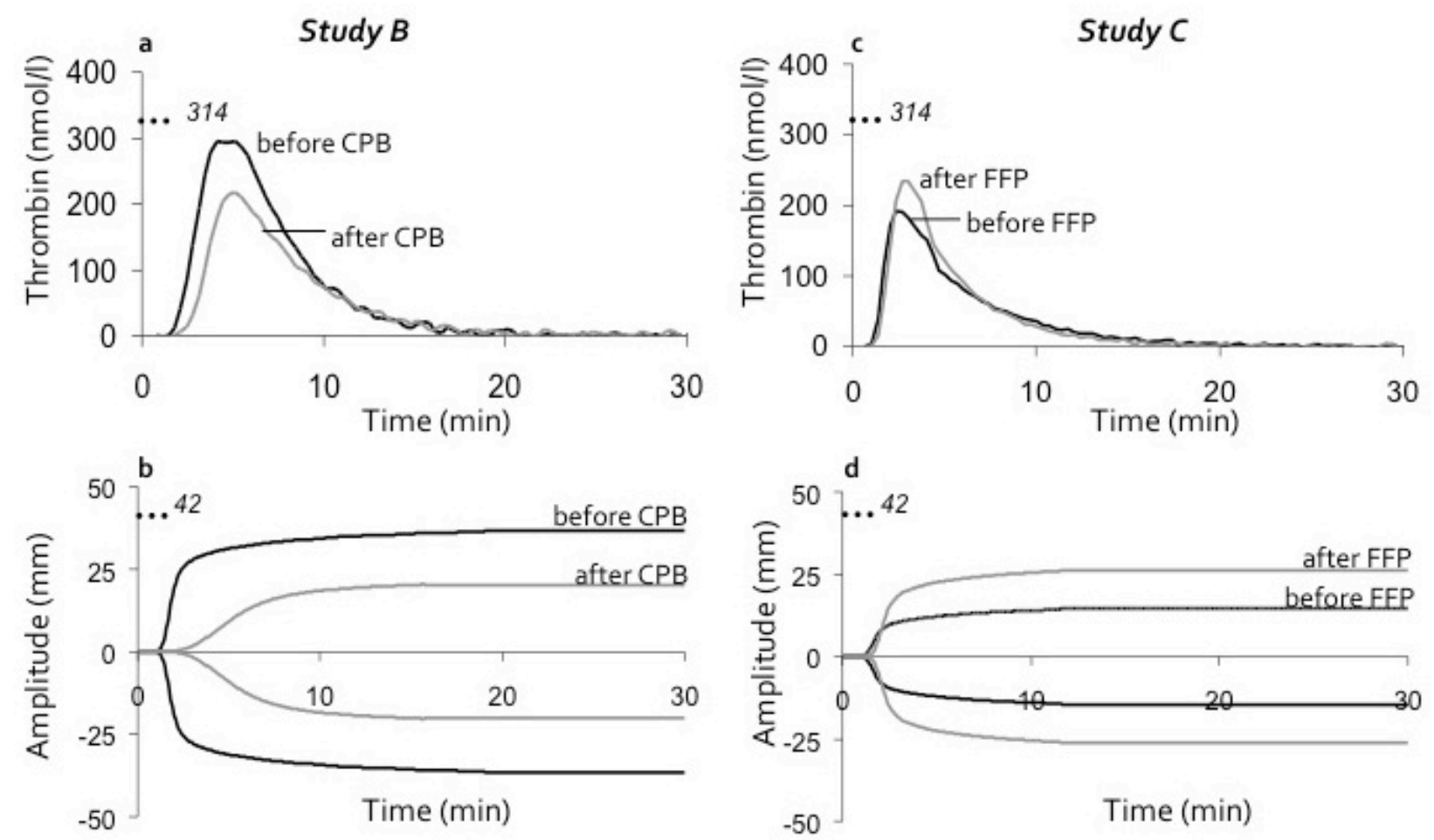

Figure 3: Kinetics of thrombin generation and fibrin clot formation in plasma from patients subjected to dilution during surgery. Study B: plasma was obtained from patients before and after CPB surgery (a, b). Study C: plasma was obtained from patients undergoing major general surgery before and after FFP transfusion (c, d). Plasma was supplemented with phospholipids (4 $\mu \mathrm{mol} / \mathrm{l})$ and used for measurement of thrombin generation and fibrin clot formation, following activation with tissue factor and $\mathrm{CaCl}_{2}$ (see Figure 1). Shown are curves of thrombin generation $(\mathrm{a}, \mathrm{c}$ ) and of fibrin clot formation (b, d) for a representative patient before (black curves) and after (grey curves) intervention or transfusion. Dotted lines at $y$-axis represent values with normal pooled plasma. 
formation, as is illustrated in Figure 3c-d for a patient with stopped bleeding. Prior to transfusion, in the majority of patients the thrombin peak height and the maximal clot amplitude were below the cut-off values. Thus, plasmas from 41/54 patients were low in thrombin generation and plasmas from 41/54 patients had low fibrin clot formation curves (Figure 4c-d). In general, transfusion with FFP significantly increased the
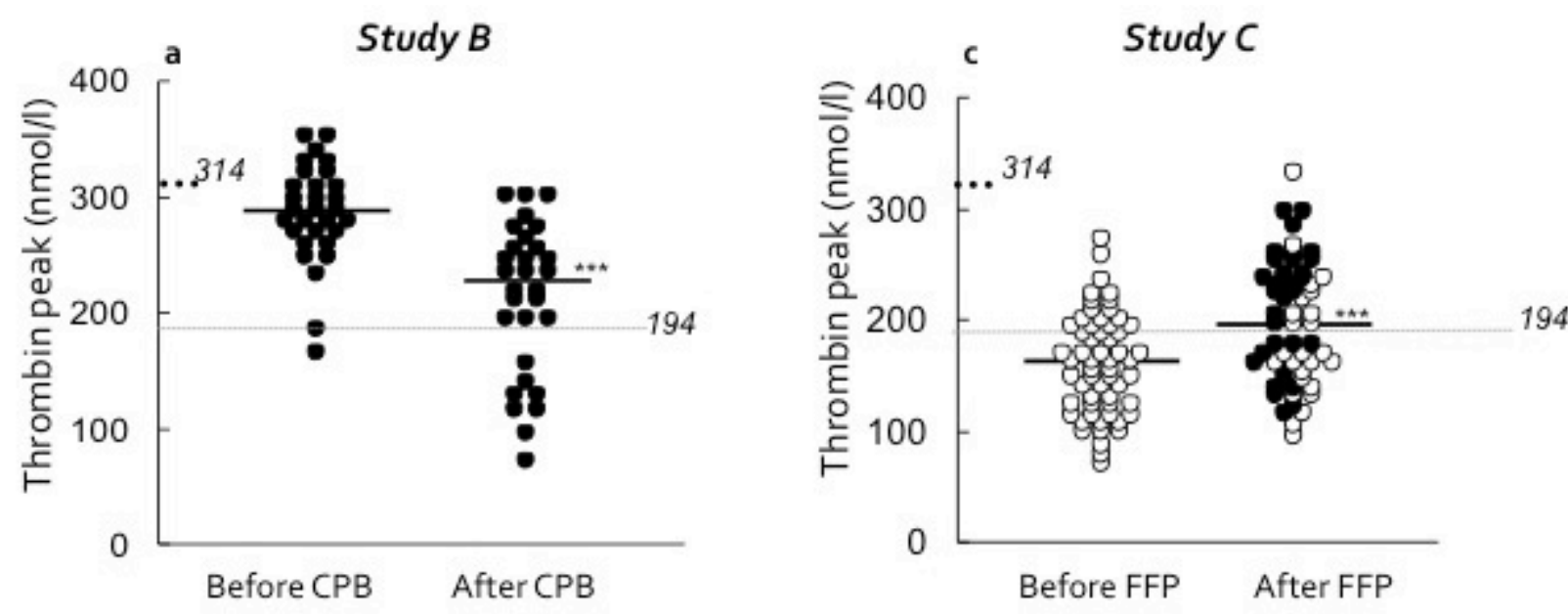

b

d
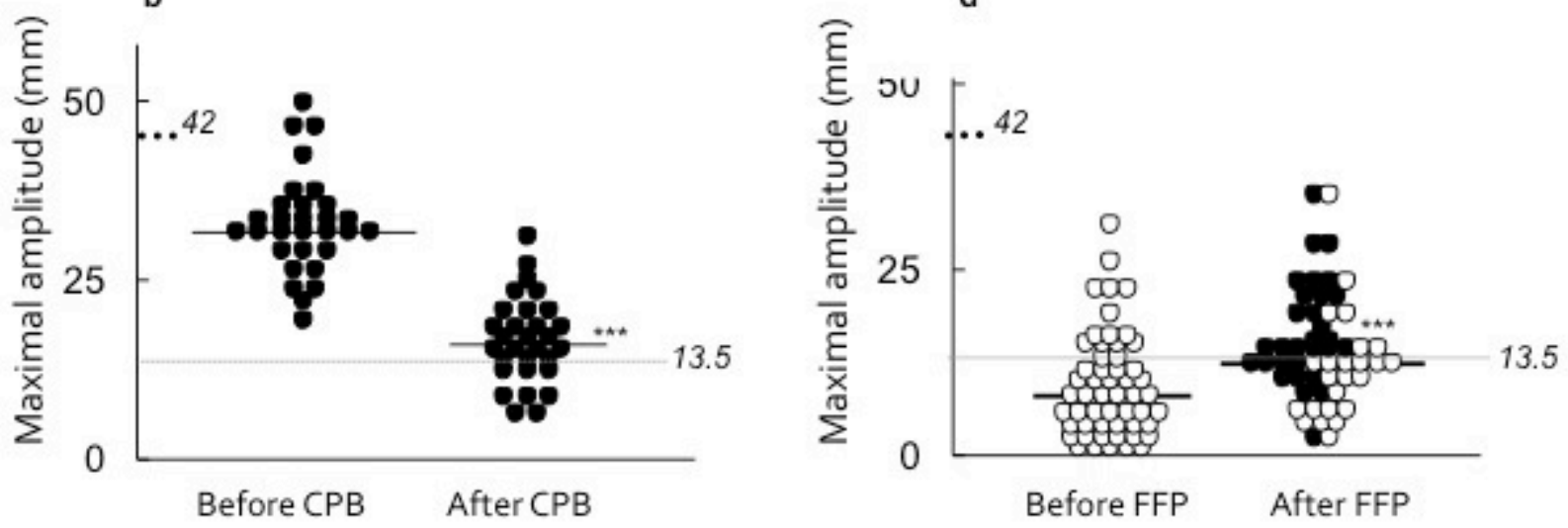

Figure 4: Parameters of thrombin generation and fibrin clot formation in plasma from patients subjected to dilution during surgery. Plasma was used from patients before and after CPB surgery (study B: $a, b$ ); or from patients undergoing major general surgery before and after FFP transfusion (study C: c, d). Measurements of thrombin generation and fibrin clot formation were as in Figure 3. Data are thrombin peak height before/after surgery or transfusion $(a, c)$; further, maximal amplitude of clot formation before/after surgery or transfusion $(b, d)$. White dots represent patients with bleeding before or after FFP transfusion; black dots represent patients without bleeding or with stopped bleeding. Dotted lines at $y$-axis give values with normal pooled plasma. Dotted continued lines give arbitrary cut-off values for normal thrombin or fibrin clot formation (mean $-2.5 \times \mathrm{SD}$ ). ${ }^{* *} p<0.001$ compared to pre-surgery or pre-transfusion. 
thrombin peak height (from $159 \pm 44$ to $196 \pm 53 \mathrm{nM}, p<0.001$ ) and the maximal amplitude of thromboelastography (from $10 \pm 7.4$ to $14 \pm 7.7 \mathrm{~mm}, p<0.001$ ). Yet, even after transfusion, the plasmas from 27 and 28 patients remained below the cut-off levels for normal thrombin generation and fibrin clot formation, respectively. After FFP transfusion, bleeding stopped in 30/58 patients and continued in the other 24
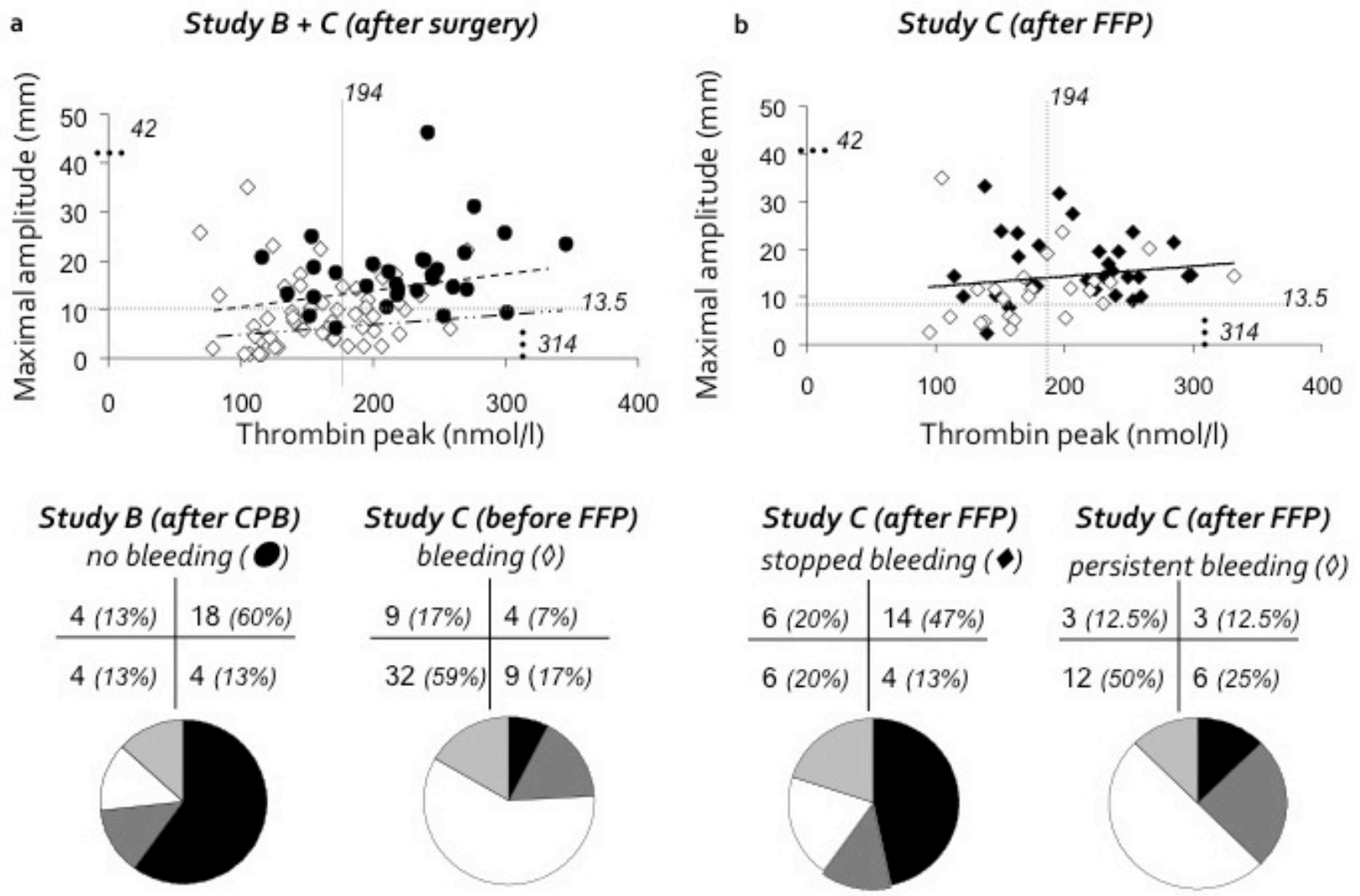

Figure 5: Effect of dilution during surgery on thrombin generation and fibrin clot formation. Plasma was used from patients after CPB surgery (study B) and from patients undergoing major surgery before and after FFP transfusion (study $\mathrm{C}$ ). Measurements of thrombin generation (TG) and fibrin clot formation (FCF) were as in Figure 3. (a) Plasma from patients under diluted condition, i.e. patients after CPB surgery $(\bullet)$ or patients with bleeding after general surgery before FFP transfusion $(\diamond)$. (b) Plasma from patients after FFP transfusion with stopped bleeding $(\diamond)$ or with persistent bleeding $(\diamond)$. Graph plots: Correlation of thrombin peak height and maximal clot formation (maximal amplitude) for individual patients. Normal and cut-off levels of thrombin generation and fibrin clot formation are set as in Figure 4. Cut-off for normal thrombin peak height was at $194 \mathrm{nmol} / /$; cut-off for normal clot formation was at maximal amplitude of $13.5 \mathrm{~mm}$. Quadrants and pie-charts (clock-wise): numbers of patients (percentages) with normal TG and normal FCF $(\bullet)$; normal TG and low FCF $(\varpi)$; low TG and low FCF (口); low TG and normal FCF ( $\square$ ). 
patients. It was relevant to compare the assay parameters in the plasmas from patients with stopped bleeding and the plasmas from patients with continued bleeding after transfusion. Typically, these were significantly higher in the group of patients with stopped bleeding in comparison to the bleeding group: thrombin peak height of $207 \pm 51$ vs. $179 \pm 54 \mathrm{nM}(p<0.05)$, and maximal clot amplitude of $16 \pm 7$ vs. $12 \pm 7 \mathrm{~mm}(p<0.01)$, respectively.

By combining the results of studies $B$ and $C$, analysis was possible of the relations between bleeding, thrombin generation and fibrin clot formation. Therefore, thrombin peak heights and maximal amplitudes of thromboelastography were compared in regression plots (Figure 5a). The patients of study B with no or minor bleeding were assigned as 'non-bleeding', while those of study $\mathrm{C}$ requiring FFP transfusion were indicated as 'bleeding'. For all patients together, the parameters of both assays were related $\left(R^{2}=0.11, p=0.002\right)$, but this was not the case for patients of only study B $\left(R^{2}=0.05, p=0.22\right)$ or only study $C\left(R^{2}=0.010, p=0.47\right)$. When using the preset cut-off values for low thrombin generation and fibrin clot formation, the majority of non-bleeding patients $(60 \%)$ gave assay values which were both in the normal range, while this was the case for $7 \%$ of the bleeding patients. Conversely, plasmas from $13 \%$ of the non-bleeding patients, but $59 \%$ of the bleeding patients were low in both thrombin generation and fibrin clot formation $\left(x^{2}=0.30\right.$, $p<0.001)$. Overall, $26 \%(13+13 \%)$ of the non-bleeding and $34 \%(17+17 \%)$ of the bleeding patients scored low in one of the two assays. Regarding study $\mathrm{C}$, a similar comparison was made for patients with stopped or persistent bleeding after FFP transfusion (Figure $5 \mathrm{~b}$ ). In this case, plasmas from $47 \%$ of the patients with stopped bleeding were normal in thrombin generation and fibrin clot formation, while this was the case for only $12.5 \%$ of the patients with persistent bleeding. Low values of thrombin peak height and maximal amplitude were present in $20 \%$ and $50 \%$ of the patients with stopped and persistent bleeding, respectively $\left(X^{2}=0.10, p<0.05\right)$. Low values for one of the two assays were detected in 33\% $(20+13 \%)$ and $37.5 \%(12.5+$ $25 \%$ ) of patient plasmas. An indication for the relevance of this difference came from the observation that plasma levels of prothrombin, factor $X$, fibrinogen and IgG were significantly higher in the group with stopped bleeding than in the group with persistent bleeding $(p<0.05)$. Based on the pre-set cut-off values, it was concluded that both thrombin generation and fibrin clot formation are low in $50-59 \%$ of the patients with bleeding, but in only $13-20 \%$ of cases without or with stopped bleeding. 
This difference is illustrated in the pie-charts of Figure 5.

Predictive variables of impaired thrombin generation and fibrin clot formation in dilution

Plasma IgG may be a useful marker of in vivo dilution, which is independent of changes in coagulation. The $\lg$ level indeed was higher in plasmas from the nonbleeding patients of study $B(5.2 \pm 2.0 \mathrm{~g} / \mathrm{L})$ than from the bleeding patients of study $C$ $(3.8 \pm 1.6 \mathrm{~g} / \mathrm{L})$ (Table 3). For both studies together, the $\lg \mathrm{G}$ level correlated with the concentrations of fibrinogen, prothrombin and factor X (Figure 6a-c). In addition, IgG correlated with the factor concentrations in post-transfusion plasmas of study $\mathrm{C}$ (Figure 6d-f). The level of $\mathrm{lgG}$ was $<4.5 \mathrm{~g} / \mathrm{L}$ in most plasmas from patients with persistent bleeding, and it was not markedly higher in plasmas from non-bleeding patients (Figure 6, open and closed bullets). Correlation analysis showed significant, albeit weak correlations between IgG level and thrombin peak height $\left(R^{2}=0.27\right.$, $p<0.001)$ or maximal amplitude $\left(R^{2}=0.15, p=0.001\right)$. Together, these results suggested that plasma IgG is a covariant of coagulant activity, rather than an independent parameter of dilution.

Using multiple regression analysis, we analyzed correlations of key variables of both tests for plasmas of studies $B$ and $C$ (Table 4). In thrombin generation curves, the endogenous thrombin potential (area-under-the-curve) correlated strongly with the thrombin peak height in all comparisons. Similarly, in curves of fibrin clot formation, the $\alpha$-angle (rate of fibrin formation) co-varied with the maximal amplitude. Levels of factor $X$ and $\lg G$ significantly contributed to the variation in thrombin generation, while only fibrinogen explained part of the variation in fibrin clot formation. Separate regression analysis of the data from one of the studies did not yield markedly different results. Only in study $\mathrm{C}$, prothrombin (in pre-FFP samples) contributed to the variation in thrombin peak height, while factor XIII contributed to the variation in maximal thromboelastography amplitude. With respect to conventional clotting times, the prolonged aPTT surgery correlated with the thromboelastography assay $\left(R^{2}=0.25, p=0.024\right)$. The aPTT of plasmas from patients with stopped bleeding (study $\mathrm{C}$ ) correlated with thrombin peak height $\left(R^{2}=\right.$ $0.15, p=0.022$ ). For all patients together (studies B and $C$ ), the aPTT correlated with the assays of thrombin generation and fibrin clotin plasma samples of study $B$ after 

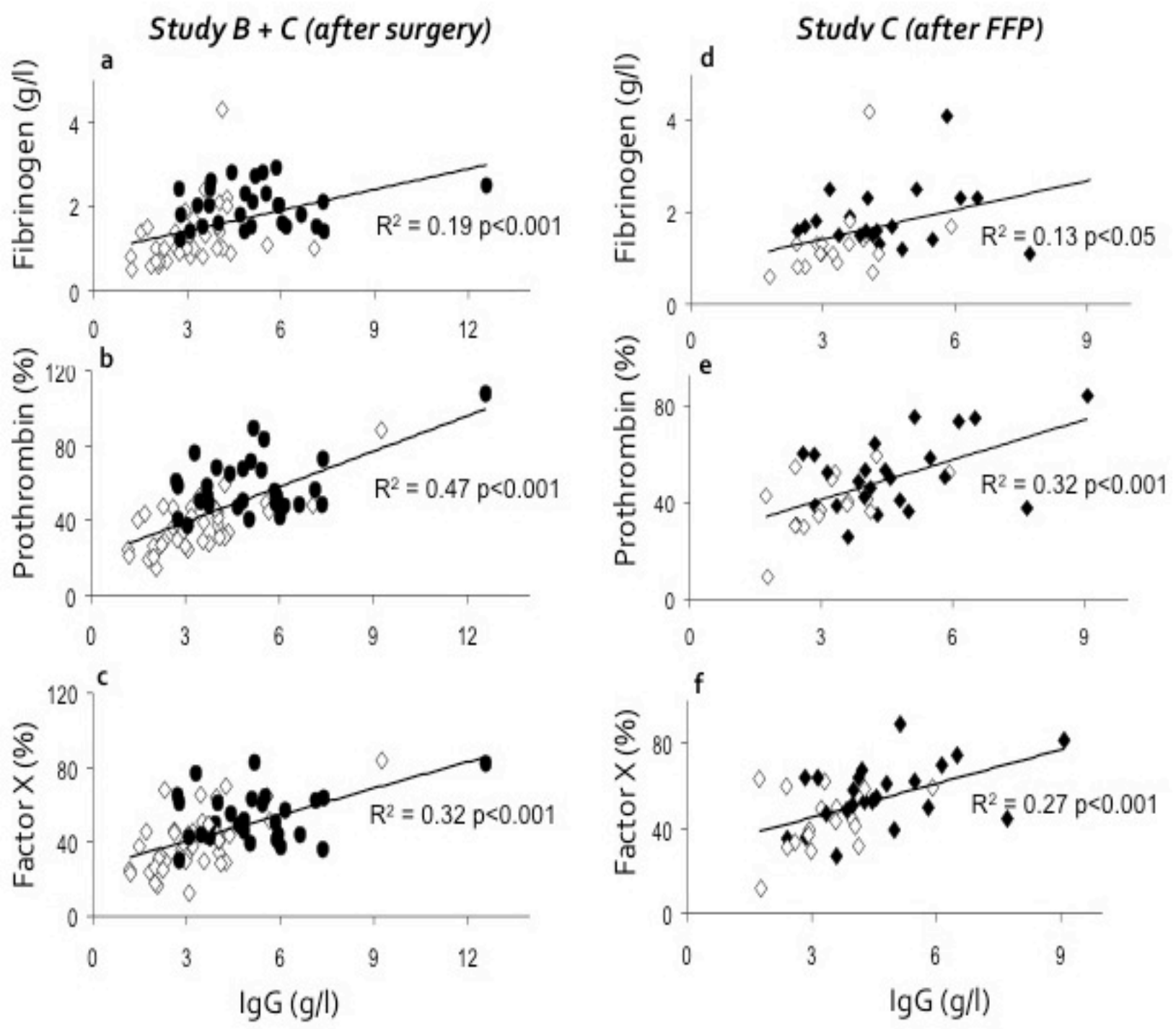

Figure 6: Correlation of plasma IgG and factor levels. ( $a, b, c)$ Plasmas from patients with dilution during surgery. Factor levels were measured from patients after CPB surgery (study $B, \bullet$ ) and patients with bleeding after major surgery before FFP transfusion (study $C, \diamond)$. (d, e, f) Plasma from patients after FFP transfusion (study C). Dots represent patients with stopped bleeding $(\diamond)$ and persistent bleeding $(\diamond)$. Correlation of IgG level with fibrinogen $(a, d)$, prothrombin $(b, e)$ and factor $X(c, f)$.

formation $\left(R^{2}=0.21, p<0.001\right.$ and $R^{2}=0.14, p=0.002$, respectively); this was also the case for the PT $\left(R^{2}=0.12, p=0.007\right.$ and $R^{2}=0.15, p=0.001$, respectively). However, it is noted that correlation coefficients were low, and clotting times were not different between patients with persistent bleeding or stopped bleeding.

\section{Discussion}

This paper shows the results of a comparative study to the effects of dilution on two key processes in coagulation, namely capacity of thrombin generation and the ability 
of formation of fibrin clots. In study $A$, where the effect of in vitro dilution was determined of plasmas from patients treated with vitamin $\mathrm{K}$ antagonist, reduced plasma levels of coagulation factors (prothrombin, factor VII, IX, X) were accompanied by a lower thrombin generation, but not by a reduction in fibrin clot formation. The vitamin $\mathrm{K}$ antagonist prolonged the lag-times in both tests, such in agreement with the increased INR values. This effect has also been described in another study on the influence of coumarin therapy on thrombin generation [23]. The dilution of control and patient plasmas resulted in a decreased fibrin clot formation, independently of the INR value. Correspondingly, also other studies and case reports with patients undergoing fluid infusion have indicated that re-administration of fibrinogen in plasma can normalize fibrin clot formation in a way accompanied by

Table 4: Predictive variables of thrombin generation and fibrin clot formation tests.

\begin{tabular}{|c|c|c|c|c|}
\hline Variable & $\begin{array}{l}\text { (diluted) } \\
(n=84)\end{array}$ & $\begin{array}{l}\text { Study B + C } \\
\text { after CPB } \\
(n=30)\end{array}$ & $\begin{array}{l}\text { Study B } \\
\text { before FFP } \\
(n=54)\end{array}$ & $\begin{array}{l}\text { Study C } \\
\text { after FFP } \\
(n=54)\end{array}$ \\
\hline Thrombin generation $\left(R^{2}\right)$ & $0.32^{* * *}$ & 0.10 & $0.44^{* * *}$ & $0.46^{* * *}$ \\
\hline fibrinogen $(\beta)$ & ns & ns & $-0.33^{*}$ & ns \\
\hline prothrombin $(\beta)$ & ns & ns & $0.68^{* \star *}$ & ns \\
\hline factor $X(\beta)$ & $0.32^{*}$ & + & ns & $0.97^{* * *}$ \\
\hline factor XIII ( $\beta)$ & ns & ns & ns & ns \\
\hline antithrombin $(\beta)$ & ns & ns & ns & $-0.45^{*}$ \\
\hline $\lg G(\beta)$ & $0.33^{*}$ & + & ns & ns \\
\hline Fibrin clot formation $\left(R^{2}\right)$ & $0.60^{* * *}$ & $0.41^{\star \star *}$ & $0.74^{* * *}$ & $0.88^{* * *}$ \\
\hline fibrinogen $(\beta)$ & $0.78^{* * *}$ & $0.64^{* * *}$ & $0.58^{* * *}$ & $0.66^{* * *}$ \\
\hline factor $X(\beta)$ & ns & ns & ns & $0.21^{*}$ \\
\hline factor XIII ( $\beta)$ & ns & ns & $0.38^{* *}$ & ns \\
\hline antithrombin $(\beta)$ & ns & ns & ns & ns \\
\hline $\lg G(\beta)$ & + & + & ns & $0.26^{* *}$ \\
\hline
\end{tabular}

For multiple regression analysis of all indicated parameters, associations are indicated as $R^{2}$ values. For individual parameters, values are standardized $\beta$ coefficients. ns, not significant, ${ }^{+} p<0.1$ (borderline), ${ }^{*} p<0.05,{ }^{* *} p<0.01,{ }^{* * *} p<0.001$. 
stop of bleeding $[10,17,24]$. Also supplementation of a fibrinogen concentrate was found to increase fibrin clot formation $[17,25,26]$. The results of studies $B$ and $C$ provide further mechanistic insight into the hemostatic changes in dilutional coagulopathy. In general, it appeared that peri-operative bleeding was accompanied with a low thrombin peak height $(62.5-76 \%)$ or a low thromboelastography amplitude (75-76\%). Both tests were low in $50-59 \%$ of cases with bleeding, but in only $13-20 \%$ of non-bleeding cases. As the tests measure thrombin generation and fibrin clot formation in an optimized integrative way (e.g. triggered by high tissue factor), this suggests that the capacity of either process rather than the onset of it (lag-time) is linked to bleeding.

Using thromboelastography, also other authors have been able to detect hemostatic insufficiencies in whole blood from patients undergoing surgery or with trauma $[4,5]$. Here, especially the so-called FIBTEM test was found to be indicative for bleeding tendencies. In the FIBTEM modification, elastic clots are formed without a contribution of platelets [27] and, hence, this test informs on the clotting system only, which is comparable to the present measurement. On the other hand, platelets do have an important role in haemostasis, and the use of capacity coagulation tests also incorporating the function of platelets will be of additive value. Earlier work has already shown that platelets can partly compensate for the effect of moderate dilution on coagulation activity [17]. An important result of studies $B$ and $C$ is that in individual patients the processes of thrombin generation and fibrin clot formation are independently reduced, and that either process can become rate-limiting separately from the other one. This refutes the suggestion from other authors that, in coagulopathy, fibrin clot formation may be merely a reflection of the thrombin generation process $[2,28]$. In addition, our data indicate that, in diluted plasma, different coagulation factor levels control these processes, i.e. in particular factor $X$ as a main determinant of thrombin generation, and fibrinogen as the key variable of fibrin clot formation.

For diagnostic purposes, it will be important to have quick point-of-care versions of both assays. As long as such a thrombin generation test is not available, our results indicate that a control of the key coagulation factors, prothrombin, fibrinogen and factor $X$ is important. On the other hand, whether levels of these factors indeed are a useful clinical trigger, needs to be confirmed. The present data show that the combined measurement of thrombin generation and fibrin clot 
formation provides a better evaluation of the coagulopathic profile in patients with dilutional coagulopathy than either test alone. Using preset thresholds for normal values, we found that at least one of these processes was low in $88-93 \%$ of the patients with (persistent) bleeding, but in only $40-53 \%$ of the non-bleeding cases. We also studied whether IgG levels could be used as a coagulation-independent dilution marker. Interestingly, this measurement did not give much extra information, as the IgG level appeared to be highly correlated with levels of prothrombin, factor $X$ and fibrinogen.

There is a current debate on possible advantages of the use of coagulation factor concentrates in the management of peri-operative massive bleeding $[25,29$ 31]. Our data suggest that such interventions may be effective, provided they are targeted at maintaining sufficient activities of thrombin generation as well as clot formation. However, randomized clinical trials are required for establishing the real efficacy of such interventions. In summary, we conclude that, in acquired dilutional coagulopathy, the processes of thrombin generation and fibrin clot formation can be affected independently, and that sufficient activities of both are needed to prevent or stop bleeding. Both tests provide capacity-type of information and, jointly, they may be better predictors of individual increased bleeding risk than currently used routine coagulation times. 


\section{References}

1 Hemker HC, Al Dieri R, Beguin S: Thrombin generation assays: accruing clinical relevance. Curr Opin Hematol. 2004;11: 170-5.

2 Rivard GE, Brummel-Ziedins KE, Mann KG, Fan L, Hofer A, Cohen E: Evaluation of the profile of thrombin generation during the process of whole blood clotting as assessed by thrombelastography. J Thromb Haemost. 2005;3: 2039-43.

3 Ronald A, Dunning J: Can the use of thromboelastography predict and decrease bleeding and blood and blood product requirements in adult patients undergoing cardiac surgery? Interact Cardiovasc Thorac Surg. 2005;4: 456-63.

4 Reinhofer M, Brauer M, Franke U, Barz D, Marx G, Losche W: The value of rotation thromboelastometry to monitor disturbed perioperative haemostasis and bleeding risk in patients with cardiopulmonary bypass. Blood Coagul Fibrinolysis. 2008;19: 212-9.

Rugeri L, Levrat A, David JS, Delecroix E, Floccard B, Gros A, Allaouchiche B, Negrier C: Diagnosis of early coagulation abnormalities in trauma patients by rotation thrombelastography. J Thromb Haemost. 2007;5: 289-95.

Kozek-Langenecker S: Management of massive operative blood loss. Minerva Anestesiol. 2007;73: 401-15.

7 Schols SE, van der Meijden PE, van Oerle R, Curvers J, Heemskerk JW, van Pampus EC: Increased thrombin generation and fibrinogen level after therapeutic plasma transfusion: relation to bleeding. Thromb Haemost. 2008;99: 64-70.

Vanschoonbeek K, Feijge MA, Paquay M, Rosing J, Saris W, Kluft C, Giesen PL, de Maat MP, Heemskerk JW: Variable hypocoagulant effect of fish oil intake in humans: modulation of fibrinogen level and thrombin generation. Arterioscler Thromb Vasc Biol. 2004;24: 1734-40.

$9 \quad$ Hiippala ST, Myllyla GJ, Vahtera EM: Hemostatic factors and replacement of major blood loss with plasma-poor red cell concentrates. Anesth Analg. 1995;81: 360-5.

10 Fenger-Eriksen C, Lindberg-Larsen M, Christensen AQ, Ingerslev J, Sorensen B: Fibrinogen concentrate substitution therapy in patients with massive haemorrhage and low plasma fibrinogen concentrations. Br J Anaesth. 2008;101: 769-73.

Karlsson M, Ternstrom L, Hyllner M, Baghaei F, Nilsson S, Jeppsson A: Plasma fibrinogen level, bleeding, and transfusion after on-pump coronary artery bypass grafting surgery: a prospective observational study. Transfusion. 2008;48: 2152-8.

12 Rosing J, Tans G, Govers-Riemslag JW, Zwaal RF, Hemker HC: The role of phospholipids and factor Va in the prothrombinase complex. J Biol Chem. 1980;255: 274-83.

13 Vanschoonbeek K, Feijge MA, Van Kampen RJ, Kenis H, Hemker HC, Giesen PL, Heemskerk JW: Initiating and potentiating role of platelets in tissue factor-induced thrombin generation in the presence of plasma: subject-dependent variation in thrombogram characteristics. J Thromb Haemost. 2004;2: 476-84.

14 O'Shaughnessy DF, Atterbury C, Bolton Maggs P, Murphy M, Thomas D, Yates S, Williamson LM: Guidelines for the use of fresh-frozen plasma, cryoprecipitate and cryosupernatant. $\mathrm{Br} \mathrm{J}$ Haematol. 2004;126: 11-28.

Chowdhury P, Saayman AG, Paulus U, Findlay GP, Collins PW: Efficacy of standard dose and $30 \mathrm{ml} / \mathrm{kg}$ fresh frozen plasma in correcting laboratory parameters of haemostasis in critically ill patients. Br J Haematol. 2004;125: 69-73.

16 Hemker HC, Al Dieri R, De Smedt E, Beguin S: Thrombin generation, a function test of the haemostatic-thrombotic system. Thromb Haemost. 2006;96: 553-61.

17 Schols SE, Feijge MA, Lance MD, Hamulyak K, ten Cate H, Heemskerk JW, van Pampus EC: Effects of plasma dilution on tissue-factor-induced thrombin generation and thromboelastography: partly compensating role of platelets. Transfusion. 2008;48: 2384-94. van der Meijden PE, Feijge MA, Giesen PL, Huijberts M, van Raak LP, Heemskerk JW: Platelet $\mathrm{P}_{2} \mathrm{Y}_{12}$ receptors enhance signalling towards procoagulant activity and thrombin generation. A study with healthy subjects and patients at thrombotic risk. Thromb Haemost. 2005;93: 1128-36.

Mousa SA, Khurana S, Forsythe MS: Comparative in vitro efficacy of different platelet glycoprotein Ilb/llla antagonists on platelet-mediated clot strength induced by tissue factor with use of thromboelastography: differentiation among glycoprotein Ilb/llla antagonists. Arterioscl Thrombosis Vasc Biol. 2000;20: 1162-7.

20 Luddington RJ: Thrombelastography/thromboelastometry. Clin Lab Haematol. 2005;27: 81-90.

21 Butenas S, van 't Veer C, Mann KG: "Normal" thrombin generation. Blood. 1999;94: 2169-78. 
22 Vanschoonbeek K, Feijge MA, Saris WH, de Maat MP, Heemskerk JW: Plasma triacylglycerol and coagulation factor concentrations predict the anticoagulant effect of dietary fish oil in overweight subjects. J Nutr. 2007;137: 7-13.

23 Brummel KE, Paradis SG, Branda RF, Mann KG: Oral anticoagulation thresholds. Circulation. 2001;104: 2311-7.

24 Weinkove R, Rangarajan S: Fibrinogen concentrate for acquired hypofibrinogenaemic states. Transfus Med. 2008;18: 151-7.

25 Haas T, Fries D, Velik-Salchner C, Reif C, Klingler A, Innerhofer P: The in vitro effects of fibrinogen concentrate, factor XIII and fresh frozen plasma on impaired clot formation after 60\% dilution. Anesth Analg. 2008;106: 1360-5.

26 Ganter MT, Hofer CK: Coagulation monitoring: current techniques and clinical use of viscoelastic point-of-care coagulation devices. Anesth Analg. 2008;106: 1366-75.

27 Nielsen VG, Geary BT, Baird MS: Evaluation of the contribution of platelets to clot strength by thromboelastography in rabbits: the role of tissue factor and cytochalasin D. Anesth Analg. 2000;91: 35-9.

28 Fenger-Eriksen C, Ingerslev J, Tonnesen E, Sorensen B: Citrate artificially masks the haemostatic effect of recombinant factor VIla in dilutional coagulopathy. Ann Hematol. 2009;88: 255-60.

29 Samama CM: Prothrombin complex concentrates: a brief review. Eur J Anaesthesiol. 2008;25: 784-9.

30 Yasaka M, Sakata T, Naritomi H, Minematsu K: Optimal dose of prothrombin complex concentrate for acute reversal of oral anticoagulation. Thromb Res. 2005;115: 455-9.

31 Mittermayr M, Streif W, Haas T, Fries D, Velik-Salchner C, Klingler A, Oswald E, Bach C, Schnapka-Koepf M, Innerhofer P: Hemostatic changes after crystalloid or colloid fluid administration during major orthopedic surgery: the role of fibrinogen administration. Anesth Analg. 2007;105: 905-17. 


\section{Chapter 6b}

Perioperative dilutional coagulopathy treated with fresh frozen plasma and fibrinogen concentrate: a prospective randomized intervention trial

Marcus D. Lancé, Marisa Ninivaggi, Saskia E. M. Schols, Marion A. H. Feijge, Stephanie K. Oehrl, Gerhardus J. A. J. M. Kuiper, Maria Nikiforou, Marco A. E. Marcus, Karly Hamulyak, Elisabeth C. M. van Pampus, Hugo ten Cate, Johan W. M. Heemskerk 


\section{Abstract}

Background: Treatment of dilutional coagulopathy by transfusing fresh frozen plasma (FFP) remains sub-optimal. We hypothesized that partial replacement of transfused FFP by fibrinogen concentrate results in improved coagulant activity and haemostasis. This was tested in a controlled clinical intervention trial with patients experiencing massive bleeding during major surgery.

Methods: Patients undergoing major elective surgery were treated according to current protocols. When transfusion with FFP was required, patients were randomized as follows: group A received 4 units FFP; group B received 2 units FFP plus $2 \mathrm{~g}$ fibrinogen concentrate. Blood samples were taken before and after the intervention. Analysts were blinded to the treatment type.

Results: Group A (B) consisted of 21 (22) patients, in 16 (17) of whom bleeding stopped after intervention. Plasma fibrinogen increased significantly more in group $B$ $(0.57 \mathrm{~g} / \mathrm{L})$ than in group $A(0.05 \mathrm{~g} / \mathrm{L})$. However, levels of prothrombin, factors VIII, IX and $\mathrm{X}$ increased more in group $\mathrm{A}$ than in group $\mathrm{B}$. Rotational thrombelastometry (ROTEM) of whole blood and plasma revealed improved fibrin clot formation in group B but not in group A. Thrombin generation (Calibrated Automated ThrombogramCAT) in plasma increased more in group A. Principal parameters determining wholeblood thrombelastometry were the fibrinogen level and platelet count. In vitro addition of fibrinogen and prothrombin complex concentrate to pre-intervention samples restored both ROTEM and CAT parameters.

Conclusions: Partial replacement of transfused FFP by fibrinogen increases fibrin clot formation at the expense of less improved thrombin generation. Coagulation factors other than fibrinogen alone are required for full restoration of haemostasis. 


\section{Introduction}

In major elective surgery and trauma, successful treatment of massive haemorrhage remains a challenge, as bleeding is still the main cause of morbidity and mortality in these situations [1, 2]. Patients undergoing major surgery develop haemodilution, as a consequence of abundant infusion of fluids for volume resuscitation and transfusion of blood components. The result often is diminished coagulation, which further aggravates the bleeding risk. Transfusion of blood components may also lead to other complications, such as acute respiratory distress syndrome, transfusion-related lung injury, transfusion-associated circulatory overload and infections [3, 4]. Recent association studies provide indications that the use of multiple blood components reduces long-term survival after cardiac surgery [5, 6]. Accordingly, alternatives for blood component transfusion, for example the use of factor concentrates, are extensively being studied.

Current guidelines for major surgery recommend transfusion therapy with crystalloids, colloids, packed red cells and fresh frozen plasma (FFP) to prevent shock and restore the coagulation processes [7, 8]. Colloids yet affect haemostasis by disturbing fibrinogen polymerization $[9,10]$, while also the clinical efficacy of FFP in preventing peri-operative bleeding is debated [11, 12]. An interesting alternative to FFP is transfusion with fibrinogen concentrate, especially since fibrinogen is the first coagulation factor falling below a critically low level upon dilution [13]. Fibrinogen concentrates have appeared to be haemostatically active in both retrospective clinical studies and animal models [14-17]. In this regard, whole-blood rotational thrombelastometry is used as a valuable point-of-care method, monitoring improved fibrin clot formation upon elevation of plasma fibrinogen levels $[13,18]$. However, the precise indications for fibrinogen transfusion and the limitations of this intervention are still debated $[19,20]$. Recently, we reported that the processes of thrombin generation and fibrin clot formation are independently reduced during dilutional coagulopathy $[9,21]$. The implication is that full replacement of transfused FFP by fibrinogen concentrate may not result in haemostasis, as levels of other coagulation factors than fibrinogen remain too low for a critical rate of thrombin generation.

In this paper we present a first prospective randomized intervention study aimed to compare the effect of partial replacement of FFP by fibrinogen concentrate on fibrin clot formation, as measured by whole blood thrombelastometry, and thrombin generation in patients with massive bleeding undergoing major elective surgery. 


\section{Materials and Methods}

\section{Study setup}

The study was designed as a single-centre, prospective, randomized, blinded intervention trial, and was approved by the medical ethical board of the Maastricht University Medical Centre ${ }^{+}$(trial registration NL23565.068.08). Patients selected as eligible prior to surgery, gave written informed consent to participate. Selection criteria were the type of operation (cardiovascular surgery, major abdominal surgery or orthopedic surgery) and the expected duration of operation (>120 min). Exclusion criteria were age (<18 years), active HIV infection, known coagulation abnormalities, deep hypothermia with circulatory arrest, or preoperative need of transfusion.

Haemostatic therapy to stop bleeding was started, based on clinical decision. Massive bleeding was defined as prolonged blood loss of $>150 \mathrm{~mL} / \mathrm{h}$ or $>1.5$ $\mathrm{mL} / \mathrm{kg} / 20 \mathrm{~min}$, or acute blood loss of $>700 \mathrm{~mL}$ at once [7, 19, 20]. Patients with massive bleeding during or after surgery were randomized to group $A$ or $B$ in the transfusion laboratory, using a closed envelope method. Products were prepared and sent to the operation theatre, where the attending anaesthesiologist managed the transfusion. Patients of group A were transfused with 4 units FFP, those of group B with 2 units FFP plus $2 \mathrm{~g}$ fibrinogen (Haemocomplettan P $20 \mathrm{~g} / \mathrm{L}$, CSL Behring, Marburg, Germany).

Blood samples were taken directly before (baseline) and immediately after completion of the transfusion products. Heparin $(150-300 \mathrm{mg} / \mathrm{kg})$ was given to 12 out of 13 (group A) and 13 out of 14 patients (group B), who required cardiovascular surgery with extracorporeal circulation. Before taking the second blood sample, the heparin was antagonized with protamine in a ratio of $1: 1$, verified by the activated clotting time (ACT). Extra protamine was given, if the baseline ACT was not reached. In few (5) plasma samples with traces of active heparin, that is, in patients with prior heparin medication, functional measurements were performed in the presence of 10 $\mathrm{U} / \mathrm{mL}$ polybrene (Janssen, Beerse, Belgium).

Criteria for stopped bleeding after transfusion were as follows: no visual blood loss or clotting, no monitored circulatory instability, and/or no major decrease in haematocrit. When haemostasis remained insufficient, therapy was continued according to standard treatment protocols. Demographic data, type of surgery, duration of the operation, peri-operative fluid management, temperature and perioperative morbidity of the patients were all documented. 


\section{Plasma preparation}

Blood was drawn into sodium citrate (1/10 volume, $10.9 \mathrm{mM}$ f.c.). Haematological parameters were determined as described [21]. Platelet-free-plasma (PFP) was prepared by centrifuging blood samples twice at $2630 \mathrm{~g}$ for $10 \mathrm{~min}$ at room temperature. Collected plasma was aliquoted and snap-frozen at $-80^{\circ} \mathrm{C}$ until use. To prepare washed platelets, blood from a healthy donor was collected into acetatecitrate dextrose and subjected to a centrifugation procedure [21].

\section{Plasma supplementation with factor concentrates}

To study effects on thrombelastometry and thrombin generation, pre-intervention plasma samples were supplemented with fibrinogen concentrate and prothrombin complex concentrate. The concentrates were added at amounts corresponding to an expected increase in fibrinogen and prothrombin level reached by transfusion of 4 units FFP. Using a published algorithm [9], this required the addition of $0.8 \mathrm{~g} / \mathrm{L}$ fibrinogen concentrate (Haemocomplettan $\mathrm{P}$ ) and/or $0.22 \mathrm{U} / \mathrm{mL}$ prothrombin complex concentrate (Beriplex, CSL-Behring). After reconstitution, the plasmas were incubated at $37^{\circ} \mathrm{C}$ for $10 \mathrm{~min}$.

\section{Coagulation times and factor measurements}

Prothrombin time (PT) was measured by triggering plasma with tissue factor (Innovin, Dade Behring, Marburg, Germany). The activated partial thromboplastin time (aPTT) was measured with the actin-FSL kit (Dade Behring). Fibrinogen was determined with a functional assay [22]. Plasma prothrombin and factors VIII, IX and X were determined with one-stage clotting assays using the Behring coagulation system (Dade Behring). Antithrombin was measured with a chromogenic assay in the same system. Plasma heparin was assessed as anti-factor Xa activity using the Coamatic heparin test (Chromogenics, Mölndal, Sweden).

Rotational thrombelastometry was performed with whole blood and plasma using a ROTEM thromboelastometer (TEM International, Munich, Germany). Freshly obtained, citrate-anticoagulated whole blood $(300 \mu \mathrm{L})$ was activated with an optimal concentration of $10 \mathrm{pM}$ tissue factor and $\mathrm{CaCl}_{2}$. Because of late availability of the equipment, ROTEM analysis was not performed with whole blood samples from the first patients entering the study. For thrombelastometry experiments with plasma, 
snap-frozen PFP was thawed, and $300 \mu \mathrm{L}$ aliquots were supplemented with donor platelets $\left(100 \times 10^{9} / \mathrm{L}\right)$ or $4 \mathrm{mM}$ phospholipids (phosphatidyl serine, phosphatidyl choline and phosphatidyl ethanolamine, 1:3:1; mol:mol, Avanti, Alabaster AL, USA). Activation was with $10 \mathrm{pM}$ tissue factor and $\mathrm{CaCl}_{2}$ [17]. Evaluation parameters of the curves were the clotting time (CT), clot formation time (CFT), maximal clot formation (MCF) and maximal rate of clot formation ( $\alpha$-angle). The two latter inform on the maximal elastic clot strength and the rate of fibrin strand formation, respectively [26]. Normal value of the MCF of PFP with phospholipids was $42 \pm 5.7 \mathrm{~mm}$ (TEG, mean \pm $\mathrm{SD}, n=40$ ); the preset cut-off level of normal fibrin clot formation was $13.5 \mathrm{~mm}$ [9].

\section{Thrombin generation measurement}

Thrombin generation was measured using the calibrated automated thrombogram method (CAT; Thrombinoscope, Maastricht, the Netherlands), with slight modifications [17, 21]. Citrate-anticoagulated PFP was supplemented with donor platelets $\left(100 \times 10^{9} / \mathrm{L}\right)$ or phospholipids $(4 \mu \mathrm{M})$, and activated with $10 \mathrm{pM}$ tissue factor plus $\mathrm{CaCl}_{2}$. Thrombin activity at $37^{\circ} \mathrm{C}$ was determined from the cleavage of thrombin substrate, Z-Gly-Gly-Arg aminomethyl coumarin (Z-GGR-AMC, Bachem, Bubendorf, Switzerland). Samples were run in triplicate. Evaluated curve parameters were the thrombin peak height and endogenous thrombin potential (ETP, area-under-thecurve) [27]. The intra-assay variation coefficient was $6 \%$. Normal value of the thrombin peak height in PFP with phospholipids was $314 \pm 24 \mathrm{nM}$ (mean \pm SD, $n=40$ ); the cut-off level for normal thrombin generation was $194 \mathrm{nM}$ [9].

\section{Statistical analysis}

Data are expressed as means \pm SD. Data from study groups were compared by ANOVA. Treatment effects on assay parameters (normally distributed) were assessed with a paired t-test (2-tailed) and Pearson correlation analysis. The statistical package for the social sciences was used (SPSS, version 15.0).

\section{Results}

During the study period of 17 months, 307 eligible patients gave informed consent prior to surgery. During surgery, 255 did not meet the predefined criteria for massive bleeding. Of the remaining 52 patients with bleeding, 9 patients required transfusion of only 2 units FFP and hence were not included (Fig. 1S). The remaining 43 patients 


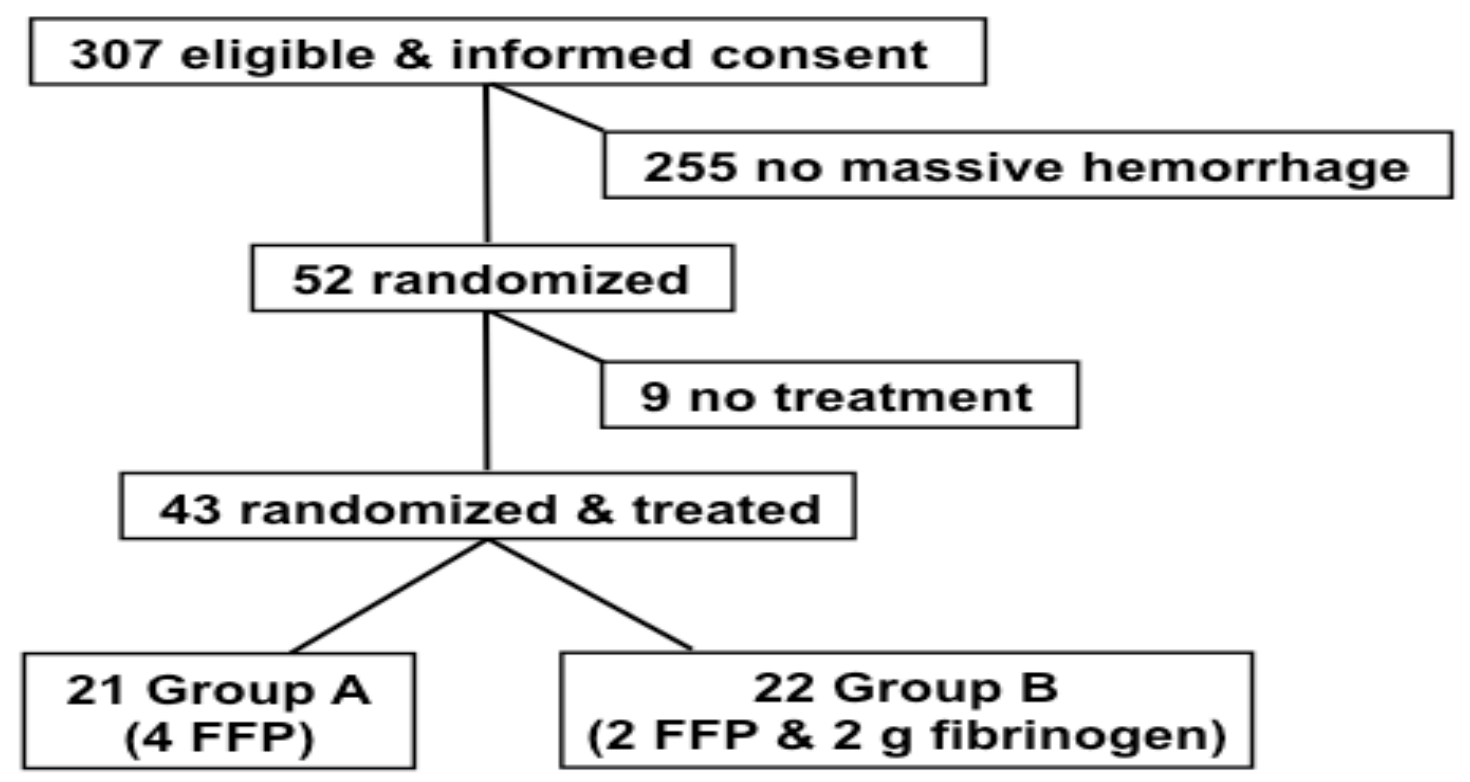

Figure 1S: Patient inclusion and randomization

with massive bleeding entered the study protocol, and received either 4 units FFP (group $\mathrm{A}$ ) or 2 units FFP plus $2 \mathrm{~g}$ fibrinogen (group B).

Group A consisted of 21 patients ( $15 \mathrm{men} / \mathrm{six}$ women) with mean age of 65.2 years; group B contained 22 patients (16 men/six women) with mean age of 65.6 years (Table 1). In either group, the majority of patients underwent cardiovascular surgery (13 and 14 , respectively), while the other patients had abdominal ( 7 and 6 ) or spinal column surgery ( 1 and 2). Patients of groups $A$ and $B$ did not differ with respect to the length of operation. The volume of infused colloids (HES 6\% 130/04) was similar for both groups. Also the total fluid volume transfused prior to intervention was similar: $8.51 \pm 2.64 \mathrm{~L}$ for group $A$ and $8.72 \pm 4.13 \mathrm{~L}$ for group $B$ (mean $\pm S D$ ).

After intervention, haemostasis was sufficient in 16 patients of group $A$ and 17 of group $B(p=0.93)$. In the remaining patients with continued bleeding, five per group, prohaemostatic treatment was continued according to the standard hospital protocol. One patient with bleeding in either group showed pleural effusion, 3 patients of group A had wound infections (one associated with deep vein thrombosis), while in group B, one patient had wound infections, two had septic complications (one pneumonia treatment), and one abdominal ischemia needing surgical revision.

Regarding haematological parameters, haemoglobin and platelet count were similar in both groups, without significant changes after intervention (Table 2). 
Table 1: Patient and surgery characteristics of groups A and B.

\begin{tabular}{lcc}
\hline & Group A & Group B \\
\cline { 2 - 3 } Male/female $(n)$ & $15 / 6$ & $16 / 6$ \\
Age (years) & $65.2(9.8)$ & $65.6(10.7)$ \\
Surgery type: $(n)$ & & \\
$\quad$ cardiovascular & $13^{*}$ & $14^{*}$ \\
abdominal & 7 & 6 \\
$\quad$ spinal column & 1 & 2 \\
Surgery duration (min) & $374(126)$ & $355(96)$ \\
Temperature pre-intervention $\left({ }^{\circ} \mathrm{C}\right)$ & $35.9(1.2)$ & $35.8(1.1)$ \\
Transfusion before intervention: & & \\
$\quad$ packed red cells (units) & $6.33(2.80)$ & $5.86(3.54)$ \\
$\quad$ colloid HES 130/0.4 (L) & $1.89(0.89)$ & $2.00(0.87)$ \\
total fluid volume $(\mathrm{L})$ & $8.51(2.64)$ & $8.72(4.13)$
\end{tabular}

Total fluids transfused prior to intervention comprise colloids, crystalloids and priming and cell saver fluids. Data are means (SD). *12/13 patients (group A) and 14/14 patients (group B) received $3 \mathrm{~g}$ tranexamic acid.

Table 2: Hematological and coagulation parameters of patients pre- and post-intervention.

\begin{tabular}{rrrrrrr}
\hline & \multicolumn{3}{c}{ Group A (n=21) } & \multicolumn{3}{c}{ Group B $(n=22)$} \\
& pre & post & $\Delta$ & pre & post & \multicolumn{1}{c}{$\Delta$} \\
\hline $\mathrm{Hb}(\mathrm{mM})$ & $5.3(1.3)$ & $5.2(1.3)$ & -0.09 & $5.5(1.3)$ & $5.5(1.7)$ & +0.01 \\
$\mathrm{Ht}(\%)$ & $0.26(0.06)$ & $0.24(0.06)$ & -0.02 & $0.26(0.06)$ & $0.27(0.06)$ & +0.01 \\
Platelets (10\%) & $108(56)$ & $97(43)$ & -12 & $83(42)$ & $82(47)$ & -1 \\
aPTT (s) & $96(68)$ & $45(19)$ & $-49 *$ & $93(48)$ & $70(40)$ & -24 *\# \\
PT (s) & $23(30)$ & $13(2)$ & -9.5 & $25(30)$ & $15(2)$ & -10 \\
Fibrinogen (g/L) & $1.59(0.97)$ & $1.64(0.66)$ & +0.06 & $1.22(0.58)$ & $1.79(0.61)$ & +0.57 *\# \\
Prothrombin (\%) & $36.8(16.8)$ & $52.1(18.2)$ & $+15.3 * 31.4(13.5)$ & $36.6(13.4)$ & +5.2 *\# \\
Factor VIII (\%) & $61.4(40.3)$ & $85.3(33.9)$ & $+23.9 * 75.9(39.5)$ & $80.2(43.3)$ & $+4.3^{\#}$ \\
Factor IX (\%) & $50.1(27.5)$ & $67.4(26.4)$ & $+17.3 * 52.3(30.4)$ & $57.8(27.4)$ & +5.8 \\
Factor X (\%) & $35.2(15.7)$ & $50.3(17.9)$ & $+15.0 *$ & $32.5(14.4)$ & $37.8(13.8)$ & 5.3 *\#\# \\
Antithrombin (\%) & $41.8(13.9)$ & $55.0(16.8)$ & $+13.1 * 38.9(12.7)$ & $42.9(13.7)$ & 4.0 *\# \\
\hline
\end{tabular}

Data are means (SD); mean differences due to intervention are indicated $\Delta .{ }^{*} p<0.05 \mathrm{vs}$. preintervention, ${ }^{\#} p<0.05,{ }^{\#} p<0.01$ vs. group A. 
Plasmas from both groups showed a prolongation of the aPTT, but not the PT. After intervention, the aPTT significantly shortened more in group A than in group B $(p=0.043)$, while fibrinogen levels increased only in group B. Plasma levels of prothrombin, factors VIII, IX and $\mathrm{X}$ and antithrombin increased more in group $\mathrm{A}$ than in group B (Table 2).

Table 3: Thrombelastometry (ROTEM) parameters.

\begin{tabular}{|c|c|c|c|c|c|c|}
\hline & \multicolumn{3}{|c|}{ Group A $(n=21)$} & \multicolumn{3}{|c|}{ Group B $(n=22)$} \\
\hline & pre & post & $\Delta$ & pre & post & $\Delta$ \\
\hline \multicolumn{7}{|c|}{ ROTEM (whole blood) ${ }^{\$}$} \\
\hline CT (s) & $262(287)$ & $176(83)$ & -86 & $431(448)$ & 233 (158) & $-198 * *$ \\
\hline CFT (s) & $535(641)$ & 258 (289) & -277 & $609(375)$ & $372(293)$ & $-237^{*}$ \\
\hline $\operatorname{MCF}(\mathrm{mm})$ & $40.0(13.6)$ & $43.4(11.1)$ & +3.4 & $32.2(12.4)$ & $39.9(14.7)$ & $+7.7 * * \#$ \\
\hline Alpha $\left(^{\circ}\right)$ & $48.3(20.2)$ & $56.0(13.5)$ & $+7.7^{*}$ & $34.8(15.6)$ & $46.7(17.6)$ & $+11.9 * *$ \\
\hline \multicolumn{7}{|c|}{ ROTEM (PRP) } \\
\hline CT (s) & $138(59)$ & $107(17)$ & $-31 *$ & $173(122)$ & $145(101)$ & -28 ** \\
\hline CFT (s) & $131(82)$ & $89(30)$ & $-42 * *$ & $145(102)$ & $110(62)$ & $-36 *$ \\
\hline $\operatorname{MCF}(\mathrm{mm})$ & $57.1(12.2)$ & $60.4(6.1)$ & +3.3 & $54.4(10.6)$ & $59.7(6.7)$ & $+5.3^{* *}$ \\
\hline Alpha $\left(^{\circ}\right)$ & $67.0(10.3)$ & $72.5(5.7)$ & $+5.5 *$ & $66.8(11.9)$ & $71.3(7.3)$ & $+4.5^{*}$ \\
\hline \multicolumn{7}{|c|}{ ROTEM (PFP) } \\
\hline CT (s) & $232(212)$ & $116(50)$ & $-117^{*}$ & $216(245)$ & $141(152)$ & $-75^{* *}$ \\
\hline $\mathrm{MCF}(\mathrm{mm})$ & $12.6(8.3)$ & $13.6(7.7)$ & +1.0 & $9.6(6.0)$ & $14.9(6.2)$ & $+5.3 * * \#$ \\
\hline Alpha $\left(^{\circ}\right)$ & $29.3(30.7)$ & $44.8(29.8)$ & +15.5 & $22.0(25.7)$ & $51.7(27.7)$ & $+29.7^{* *}$ \\
\hline
\end{tabular}

ROTEM was performed with whole blood, reconstituted PRP or PFP plus phospholipids in samples collected pre- and post-intervention. Data are means (SD). Mean differences due to intervention are indicated by $\Delta$. In all assays, coagulation was triggered with tissue factor. Analyzed curve parameters were clotting time (CT), clot formation time (CFT), maximal clot firmness (MCF) and a-angle (alpha). ${ }^{\$} N=16,{ }^{*} p<0.05,{ }^{* *} p<0.01$ vs. pre-intervention, ${ }^{\#} p<0.05$ vs. group $\mathrm{A}$.

Samples of 32 patients were used for whole-blood thrombelastometry (ROTEM). Fibrin clot formation curves for group $B$, but not for group $A$, showed an improvement after intervention of all parameters, including the MCF and $\alpha$-angle (Table 3). The intervention effect on MCF was significantly higher in group B (Fig. 1A). Thrombelastometry (ROTEM) was then performed with plasma samples from all patients in the presence of donor platelets (reconstituted platelet rich plasma - PRP) or PFP plus phospholipid vesicles. The same trends were observed for 
thrombelastometry curves with either platelets or phospholipid vesicles present (Table 3). The intervention effect on MCF once more was higher in group B (Fig. 1B, C), which corresponded to the more increased fibrinogen level in group B plasmas (Fig. 1D). Thrombin generation was measured with the CAT assay, again using reconstituted PRP or PFP with phospholipid vesicles and triggering by 10 pM tissue factor. Of the two key curve parameters, that is, thrombin peak height and endogenous thrombin potential (ETP), the former was most sensitive to the interventions (Table 4).

The thrombin peak height after intervention increased more in group $A$ than in group B (Fig. 1E, F). In comparison to previously established cut-off values for normal thromboelastography and thrombin generation in PFP [9], at least one of these values was sub-threshold in 15/21 (71\%) and 20/22 (91\%) of pre-intervention plasmas from groups $A$ and $B$, respectively.

A Rotem (whole blood)

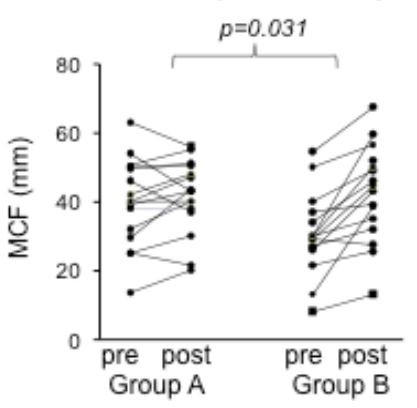

D Fibrinogen

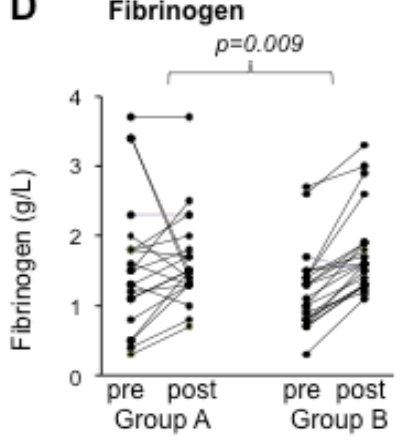

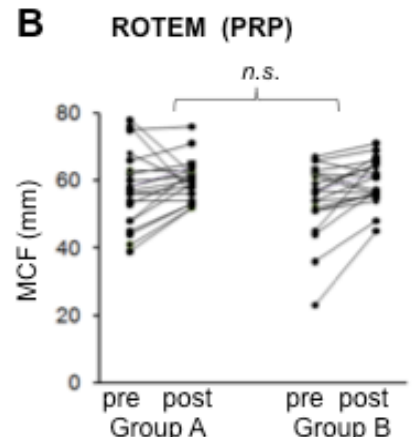

C Rotem (PFP)

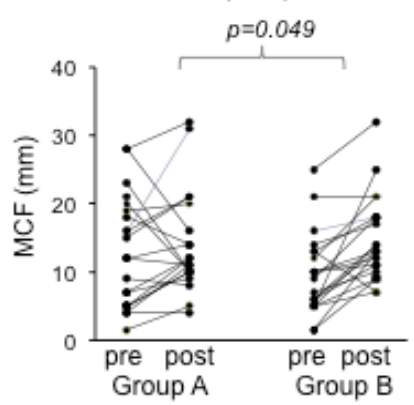

E

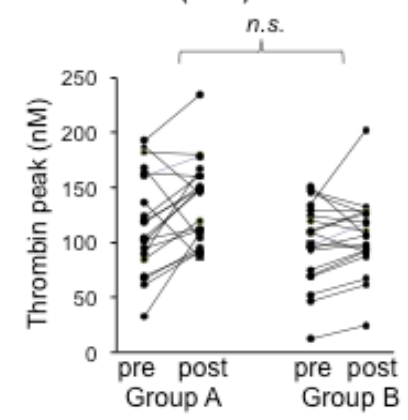

F CAT (PFP)

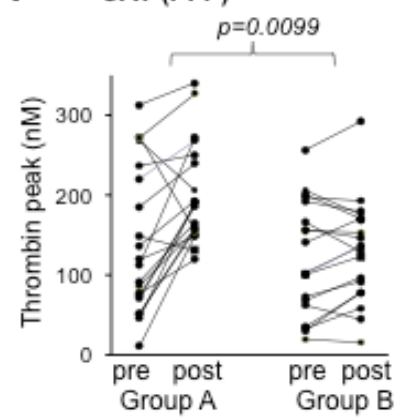

Figure 1: Intervention effects on various coagulation parameters in groups A and B. Indicated are per patient group data measured in pre- and post-intervention samples of: MCF of ROTEM in whole-blood (A); MCF in reconstituted PRP (B); MCF in PFP with phospholipids (C); fibrinogen level (D); thrombin peak height of CAT in reconstituted PRP (E) and PFP with phospholipids (F). Significance is shown of difference of treatment effect $(\Delta)$ between groups. For mean values, see Tables 3 and 4 . 
Table 4: Thrombin generation assay (CAT) parameters.

\begin{tabular}{lcccccc}
\hline & \multicolumn{3}{c}{ Group A $(n=21)$} & \multicolumn{3}{c}{ Group B $(n=22)$} \\
\cline { 2 - 7 } & pre & post & $\Delta$ & pre & post & $\Delta$ \\
\hline CAT (PRP) & & & & & \\
Peak height (nM) & $118(46)$ & $137(38)$ & $+18^{*}$ & $99(35)$ & $111(40)$ & +12 \\
ETP (nM×min) & $1134(414)$ & $1193(358)$ & +59 & $1030(323)$ & $1022(262)$ & -8 \\
CAT (PFP) & & & & & & \\
Peak height (nM) & $142(92)$ & $200(63)$ & $+58^{*}$ & $117(71)$ & $131(63)$ & +14 \\
ETP (nM×min) & $993(431)$ & $1114(352)$ & $+121^{*}$ & $899(322)$ & $886(243)$ & -13
\end{tabular}

CAT was performed with reconstituted PRP or PFP plus phospholipids in samples collected pre- and post-intervention. Coagulation was triggered with tissue factor. Data are means (SD). Mean differences due to intervention are indicated by $\Delta$. Curve parameters analyzed were thrombin peak height and endogenous thrombin potential (ETP). ${ }^{*} p<0.05$ vs. pre-intervention, ${ }^{\#} p<0.01$ vs. group $A$.

Regression analysis of the data from both groups combined pointed to significant correlations between whole-blood MCF values, on the one hand, and platelet count, fibrinogen level and prothrombin level on the other hand (Fig. 2A-C). The MCF of whole blood also correlated with the plasma MCF (Fig. 2D), which in turn correlated with the fibrinogen level (Fig. 2E). Thrombin peak height in CAT assays furthermore correlated with prothrombin (Fig. 2F). In all correlation plots, results from groups $A$ and $B$ were equally distributed. 
A

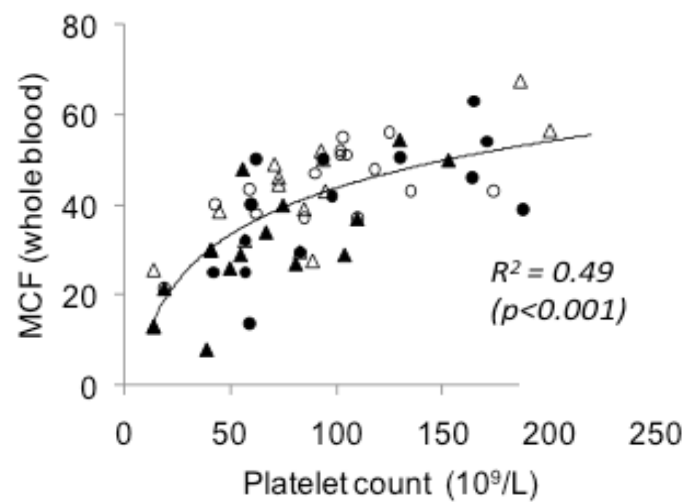

C

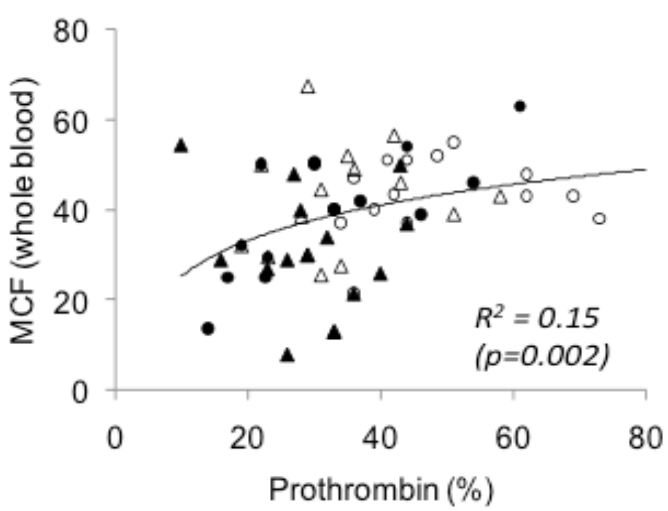

E

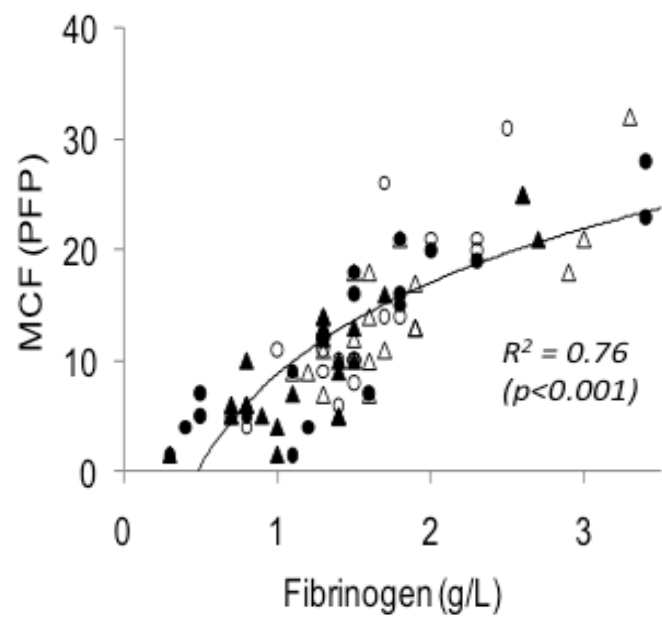

B

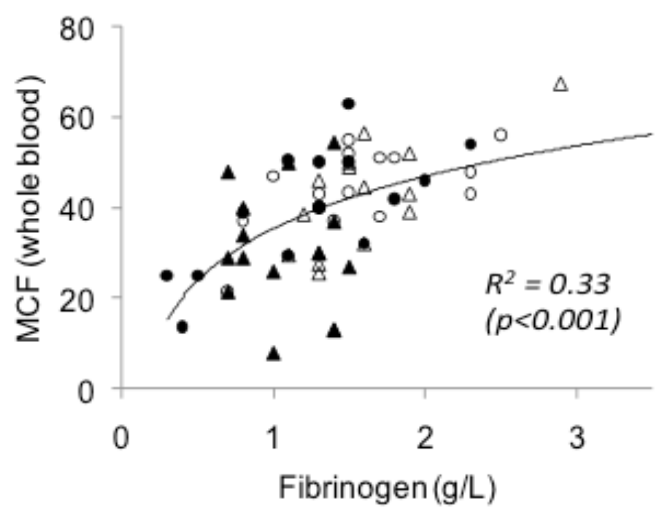

D

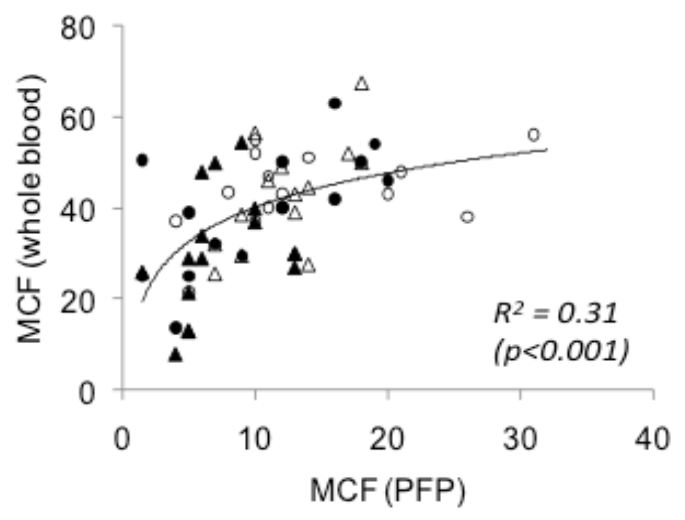

$\mathbf{F}$

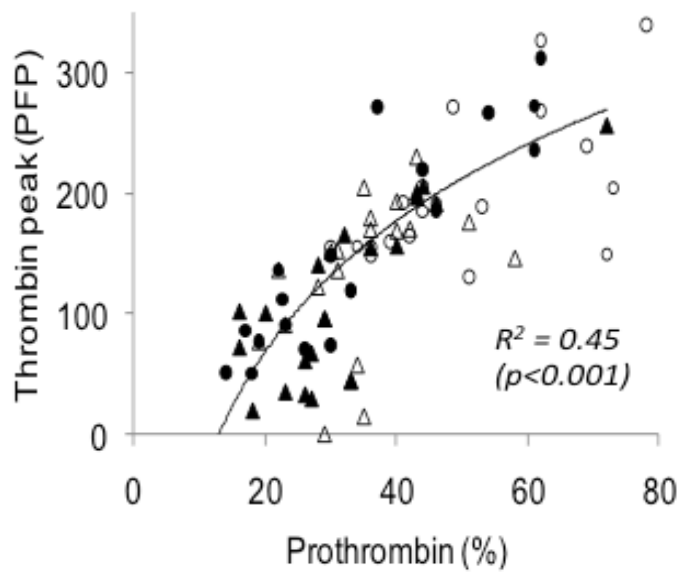

Figure 2: Correlation of thrombelastometry and thrombin generation with various parameters before and after intervention. Regression analysis of whole-blood MCF (ROTEM) versus platelet count (A), fibrinogen level (B), prothrombin level (C), and MCF in plasma (D). Regression analysis of MCF in plasma (ROTEM) with fibrinogen level (E), and of thrombin peak height (CAT) with prothrombin level (F). Data from group A are indicated by bullets, those from group B by triangles (closed = preintervention, open = post-intervention). 
More detailed multiple regression analysis indicated that the correlations between whole-blood MCF and platelet count or fibrinogen level remained significant $(p<0.001)$, when the prothrombin level was taken as control variable (i.e. as surrogate marker of plasma dilution). However, the correlation between whole-blood MCF and prothrombin level disappeared $(p=0.77)$ after normalization for platelet count and fibrinogen level. The MCF in plasma samples correlated with thrombin peak height in the presence $(p=0.009)$ or absence $(p=0.027)$ of platelets. Thrombin peak height furthermore correlated with most coagulation factors. Together, this indicated that key determinants of whole-blood thromboelastometry, measuring fibrin clot formation, are the platelet count and fibrinogen level.

Knowing that in dilutional coagulopathy the low levels of fibrinogen and other coagulation factors result in sub-normal thromboelastometry and thrombin generation [17], we investigated the effect of in vitro supplementation of fibrinogen and prothrombin complex concentrate to pre-intervention patient plasmas. Samples were incubated with both factor concentrates, at amounts corresponding to the expected increase in fibrinogen and prothrombin after transfusion of 4 units FFP, that is, 0.8 $\mathrm{g} / \mathrm{L}$ fibrinogen concentrate and $0.22 \mathrm{U} / \mathrm{mL}$ prothrombin complex concentrate [9]. Because of the limited amounts of plasma presence, in vitro addition was only performed with both concentrates together. For thromboelastometry assays in PFP, addition of factor concentrates markedly improved the MCF and $\alpha$-angle (Fig. 3A, B). This corresponded to the intervention effect in groups $A$ and $B$. In the already high thromboelastometry curves with reconstituted PRP, no extra effect of factor concentrate addition was seen. On the other hand, in CAT curves with PFP or reconstituted PRP, factor concentrate addition resulted in increased values of both thrombin peak height and ETP (Fig. 3C, D), thus resembling the intervention effect on thrombin generation in group $A$ only. 

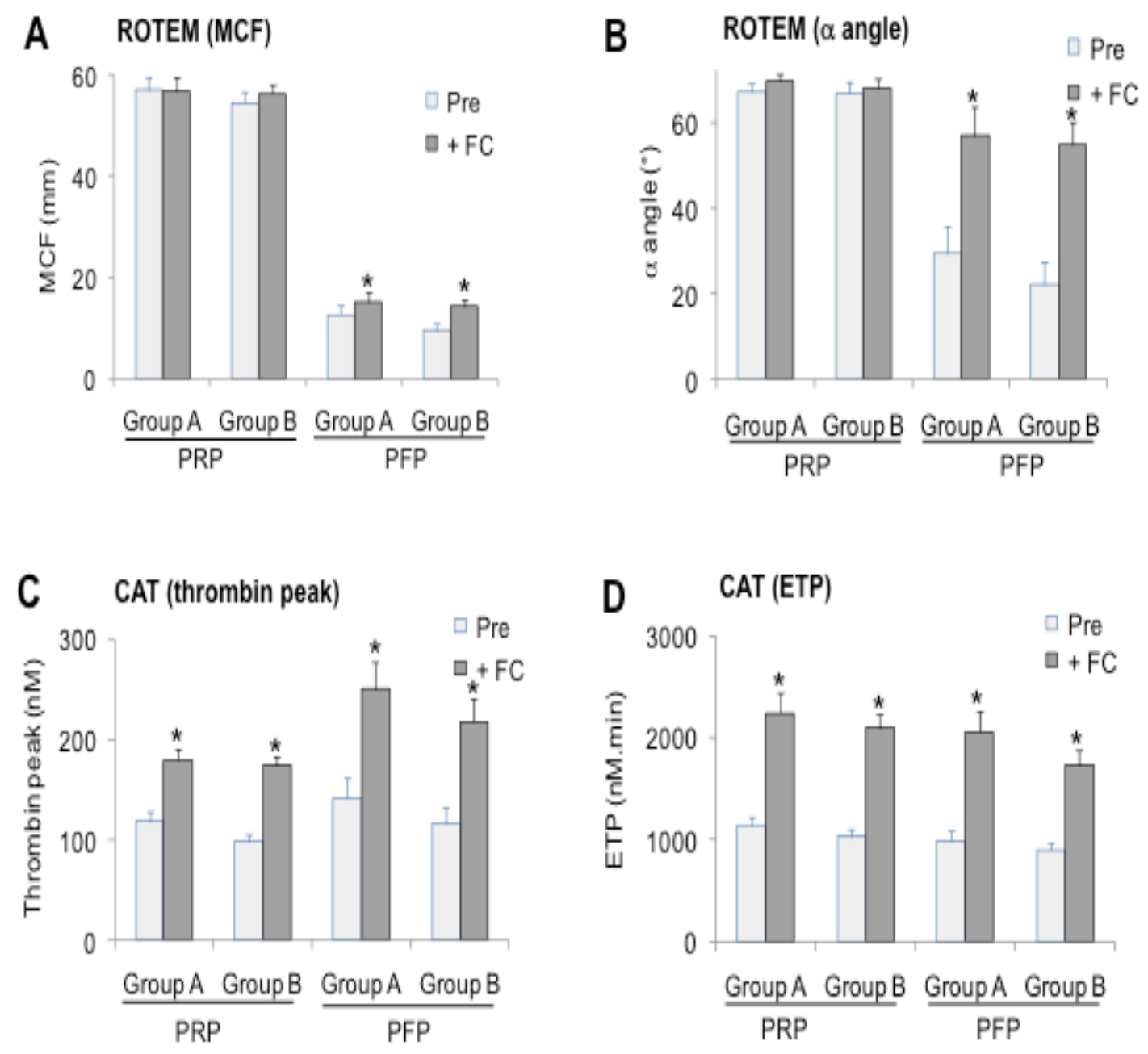

Figure 3: Effects of addition of factor concentrates (FC) on thrombelastometry and thrombin generation. Pre-intervention plasma samples were reconstituted with $0.8 \mathrm{~g} / \mathrm{L}$ fibrinogen concentrate and/or $0.22 \mathrm{U} / \mathrm{mL}$ prothrombin complex concentrate. Thromboelastometry (ROTEM) and thrombin generation (CAT) were measured in reconstituted PRP or PFP with phospholipid vesicles, after triggering with $10 \mathrm{pM}$ tissue factor. Means $\pm \operatorname{SEM}(n=20-21),{ }^{*} p<0.05$. 


\section{Discussion}

This study describes a first prospective, randomized clinical trial, comparing the prohaemostatic effects of partial replacement of FFP transfusion by fibrinogen concentrate in a total of 43 patients with massive haemorrhage. In the patients of group A, receiving 4 units FFP, levels of coagulation factors (with the exception of fibrinogen) were more increased than in group $B$, which translated to a more improved thrombin generation profile. Conversely, in the patients of group $B$, who received 2 units FFP plus $2 \mathrm{~g}$ fibrinogen, plasma fibrinogen was more increased, which translated into higher thromboelastometry curves. Hence, by partial replacement of FFP by fibrinogen, the improved capacity of fibrin clot formation was accompanied by a reduced normalization of thrombin generation. In group A 16 out of 21 and in group B 17 out of 22 stopped bleeding, while in either treatment arm 5 patients continued to bleed. Limitations of the study were the relatively small sample size with patients undergoing various types of surgery, and the fact that inclusion was based on clinical decision with the attending anaesthesiologist not blinded to the study intervention.

The CAT assay effectively detected a better improvement in coagulant activity, if tested in the absence of platelets, after intervention with 4 units FFP in comparison to 2 units FFP plus $2 \mathrm{~g}$ fibrinogen. Comparison of the curve parameters indicated that the thrombin peak height was a more sensitive marker than the ETP. The likely reason for this is that the ETP (area-under-the-curve) is substantially influenced by the calculated level of curve ending, i.e. the calculated $\alpha_{2 \mathrm{M}}$-macroglobulin correction [28]. Under conditions of hemodilution, this correction may need to be made in a nonstandard way. The thrombin peak height, though, correlated well with the plasma levels of prothrombin and other coagulation factors.

On the other hand, transfusion of $2 \mathrm{~g}$ fibrinogen plus 2 units FFP resulted in a more active fibrin clot formation, as determined by thrombelastometry (ROTEM) in whole blood and plasma. In agreement with this, we found that, next to the platelet count, the fibrinogen level is the main determining variable of whole-blood thrombelastometry, which has also been observed by others [13]. In our study, transfusion with only FFP was less effective in increasing plasma fibrinogen than cotransfusion of fibrinogen concentrate. While these data are in agreement with recent surveys $[11,12]$, they also give support to the proposal of using a high ratio of FFP to red cells in treatment of massive hemorrhage [29,30]. 
We chose to administer a low dose of $2 \mathrm{~g}$ fibrinogen to minimize possible risks for thrombosis. This choice was supported by retrospective investigations, indicating that a single dose of $2 \mathrm{~g}$ fibrinogen supplemental to FFP and platelets was effective in resolving hemorrhage [31]. In some clinical centers, fibrinogen is infused at higher amounts up to $14 \mathrm{~g}$ to reach plasma concentrations above 1.5-2 g/L [32]. Also in porcine models, higher doses of fibrinogen and prothrombin complex concentrate have been employed to arrest surgical bleeding [33, 34]. Although the two intervention regimes stopped bleeding in the majority of the patients, hemostasis was insufficient in 5 patients of each group. Sub-analysis of the coagulation profile in the patients with persistent bleeding indicated on average low levels of fibrinogen ( $A$ : bleeding and non-bleeding, $1.3 \pm 0.4$ and $1.7 \pm 0.4$; $\mathrm{B}$ : bleeding and non-bleeding, $1.5 \pm 0.6$ and $1.8 \pm 0.6 \mathrm{~g} / \mathrm{L}$; mean $\pm \mathrm{SD}$ ) and of prothrombin (A: $43 \pm 10$ and 55 $\pm 19 \%$; $B$ : $33 \pm 12$ and $36 \pm 14 \%$, respectively). Furthermore, the majority of patients with persistent bleeding were low in the previously determined [9] threshold values of normal thrombin peak height (A: $5 / 5, B: 5 / 5$ patients) and of normal MCF of fibrin clot formation (A: 4/5, B: $3 / 5$ patients). Hence, we inferred that insufficient coagulant activity due to low levels of fibrinogen as well as other coagulation factors was a main cause of persistent bleeding. Hence, the present findings are in support of the earlier conclusion that combined testing of thrombelastometry and thrombin generation can better predict peri-operative bleeding in major surgery than either assay alone [9].

In conclusion, this trial showed a similar effect of $2 \mathrm{~g}$ fibrinogen additive to 2 units FFP compared with transfusion of 4 units FFP on hemostasis. The transfused fibrinogen had an important contribution to fibrin clot formation in thrombelastometry analysis, while the transfused 4 units FFP more increased thrombin generation and fibrinogen levels remained lower. We hence postulate that transfused FFP and fibrinogen are both relevant for early hemostasis and that both should be combined in massive hemorrhage protocols. These results argue for a larger-scale follow-up study, where the effects are determined of supplementing fibrinogen concentrate together with a standard dose of FFP. Herein, a prohemostatic effect of fibrinogen is expected on top of the effect of normalization of other coagulation factors. 


\section{References}

1 Sauaia A, Moore FA, Moore EE, Moser KS, Brennan R, Read RA, Pons PT: Epidemiology of trauma deaths: a reassessment. J Trauma. 1995;38: 185-93.

2 Cothren CC, Moore EE, Hedegaard HB, Meng K: Epidemiology of urban trauma deaths: a comprehensive reassessment 10 years later. World J Surg. 2007;31: 1507-11.

3 Norda R, Tynell E, Akerblom O: Cumulative risks of early fresh frozen plasma, cryoprecipitate and platelet transfusion in Europe. J Trauma. 2006;60: S41-5.

4 Watson GA, Sperry JL, Rosengart MR, Minei JP, Harbrecht BG, Moore EE, Cuschieri J, Maier RV, Billiar TR, Peitzman AB: Fresh frozen plasma is independently associated with a higher risk of multiple organ failure and acute respiratory distress syndrome. J Trauma. 2009;67: 221-7.

5 Murphy GJ, Reeves BC, Rogers CA, Rizvi SI, Culliford L, Angelini GD: Increased mortality, postoperative morbidity, and cost after red blood cell transfusion in patients having cardiac surgery. Circulation. 2007;116: 2544-52.

6 Marik PE, Corwin HL: Efficacy of red blood cell transfusion in the critically ill: a systematic review of the literature. Crit Care Med. 2008;36: 2667-74.

7 Rossaint R, Bouillon B, Cerny V, Coats TJ, Duranteau J, Fernandez-Mondejar E, Hunt BJ, Komadina R, Nardi G, Neugebauer E, Ozier Y, Riddez L, Schultz A, Stahel PF, Vincent JL, Spahn DR: Management of bleeding following major trauma: an updated European guideline. Crit Care. 2010;14: R52.

8 Thomas D, Wee M, Clyburn P, Walker I, Brohi K, Collins P, Doughty H, Isaac J, Mahoney PM, Shewry L: Blood transfusion and the anaesthetist: management of massive haemorrhage. Anaesthesia. 2010;65: 1153-61.

9 Schols SE, Lance MD, Feijge MA, Damoiseaux J, Marcus MA, Hamulyak K, ten Cate H, Heemskerk JW, van Pampus EC: Impaired thrombin generation and fibrin clot formation in patients with dilutional coagulopathy during major surgery. Thromb Haemost. 2010;103: 318328.

10 Mittermayr M, Streif W, Haas T, Fries D, Velik-Salchner C, Klingler A, Innerhofer P: Effects of colloid and crystalloid solutions on endogenous activation of fibrinolysis and resistance of polymerized fibrin to recombinant tissue plasminogen activator added ex vivo. $\mathrm{Br} \mathrm{J}$ Anaesth. 2008;100: 307-14.

11 Stanworth SJ, Brunskill SJ, Hyde CJ, McClelland DB, Murphy MF: Is fresh frozen plasma clinically effective? A systematic review of randomized controlled trials. $\mathrm{Br} \mathrm{J}$ Haematol. 2004;126: 139-52.

12 Roback JD, Caldwell S, Carson J, Davenport R, Drew MJ, Eder A, Fung M, Hamilton M, Hess JR, Luban N, Perkins JG, Sachais BS, Shander A, Silverman T, Snyder E, Tormey C, Waters J, Djulbegovic B: Evidence-based practice guidelines for plasma transfusion. Transfusion. 2010;50: 1227-39.

13 Fenger-Eriksen C, Jensen TM, Kristensen BS, Jensen KM, Tonnesen E, Ingerslev J, Sorensen B: Fibrinogen substitution improves whole blood clot firmness after dilution with hydroxyethyl starch in bleeding patients undergoing radical cystectomy: a randomized, placebo-controlled clinical trial. J Thromb Haemost. 2009;7: 795-802.

14 Grottke O, Braunschweig T, Henzler D, Coburn M, Tolba R, Rossaint R: Effects of different fibrinogen concentrations on blood loss and coagulation parameters in a pig model of coagulopathy with blunt liver injury. Crit Care. 2010;14: R62.

15 Mercier FJ, Bonnet MP: Use of clotting factors and other prohemostatic drugs for obstetric hemorrhage. Curr Opin Anaesthesiol. 2010;23: 310-6.

16 Rahe-Meyer N, Solomon C, Winterhalter M, Piepenbrock S, Tanaka K, Haverich A, Pichlmaier M: Thromboelastometry-guided administration of fibrinogen concentrate for the treatment of excessive intraoperative bleeding in thoracoabdominal aortic aneurysm surgery. J Thorac Cardiovasc Surg. 2009;138: 694-702.

17 Schols SE, Feijge MA, Lance MD, Hamulyak K, ten Cate H, Heemskerk JW, van Pampus EC: Effects of plasma dilution on tissue-factor-induced thrombin generation and thromboelastography: partly compensating role of platelets. Transfusion. 2008;48: 2384-94.

18 Weiss G, Lison S, Spannagl M, Heindl B: Expressiveness of global coagulation parameters in dilutional coagulopathy. Br J Anaesth.105: 429-36.

19 Hardy JF, de Moerloose P, Samama CM: Massive transfusion and coagulopathy: pathophysiology and implications for clinical management. Can J Anaesth. 2006;53: S40-58.

20 Bormanis J: Development of a massive transfusion protocol. Transfus Apher Sci. 2008;38: 5763. 
21 Vanschoonbeek K, Feijge MA, van Kampen RJ, Kenis H, Hemker HC, Giesen PL, Heemskerk $\mathrm{JW}$ : Initiating and potentiating role of platelets in tissue factor-induced thrombin generation in the presence of plasma: subject-dependent variation in thrombogram characteristics. $J$ Thromb Haemost. 2004;2: 476-84.

22 Clauss A: [Rapid physiological coagulation method in determination of fibrinogen]. Acta Haematologica. 1957;17: 237-46. 


\section{Chapter 7}

General discussion 
The overall aim of this thesis is to come to a better performance of the circle of quality improvement in bleeding management (Figure 1).

A general aspect is the increasingly importance of quality management in the evolving health care environment, especially given the advanced age, the comorbidities of the many of the patients and the higher complexity of surgery leading to massive hemorrhage, which has a huge impact on costs in healthcare. In contrast, the increase in resources is not holding pace with the increase in costs. One of the solutions for managing quality and costs is to standardize procedures in health care. Acute, massive hemorrhage contributes to mortality and morbidity in trauma, cardiac surgery, general surgery and obstetrical emergencies. About $40 \%$ of trauma related deaths are associated with hemorrhage [1]. On the other hand 40 to $90 \%$ of the cardiac surgical patients receive blood, which is about $10-15 \%$ of all annual transfused blood-products in elective surgery [2-4]. In the obstetric population about 12-25\% of all maternal deaths are associated with excessive blood loss [5, 6]. In contrast up to $68 \%$ postpartal transfusions are not guideline-conform and maybe even inappropriate, which means these women frequently are over-transfused [7]. As transfusion of blood products per se is related with unwanted reactions and worse outcome in general, one way to improve treatment strategies is to search for alternatives and reduce transfusion requirements [8-11]. Finally, bleeding followed by transfusion of blood products contributes to a substantial amount of hospital costs. Reduction of costs can be accomplished by a continuous circle of improvement in the management of hemorrhage [12, 13].

In various parts of this thesis, it is emphasized that the quality improvement of treatment particularly relies on the close collaboration between caregivers at the bedside and other professionals in the hospital laboratory. As indicated in chapter $\mathbf{1}$, new, promising laboratory tests have been developed that are or may be used in a point-of-care setting with whole blood without the need to prepare plasma samples. How these laboratory tests can be used in most effective and discriminative ways for optimal patient care has been studied in the ensuing chapters 2-6. Below, the major findings of these studies are discussed with the case of a 'typical' patient as a framework. 


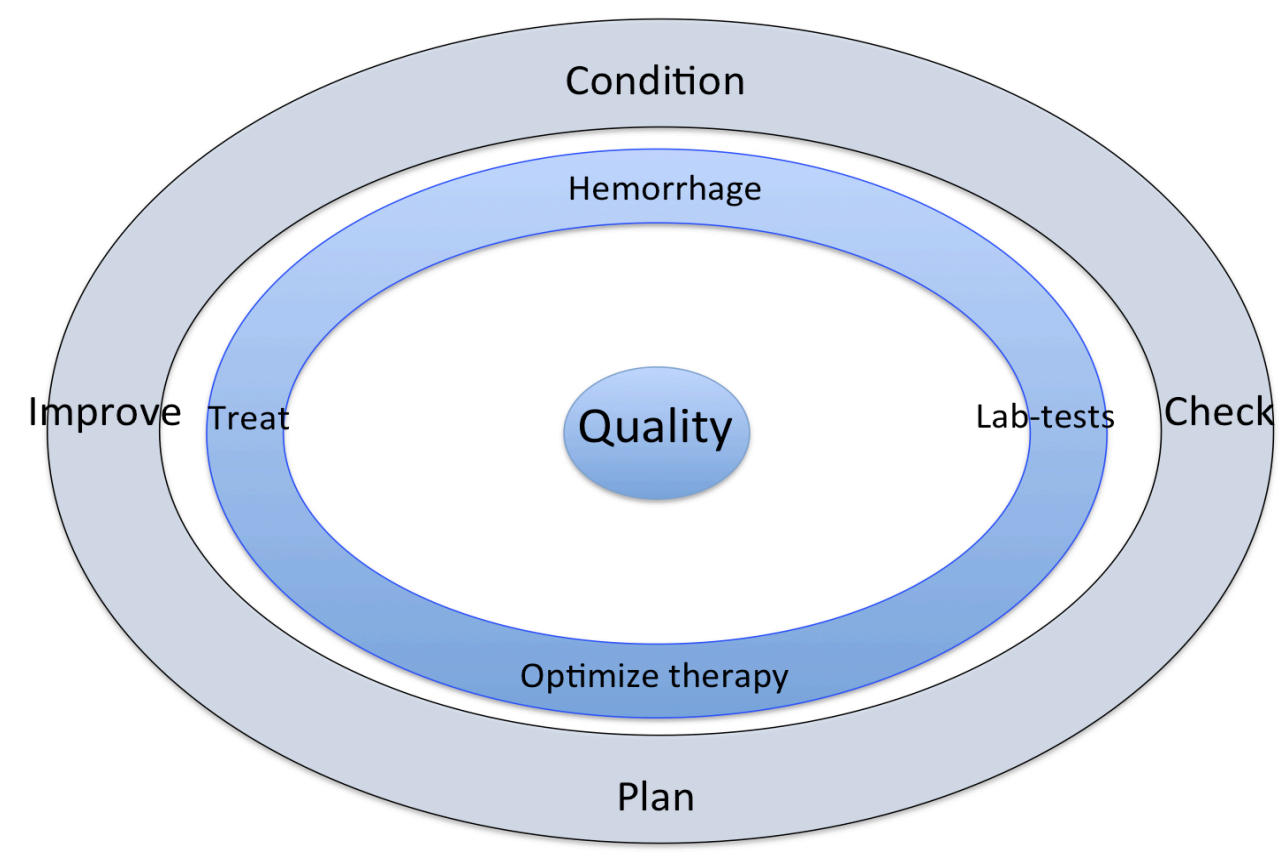

Figure 1: Circle of improvement applied to the management of hemorrhage. As hemorrhage is guided by laboratory tests therapy might become optimized, which leads to better therapy.

Pain! Terrible pain runs through his body peaking in his right leg. He shivers and thinks: "What happened? Why is it so wet?" "Hi, mister, my name is Paul. Can you hear me?" It was Paul's first morning on the ambulance. "You fell with your bike and must have laid about an hour on the side-strip. Can you hear me?" Oh yes, he can, but this pain! He is freezing, although it seems to be warm and bright around him. "Yes", he murmurs, "My name is Dekker. I must have slipped on the wet leaves. "He feels sleepy. "Your right leg is broken. We will bring you to the emergency room in a nearby local hospital." Paul thinks about his course on trauma care and checks all points. Yes, his patient looses consciousness, is cold and has marginally blood pressure with a high irregular, heart rate. Shock! These are the first signs of shock he remembers. He has to avoid the lethal triad of trauma: undercooling, hemodilution and acidosis.

The combination of hemodilution, acidosis and hypothermia is commonly known as the lethal triad of trauma [14]. Hemodilution is present in $10-50 \%$ of all trauma patients and depends on international differences between fluid management in treatment of massive hemorrhage $[15,16]$. When patients are infused with larger amounts of fluids hemorrhage becomes enhanced and mortality increases [17]. In vitro, a sample dilution of $30-40 \%$ affects coagulation negatively by lowering the results of viscoelastic measurements $[18,19]$. In chapter $\mathbf{6 a}$, we showed in patients suffering from perioperative hemorrhage that impairment of hemostasis may be seen in thrombin generation (measured with the CAT assay), in fibrin clot formation 
(measured by thromboelastography) or in both processes. This could be explained by sub-threshold levels of either vitamin K-dependent coagulation factors or fibrinogen, respectively. As indicated in the literature, also other processes and factors can play a role, including the anticoagulant protein $\mathrm{C}$ pathway and the process of fibrinolysis $[20,21]$.

Acidosis as a result of tissue hypoperfusion and shock is defined as a $\mathrm{pH}$ of $<7.2$ with a base excess below $6 \mathrm{mEqu} / \mathrm{L}$ and lactate level above $2.2 \mathrm{mmol} / \mathrm{L}$ and is an important contributor to coagulopathy, because most of the coagulation enzymes work optimal at a pH of 8-8.5 [22]. Martini and co-workers showed in an animal model that the propagation phase of thrombin generation is inhibited by $50 \%$ at a low $\mathrm{pH}$. In that situation the amount of fibrinogen is decreased by $35 \%$ and the platelet count is reduced by $50 \%$ [23]. However acidosis is not only a result of shock, but also of the ensuing massive transfusion, because older RBC's have a BE of $-50 \mathrm{mmol} / \mathrm{L}$ [24]. The importance of normo-acedemia is stressed by the dysfunction of factor VIla, below a pH of 7.2, which has to be reached before this agent works optimally [25]. Finally, hypothermia defined as body temperature below $35^{\circ} \mathrm{C}$, is an independent risk factor for mortality $[26,27]$. About $10-50 \%$ of all trauma patients arrive with hypothermia in the hospital or further decrease in temperature during the first 24 hours after trauma. Although the amount of blood loss increases with $16 \%$ per $1{ }^{\circ} \mathrm{C}$ in laboratory studies, mild hypothermia is mainly associated with platelet dysfunction. Blood temperature below $33^{\circ} \mathrm{C}$ may lead to enzyme dysfunction of the coagulation cascade [28, 29]. Moreover, in presence of hypothermia, acidemia worsens coagulation more than each single factor [30,31]. Current guidelines recommend preventing the lethal triad by striving after normalization of temperature and acidbase status while restricting fluid infusion and treating coagulopathy aggressively [32]. Although we know that hypothermia is associated with coagulopathy we do not know the precise effects of the depth of hypothermia and speed of cooling on coagulation dynamics. Further is it unknown, how the coagulation system behaves when the patient is re-warmed. In future research these questions together with exact mechanism should to be addressed. 
"So warm the patient up!" Paul thinks. Shall he infuse now a large amount of fluids? Paul is uncertain. He knows that shock will increase the risk of organ damage and induce acidosis, but hemodilution probably will increase the hemorrhage out of Mr. Dekker's open femur-fracture. A start into the bloody vicious circle of coagulopathy! "Accept a systolic blood pressure of about $90 \mathrm{mmHg}$ as permissive hypotension" he remembers the lectures. Paul prepares a crystalloid infusion, but would it not be better to start transfusion of blood products now to avoid hemodilution?

As hemodilution aggravates hemorrhage, its treatment should focus on avoiding further dilution. Early transfusion of blood products might support hemostasis and of course surgical control of bleeding. This concerns transfusion with packed red cells, fresh frozen plasma and platelet concentrates, because blood products need special storage conditions, ABO-cross-matching and safety measurements, which are logistically difficult to reach in preclinical setting. Although it is possible to transfuse blood products without cross-matching (e.g. without regard to ABO compatibility) the use of large amounts of $O$ negative red blood cells is associated with higher mortality $[33,34]$. Treatment with plasma as a source of coagulation factors in a preclinical setting is not applicable due to the fact that plasma's maximal storage time is one year when frozen at $-18^{\circ} \mathrm{C}$ and about 6 days at $4^{\circ} \mathrm{C}$ [35]. A possible alternative might be frozen-dried plasma, which can be stored at room temperature with a shelf life of one year without need for ABO-compatibility and a reliable safety-profile [36].

Moreover, FFP contains approximately $0,6 \mathrm{~g} / \mathrm{L}$ fibrinogen and so plasma transfusion may result in even lowering of the patient's fibrinogen level [37]. A combination of FFP with fibrinogen might diminish the dilutional effects of FFP transfusion without fibrinogen. In this thesis, we also considered the use of combination of FFP with fibrinogen, which may diminish the dilutional effects of FFP transfusion. As we showed in chapter $\mathbf{6 b}$, this approach is as effective as conventional treatment, at least in the clinical setting for patients undergoing major surgery.

The use of coagulation factor concentrates might be an alternative. In animal models of massive bleeding, several authors have demonstrated blood saving effects of infusion of both fibrinogen and prothrombin complex concentrate [38, 39]. Furthermore Schöchl and co-workers showed in a retrospective study in trauma patients the beneficial effects of their use in clinical setting (fibrinogen and prothrombin complex concentrate) [40]. In future research one could include the preclinical period. Here researchers should investigate the optimal product choice or a combination of products and try to develop treatment strategies. 
Maybe an alternative to blood products might be coagulation factor concentrates. As Paul remembers, fibrinogen is the first factor, which drops below a critical level during hemorrhage. Coagulation factor concentrates might be easier to transport and there is no need to match them to the patient's blood group. Could early infusion of coagulation factors improve outcome? Since there is no consensus in the clinical settings, how can Paul decide what to do or not to do?

Other authors did also show that the transfusion of coagulation factor concentrates improves the patient outcome in severe trauma and bleeding after cardiac surgery [40-42]. Given that fibrinogen is the first factor, which decreases below critical limits during bleeding, the current lower limit for treatment of $1 \mathrm{~g} / \mathrm{L}$ is under discussion [43, 44]. Recent guidelines recommend a higher level of $>1.5 \mathrm{~g} / \mathrm{L}$ in trauma [32]. On the other hand, in obstetrical hemorrhage even this level may still be too low, as described in chapter $\mathbf{5 a}$. In this situation, a pre-interventional fibrinogen level of 3.4 $\mathrm{g} / \mathrm{L}$ is a predictor for massive transfusion, but it is unclear whether this level should be a therapeutic aim [45]. Fibrinogen infusion may also compensate for a low platelet count [46], but dosing studies have not been carried out here.

A novel approach for the optimization of transfusion therapy upon bleeding is the so-called goal-directed coagulation therapy, advocating the use of point-of-care tests like thromboelastography to guide therapy [47]. The main advantage is the shorter turn-around times to obtain test results, compared to conventional clotting time measurements (aPTT, PT), which take 30-60 minutes [48, 49]. Although pointof-care tests like ROTEM are rapid and easy to use, they are preferably performed by trained personnel, which is present in the hospital laboratory. It would be good to use them together with hemoglobin measurements in a preclinical setting comparable to POC blood glucose measurements. The sensitivity of this equipment to vibration prohibits its use in the ambulance or helicopter. In the absence of measurements, in the acute preclinical situation, the effect of transfusion of an empirical fibrinogen dose is an educated guess, based on a retrospective clinical analysis that a median dose of $2 \mathrm{~g}$ fibrinogen concentrate is able to reduce blood loss [50]. Whether there is a place for robust variants of the ROTEM and whole-blood thrombin generation assays as preclinical point-of-care devices still need to be studied in future investigations. 
However, wouldn't it be better to measure some blood parameters in the ambulance? How about a hemoglobin level, platelet count? Would a fibrinogen level help predicting blood loss as it does in obstetrical bleedings? During his training there was the cardiac-anesthesiologist who told them that fibrinogen levels might predict the amount of blood loss. Oh, yes a ROTEM might help. While Paul searches for a venous line he doubts if he should draw a blood sample. What if he uses a wrong technique? Would it influence the results? No, he was told this by one of the lecturers during the trauma course. "It will not make a difference if blood samples are drawn from an artery or a vein" he recalls this guy's presentation.

In various chapters of this thesis, we have described the importance of rapid, preferably point-of-care, testing of blood in patients with risk of bleeding before, during and after surgery. For complete understanding of the hemostatic activity, we have argued that the testing is needed of platelets, the coagulation system (including von Willebrand factor), and the formation of a fibrin clot. Most hospital laboratories use highly standardized operating procedures and internal and external quality control measurements for each of these measurements to avoid inaccuracy of results, which lead to incorrect diagnosis and therapy [51, 52]. However, as indicated in chapter 3 , blood testing does not start in the laboratory. Pre-analytical variables of the quality of the blood sample can also influence the test outcome. For instance, it has been shown that the choice of needle bore or the prolonged use of a tourniquet can affect the results of various hematological parameters [51]. Also the choice of collecting tubes (with or without vacuum) and inadequate filling of the tubes is important [53].

The current view is that counting the number of platelets in blood is not enough to assess the in vivo function of circulating platelets [54]. Therefore we examined the question whether measurement on the mean platelet volume (MPV) can be used as a surrogate parameter for platelet function during bleeding [55]. As described in chapter $\mathbf{2 a}$, the four techniques to measure MPV (manual, impedance or optical light scatter counting, and immunological flow cytometry) each give different results for methodological reasons. Also given other pitfalls like the time to MPV measurement, because of platelet swelling, we conclude in chapter $\mathbf{2} \mathbf{b}$ that methods of MPV assessment do not seem to be a reliable parameter of platelet activity in case of massive hemorrhage.

As reported in the recent literature, measurement of platelet function with modern point-of-care devices may provide important information to predict the need for 
perioperative transfusion of platelet concentrates [56-58]. Although some authors have stated that the blood sampling method may influence thromboelastography results [59-62], there is only limited evidence for effects of the sample site (arterial or venous) or sample method on platelet function analysis. In chapter 3 we have shown with both impedance aggregometry and light transmission aggregometry, there is no effect of the blood sampling method or site (arterial, venous or peripheral venous) on platelet aggregability in vitro. Unfortunately, aggregometry cannot be used for patients with a platelet count below $100 \times 10^{9} / \mathrm{L}$, which is not uncommon in bleeding patients. In order to optimize the indication for platelet transfusion it would be important to develop methods to easy assess platelet function in this setting. As a first step a flow cytometry based method has recently been published to assess platelet function in patients suffering from Glanzmann thrombasthenia. However, this is not applicable in clinical practice [63].

"Crash-2"-in this study trauma patients received tranexamic acid after trauma and blood loss. This should be applied within one to three hours post injury; Paul thinks and immediately gives the first dose to Mr. Dekker.

A simple measure to minimize blood loss might be infusion of tranexamic acid, an antifibrinolitic drug, as early as possible. This has been shown recently in a large trial. Although the mortality rate due to bleeding decreased the amount of blood transfusion in both groups was comparable $(50.4 \%$ versus $51.3 \%$ blood transfusion tranexamic acid versus placebo) [64]. However the low costs and few side effects of the drug allow recommending its use in general.

\footnotetext{
"Call the trauma team! This patient is bleeding and going into shock" Paul calls at the entrance of the emergency room in the local hospital. Instantly, Dr. Smits, the emergency physician, runs to Paul and directs him and his patient to the shock room. "This is Mr. Dekker a 56 year old male, who fell with his bike on his right tight. At arrival he was unconscious, cold and had a low blood pressure. He has an open fracture of this tight and is bleeding heavily. I did not see other obvious lesions. After some minutes in the ambulance he woke up and moved all his extremities." At once Mrs. Jansen, the emergency nurse, calls the rest of the trauma team and Dr. Smits activates the massive transfusion protocol. "I put the ECG on", Mrs. Jansen says, "Then I will take a blood sample for instant analysis, blood typing and cross matching sending by pneumatic system to the laboratory. It makes it really fast".
} 
Recent literature strongly recommends the use of special intervention protocols for cases of massive hemorrhage [65, 66]. When a patient loses blood, validated laboratory tests are required for decision-making, but these should be done as fast as possible. The standard coagulation tests (aPTT, PT, INR) take 30-60 minutes after being ordered [48, 49]. This is highly disadvantageous in a dynamic situation like in massive hemorrhage. Furthermore, these standard tests were never developed for predicting bleeding or guiding treatment of hemorrhage. Moreover, they are insensitive to hemodilution, and fail to identify patients with acute or occult bleeding $[67,68]$. So they are not very helpful for the clinician during treatment.

Whole-blood coagulation tests may provide a 'rapid' alternative. As described in chapter 1, whole-blood thromboelastography has been re-discovered in the last decade as fast methods (ROTEM or TEG) to measure the capacity of elastic fibrin clot formation in blood. Already in 1985 Kang and co-workers demonstrated a decrease in blood loss during liver transplantation by implementation of whole blood thromboelastography as a diagnostic tool [69]. About 14 years later Shore-Lesserson created and evaluated a treatment algorithm for cardiac surgical patients based on TEG guidance. The authors were able to reduce both blood loss and the use of blood products [70]. Since that time many authors proofed the effectiveness of these tests in a bedside setting in the operating theatre in different populations [71-75]. However, throughout the history of the viscoelastic tests there are concerns about the reliability and possible lack of standardization of thromboelastometry [76-80]. One way to increase the quality and reliability of the tests, could be taking them back to a laboratory in the (time-)vicinity of the massively bleeding patient, where dedicated people -laboratory technicians- perform them. For this reason we studied whether a pneumatic tube transport to the laboratory maintains or even improves reliability and quality of the measurements without unwanted time delay (Chapter 4a). We demonstrated the feasibility of a pneumatic tube transport for ROTEM analysis and were able to implement the device in the hematological laboratory without time delay. By installing an on-line remote viewer, the caregivers can easily follow the ROTEM analysis while being next to his patient. However, it is necessary to put the ROTEM test results into an algorithm in order to improve patient care and to save blood products. As other authors recently reported this may lead to a reduction in costs as well [72]. For future research it will be important to investigate if the algorithm can be 
improved by adding other tests (e.g. whole blood thrombin generation [81] or a platelet function test applicable in thrombocytopenia).

\begin{abstract}
While Dr. Smits does the quick-scan of Mr. Dekker's belly, the anesthesiologist, the trauma surgeon, the intensivist and the neurologist arrive. Now there is planning and action. The team places two more large bore venous lines and transfuses the early arrival package consisting of 4 units red packed cells, 4 units fresh frozen plasma and 1 unit platelet concentrates, which had just arrived via the pneumatic system. "This decreases the time to transfuse blood products enormous" says the anesthesiologist. "I remember a case when a patient bled to dead due to the late arrival of blood products, but fortunately it has been shown that pneumatic system transport saves time and does no harm to the product." Now, Mr. Dekker gets intubated quickly to secure his airway. Then the emergency team transports him to the computer tomography for a full body scan-including the brain. "Luckily, only an open femur fracture right side and no other lesions." says the trauma surgeon. "But he is still bleeding!"
\end{abstract}

An alternative strategy to stop perioperative bleeding, propagated by Johansson and co-workers, is the use of a so-called early transfusion package, which contains 5 units of packed red blood cells, 5 units of fresh frozen plasma and 2 units of platelet concentrate. This strategy appeared to improve whole-blood thromboelastography parameters and was clinically successful [82, 83]. Fast transport of blood products by pneumatic systems might speed up transfusion and improve hemostasis faster. However transfusion products, especially platelets concentrates, should be of the highest quality possible. While the quality of platelet concentrates is affected by storage and irradiation [84, 85], it is not known whether it is affected by transport via a dedicated pneumatic transport system. As we describe in chapter $\mathbf{4 b}$, platelet concentrates can be sent to the place of action via a pneumatic tube system without compromising the platelet function in vitro.

While Mr. Dekker returns to the emergency room his situation deteriorates as he keeps bleeding. "Should we try a tourniquet?", the young surgeon suggests. "No, we will fix the fracture in the operation theatre." answers Dr Smits. "Here is the current ROTEM analysis. It shows a shortage of fibrinogen and some degree of fibrinolysis, although one dose of tranexamic acid already was given in the ambulance. Shall we repeat or give something else?" Mrs. Jansen asks. Dr Smits decides to give a combination of 4 units FFP and 4 grams of fibrinogen concentrate. "We will also repeat tranexamic acid-2 grams and order 4 units of red packed cells after receiving the latest results from the lab," he says, "because it is not known what the best ratio of FFP to fibrinogen is. After transfusion we will repeat another ROTEM test, a whole blood TG and a test on the multiplate. It is great that, the multiplate could be standardized for low platelet counts. It really is nasty not to know what the 
contribution of the platelets to the coagulation will be. I hope we will get some idea of the drugs the patient uses as he told Paul." Otherwise we might need some activated factor VIla or even some factor XIII?

Our data indicate that the stopping of bleeding is in many cases accompanied by an improved capacity of the blood of fibrin clot formation as well as thrombin generation (chapter 6b). Still in some cases this will not be successful. Here one may think about a deficiency of other coagulation factors, platelet dysfunction and endothelial dysfunction. Most guidelines recommend in this situation the use of rFVIla as a last resort [32]. This may solve the problem, because rFVIla boosts the coagulation extremely. Unfortunately the risk of adverse events (pulmonary embolism, vascular occlusion) increases also [86]. Currently, there are reports on factor XIII infusion in treatment of bleeding, because it contributes to clot firmness. However, the influence of factor XIII cannot be tested quickly by our current coagulation tests. Its clinical effects in massively bleeding patients are not very well investigated. Finally, none of the available coagulation tests is able to quantify the contribution of the endothelium to the hemostasis.

In the operation theatre everything is prepared for the patient. As Mr. Dekker arrives he is covered with warming blankets and fluid warmers are connected to the infusion systems. "May I give the platelet concentrates warm?" the anesthetic nurse asks the anesthesiologist, who answers: "Yes, you even may give them with some pressure as recently was shown". Then surgery starts. Some hours later the patient becomes stable. After giving the tailored hemostatic therapy, which includes transfusion at a ratio for FFP-RBC and PC completed with coagulation factor therapy until the coagulation works well. Then Mr. Dekker arrives on the intensive care unit.

Since the situation is stabilized the young assistant doctor asks the intensivist if he should prescribe some prophylactic LMWH now or better wait until it is sure that the bleeding stopped. He read about an increased risk of thromboembolism after massive transfusion and wonders if he should order some tests to weight the risk of bleeding against the risk of thrombosis. "I would recommend a ROTEM analysis together with a complete blood count", the intensivist replies, "We may add a whole blood thrombin generation to detect hypercoagulability very early." he answers.

The present thesis demonstrates how a circle of improvement (Figure 2) by the means of an exemplary case - a patient with massive bleeding - might be optimized using modern diagnostic tools (chapter 2, 3, 4a), accelerated blood product delivery by pneumatic systems (chapter $\mathbf{4 b}$ ) and imbedding the treatment into algorithms 
(chapter 5a). Finally, we show in chapter 6 how treatment might be optimized according to the results of point of care measurements. Furthermore we point out some future aspects for research, which again might enhance this quality circle.

In the end of our case we indicate that the resolved bleeding problem will result in a new circle of improvement in prevention of thrombosis and thromboembolism, which is important for continuing optimal therapy in intensive care medicine.

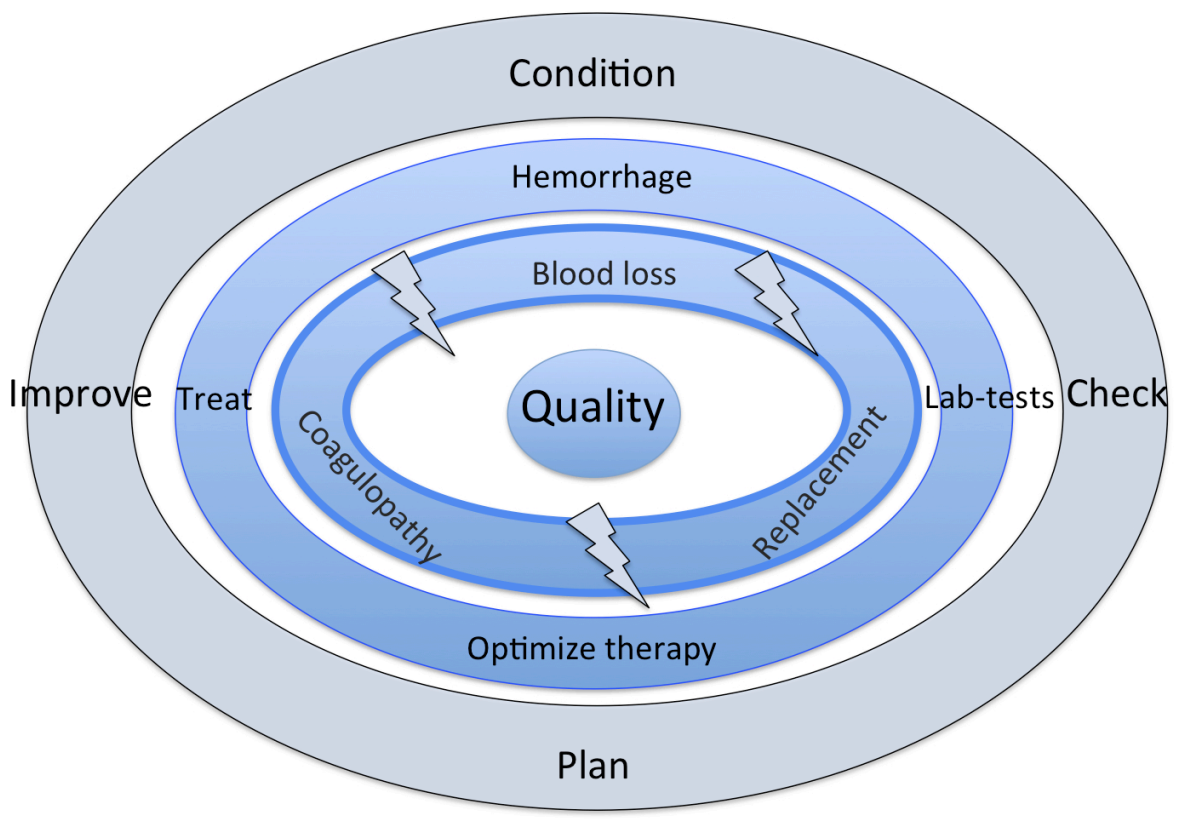

Figure 2: Circle of improvement applied to the bloody vicious circle of coagulopathy. This thesis resulted in improvements at the laboratory side, and at the clinical, therapy side. New developments are needed to be investigated and implemented in this circle as denoted in the text. 


\section{References}

1 Stanworth SJ, Morris TP, Gaarder C, Goslings JC, Maegele M, Cohen MJ, Konig TC, Davenport RA, Pittet JF, Johansson PI, Allard S, Johnson T, Brohi K: Reappraising the concept of massive transfusion in trauma. Crit Care. 2010;14: R239. Moskowitz DM, McCullough JN, Shander A, Klein JJ, Bodian CA, Goldweit RS, Ergin MA: The impact of blood conservation on outcomes in cardiac surgery: is it safe and effective? Ann Thorac Surg. 2010;90: 451-8.

3 Rogers MA, Blumberg N, Saint S, Langa KM, Nallamothu BK: Hospital variation in transfusion and infection after cardiac surgery: a cohort study. BMC medicine. 2009;7: 37.

4 Snyder-Ramos SA, Mohnle P, Weng YS, Bottiger BW, Kulier A, Levin J, Mangano DT: The ongoing variability in blood transfusion practices in cardiac surgery. Transfusion. 2008;48: 1284-99.

Clark SL, Belfort MA, Dildy GA, Herbst MA, Meyers JA, Hankins GD: Maternal death in the 21st century: causes, prevention, and relationship to cesarean delivery. Am J Obstet Gynecol. 2008;199: 36 e1-5; discussion 91-2 e7-11.

6 The world health report 2005 - Make every mother and child count. Geneva: World Health Organisation 2005.

7 So-Osman C, Cicilia J, Brand A, Schipperus M, Berning B, Scherjon S: Triggers and appropriateness of red blood cell transfusions in the postpartum patient--a retrospective audit. Vox Sang. 2010;98: 65-9.

Murphy GJ, Reeves BC, Rogers CA, Rizvi SI, Culliford L, Angelini GD: Increased mortality, postoperative morbidity, and cost after red blood cell transfusion in patients having cardiac surgery. Circulation. 2007;116: 2544-52.

9 Surgenor SD, Kramer RS, Olmstead EM, Ross CS, Sellke FW, Likosky DS, Marrin CA, Helm RE, Jr., Leavitt BJ, Morton JR, Charlesworth DC, Clough RA, Hernandez F, Frumiento C, Benak A, DioData C, O'Connor GT: The association of perioperative red blood cell transfusions and decreased long-term survival after cardiac surgery. Anesth Analg. 2009;108: 1741-6.

Bursi F, Barbieri A, Politi L, Di Girolamo A, Malagoli A, Grimaldi T, Rumolo A, Busani S, Girardis M, Jaffe AS, Modena MG: Perioperative red blood cell transfusion and outcome in stable patients after elective major vascular surgery. Eur J Vasc Endovasc Surg. 2009;37: 311-8.

Glance LG, Dick AW, Mukamel DB, Fleming FJ, Zollo RA, Wissler R, Salloum R, Meredith UW, Osler TM: Association between intraoperative blood transfusion and mortality and morbidity in patients undergoing noncardiac surgery. Anesthesiology. 2011;114: 283-92. Christensen MC, Krapf S, Kempel A, von Heymann C: Costs of excessive postoperative hemorrhage in cardiac surgery. J Thorac Cardiovasc Surg. 2009;138: 687-93.

Stokes ME, Ye X, Shah M, Mercaldi K, Reynolds MW, Rupnow MF, Hammond J: Impact of bleeding-related complications and/or blood product transfusions on hospital costs in inpatient surgical patients. BMC health services research. 2011;11: 135. Holcomb JB: Damage control resuscitation. J Trauma. 2007;62: S36-7.

15 Maegele M, Lefering R, Paffrath T, Simanski C, Wutzler S, Bouillon B: Changes in transfusion practice in multiple injury between 1993 and 2006: a retrospective analysis on 5389 patients from the German Trauma Registry. Transfus Med. 2009;19: 117-24. Brohi K, Singh J, Heron M, Coats T: Acute traumatic coagulopathy. J Trauma. 2003;54: 112730.

17 Maegele M: Frequency, risk stratification and therapeutic management of acute post-traumatic coagulopathy. Vox Sang. 2009;97: 39-49.

Schols SE, Lance MD, Feijge MA, Damoiseaux J, Marcus MA, Hamulyak K, Ten Cate H, Heemskerk JW, van Pampus EC: Impaired thrombin generation and fibrin clot formation in patients with dilutional coagulopathy during major surgery. Thromb Haemost. 2010;103: 31828.

Weiss G, Lison S, Spannagl M, Heindl B: Expressiveness of global coagulation parameters in dilutional coagulopathy. Br J Anaesth. 2010;105: 429-36.

20 Chesebro BB, Rahn P, Carles M, Esmon CT, Xu J, Brohi K, Frith D, Pittet JF, Cohen MJ: Increase in activated protein $C$ mediates acute traumatic coagulopathy in mice. Shock. 2009;32: 659-65.

21 Cohen MJ, Bir N, Rahn P, Dotson R, Brohi K, Chesebro BB, Mackersie R, Carles M, WienerKronish J, Pittet JF: Protein $\mathrm{C}$ depletion early after trauma increases the risk of ventilatorassociated pneumonia. J Trauma. 2009;67: 1176-81. 
Lier H, Krep H, Schroeder S, Stuber F: Preconditions of hemostasis in trauma: a review. The influence of acidosis, hypocalcemia, anemia, and hypothermia on functional hemostasis in trauma. J Trauma. 2008;65: 951-60.

23 Martini WZ, Dubick MA, Pusateri AE, Park MS, Ryan KL, Holcomb JB: Does bicarbonate correct coagulation function impaired by acidosis in swine? J Trauma. 2006;61: 99-106.

24 Zander R: [Pathophysiology of hypovolemic shock]. Anasthesiol Intensivmed Notfallmed Schmerzther. 2001;36 Suppl 2: S137-9.

25 Martinowitz U, Michaelson M: Guidelines for the use of recombinant activated factor VII ( $\mathrm{rFVIIa)} \mathrm{in} \mathrm{uncontrolled} \mathrm{bleeding:} \mathrm{a} \mathrm{report} \mathrm{by} \mathrm{the} \mathrm{Israeli} \mathrm{Multidisciplinary} \mathrm{rFVIla} \mathrm{Task} \mathrm{Force.} \mathrm{J}$ Throm Haemos 2005;3: 640-8.

26 Reynolds BR, Forsythe RM, Harbrecht BG, Cuschieri J, Minei JP, Maier RV, Moore EE, Billiar $E E$, Peitzman AB, Sperry JL: Hypothermia in massive transfusion: have we been paying enough attention to it? J Trauma Acute Surg. 2012;73: 486-91.

27 Ireland S, Endacott R, Cameron P, Fitzgerald M, Paul E: The incidence and significance of accidental hypothermia in major trauma--a prospective observational study. Resuscitation. 2011;82: 300-6.

28 Rajagopalan S, Mascha E, Na J, Sessler DI: The effects of mild perioperative hypothermia on blood loss and transfusion requirement. Anesthesiol. 2008;108: 71-7.

Wolberg AS, Meng ZH, Monroe DM, 3rd, Hoffman M: A systematic evaluation of the effect of temperature on coagulation enzyme activity and platelet function. J Trauma. 2004;56: 1221-8.

30 Dirkmann D, Hanke AA, Gorlinger K, Peters J: Hypothermia and acidosis synergistically impair coagulation in human whole blood. Anesth Analg. 2008;106: 1627-32.

31 Hess JR, Brohi K, Dutton RP, Hauser CJ, Holcomb JB, Kluger Y, Mackway-Jones K, Parr MJ, Rizoli SB, Yukioka T, Hoyt DB, Bouillon B: The coagulopathy of trauma: a review of mechanisms. J Trauma. 2008;65: 748-54.

32 Rossaint R, Bouillon B, Cerny V, Coats TJ, Duranteau J, Fernandez-Mondejar E, Hunt BJ, Komadina R, Nardi G, Neugebauer E, Ozier Y, Riddez L, Schultz A, Stahel PF, Vincent JL, Spahn DR: Management of bleeding following major trauma: an updated European guideline. Crit Care. 2010;14: R52.

33 Chaiwat O, Lang JD, Vavilala MS, Wang J, MacKenzie EJ, Jurkovich GJ, Rivara FP: Early packed red blood cell transfusion and acute respiratory distress syndrome after trauma. Anesthesiol. 2009;110: 351-60.

34 Ball CG, Salomone JP, Shaz B, Dente CJ, Tallah C, Anderson K, Rozycki GS, Feliciano DV: Uncrossmatched blood transfusions for trauma patients in the emergency department: incidence, outcomes and recommendations. Can J Surg . 2011;54: 111-5.

35 von Heymann C, Keller MK, Spies C, Schuster M, Meinck K, Sander M, Wernecke KD, Kiesewetter $\mathrm{H}$, Pruss A: Activity of clotting factors in fresh-frozen plasma during storage at 4 degrees $C$ over 6 days. Transfusion. 2009;49: 913-20.

36 Shuja F, Finkelstein RA, Fukudome E, Duggan M, Kheirbek T, Hamwi K, Fischer TH, Fikry K, deMoya M, Velmahos GC, Alam HB: Development and testing of low-volume hyperoncotic, hyperosmotic spray-dried plasma for the treatment of trauma-associated coagulopathy. $\mathrm{J}$ Trauma. 2011;70: 664-71.

37 Theusinger OM, Baulig W, Seifert B, Emmert MY, Spahn DR, Asmis LM: Relative concentrations of haemostatic factors and cytokines in solvent/detergent-treated and freshfrozen plasma. Br J Anaesth. 2011;106: 505-11.

38 Dickneite G, Pragst I: Prothrombin complex concentrate vs fresh frozen plasma for reversal of dilutional coagulopathy in a porcine trauma model. $\mathrm{Br} J$ Anaesth. 2009;102: 345-54.

39 Grottke O, Braunschweig T, Spronk HM, Esch S, Rieg AD, van Oerle R, ten Cate H, Fitzner C, Tolba R, Rossaint R: Increasing concentrations of prothrombin complex concentrate induce disseminated intravascular coagulation in a pig model of coagulopathy with blunt liver injury. Blood. 2011;118: 1943-51.

40 Schochl H, Nienaber U, Hofer G, Voelckel W, Jambor C, Scharbert G, Kozek-Langenecker S, Solomon C: Goal-directed coagulation management of major trauma patients using thromboelastometry (ROTEM)-guided administration of fibrinogen concentrate and prothrombin complex concentrate. Crit Care. 2010;14: R55.

41 Solomon C, Pichlmaier U, Schoechl H, Hagl C, Raymondos K, Scheinichen D, Koppert W, Rahe-Meyer $\mathrm{N}$ : Recovery of fibrinogen after administration of fibrinogen concentrate to patients with severe bleeding after cardiopulmonary bypass surgery. $\mathrm{Br} \mathrm{J}$ Anaesth. 2010;104: 555-62. 
Innerhofer P, Westermann I, Tauber H, Breitkopf R, Fries D, Kastenberger T, El Attal R, Strasak A, Mittermayr M: The exclusive use of coagulation factor concentrates enables reversal of coagulopathy and decreases transfusion rates in patients with major blunt trauma. Injury. 2012.

43 Martini WZ: Fibrinogen metabolic responses to trauma. Scand J Trauma Resusc Emerg Med. 2009;17: 2.

44 Fries D, Haas T, Klingler A, Streif W, Klima G, Martini J, Wagner-Berger H, Innerhofer P: Efficacy of fibrinogen and prothrombin complex concentrate used to reverse dilutional coagulopathy--a porcine model. Br J Anaesth. 2006;97: 460-7.

45 Huissoud C, Carrabin N, Audibert F, Levrat A, Massignon D, Berland M, Rudigoz RC: Bedside assessment of fibrinogen level in postpartum haemorrhage by thrombelastometry. $\mathrm{Br} \mathrm{J}$ Obst Gyn. 2009;116: 1097-102.

46 Velik-Salchner C, Haas T, Innerhofer P, Streif W, Nussbaumer W, Klingler A, Klima G, Martinowitz $U$, Fries $D$ : The effect of fibrinogen concentrate on thrombocytopenia. Journal of thrombosis and haemostasis : JTH. 2007;5: 1019-25.

47 Gorlinger K, Dirkmann D, Hanke AA, Kamler M, Kottenberg E, Thielmann M, Jakob H, Peters $\mathrm{J}$ : First-line therapy with coagulation factor concentrates combined with point-of-care coagulation testing is associated with decreased allogeneic blood transfusion in cardiovascular surgery: a retrospective, single-center cohort study. Anesthesiol. 2011;115: 1179-91.

48 Kashuk JL, Moore EE, Sawyer M, Le T, Johnson J, Biffl WL, Cothren CC, Barnett C, Stahel P, Sillman CC, Sauaia A, Banerjee A: Postinjury coagulopathy management: goal directed resuscitation via POC thrombelastography. Ann Surg. 2010;251: 604-14.

49 Kozek-Langenecker SA: Perioperative coagulation monitoring. Best Pract Res Clin Anaesthesiol. 2010;24: 27-40.

50 Thorarinsdottir HR, Sigurbjornsson FT, Hreinsson K, Onundarson PT, Gudbjartsson T, Sigurdsson GH: Effects of fibrinogen concentrate administration during severe hemorrhage. Acta Anaesthesiol Scand. 2010;54: 1077-82.

51 Lippi G, Salvagno GL, Montagnana M, Poli G, Guidi GC: Influence of the needle bore size on platelet count and routine coagulation testing. Blood Coagul Fibrinolysis. 2006;17: 557-61.

52 Mannuci PM, Tripodi A: Mechanisms, markers and management of hypercoagulable states. Haemostasis. 1996;26 Suppl 4: 1-8.

53 Bowen RA, Hortin GL, Csako G, Otanez OH, Remaley AT: Impact of blood collection devices on clinical chemistry assays. Clin Biochem. 2010;43: 4-25.

54 Cauwenberghs S, Feijge MA, Theunissen E, Heemskerk JW, van Pampus EC, Curvers J: Novel methodology for assessment of prophylactic platelet transfusion therapy by measuring increased thrombus formation and thrombin generation. Br J Haematol. 2007;136: 480-90.

55 Martin JF, Trowbridge EA, Salmon G, Plumb J: The biological significance of platelet volume: its relationship to bleeding time, platelet thromboxane B2 production and megakaryocyte nuclear DNA concentration. Thromb Res. 1983;32: 443-60.

56 Reece MJ, Klein AA, Salviz EA, Hastings A, Ashworth A, Freeman C, Luddington RJ, Nair S, Besser MW: Near-patient platelet function testing in patients undergoing coronary artery surgery: a pilot study. Anaesthesia. 2011;66: 97-103.

57 Solomon C, Hartmann J, Osthaus A, Schochl H, Raymondos K, Koppert W, Rahe-Meyer N: Platelet concentrates transfusion in cardiac surgery in relation to preoperative point-of-care assessment of platelet adhesion and aggregation. Platelets. 2010;21: 221-8.

58 Emeklibas N, Kammerer I, Bach J, Sack FU, Hellstern P: Preoperative hemostasis and its association with bleeding and blood component transfusion requirements in cardiopulmonary bypass surgery. Transfusion. 2012.

59 Rubens FD, Labow RS, Waghray G, Robblee J: The importance of sampling site in the measurement of whole-blood platelet flow cytometry. J Cardiothorac Vasc Anesth. 1998;12: 309-13.

60 Manspeizer HE, Imai M, Frumento RJ, Parides MK, Mets B, Bennett-Guerrero E: Arterial and venous Thrombelastograph variables differ during cardiac surgery. Anesth Analg. 2001;93: 277-81.

61 Frumento RJ, Hirsh AL, Parides MK, Bennett-Guerrero E: Differences in arterial and venous thromboelastography parameters: potential roles of shear stress and oxygen content. J Cardiothorac Vasc Anesth. 2002;16: 551-4.

62 Heap MJ, Ridley SA, Hodson K, Martos FJ: Are coagulation studies on blood sampled from arterial lines valid? Anaesthesia. 1997;52: 640-5. 
van de Vijver E, De Cuyper IM, Gerrits AJ, Verhoeven AJ, Seeger K, Gutierrez L, van den Berg TK, Kuijpers TW: Defects in Glanzmann thrombasthenia and LAD-III (LAD-1/v) syndrome: the role of integrin beta1 and beta3 in platelet adhesion to collagen. Blood. 2012;119: 583-6.

64 Shakur H, Roberts I, Bautista R, Caballero J, Coats T, Dewan Y, El-Sayed H, Gogichaishvili T, Gupta S, Herrera J, Hunt B, Iribhogbe P, Izurieta M, Khamis H, Komolafe E, Marrero MA, Mejia-Mantilla J, Miranda J, Morales C, Olaomi O, Olldashi F, Perel P, Peto R, Ramana PV, Ravi RR, Yutthakasemsunt S: Effects of tranexamic acid on death, vascular occlusive events, and blood transfusion in trauma patients with significant haemorrhage (CRASH-2): a randomised, placebo-controlled trial. Lancet. 2010;376: 23-32.

65 Thomas D, Wee M, Clyburn P, Walker I, Brohi K, Collins P, Doughty H, Isaac J, Mahoney PM, Shewry L: Blood transfusion and the anaesthetist: management of massive haemorrhage. Anaesthesia. 2010;65: 1153-61.

66 Nunez TC, Young PP, Holcomb JB, Cotton BA: Creation, implementation, and maturation of a massive transfusion protocol for the exsanguinating trauma patient. J Trauma. 2010;68: 1498505.

67 Eckman MH, Erban JK, Singh SK, Kao GS: Screening for the risk for bleeding or thrombosis. Ann Intern Med. 2003;138: W15-24.

68 Segal JB, Dzik WH: Paucity of studies to support that abnormal coagulation test results predict bleeding in the setting of invasive procedures: an evidence-based review. Transfusion. 2005;45: 1413-25.

69 Kang YG, Martin DJ, Marquez J, Lewis JH, Bontempo FA, Shaw BW, Jr., Starzl TE, Winter PM: Intraoperative changes in blood coagulation and thrombelastographic monitoring in liver transplantation. Anesth Analg. 1985;64: 888-96.

70 Shore-Lesserson L, Manspeizer HE, DePerio M, Francis S, Vela-Cantos F, Ergin MA: Thromboelastography-guided transfusion algorithm reduces transfusions in complex cardiac surgery. Anesth Analg. 1999;88: 312-9.

71 Romlin BS, Wahlander H, Berggren H, Synnergren M, Baghaei F, Nilsson K, Jeppsson A: Intraoperative thromboelastometry is associated with reduced transfusion prevalence in pediatric cardiac surgery. Anesth Analg. 2011;112: 30-6.

72 Weber CF, Gorlinger K, Meininger D, Herrmann E, Bingold T, Moritz A, Cohn LH, Zacharowski K: Point-of-Care Testing: A Prospective, Randomized Clinical Trial of Efficacy in Coagulopathic Cardiac Surgery Patients. Anesthesiol. 2012;117: 531-47.

73 Spalding GJ, Hartrumpf M, Sierig T, Oesberg N, Kirschke CG, Albes JM: [Bedside thrombelastography. Cost reduction in cardiac surgery]. Anaesthesist. 2007;56: 765-71.

74 Rahe-Meyer N, Pichlmaier M, Haverich A, Solomon C, Winterhalter M, Piepenbrock S, Tanaka KA: Bleeding management with fibrinogen concentrate targeting a high-normal plasma fibrinogen level: a pilot study. Br J Anaesth. 2009;102: 785-92.

75 Tanaka KA, Bolliger D, Vadlamudi R, Nimmo A: Rotational Thromboelastometry (ROTEM)Based Coagulation Management in Cardiac Surgery and Major Trauma. J Cardiothorac Vasc Anesth. 2012.

76 Chitlur M, Sorensen B, Rivard GE, Young G, Ingerslev J, Othman M, Nugent D, Kenet G, Escobar M, Lusher J: Standardization of thromboelastography: a report from the TEG-ROTEM working group. Haemophilia. 2011;17: 532-7.

77 Kitchen DP, Kitchen S, Jennings I, Woods T, Walker I: Quality assurance and quality control of thrombelastography and rotational Thromboelastometry: the UK NEQAS for blood coagulation experience. Semin Thromb Hemost. 2010;36: 757-63.

78 Mauch J, Spielmann N, Hartnack S, Madjdpour C, Kutter AP, Bettschart-Wolfensberger R, Weiss M, Haas T: Intrarater and interrater variability of point of care coagulation testing using the ROTEM delta. Blood Coagul Fibrinol. 2011;22: 662-6.

79 Strassner H: Die Entwicklung der Thromboelastographie ans Messverfahren; Med Diss der technischen Universitaet Muenchen. Muenchen, 2002.

80 Holzknecht F: Die Auswertungen des Thromboelastogramms. Basel-New York: Karger, S.; 1962.

81 Ninivaggi M, Apitz-Castro R, Dargaud $\mathrm{Y}$, de Laat B, Hemker HC, Lindhout T: Whole-blood thrombin generation monitored with a calibrated automated thrombogram-based assay. Clin Chem. 2012;58: 1252-9.

82 Johansson PI, Bochsen L, Stensballe J, Secher NH: Transfusion packages for massively bleeding patients: the effect on clot formation and stability as evaluated by Thrombelastograph (TEG). Transfus Apher Sci. 2008;39: 3-8. 
83 Johansson PI, Stensballe J: Effect of Haemostatic Control Resuscitation on mortality in massively bleeding patients: a before and after study. Vox Sang. 2009;96: 111-8.

84 Apelseth TO, Bruserud O, Wentzel-Larsen T, Bakken AM, Bjorsvik S, Hervig T: In vitro evaluation of metabolic changes and residual platelet responsiveness in photochemical treated and gamma-irradiated single-donor platelet concentrates during long-term storage. Transfusion. 2007;47: 653-65.

85 Keuren JF, Cauwenberghs S, Heeremans J, de Kort W, Heemskerk JW, Curvers J: Platelet ADP response deteriorates in synthetic storage media. Transfusion. 2006;46: 204-12.

86 Hsia CC, Chin-Yee IH, McAlister VC: Use of recombinant activated factor VII in patients without hemophilia: a meta-analysis of randomized control trials. Ann Surg. 2008;248: 61-8. 
Summary 
Chapter 1 gives a historical overview of major discoveries in the hemostasis field on the laboratory testing of coagulation and platelet function. In the present thesis we have used three different types of hemostatic screening tests (with plasma or whole blood) to predict hemostatic insufficiencies and to monitor the risk of acquired bleeding. The first category consists of screening tests for plasmatic coagulation kinetics: the prothrombin time, the activated partial thromboplastin time, and kinetic analysis of thrombin generation. Both types of coagulation times were developed in the past during research on coagulation mechanisms. After later standardization, these tests are worldwide used to screen for single or multiple coagulation factor deficiencies, and for monitoring therapeutic anticoagulation with vitamin $K$ antagonists or unfractionated heparin. The first steps towards thrombin generation tests were already set in 1953. Current methods adequately measure the total amount of thrombin generated in coagulating plasma. However, this technique still needs (pre-)analytical standardisation before common use in clinical practice. The second category of screening tests aims to measure the coagulation capacity of whole blood by means of visco-elasticity assessment of fibrin clot formation. Tests in use are the thromboelastography (TEG) and rotational thromboelastometry (ROTEM). The first description of a whole blood visco-elastic test was published in 1948. The method has seen a recent revival, and is currently popular as a fast pointof-care test to monitor bleeding patients. A third relevant group of tests evaluates platelet function analysis in plasma or blood. This includes the platelet function analyzer PFA-100, and multiple electrode impedance aggregometry (MEA). Such rapid tests are important due to the growing number of patients on platelet-inhibiting drugs.

The overall aim of this thesis was to come to a better performance of the circle of quality improvement in bleeding management in the hospital. This quality improvement relies on close collaboration between caregivers and the hospital laboratory, in order to continuously ameliorate the treatment of hemorrhagic patients. To achieve this goal, we investigated how the above mentioned laboratory tests can be used to improve diagnostics in hemostatic control of a given patient, and can help to develop a patient-tailored therapy plan, to stop peri-operative bleeding earlier.

Chapter 2 concentrates on the precise analytical conditions for measurement of the mean platelet volume (MPV). This is relevant since multiple papers consider the MPV as a proxy marker of platelet reactivity and an independent risk factor for 
cardiovascular disease. This presumes a thorough control of the pre-analytical variability of this parameter, and points to a need for good pre-analytical standards. Chapter $\mathbf{2 a}$ consists of a review, which stresses the high variability in literature results describing MPV as a marker of platelet function in cardiovascular disease. It further provides an in-depth survey of the methods for MPV measurement and their drawbacks. Several authors have reported that MPV values are dependent on the type of anticoagulant and the time-to-measurement. Hence, a standardization of laboratory methods for this blood parameter is essential. A well-performing, automated assay is the impedance method, used to measure blood cell counts, which also gives routine information on MPV. A possible approach to standardization of this parameter is proposed.

The study of chapter $\mathbf{2 b}$ aimed to achieve such standardization. Blood from 20 healthy volunteers was collected into tubes containing potassium EDTA or sodium citrate. Platelet count and MPV were determined in the blood samples every half hour. Citrate-anticoagulated samples gave a significantly lower MPV than EDTAanticoagulated samples. The MPV values furthermore increased during two hours in the presence of EDTA, and during 1 hour with citrate. Similar values of MPV under optimal conditions were obtained with blood from a second group of 100 healthy donors to determine reference ranges. These were 7.2-10.8 $\mathrm{fL}$ and 6.1-9.5 $\mathrm{fL}$ for EDTA- and citrate-anticoagulated blood samples, respectively. Accordingly, this pointed to lower MPV values of platelets in citrated whole blood.

In chapter 3 also other pre-analytical factors that might influence platelet function analysis were under investigation. Platelet function measurements in blood samples can be influenced by residual coagulation, but how is not completely understood. We compared how the site of blood collection and the type of blood puncture could affect whole-blood platelet function, as determined by MEA and PFA100. In addition, we determined whether contact activation of the coagulation system was of influence on these measurements in whole blood. Blood sampling from arterial or venous lines or by venipuncture gave similar results for MEA and PFA-100 assays. Contact activation was always detectable, but did not appear to influence the test outcomes. It is concluded that, for platelet function analysis, blood can be collected from venous or arterial lines or by venipuncture.

Chapter $\mathbf{4}$ is devoted to practical questions: how to transport blood samples and blood products efficaciously and without damage through the hospital, 
particularly in relation to point-of-care testing. This was determined by ROTEM, which was used as a whole blood point-of-care test measuring changes in coagulation and in fibrin clot formation. It generates results quickly and is theoretically useful for individualized guided hemostatic therapy. We considered that a centralized use of this method in one location of the hospital may avoid non-expert handling, and therefore investigated the possibility of sending blood samples to this central place through a pneumatic tube system (PTS). The study in chapter 4a aimed to evaluate the influence of PTS transport of blood samples on fibrin clot formation and thrombin generation triggered by tissue factor or contact activation. The results indicated that whole-blood samples can indeed be sent by PTS for valid ROTEM analysis. Transport via PTS did not result in a measurable increase in contact activation. Given that many hospitals have different types of PTS, the present findings may not be valid for all hospitals.

In chapter $\mathbf{4 b}$ the feasibility of platelet concentrate transport in a pneumatic system was under investigation. Again, it was considered that the use of PTS for transport of blood samples in hospitals reduces the workload and turnaround time of test results. PTS may also be used for the transport of blood products for transfusion purposes, but this has not sufficiently been investigated. In this paper, platelet functionality was investigated after single or multiple transports via PTS of platelet concentrates (non-radiated or irradiated), in comparison to storage of the concentrates. Before and after transport, we measured platelet count, platelet metabolic markers, optical aggregation, impedance aggregation, P-selectin (CD62P) expression, and microparticle formation. We found that platelet metabolic markers changed as a result of sample storage, but not by using PTS. Platelet function markers were for the major part not different in concentrates sent by PTS. This work shows that PTS transport of platelet concentrates from the laboratory to the operation theatre is possible without detectable loss in platelet function.

Chapter 5a gives a comprehensive description of current treatment options for obstetric patients presenting with peripartal bleeding. A special focus of this review was to determine the potential of treatment guided by point-of-care devices (rotational thromboelastometry). Worldwide, postpartum hemorrhage is the leading cause of maternal morbidity and mortality. Commonly, however, medical and transfusion therapy is not based on the actual coagulant state, because conventional laboratory test results are not available within $45-60$ minutes. Literature analysis 
indicated a good correlation between thromboelastometric assays and classic coagulation times. Dilution of the blood, particularly after transfusion with colloid fluids, appears to influence the coagulation tests. Fibrinogen in plasma seems to play a major role in the course of postpartum hemorrhage, and its lowering can be an early predictor of the severity of postpartum hemorrhage. The ROTEM value (measured as FIBTEM, assessing fibrin polymerization) appears to decline even sooner than the fibrinogen level and, hence, might be useful for early guidance of interventions. Data on TEM and ROTEM measurements in blood from pregnant women are limited, particularly during the peripartum period and in postpartum hemorrhage. So more research in this field is needed.

Chapter $\mathbf{5 b}$ gives a clinical example of the potential advantages in predicting the bleeding risk in an obstetric patient suffering from HELLP syndrome by way of adequate platelet function analysis. We present a case of a primigravid woman developing HELLP syndrome at 29 weeks and 6 days. Platelet function was assessed by MEA and PFA-100. In addition, platelet count and MPV were monitored over an eleven days period. The results suggested that the outcome parameters of MPV and PFA-100 are better predictors for platelet function than platelet count. Implementation of these tools might improve decision-making and treatment in patients during delivery.

In chapter $6 \mathbf{a}$ the effects of hemorrhage and hemodilution on thrombin generation and fibrin clot formation assessed by thromboelastometry in three different patient groups: patients on vitamin $K$ antagonists $(n=10)$; and cardiopulmonary bypass patients with no major bleeding $(n=30)$ and with risk of major bleeding $(n=58)$. Treatment with vitamin $K$ antagonists led to a progressive reduction in thrombin generation, but not in fibrin clot formation. In patients with no major bleeding, plasma factor levels post-surgery were $53-60 \%$ of normal. This was accompanied by a moderate reduction in both hemostatic processes. In plasmas from (bleeding) patients undergoing major surgery, factor levels were $38-41 \%$ of normal, and these levels increased after plasma transfusion. Taking preset threshold values for normal thrombin generation and fibrin clot formation, at least one process was low in $88-93 \%$ of the patients with (persistent) bleeding, but only in $40-53 \%$ of the patients without bleeding. The processes of thrombin generation and fibrin clot formation were independently reduced in acquired dilutional coagulopathy, while minimal levels of both appeared to be required for adequate hemostasis. 
This leads to chapter $\mathbf{6 b}$ where a treatment strategy (addition of fibrinogen concentrate to the transfusion products) was prospectively studied in a population suffering from massive hemorrhage due to major elective surgery. It could be shown that 2 grams of fibrinogen concentrate will result in better coagulation parameters when assessed by thrombin generation measurement and ROTEM analysis. However, this does not translate into clinical improvement of hemostasis. The results suggest that the amount of fibrinogen has to be adjusted to show also clinical enhancement of the coagulation.

In chapter $\mathbf{7}$ the results from all of these investigations are put into the context of optimized patient care as a circle of improvement in bleeding management, with a focus on future developments. 


\section{Samenvatting}


Hoofdstuk 1 geeft een historisch overzicht van bijzondere ontwikkelingen op het gebied van hemostase-testen. De focus ligt op drie soorten van zogenaamde screenings hemostase-testen in zowel plasma als volbloed, die een bloedingsrisico kunen voorspellen en eventuele verworven stollingsstoornissen kunnen monitoren. De eerste categorie bevat screenings testen, die de kinetiek van plasmatische stolling onderzoeken: de protrombinetijd (PT), de geactiveerde partiële trombinetijd (aPTT) en kinetische analyse van de trombinegeneratie. In het verleden werden de PT en aPTT ontwikkeld tijdens onderzoek naar stollingsmechanismen. Later zijn deze testen wereldwijd gestandaardiseerd voor het opsporen van stollingsfactor deficiënties en voor het monitoren van therapeutische antistolling met vitamine-K antagonisten of ongefractioneerd heparine. De eerste stappen naar een trombinegeneratietest werden reeds in 1953 gezet. De huidige methoden, ontwikkeld in Maastricht, meten adequaat de totale hoeveelheid van trombinegeneratie in plasma. Voordat deze techniek breed in de dagelijkse klinische praktijk kan worden ingezet, dient nog (pre)analytische standaardisatie gedaan te worden. De tweede categorie van screenings-testen richt zich op het meten van de stollingscapaciteit in volbloed door middel van viscoelasticiteitsmeting bij de vorming van een fibrinestolsel. Veel gebruikte viscoelasticiteitstesten zijn tromboelastastografie (TEG) en rotatietromboelastometrie (ROTEM). De eerste viscoelastische volbloedtesten zijn in 1948 ontwikkeld. Recentelijk wordt deze methode weer veel gebruikt, en staat bekend als dé point-of-care-test voor snelle diagnose van bloedingsproblemen. De derde relevante groep testen analyseert de plaatjesfunctie in plasma of volbloed. Dit betreft de platelet function analyser-100 (PFA-100) en de multiple electrode impedance aggregometry (MEA). Deze snel uit te voeren testen zijn belangrijk, omdat steeds meer patiënten in de perioperatieve fase plaatjesremmers gebruiken.

Het algemeen oogmerk van dit proefschrift is te komen tot een beter functionerende kwaliteitscirkel bij het management van bloedingen in het ziekenhuis. Deze kwaliteitsverbetering behelst een nauwe samenwerking tussen zorgverleners en het ziekenhuislaboratorium om de behandeling van bloedingen continu te verbeteren. Wij onderzochten hoe door middel van geschikte laboratoriumtesten de diagnose van een gegeven patiënt verbeterd kan worden, voor het stelpen van bloedingen. Daarnaast onderzochten wij ook hoe een patiëntgeoriënteerd therapieplan kan worden opgesteld teneinde een bloeding vroegtijdig te stoppen. 
Hoofdstuk 2 beschrijft de precieze analytische condities bij bepaling van het mean platelet volume (MPV). Dit is belangrijk, omdat in verschillende artikelen het MPV als biomarker voor reactiviteit van bloedplaatjes - en zo als onafhankelijke risicofactor voor hart- en vaatziekten - beschrijven. Dit vereist een betrouwbare controle van de pre-analytische factoren van de MPV-meting en vraagt om een degelijke standaardisatie.

Het overzicht in hoofdstuk 2a benadrukt de hoge variabiliteit binnen de literatuur, welke MPV als plaatjesfunctie marker in hart- en vaatziekten beschrijft. Voorts geeft het een diepgaander inzicht in de methodieken en de voor- en nadelen behorend bij het meten van MPV. De literatuur bericht meermaals dat waarden van het MPV afhankelijk zijn van het gebruikte anticoagulans en van het meetmoment. Daarom is een standaardisatie van de laboratoriummethoden voor deze parameter belangrijk. Een doeltreffende methode is de geautomatiseerde impedantietechniek, die voor de telling van bloedcellen wordt gebruikt. Deze techniek geeft tevens routinematig informatie over het MPV, en kan gestandaardiseerd worden.

In het onderzoek beschreven in hoofdstuk $\mathbf{2 b}$ is getracht tot standaardisatie te komen van de bepaling van MPV. Hiertoe werd bloed van 20 vrijwilligers in kaliumEDTA en in natrium-citraat verzameld en werd het plaatjesaantal en het MPV elk half uur in de monsters gemeten. De citraatmonsters lieten een significant lagere MPV zien dan de EDTA-monsters. Verder bleek de MPV in EDTA-monsters gedurende 2 uren en die in citraatmonsters gedurende 1 uur te stijgen. Rekening houdend met deze resultaten werd in een tweede groep van 100 gezonde bloeddonoren de referentiewaarde bepaald. Deze ligt voor EDTA-monsters tussen 7,2 en 10,8 fl en voor citraatmonsters tussen 6,1 en 9,5 fl, daarmee benadrukkend de lagere MPV voor citraatmonsters.

In hoofdstuk 3 zijn enige pre-analytische factoren onderzocht, welke van invloed op de plaatjesfunctietesten kunnen zijn. Het meten van deze functie in bloedmonsters kan zijn verstoord door restactiviteit van de stolling, maar het precieze mechanisme hiervan was onbekend. Wij vergeleken het effect van de plaats van bloedafname en de manier van bloedprikken op plaatjesfunctiemetingen in volbloed, geanalyseerd met de MEA en de PFA-100. Verder onderzochten wij of contactactivering van het stollingssysteem een rol speelt bij deze metingen. Bloedmonsters afgenomen via een arteriële of veneuze lijn, of door middel van een armprik gaven vergelijkbare resultaten met MEA en PFA-100. Ondanks het feit dat contactactivering altijd 
aanwezig was, kon er geen effect op de plaatjesfunctie worden aangetoond. Hieruit kan geconcludeerd worden dat monsters voor betrouwbare plaatjesfunctie-analyse zowel uit een arteriële als veneuze lijn of via venapunctie uit de arm afgenomen mogen worden.

Hoofdstuk 4 betreft praktische vraagstellingen, zoals op welke wijze bloedmonsters en bloedproducten voor point-of-care-testen efficiënt en zonder beschadiging binnen het ziekenhuis getransporteerd kunnen worden. Dit werd geëvalueerd met behulp van de ROTEM als volbloed-test, waarmee veranderingen in de stolling en de fibrinestolselvorming te meten zijn. Deze test geeft snel resultaten en is in theorie handig ten behoeve van geïndividualiseerde hemostase-therapie. Bij voorkeur wordt een dergelijke test uitgevoerd op een centrale locatie in het ziekenhuis, waar ervaren medisch analisten de metingen verrichten. Daarom onderzochten wij de mogelijkheden om de bloedmonsters met de buizenpost te sturen. De studie in hoofdstuk 4a evalueerde de invloed van buizenposttransport op de kwaliteit van de monsters met betrekking tot fibrinestolselvorming en trombinegeneratie ten gevolg van contactactivatie. Uit de resultaten blijkt dat bloedmonsters welke met behulp van de buizenpost verzonden worden een nauwkeurige ROTEM-analyse toelaten. Verder werd er geen contactactivatie gevonden. Gezien het feit dat het buizenpost systeem per ziekenhuis verschilt zijn onze resultaten wellicht niet naar elk ziekenhuis te vertalen.

In hoofdstuk 4b werd de mogelijkheid onderzocht om plaatjesconcentraten met de buizenpost te verzenden, met als voordeel een sneller transport. In deze studie werd de plaatjesaggregatie na enkele of meerdere transporten van plaatjesconcentraten (bestraald of niet-bestraald) middels de buizenpost bekeken in vergelijking met de opslagtijd van de plaatjes. Voor en na het transport werden plaatjesaantal, metabole markers van plaatjesactiviteit, optische en impedantie-aggregometrie, P-selectine (CD62P) -expressie en microparticle-vorming gemeten. De metabole markers van de plaatjes veranderden ten gevolg van een langere opslagtijd, maar niet door buizenpost transport. De meeste markers voor plaatjesfunctie waren niet gewijzigd in de concentraten welke middels buizenpost getransporteerd waren. Deze studie toont daarmee aan dat transport van plaatjesconcentraten via buizenpost zonder meetbaar functieverlies van de plaatjes mogelijk is.

Hoofdstuk 5a geeft een uitgebreide beschrijving van de huidige behandeling van obstetrische patiënten met een post-partale bloeding. De focus van deze review 
betreft de vraag naar de potentiële voordelen van een point-of-care (ROTEM/TEG) behandelingsstrategie van deze patiëntengroep. Wereldwijd draagt post-partale bloeding aanzienlijk bij aan mortaliteit en morbiditeit. Omdat de conventionele stollingstesten (PT, aPTT) niet binnen 45-60 minuten beschikbaar zijn, is de behandeling van deze bloedingen vaak niet gebaseerd op kennis van de stolling. Uit de literatuur blijkt er een goede correlatie te zijn van tromboelastometrische testen en de conventionele stollingstesten. Bloedverdunning met infuusvloeistoffen, in het bijzonder colloïdale vloeistoffen, blijkt de stollingstesten te beïnvloeden. Bij het optreden van post-partale bloedingen blijkt het fibrinogeengehalte in plasma een grote rol te spelen. Een verlaging van dit gehalte wordt als predictor van de ernst van een bloeding gezien. De ROTEM assay (vooral de FIBTEM test, die de polymerisatie van fibrine meet) zakt eerder dan het gemeten fibrinogeengehalte en kan zo van belang zijn bij het vroegtijdig beslissen tot een interventie. Data van de TEG en de ROTEM testen bij zwangere patiënten, in het bijzonder rondom de bevalling en bij post-partale bloeding zijn schaars. Daarom is er op dit gebied meer onderzoek nodig. Hoofdstuk 5b laat zien aan de hand van een casus van een patiënt met HELLP syndroom, hoe adequate plaatjesfunctietesten de kans op bloedingsproblemen kunnen voorspellen. De casus beschrijft een primigravida die een HELLP syndroom ontwikkelt na 29 weken en 6 dagen. Plaatjesfunctie werd gemeten met behulp van MEA en PFA-100. Bovendien werd het plaatjesaantal en het MPV over een periode van 11 dagen gemonitored. De resultaten suggereren dat zowel de MPV- als de PFA-100-metingen een betere maat zijn voor de bepaling van plaatjesfunctie zijn dan het plaatjesaantal. Een introductie van deze testen zou de besluitvorming en behandeling rondom de bevalling kunnen verbeteren.

In hoofdstuk 6a zijn de gevolgen van bloedverdunning op de trombinegeneratie en fibrinestolselvorming met behulp van tromboelastometrie onderzocht bij drie patiëntengroepen: patiënten onder behandeling van vitamine-K antagonisten $(n=10)$, patiënten die een operatie met cardiopulmonale bypass ondergingen zonder ernstige bloeding $(n=30)$, en patiënten met risico op ernstige bloedingen $(n=58)$. Hierbij toonden de patiënten die behandeld waren met vitamine $\mathrm{K}$ een progressieve daling in trombinegeneratie. Plasma van deze patiënten liet echter geen duidelijke verandering in de fibrinestolselvorming zien. Anderzijds waren in plasma van patiënten zonder ernstige bloedingen de stollingsfactorspiegels gereduceerd (53$60 \%$ ). Dit ging samen met een verminderde functie van beide genoemde 
hemostasetesten. In plasma van patiënten die majeure chirurgie ondergingen en een bloedingsneiging hadden, waren de factorspiegels gedaald tot $38-41 \%$ van normaal, en namen deze na plasmatransfusie toe. Op basis van vastgestelde randwaarden voor een normale trombinegeneratie en fibrinestolselvorming, bleek dat $88-93 \%$ van de patiënten met (aanhoudende) bloedingen lager scoorde in één van beide testen, en slechts $40-53 \%$ van de patiënten zonder bloedingen. De processen van trombinegeneratie en fibrinestolselvorming bleken onafhankelijk gereduceerd bij verworven verdunningscoagulopathieën, terwijl minimale activiteit van beide testen nodig was voor een toereikende hemostase.

Dit leidt naar hoofdstuk $\mathbf{6 b}$, waar een behandelingsstrategie van bloedplasma al dan niet gedeeltelijk vervangen door fibrinogeenconcentraat prospectief bestudeerd werd bij patiënten die ten gevolg van majeure electieve chirurgie massale bloedingen vertonen. Aangetoond werd dat de vervanging door fibrinogeenconcentraat tot betere resultaten leidde bij de trombinegeneratie en ROTEM-analyses. Er bleek echter geen verbetering te zijn in de klinische situatie. De resultaten suggereren dat de hoeveelheid van fibrinogeen verhoogd dient te worden om een klinisch effect van verbeterde hemostase teweeg te brengen.

In hoofdstuk 7 zijn de resultaten van alle onderzoeken binnen de context van een optimale behandelingsstratie van de patiënt geplaatst. Deze is gezien als een cirkel waarin de verbetering van bloedingsmanagement een centraal punt in neemt, dit ook met het oog op toekomstige ontwikkelingen. 
Dankwoord 
Graag zou ik enkele mensen willen bedanken welke direct en indirect hebben bijgedragen aan het tot stand komen van dit proefschrift...

Prof. dr. Marcus, beste Marco. Toen ik in Maastricht als staflid begon had je meteen door dat ik een gedreven enthousiastelingen ben. Snel vond ik mijn plaats in de kliniek, ook al was er op dat moment nog te weinig om klinisch onderzoek te doen. Daarom gingen wij op reis langs diverse mensen, op zoek naar inspiratie en samenwerkingsverbanden. Dit leek in het begin lastig. Zo zijn wij op eigen initiatief gestart met een kleine pilot bij obstetrische patiënten, misschien mede omdat het jouw speerpunt is. Het bracht ons de nodige aandacht van de mensen van de hematologie en biochemie. Zo hadden wij plots een leuke bespreking met Lizzy, Saskia en Johan. Dit was de geboorte van het translationele onderzoek met CARIM op het gebied van de stolling. Sindsdien heb je mij gesteund, geholpen en soms ook afgeremd (omdat je weet dat ik nooit nee zeg). Bedankt voor het vertrouwen in mijn enthousiasme en het geduld met mijn onervarenheid bij onderzoek en schrijven. Dank ook voor onze vele waardevolle gespreken. Ik hoop dat wij samen de nu opgestarte lijn kunnen volgen en meer mensen aan de stolling kunnen laten "snuffelen".

Prof. dr. Heemskerk, beste Johan. Je behoort tot één van de mensen die ik bij de eerste gespreken over stollingsonderzoek heb mogen leren kennen. Voor mij was het een ontmoeting met een andere wereld, die van het basale onderzoek. Je straalde tijdens onze vele gesprekken steeds rust en veel kennis uit. De kennis van de stolling was steeds meer dan het cascadenschema zoals ik de stolling kende. Waarschijnlijk was het voor jou net andersom. Ik ben veel te onrustig en soms wat diffuus. Misschien is het de combinatie van ons beider eigenschappen die ons goed liet samenwerken. Onder jouw leiding leerde ik klinische situaties aan de nauwkeurigheid van het laboratorium te koppelen. Daarom mijn dank voor je inzet, je hulp en je vertrouwen. Ik hoop dan ook dat wij onze samenwerking verder mogen continueren.

Dr. Henskens, beste Yvonne. Je bent dé contactpersoon van het klinisch hematologisch laboratorium. Maar niet alleen dát! Je hebt me geleerd altijd een juiste methodiek te hebben: alle gegevens, van de gebruikte buis tot aan de toegepaste 
concentratie, moet correct worden gerapporteerd. En zo heb je ook quasi 'afgedwongen' dat het bijschrift van figuren een gedetailleerde, vaak lange, tekst moet zijn. Zo te schrijven viel me niet altijd mee, maar je had natuurlijk gelijk. Mijn dank hiervoor. Verder was de klinische implementatie van de ROTEM zonder jouw inzet nooit gelukt. Je gedrevenheid heeft ons een groep laten vormen waarmee wij nu verder door willen gaan. Wij hebben vele projecten in het verschiet, dus nu gaat het pas écht interessant worden.

Dr. Hamulyak, beste Karly. Ik was met stomheid geslagen dat men zo 'makkelijk' een slecht geschreven manuscript (waar ik destijds bij jou mee kwam aanzetten) kan omvormen tot een leesbaar artikel. Je liet mij zien hoe je dat deed en inderdaad, het heeft geholpen! Steeds wanneer ik vroeg hoe je dat toch deed glimlachte je en zei “Wanneer jij 30 jaar schrijfervaring hebt, kun jij dat ook!". Dit was voor mij een aanmoediging. Door de lange klinische praktijk en de grote ervaring op het gebied van stolling en hemostase had je altijd goede opmerkingen en suggesties. Hiervoor wil ik je hartelijk danken.

Mijn dank ook aan Dr. van Pampus. Beste Lizzy, mijn dank voor jouw stimulerende vragen en opmerkingen. Je bent een goede link tussen kliniek en het laboratorium. Je weet veel uit de praktijk, maar kunt ook heel goed uitleggen. Het was voor mij steeds een groot plezier met jou over stollingsproblemen (en andere dingen) te discussiëren. Ik hoop dat je binnenkort ook weer actief met de kliniek bezig kunt zijn.

Dr. Schols, beste Saskia. Jij was de pionier. Je had de taak de krachten van de biochemie met die van de kliniek samen te brengen. Onze gezamenlijke studies waren erg leuk, vooral omdat jij steeds erop werd uitgestuurd de monsters op de recovery te gaan ophalen. En toen kwam je met het idee van de fibrinogeenstudie en Geert-Jan Kuiper kon meteen aan de slag. Geert-Jan, je was de eerste WESP die zich bij de anesthesiologie met de stolling bezig hield en dat was zeer zeker niet makkelijk. Dat je nu bezig bent met je eigen proefschrift doet me deugt; ik voel me trots en blij want het onderwerp stolling leeft. 
Marisa Niniviaggi, je was erg bezig met de studie over de FFP's. Vooral het labwerk heb jij gedaan. Jouw inbreng en hulp waren van hoge waarde. Herinner je je dat je in het begin helemaal geen promotie onderzoek wilde doen? Nu hoop ik dat jouw promotieonderzoek lekker loopt en dat we binnen kort jou als doctor mogen vieren. Laat het je gezegd zijn: aan het eind komt op een gegeven moment heel snel in zicht.

Het ICU-team wil ik bedanken voor jullie bereidheid om "alweer" aan een klinisch stollingsonderzoek deel te nemen. Vaak kwam dan de vraag wat wij (de kliniek) er mee moeten. Ik hoop dat dit boekje jullie vragen kan beantwoorden.

De mensen van het laboratorium hematologie: Rick Wetzels en Paul Verhezen. Het was leuk om met jullie samen te werken. In de afgelopen jaren heb ik veel van jullie geleerd. Ook al mocht het ook soms wat lastig zijn, ik zal met nieuwe ideeën en vragen blijven komen! Dit geldt natuurlijk ook voor de mensen van de biochemie: Rene van Oerle, Henri Spronk, Marion Feijge. Zonder jullie stimulerende en kritische vragen en opmerkingen zou ik vaker dingen fout hebben gedaan. Het was altijd leuk bij jullie op een gesprek langs te komen. Hierbij durf ik te stellen dat snoep altijd graag gezien wordt.

Beste collegae van de anesthesiologie en de intensive care. Ik wil jullie bedanken voor het begrip dat ik alweer voor "het scherm" moest gaan zitten om een stuk te schrijven. Er is nu dan eindelijk toch iets uitgekomen...

Meinen Paranimfen Dr. Antoine Simons und Dr. Andreas Grießner:

Lieber Antoine, wir lernten uns während unserer Suche nach Projekten und Arbeitsgruppen kennen. Zu Beginn wirktest Du sehr scheu und zurückhaltend auf mich, aber diesen Eindruck hast Du schnell ins Gegenteil gewandelt! Du bist wirklich alles andere als zurückhaltend (und das ist gut so!). Wenn man mit Dir so durch die Klinik geht, kennst Du jede zweite Person. Beim gemeinsamen Schreiben habe ich viel von Deiner unbeschreiblichen Genauigkeit gelernt. Zum Glück durfte ich Dich auch bei Deiner Promotion begleiten, so konnte ich schon einmal einen Eindruck hiervon gewinnen. Danke, dass Du jetzt mir zur Seite stehst. 
Lieber Andreas, Du bist einer der besten klinischen Anästhesisten, die ich kenne. Du hast mir beigebracht, wie man Anästhesie gut ausübt und dass man die Patientenakten stets komplett kennen muss besonders, wenn man mit Dir auf der Intensivstation Visite macht. Deinen schwarzen Humor habe ich von Beginn an sehr geschätzt und hoffe, dass Du diesen weiterhin behältst! Trotz Deines großen medizinischen Wissens hat es Dich nie an die Universität gezogen. Darum bin ich nun stolz und froh, dass Du bei meiner Promotion dabei bist. Hoffentlich haben wir in Zukunft mehr Zeit um einander zu treffen!

Meine Familie, und hier besonders meiner Liebsten. Andrea, ich weiß, es ist nicht einfach, mit einem Mann zusammen zu leben, der entweder Dienst hat oder, falls er keinen Dienst hat, zu Hause am Rechner sitzt um zu arbeiten, die Aquarien pflegt oder mal wieder beim Sport ist. Vielleicht lasse ich es nicht oft genug erkennen (Blumen sagen auch nicht alles), aber ich weiß sehr wohl, dass Du den Club zusammen hältst und dafür sorgst, dass alles läuft. Hierfür bin ich Dir enorm dankbar. Zum Glück haben wir seit vergangenen Sommer auch noch einen Hund, damit uns auch nach der Promotion bloß nicht langweilig wird. Ich liebe Dich!

Kira und Max, ihr seid die Sonne in meinem (unserem) Leben. Es ist schön zu sehen, wie ihr beinahe täglich größer werdet und das auf allen Ebenen. Macht weiter so! Danke auch für Eure Inspiration - ihr seid echte Künstler :-). 


\section{Curriculum vitae}


Marcus Lancé was born on May $17^{\text {th }}, 1970$ in Rott (community Roetgen), Germany. After graduation at the Städtisches Gymnasium Übach-Palenberg, he went to Berlin to study at the Medical School of the Free University (1991-1995) and the Charite of the Humboldt University (1995-1996). In 1997, he started his residency in anaesthesiology at the Kliniken Maria-Hilf, Department of Anaesthesiology and Surgical Intensive Care, Mönchengladbach, from which he moved in 2001 to the University Hospital of the RWTH Aachen. His medical thesis was approved in 2001 by the Heinrich-Heine University Düsseldorf. In the two following years, he finalized his specialisation in anaesthesiology and his super-specialisation in intensive care. After a two years interim period at the Thorax Centre Rotterdam (the Netherlands), where he gained a high degree of experience in cardiac anaesthesiology, he moved in 2006 to the Departments of Anaesthesiology and Intensive Care Medicine of the $\mathrm{MUMC}^{+}$of Maastricht. Here he works as an anaesthesiologist and intensivist and performed the research described in this PhD thesis under the supervision of Prof. dr. M. Marcus, Prof. dr. J. Heemskerk and Dr. Y. Henskens.

Marcus Lancé is married to Andrea Leon and has 2 marvellous children (Kira and Max). 
Publications 


\section{Articles:}

- Kozek-Langenecker SA, Afshari A, Albaladejo P, Aldecoa Alvarez Santullano C, De Robertis E, Filipescu DC, Fries D, Görlinger K, Haas T, Imberger G, Jacob M, Lancé MD, Llau J, Mallett S, Meier J, Rahe-Meyer N, Samama CM, Smith A, Solomon C, Van der Linden P, Wikkelsø AJ, Wouters P, Wyffels P. Management of severe perioperative bleeding. Guidelines from the European Society of Anaesthesiology. Eur J Anaesth 2013 in press

- Simons AP, Lancé MD, Ganushak YM, Weerwind PW. Hypovolämie während der extrakorporalen Kreislaufunterstützung kann zu Entgasung und arteriellen Mikroblasen führen. Kardiotechnik. 2013; 1:3-8

- Lancé MD, Henskens YMC. Letter to the editor-effect of pneumatic tube transport on rotational thromboelastometry. $\mathrm{Br} J$ Anaesth. 2013 Jan;110(1):142

- Hageman D, Kooman JP, Lancé MD, van Heurn E, Snoeijs GJ. Acute nierschade. Ned Tijdschr Geneeskd. 2012;156:A5057

- de Lange NM, Lancé MD, de Groot R, Beckers EA, Henskens YM, Scheepers HC. Obstetric hemorrhage and coagulation: an update. Thromboelastography, thromboelastometry, and conventional coagulation tests in the diagnosis and prediction of postpartum hemorrhage. Obstet Gynecol Surv. 2012 Sep;67(7):426-35.

- Lancé MD, Sloep M, Henskens YM, Marcus MA. Mean Platelet Volume as a Diagnostic Marker for Cardiovascular Disease: Drawbacks of Preanalytical Conditions and Measuring Techniques. Clin Appl Thromb Hemost. 2012 Nov; 18(6):561-8.

- Lancé MD, Henskens YM, Nelemans P, Theunissen MH, Oerle RV, Spronk HM, Marcus MA. Do blood collection methods influence whole-blood platelet function analysis? Platelets. 2012 May 30. E-pub ahead of print.

- Lancé MD, Kuiper GJ, Sloep M, Spronk HM, van Oerle R, Ten Cate H, Marcus MA, Henskens YM. The effects of pneumatic tube system transport on ROTEM analysis and contact activation assessed by thrombin generation test. Thromb Res. 2012 Sep;130(3):e147-50. 
- Schreiber JU, Lancé MD, Korte MD, Artmann T, Aleksic I, Kranke P. The effect of different lung protective strategies in patients during cardiopulmonary bypass. A semiquantitative meta-analysis of randomized trials. J Cardiathorac Vasc Anesth. 2012 Jun;26(3):448-54

- Henskens YM, Lancé MD, Beckers EAM. Implementatie van de tromboelastografie (ROTEM $\left.{ }^{\circledR}\right)$ in het MUMC+: (On)mogelijkheden van laboratoriumtesten bij bloedverlies en verworven hemostase afwijkingen. NTvB, Vol 5:1. 2012:3-13

- Lancé MD, Marcus MA, van Oerle R, Theunissen HM, Henskens YM. Platelet concentrate transport in pneumatic tube systems - does it work? Vox Sang. 2012: Jul;103(1):79-82

- Lancé MD, Ninivaggi M, Schols SE, Feijge MA, Oehrl SK, Kuiper GJ, Nikiforou M, Marcus MA, Hamulyak K, van Pampus EC, Ten Cate H, Heemskerk JW. Perioperative dilutional coagulopathy treated with fresh frozen plasma and fibrinogen concentrate: a prospective randomized intervention trial. Vox Sang. 2012 Jul;103(1):25-34.

- Alatri A, Armstrong AE, Greinacher A, Koster A, Kozek-Langenecker SA, Lancé MD, Link A, Nielsen JD, Sandset PM, Spanjersberg AJ, Spannagl M. Results of a consensus meeting on the use of argatroban in patients with heparin-induced thrombocytopenia requiring antithrombotic therapy - An European Perspective. Thromb Res. 2012 Apr;129(4):426-33.

- Hamers LA, Linssen CF, Lancé MD, Maessen JG, Weerwind P, Winkens B, Bergmans DC, van Mook WN. Positive cultures from cardiopulmonary bypass: prevalence and relevance regarding postoperative infection. Eur $\mathrm{J}$ Cardiothorac Surg. 2011 Aug;40(2):372-8.

- Kuiper GJ, Lancé MD, Smit-Fun VM, Peeters LL, Marcus MA. Platelet monitoring follow-up in a pregnant patient with HELLP syndrome. Platelets. 2011;22(2):160-3.

- Lancé MD, Henskens YM, Marcus MA, Mean platelet volume needs more standardization. Platelets 2011; 22(3):241

- Simons AP, Reesink KD, Lancé MD, van der Nagel T, van der Veen FH, Weerwind PW, Maessen JG, Reserve-driven flow control for extracorporeal life support: proof of principle. Perfusion. 2010 Jan: 25 (1):25-29 
- Simons AP, Lancé MD, Reesink KD, van der Veen FH, Weerwind PW, Maessen JG, Quantitative assesment of cardiac load-responsiveness during extracorporeal life support: case and rational. J Cardiothorac Surg. 2010 Apr 27:5(1):30

- Simons AP, Lancé MD, Reesink KD, Veen vd FH, Weerwind PW, Pumpenflussregelung mit dynamischer Vorlastmessung für die extrakorporale kardiopulmonale Unterstützung-ein Lösungsansatz? Kardiotechnik. 2010 Mai 19:(2):37-41

- Timmer $\mathrm{V}$, Tijssen FH, Marcus $M$, Lancé $M$ Management van acute postoperatieve pijn na cardiothoracale chirurgie-een overzicht. NtvA 2010: 22; 27-31

- Lancé MD, Oerle van R, Henskens YMC, Marcus MAE, Do we need time adjusted mean platelet volume measurements? Lab-Hematol. 2010;16:28-31

- Simons AP, Reesink KD, Lancé MD, van der Veen FH, de Jong DS, Weerwind PW, Maessen JG, Dynamic filling index: a novel parameter to monitor circulatory filling during minimized extracorporeal bypass. EJCTS, 2009, Aug;36(2):330-4

- Heringlake M, Kindgen-Milles D, Hackmann F, Haake N, Kielstein J, Lancé M, Lufft H, Just S, Trabold B, Scherlitz A, Schirmer U, Schmitt D, Vargas-Hein O, Markewitz A, Pathophysiologie, Prophylaxe und Therapie von Herzchirurgie-assoziierten Nierenfunktionsstörungen. Z Herz- Thorax-, Gefäßchir, 2009, Volume 23 (6), 349-373

- Schols SEM, Lancé MD, Feijge MAH, Damoiseaux J, Marcus MA, Hamulyák $\mathrm{K}$, ten Cate $\mathrm{H}$, Heemskerk JWM, van Pampus ECM, Impaired thrombin generation and fibrin clot formation in patients with dilutional coagulopathy during major surgery. Thromb Haemost. 2010 Feb;103(2):318-28.

- Lancé M, Stessel B, Hamulyák K, Marcus MA, Old and new anticoagulants. Antidotes and measures for acute hemorrhaging and urgent interventions. Anaesthesist. 2010 Jan;59(1):62, 64-8

- Schols SEM, Feijge MAH, Lancé MD, Hamulyak K, ten Cate H, Heemskerk JWM, van Pampus ECM, Effects of plasma dilution on tissue factor-induced thrombin generation and thrombelastography: partly compensating role of platelets, Transfusion. $2008 \mathrm{Nov;48(11):2384-94}$ 
- Klompe L, Lancé M, vd Woerd D, Scohy T, Bogers AJJC, Anaesthesiological and ventilatory precautions during cardiac surgery in Steinert's disease, J Card Surg 22, 2007, 74-75

- Lancé M, Reinges M, Schnoor J, 2003, Klismaphilia and unconscious of unknown origin; Nottfall\&Rettungsmdedizin 2: 112-114

- Luther B, Pillny M, Müller B, Lancé M, Sandmann W, 2001, Is the revascularisation of the pedal arteries worthwhile in diabetic gangrene? VASA suppl 58:34-39

\section{Abstracts:}

- Kuiper, G.J.A.J.M., Emam, L.S.H., Theunissen, H.M.S., Lancé, M.D., Marcus, M.A.E. HEmostasis Registry Of patients in Cardiac Surgery (HEROES-CS): perioperative risk factors for red blood cell transfusion among cardiac surgery patiens. Poster \& abstract. ASA meeting 13-17. October 2012, Washington, USA

- De Fries S., Vincent J., van Mook W.N.K.A., Lancé M.D. Thromboelastometry (ROTEM) guidance for titration of recombinant factor Vlla in acquired hemophilia A, a case report. Poster \& abstract III. NWAC meeting, 24-28. April 2012, Istanbul, Turkey

- Maas S., Lancé M.D., Roekaerts P.M. Cardiac Output measurement: Bioimpedance or Pulse Contour analysis versus Thermodilution. Oral presentation \& abstract, NVIC dagen 2012, 8-10 Feb. Ede, The Netherlands

- Balthasar A.J., Siemonsma M.A., Schalla S., Lancé M.D., van Mook W.N. Stress induced transient cardiomyopathie due to accidental administration of norepinephrine and atropine instead of neostigmine and atropine. Abstract NVIC dagen Feb. 2011.

- Meijers W., Weerwind P.W., Nijs J.F., Vainer J., Lancé M.D., Strauch U., Pragt E., Bouman E., Lochy S., Smets R., Geskes G., Van Garsse L., Kats S., Mochtar B., Roekaerts P.M., Maessen J.G., Donker D.W. Is early initiation of extracorporeal membrane oxygenation critical for rescue support in severe refractory cardiogenic shock? Poster \& Abstract NVVC voorjaarscongres 31. March 2011 
- Lancé M.D., Ninivaggi M., Marcus M. A. E., Hamulyak K., van Pampus E. C. M., ten Cate H., Heemskerk J. W. M. Early transfusion with FFP or FFP plus fibrinogen concentrate in massive hemorrhage: a randomized intervention trial. ESA Amsterdam, 2011, 11-14 ${ }^{\text {th }}$ June.

- De Korte M., Lancé M.D., Kranke P., Schreiber J., Lung management during cardiopulmonary bypass: a systematic review of randomized trials. ESA Amsterdam, 2011, 11-14 ${ }^{\text {th }}$ June \& German cardiothoracic society scientific meeting, November 2011

- Kuiper G.J. A.J.M., Lancé M.D., Sloep M., Spronk H.M.H., Oerle R., Henskens Y.M.C., Marcus M.A.E., Ten Cate H. The influence of pneumatic tube system versus manual transport on activation of coagulation measured with thromboelastometry and thrombin generation. Poster \& abstract, ISTH XXIII, Kyoto, Japan, 23-28.07.2011

- Ninivaggi M., Lancé M.D., Kuiper G.J. A.J.M., Schols S.E.M., Feijge M.A.H., Marcus M.A.E., Ten Cate H., Heemskerk J.W.M., Roles of platelets and coagulation factors in the restoration of impaired hemostasis in patients undergoing cardiac surgery. Poster \& abstract, ISTH XXIII, Kyoto, Japan, 2328.07.2011

- Hughes K., Verheijen L., Feijge M., Kuiper G.J.A.J.M., Lancé M., Heemskerk J.W.M., Effects of desmopressin on hemostatic platelet function in patients undergoing cardiothoracic surgery. Poster \& abstract CARIM-dag, Maastricht 9.11.2011

- Lancé M.D., Theunissen H.M.S., van Oerle R., Henskens Y.M.C., Marcus M.A.E., Is transport of thrombocyte concentrates by a pneumatic tube system feasible? Poster GTH 2010, 23-27.Feb.

- Lancé M.D., Theunissen H.M.S., van Oerle R., Henskens Y.M.C., Marcus M.A.E., The frequency of transport by pneumatic tube systems does not influence in vitro platelet function of fresh or aged thrombocyte concentrates. Poster GTH 2010, 23-27.Feb.

- De Ruiter F.E., Sloep M., Theunissen H.M.S., Marcus M.A.E., Lancé M.D. Cardiac output monitoring: invasive versus non-invasive. Abstract \& Poster, Deutscher Interdisziplinärer Notfallmedizin Kongress, 2010, 25-27 Feb. 
- Ninivaggi M., Lancé M.D., Kuiper G.J., Schols S.E.M., Feijge M.A.H., Marcus M.A.E., ten Cate H., Heemskerk J.W.M., Roles of platelets and coagulation factors in the restoration of impaired hemostasis in patients undergoing cardiothoracic surgery, abstract and poster, Nottingham platelet conference, 15-16-july 2010

- Timmerman M.C., Oerle van R., Henskens Y.M.C., Marcus M.A.E., Lancé M.D., Does pneumatic tube system transport influence TEG and ROTEM thrombelastography/-metry? Poster \& abstract VII researchday NVA, 24th September 2010

- Van Garsse L., van Ommen V., Lancé M., Waltenberger J., Maessen J., Successful start of transapical aortic valve implantation program. Presentation \& abstract, $10^{\text {th }}$ Belgian surgical week, Ostend, Belgium, April/Mai 2009

- Lancé M.D., Fun-Smit V.M., Timmer V.C.M.L., Kroese A., Marcus M.E.A., Impact of platelet function analysis on decision making in preeclampsia and HELLP syndrome. Poster \& abstract ESA, Milan, Italy, Juni 2009

- Lancé M.D., Henskens Y.M.C., Theunissen H.M.S., van Oerle R., Hamulyak K., ten Cate H., Marcus M.A.E., Evaluation of different blood drawing techniques on the results of platelet function tests. Poster \& abstract XXII Congress of the International Society on Thrombosis and Haemostasis, Boston, USA, July 11-16

- Schols S., Lancé M., Feijge M., Damoiseaux J., Marcus M., Hamulyák K., ten Cate H., Heemskerk J. and van Pampus E., Impaired thrombin generation and fibrin clot formation in patients with dilutional coagulopathy in major surgery. Poster \& abstract XXII Congress of the International Society on Thrombosis and Haemostasis, Boston, USA, July 11-16

- Lohuis T.A.J., Tanate N., Hilgers N.A.H., Theunissen H.M.S., Marcus M.A.E., Lancé M. D., Cardiac Output monitoring in cardiac surgery: invasive versus non-invasive. Poster \& abstract VI researchday NVA, 25th September 2009

- Simons A. P., Reesink K. D., Lancé M. D., vd Nagel T, vd Veen F. H., Weerwind P. W., Maessen J. G., Dynamic filling index-monitoring circulatory filling during minimized extracorporeal bypass, poster $13^{\text {th }}$ annual update on cardiopulmonary bypass, Whistler BC, Canada, March 2008 
- Lancé M.D., Akkermans W.J.M., Theunissen H.M.S., Marcus M.A.E., Accuracy of non-invasive cardiac-output monitoring in cardiac surgery, poster \& abstract 11th International Congress of Cardiothoracic and Vascular Anesthesia, Berlin, Germany, September 2008

- Houben K. W., Lancé M.D., Willigers H.M.M., Scheffers I.J.M., Anesthesie bij barostimulatie implantaties, Abstract O25, NVA 2007, 31

- Reinges M. H. T., Lancé M., Hans F. J., Rhode V., Gilsbach J. M., Hirndruckmonitoring während modifizierter Dilatationstracheotomie nach Griggs, Intensiv Notffallmed 41, suppl 1, 2004, 55-56

- Haltern C., Lancé M., Rossaint R., Unger JK, Does the colloid osmotic pressure gradient in a plasmafilter correlate with sieving profile? poster 48 , ESAO 09/2003

\section{Oral presentations:}

- "Help mijn patiënt bloed", kleine refereeravond anesthesiologie, April 2007

- "Cardiac performance-van preload and afterload", B-cursus, October 2007 en November 2007

- "Hebben we de bloeding onder control?", 20 jaar academiesering anesthesiologie AZM, December 2007

- "Cardiac performance-van preload and afterload", B-cursus, mei 2008 en November 2008

- "Blik op hemodynamiek"\& "ultrasonic flow measurements and alternatives" 11 Februari 2009, 1ste symposium haemodynamische monitoring en ondersteuning IC MUMC+

- "Monitoring van hartdrukken" Cardiologie dag, Limburgse vereniging voor intensive care zorgverlening, 2 Maart 2009

- "Massaal bloedverlies" $1^{\text {ste }}$ blauwe plekken symposium, Maastricht, 14 maart 2009

- Massaal bloedverlies met casuïstieken: hematologie symposium, Maastricht, 16.09.2009

- "Thoracic trauma" landelijk D-cursus onderwijs, 21 April 2010

- "Transport of thrombocyte concentrates by a pneumatic system-facts and fiction" NVA dagen mei 2010 
- "Echocardiography in anaesthesia" NVA dagen mei 2010

- "Peripartaal bloeding-de anesthesiologische kant" NVA dagen mei 2010

- "Oude en nieuwe antistollingsmiddelen-preventie van DVT's en omgang met risiko's" refereeravond orthopedie Maastricht, 7 September 2010

- "Massaal bloedverlies" ROIG, Maastricht 21 Oktober 2010

- Extracorporeal Life Support-case report meeting Maastricht 20.11.2010 chair and day moderator

- "Pro and anticoagulation in cardiac anesthesia" $26^{\text {th }}$ International Winter Symposium UZ Leuven-Belgium, $7^{\text {th }}$ January 2011

- "What's new in dilutional coagulopathy?" Euroanesthesia Amsterdam, $13^{\text {th }}$ June 2011

- "Pre-operatieve screening en bloedingsneiging: hoe, wat en wanneer?", PAOKC/VHL symposium-hemostase naar een nieuwe fase, 6 Oktober 2011, Zwolle

- "Massaal bloedverlies-state of the art", refereeravond Afdeling Anesthesiologie JBZ, Den Bosch, 01.November 2011

- "Cardiac output measurement-state of the art", NVA dagen, Maastricht 10-11. Mei 2012

- "Gerinnungshemmung \& was man dazu wissen muss!" Symposium Leitlinien in der Intensivmedizin, Lünen, 11. Juni 2012

- "The management of critical bleeding in trauma and obstetrics" Workshop Cesena Italy $18^{\text {th }}$ October 2012

- "Levosimendan-a long trip to better understanding" Symposium What else is new- thema dag van de sectie Cardio Anesthesiologie, Den Haag, 10 November 2012

- "ROTEM connectiviteit in het MUMC+", ROTEM super user meeting, 23 November 2012 Mijdrecht

- "Thoracic trauma" landelijk D-cursus onderwijs, 10 en 23 Januari 2013

- "Monitoring coagulation; conventional versus point of care monitoring" Update in Transfusion Medicine, 1 February 2013, Zwolle

- "The NATA European Benchmark study, current state" Update in Transfusion Medicine, 2 February 2013, Zwolle 
- "Point-of care monitoring in modern anesthesia", refereeravond Erasmus MC, 12 March 2013, Rotterdam

\section{Awards:}

- Poster award of the German Cardiothoracic scientific society, November 2011

- Prof. Ritsema van Eck award 2012 